\title{
V. MAGNETOHYDRODYNAMIC EQUILIBRIUM AND STABILITY
}

\author{
S. C. JARDIN (PPPL), M. G. BELL (PPPL), J. L. JOHNSON (PPPL), S. M. KAYE (PPPL), \\ C. KESSEL (PPPL), R. LAHAYE (GA), J. MANICKAM (PPPL), R. PILLSBURY (MIT), \\ N. POMPHREY (PPPL), A. REIMAN (PPPL), R. O. SAYER (ORNL), D. J. STRICKLER (ORNL)
}

In this chapter, we report on the physics studies that have been done to support the design of the poloidal field system in BPX. These studies have been performed in some depth. We briefly summarize the analysis methods used, but concentrate on giving the key results.

\section{V.A. BPX PF SYSTEM OVERVIEW}

The layout of the major components of the BPX poloidal field (PF) system is shown in Fig. 5.1. External to the toroidal field (TF) magnet and assembly are the seven up-down symmetric pairs of primary PF-producing coils. These are called the external PF coils. They are labeled in pairs, with PFIU being the inner (small major radius) PF coil closest to and above the midplane and PF2U-PF7U proceeding clockwise around the machine. Similarly, PF1L is the reflection of PF1U about the midplane, and PF2L-PF7L proceds counterclockwise around the machine to the outside.

The external PF coils have three major functions during a BPX discharge. First, they are the induction coils that provide the flux linkage (or volt-seconds) required to build up and sustain the plasma current by carrying coil currents changing in the opposite direction. Second, they are the shaping coils that produce and control the plasma shape and details of the divertor sweep. Third, they provide control of both the radial and vertical plasma position on time scales greater than about $100 \mathrm{~ms}$. Each of these functions is described in detail in later sections.

In addition to the external PF coils, there are two smaller up-down symmetric pairs of coils located inside the TF magnet, but outside the vacuum vessel. These are called the internal control (IC) coils. The upper coils, IC1U and IC2U, are numbered clockwise, and the lower coils, IC1L and IC2L, are numbered counterclockwise in the same convention as for the external coils.

The IC coils have three major functions. They provide the fast time scale vertical control of the wall stabilized axisymmetric instability as discussed in Sec. V.E. They also provide control of the radial plasma position on the fast time scale, enabling the plasma to recover, for example, from a minor disruption or to have its position adjusted relative to the radio-frequency (RF) antenna, as edge plasma parameters change. In addition, they are used to adjust the poloidal field strength and gradient during the initial plasma breakdown.

The close-fitting metallic vacuum vessel plays a role in the analysis of the PF system because it provides partial stabilization to the vertical axisymmetric instability and because it partially shields the plasma from the fields of time-varying currents in the PF coils. Additional metallic structure associated with the TF coils and their structural supports have similar effects.

We list the vacuum vessel time constants with respect to the penetration of different field patterns in Table 5.1. The first three entries concern

Table 5.1. Vacuum Vessel Time Constants for BPX.

\begin{tabular}{|l|c|c|}
\hline Field Pattern & $L / R(\mathrm{~ms})$ & $R(\mu \Omega)$ \\
\hline Ohmic & 112.5 & 24.3 \\
Symmetric Vertical & 38.1 & NA \\
Antisymmetric Radial & 58.1 & NA \\
Toroidal Field & 32.5 & 15.7 \\
\hline
\end{tabular}

the poloidal field penetration and thus cause vessel currents to develop in the toroidal direction. The ohmic pattern is that corresponding to the field null solution. The symmetric vertical is a uniform magnetic field in the vertical direction, symmetric about the midplane. The antisymmetric radial is the field pattern produced with equal and opposite currents in the two ICl coils. The final entry is the characteristic time for the toroidal field to penetrate. This causes vessel currents to develop in the poloidal direction.

Typical preprogrammed waveforms ${ }^{1}$ of the currents, the energy required to drive them, and the associated flux linkage to the plasma of each of the external PF coils for the standard 11.8-MA updown symmetric double-null divertor 10-s flattop 500-MW discharge are shown in Figs. 5.2, 5.3, and 5.4. We see that the seven coil currents vary in time independently of one another as each of their individual ratios of induction to shaping function change during the shot. PF1 is seen to require 
Jardin et al. MHD EQUILIBRIUM AND STABILITY

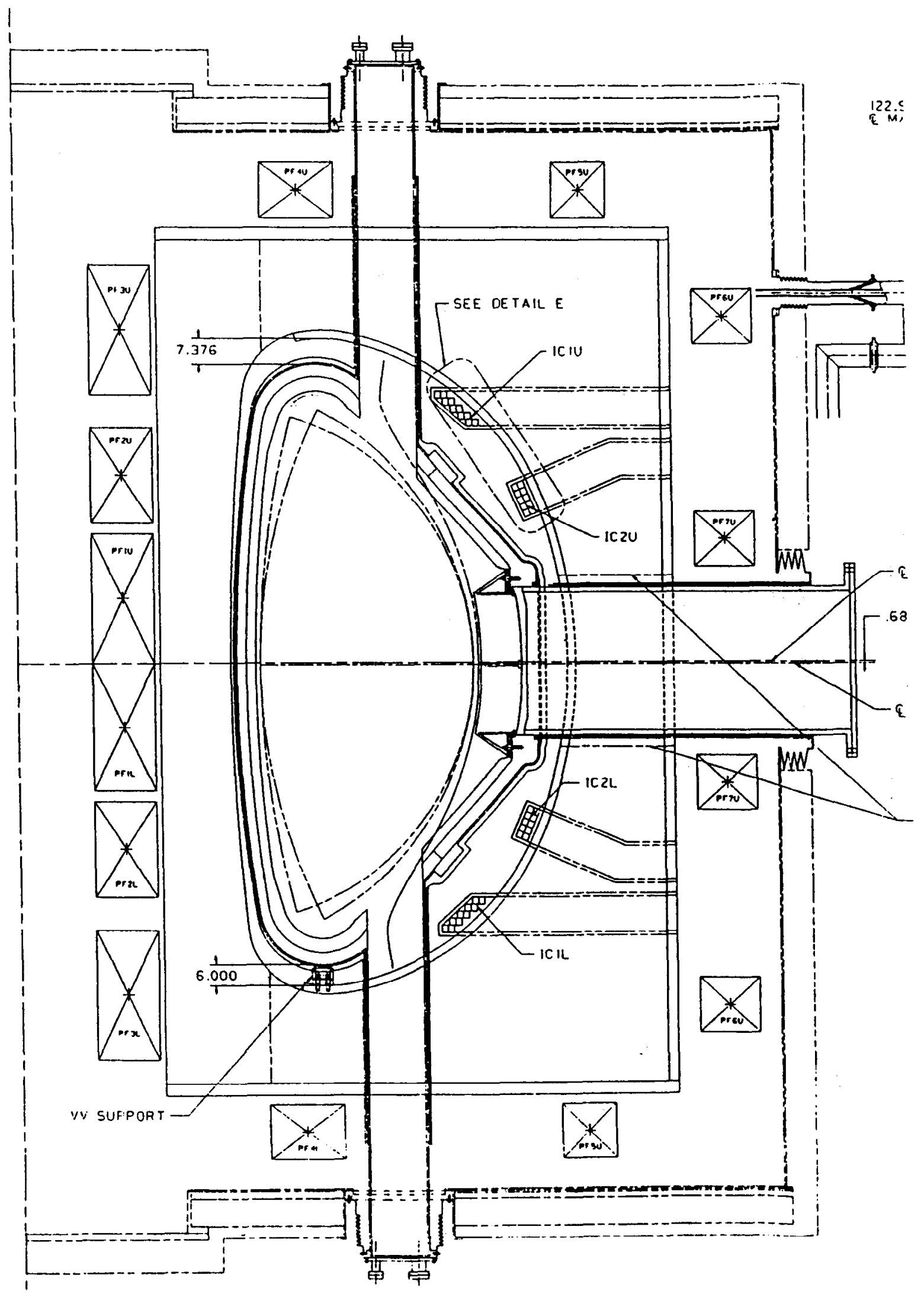

Fig. 5.1. Cross section showing the BPX poloidal field coil system. 


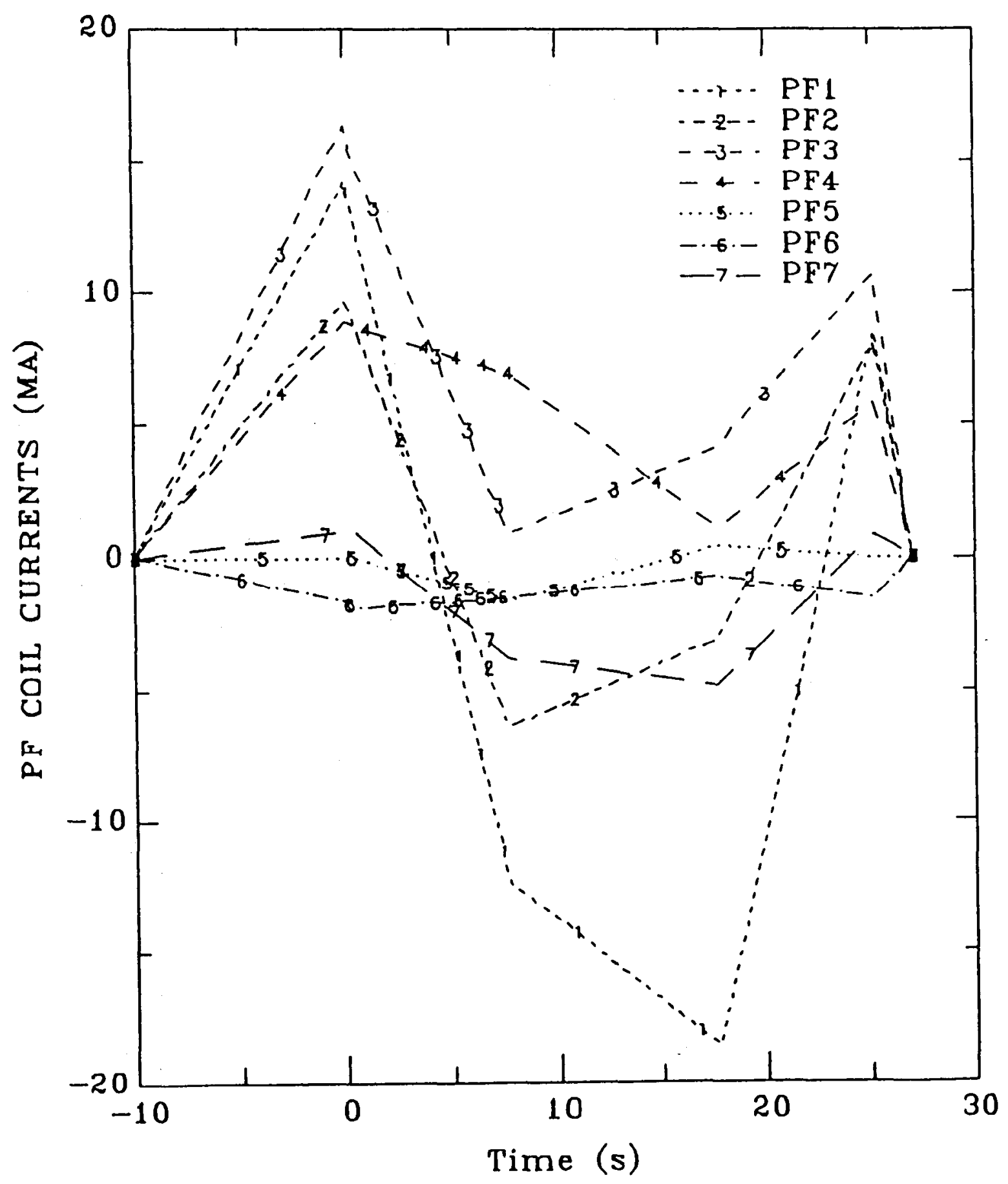

Fig. 5.2. Typical PF coil currents versus time. 
Jardin et al. MHD EQUILIBRIUM AND STABILITY

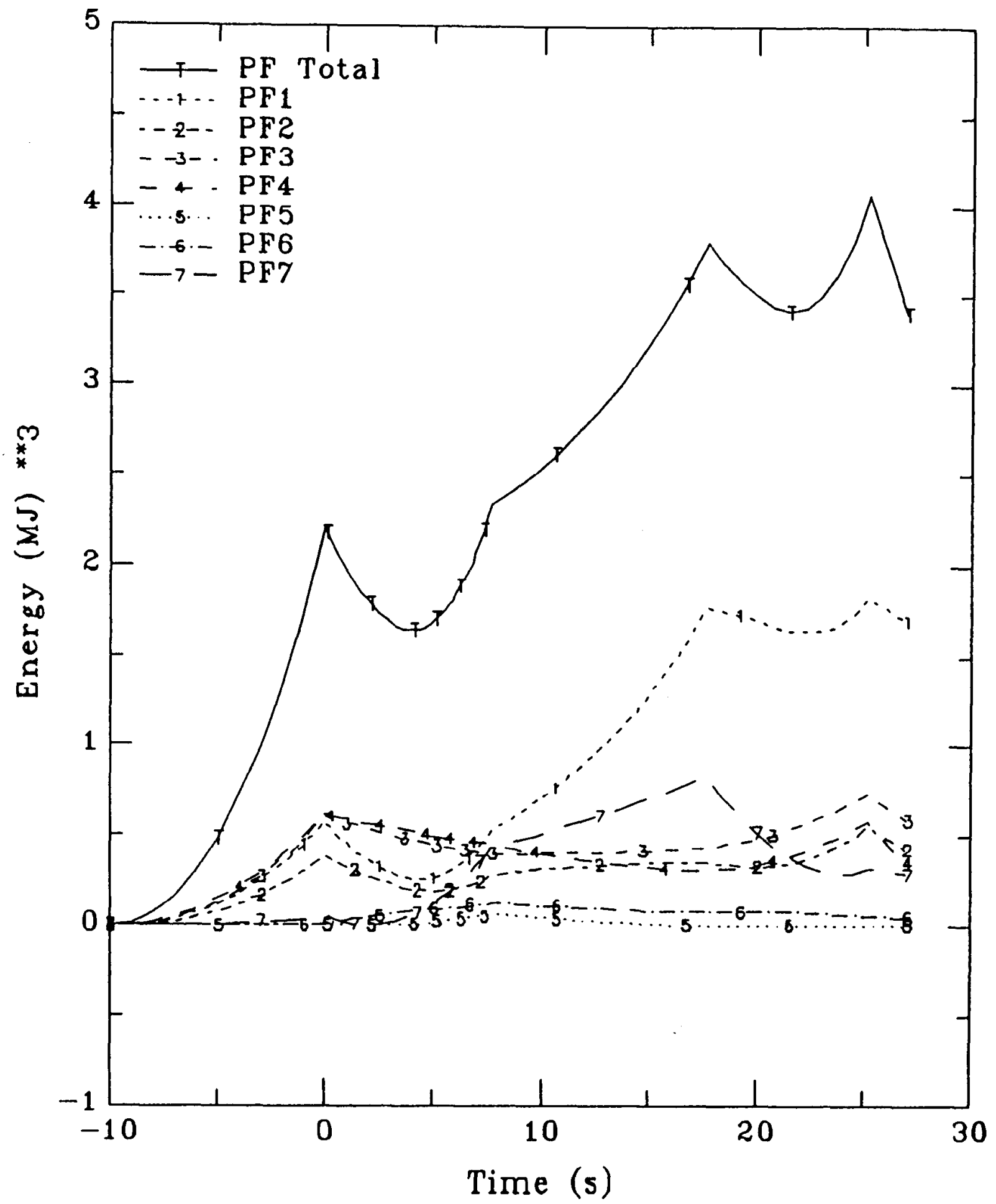

Fig. 5.3. Poloidal field system energy versus time. 

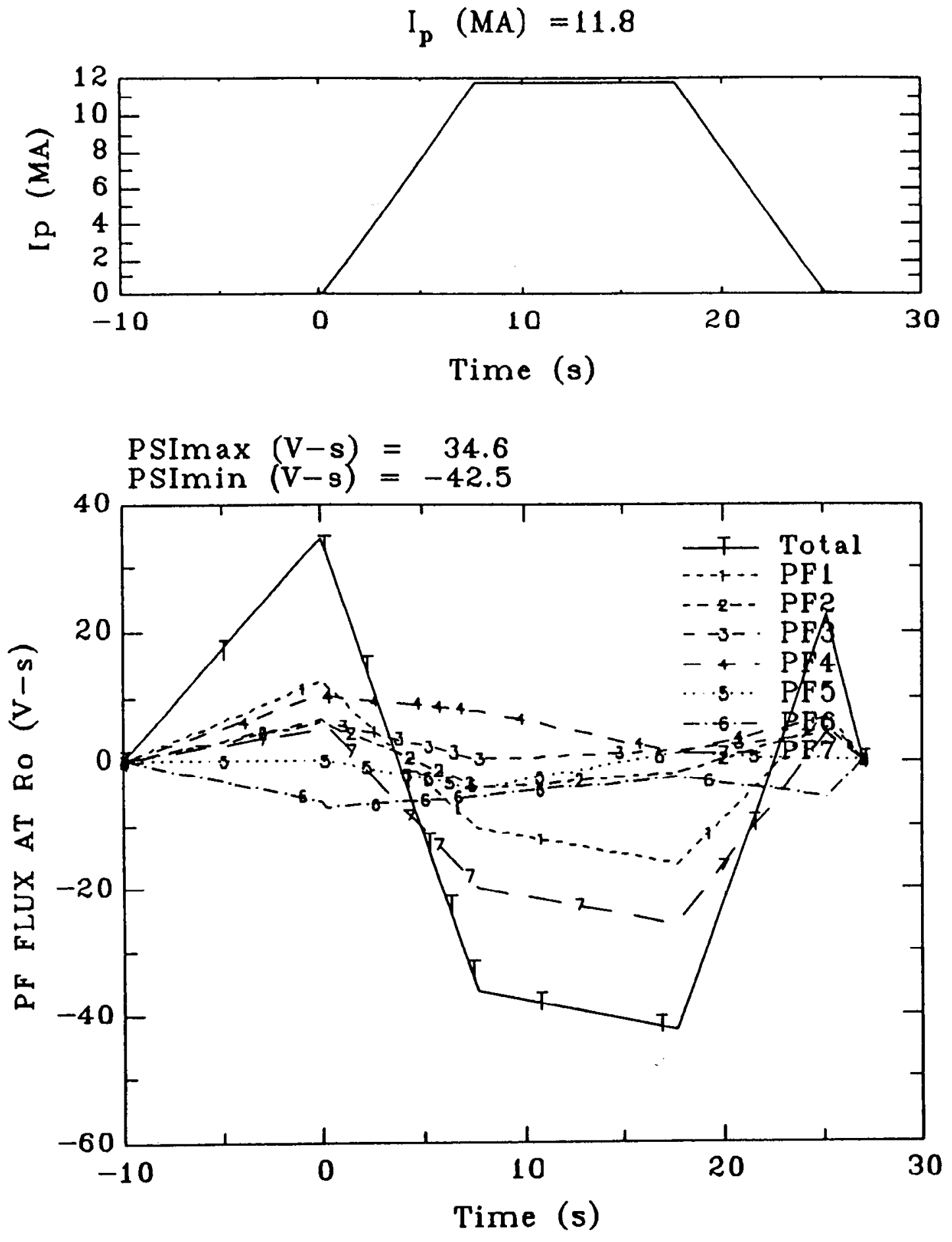

Fig. 5.4. Poloidal field plasma flux linkage versus time. 
the most energy, both because it carries the most current and because it heats up the most during the discharge, causing its resistance, and hence its resistive dissipation, to increase.

It is interesting to note from Fig. 5.4 that PF7 provides the most flux linkage at the plasma center. The efficiency of this outer ring coil in producing flux linkage accounts for the fact that the PF system can provide more flux linkage for high-beta plasmas, where PF7 is called upon to produce a larger vertical field, than it can for low-beta plasmas.

Section V.B describes the magnetohydrodynamic (MHD) equilibrium analysis, examining both the flexibility of the PF system and the sensitivity of the coil current distribution to plasma profiles. Section V.C discusses the requirements for plasma startup. Section V.D describes the automatic shape control designed for BPX. This is broken into two subproblems dealing with boundary detection and error correction. Sections V.E and V.F describe the faster time scale feedback systems that utilize the IC coils to control the plasma vertical and radial position. Section V.G presents several fiducial discharge simulations using the Tokamak Simulation Code ${ }^{2}$ (TSC). This presents a comprehensive simulation model of both the plasma and the PF system components for the entire duration of the discharge.

In Sec. V.H, we present the model and results of disrupting plasma simulations in BPX using the TSC code. This analysis is used to define worst-case disrupting plasma events for use in engineering analysis calculations. Section V.I summarizes the nonaxisymmetric stability calculations that have been done. In Sec. V.J, we describe the basis for the tolerances set for nonaxisymmetric field errors, and in Sec. V.K, we discuss how these translate into tolerances on the construction and installation of the PF coils. Finally, in Sec. V.L, we describe the calculations performed concerning TF ripple in BPX.

In all, we find the design to be good. Assuming each of the PF and control system components operates at its rated capacity, the BPX device as described here should be capable of meeting all relevant requirements set forth in the General Requirements Document (GRD). These include the ability to produce plasma discharges with the required shape and plasma parameters, and the ability to maintain and control these over the time of the discharge. The flux-swing capacity of the PF set should be sufficient to maintain the plasma current for the full discharge time during all the standard operating modes. The thermonuclear burn should be st ably controlled, and the plasma conditions should be well within the stable boundaries in the MHD operating space.

\section{V.B. MHD EQUILIBRIUM}

The role of MHD equilibrium analysis in the design of the BPX is to compute optimal PF coil current distributions, consistent with engineering constraints and the plasma guidelines given in the GRD (Ref. 3) This examination includes

1. the flexibility of the PF system with respect to different operating modes - e.g., double-null (DN) divertor, single-null (SN) divertor, limiter, varying $\kappa$

2. the sensitivity of the coil current distribution to uncertainties in plasma pressure and current distribution and to variations in plasma shape parameters.

The results of this analysis, in the form of coil current distributions, poloidal magnetic field data, and plasma shape control matrices, are used in TSC fiducial discharge simulations, vertical stability calculations, disruption analyses, divertor and limiter design, and in the coil and power system design process.

Calculations are performed with the VEQ (Ref. 4) and control matrix ${ }^{5,6}$ (CM) equilibrium codes. These codes are capable of computing solutions with prescribed plasma current, field on axis, plasma position (major and minor radius), plasma flux linkage (volt-seconds), and various combinations of shape parameters (e.g., $\kappa, \delta, X$-point coordinates, divertor strike-points). Double-null diverted, single-null diverted, and limiter solutions are possible, and constraints on profile parameters (e.g., $\beta_{p}, \ell_{i} / 2$ ) may be imposed. Throughout this chapter we use the standard definitions

$$
\beta_{p}=\frac{4 \int P d V}{\mu_{o} R I_{p}^{2}}
$$

and

$$
\ell_{i}=\frac{2 \int B_{p}^{2} d V}{\mu_{o}^{2} R I_{p}^{2}} .
$$

Except where otherwise indicated, calculations reported here use the GEM-46 (Ref. 7) PF coil set listed in Table 5.2, where $R$ and $Z$ are the coordinates of the upper coil pack current center, and $\Delta R$ and $\Delta Z$ are the coil pack width and height, respectively.

In the following sections, we consider the 500MW DN divertor, the 100-MW DN divertor, limiter-limited configurations, several SN divertor configurations, variations in plasma shape and profile, and the possibilities of forming plasmas with varying elongation and of operating with a reversed sweep direction. 
Table 5.2. Reference PF Coil Set (GEM-46).

\begin{tabular}{|c|c|c|c|c|}
\hline PF Coil & $R(\mathrm{~m})$ & $Z(\mathrm{~m})$ & $\Delta R(\mathrm{~m})$ & $\Delta Z(\mathrm{~m})$ \\
\hline 1 & 0.778 & 0.473 & 0.460 & 0.945 \\
2 & 0.778 & 1.375 & 0.460 & 0.700 \\
3 & 0.778 & 2.438 & 0.460 & 0.945 \\
4 & 2.100 & 3.450 & 0.550 & 0.400 \\
5 & 4.200 & 3.450 & 0.400 & 0.400 \\
6 & 5.200 & 2.530 & 0.450 & 0.400 \\
7 & 5.200 & 0.900 & 0.450 & 0.400 \\
\hline
\end{tabular}

\section{V.B.1. Double-Null Divertor}

The standard BPX plasma is the $P_{\text {fus }}=500 \mathrm{MW}$ DN divertor. The PF configuration of the DN divertor is used in the derivation of PF coil locations, in design of the limiter and divertor plates, and to determine the shape of the ion cyclotron resonance frequency (ICRF) antenna. Figure 5.5 shows equilibrium solutions for beginning of flattop (BOFT) and end of burn (EOB). Plasma profile parameters and flux linkage are fixed at values determined from a preliminary TSC transport simulation and are given in Table 5.3. Equilibria are computed for prescribed $95 \%$ shape parameters and divertor
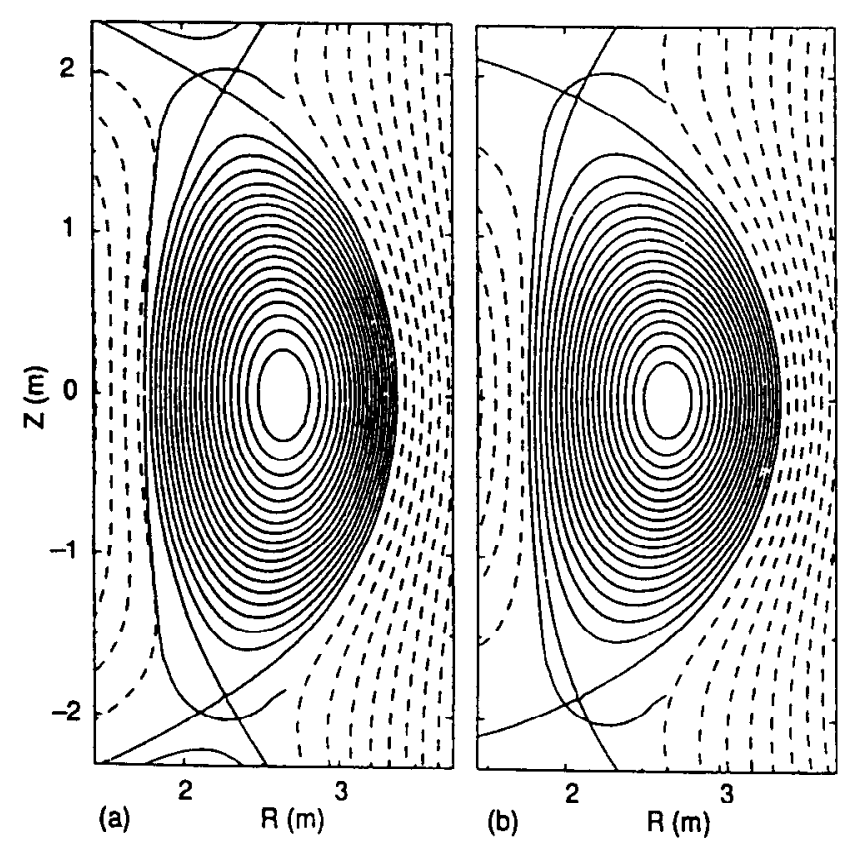

Fig. 5.5. Equilibrium poloidal flux surfaces for the DN diverted plasma at (a) beginning of flattop (BOFT) and (b) end of burn (EOB).
Table 5.3. Fixed Plasma Parameters for the 500MW DN Equilibria.

\begin{tabular}{|l|r|r|}
\hline & BOFT & EOB \\
\hline Major radius (m) & 2.590 & 2.590 \\
Minor radius (m) & 0.794 & 0.794 \\
Elongation (95\%) & 2.100 & 2.000 \\
Triangularity (95\%) & 0.250 & 0.350 \\
Flux linkage (V-s) & 33.000 & 39.200 \\
Plasma current (MA) & -11.800 & -11.800 \\
Ficld on axis (T) & 9.000 & 9.000 \\
Poloidal beta $\left(\beta_{p}\right)$ & 0.210 & 0.385 \\
Internal inductance $\left(\ell_{i} / 2\right)$ & 0.350 & 0.431 \\
\hline
\end{tabular}

strike-points where the separatrices intersect the divertor plates. Elongation at BOFT is $\kappa_{95}=2.1$ in order to achieve the minimum safety factor $q_{95}=$ 3.2. At EOB, elongation is reduced to $\kappa_{95}=2.0$; however, $q_{95}$ exceeds 3.4 as a result of increased triangularity.

Adequate coupling to the ion cyclotron resonance frequency (ICRF) antenna introduces an additional plasma shape requirement: For fixed outer radius on the midplane $R_{o}+a$, the radius $R_{e}$ of the outer plasma edge at an elevation $Z= \pm 0.48$ $m$ should vary by al most $\pm 0.01 \mathrm{~m}$ from a given reference value. For the equilibrium calculations presented here, this reference value is $R_{e}^{r e f}=3.325$ $\mathrm{m}$, based on the DN divertor estimates at BOFT $\left(R_{e}=3.33 \mathrm{~m}\right)$ and $\mathrm{EOB}\left(R_{e}=3.32 \mathrm{~m}\right)$. For different plasma shapes, the distance between the radial edge coordinate at $Z=0.48$ and this reference value will be referred to as $\Delta R_{e}=R_{e}-R_{e}^{\text {ref }}$. Values of $R_{e}$ such that $\left|\Delta R_{e}\right| \leq 1 \mathrm{~cm}$ are considered acceptable, while $1 \mathrm{~cm}<\left|\Delta R_{e}\right| \leq 2 \mathrm{~cm}$ is marginal, and $\left|\Delta R_{e}\right|>2 \mathrm{~cm}$ would require a reconfiguration of the antenna. The geometry and currents for the standard 500-MW DN divertor at BOFT and EOB are given in columns 1 and 3 of Table 5.13.

While most design parameters are based on the $500-\mathrm{MW}$ DN divertor, the 100-MW DN divertor operation imposes the greatest demand on voltseconds. The coil currents for this are listed in Table 5.4 (in addition, see Sec. V.G).

\section{V.B.2. Inner Wall Limiter}

Shape control parameters used in the limiter equilibrium calculations are $R_{o}+a, \kappa$ and $\delta$ at the plasma edge, volt-seconds, and the relative flux dif- 
Table 5.4. 100-MW DN Solutions at EOB for the Reference $\kappa_{95}=2.0$ Elongation

\begin{tabular}{|c|c|}
\hline Plasma & \\
$\kappa_{95}$ & 2.000 \\
$\delta_{95}$ & 0.350 \\
$q_{95}$ & 3.505 \\
$R_{x}(\mathrm{~m})$ & 2.003 \\
$Z_{x}(\mathrm{~m})$ & 1.868 \\
$\Delta R_{e}(\mathrm{~m})$ & 0.000 \\
Coil Currents (MA) & \\
PF1 & 17.400 \\
PF2 & -0.217 \\
PF3 & -3.660 \\
PF4 & -1.980 \\
PF5 & -0.097 \\
PF6 & 0.605 \\
PF7 & 4.855 \\
Flux, Stored Energy & \\
Flux (V-s) & 39.787 \\
$W_{P F}$ (GJ) & 1.299 \\
\hline
\end{tabular}

Ilere, $\beta_{p}=0.2, \ell_{i} / 2=0.458, I_{p}=-11.8 \mathrm{MA}$, $B_{t}=9 \mathrm{~T}$.

ference between the nearby vacuum separatrix flux surface and the limiter flux surface,

$$
d \Psi_{X-L}=\left(\Psi_{x}-\Psi_{L}\right) /\left(\Psi_{L}-\Psi_{a}\right)
$$

where $\Psi_{a}$ is the flux at the magnetic axis. For a configuration to be considered a limiter plasma, the heat flux should be deposited on the limiter surface, not the divertor area. This implies that the flux difference between the limiter contact point and the $\mathrm{X}$-point be greater than the scrape-off flux, or $\Delta \Psi_{s} \leq d \Psi_{X-L}\left(\Psi_{L}-\Psi_{a}\right)$, where $\Delta \Psi_{s}$ is the scrape-off flux. Increasing $d \Psi_{X-L}$ moves the vacuum separatrix flux surface further from the plasma edge. It is required that the flux difference (corresponding to $\Delta R=2 \mathrm{~cm}$ at the outboard edge),

$$
\Delta \Psi_{2 c m}=(0.02) \partial \Psi / \partial R,
$$

measured at $R=R_{o}+a, Z=0$, places an upper bound on $\Delta \Psi_{s}$ and that the flux surface $\Psi\left(R_{o}+a, 0\right)+\Delta \Psi_{2 c m}$ should intersect the outboard limiters or divertor plates in at most one point. A limiter solution at BOFT with $d \Psi_{X-L}=$ 0.06 is presented in Fig. 5.6, and fixed parameters are listed in Table 5.5.

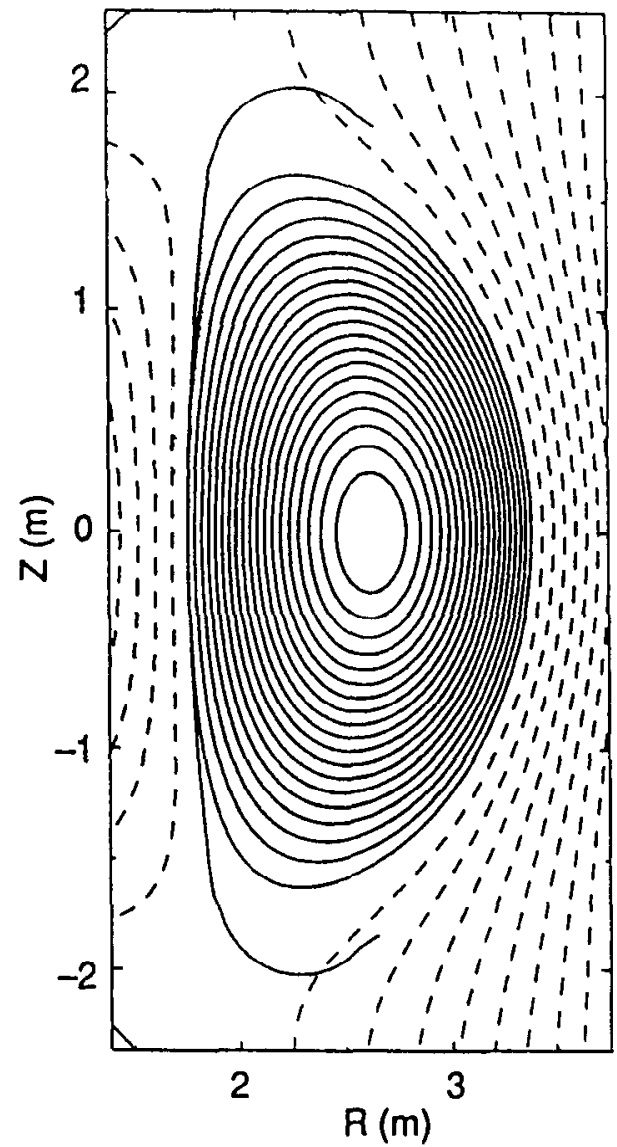

Fig. 5.6. Limiter equilibrium at BOFT.

\section{V.B.3. Single-Null Divertor}

For SN divertor equilibria, important plasma shape considerations include

1. the position of the active X-point (assumed here to be the upper null), in order to maintain adequate distances between the X-point and divertor strike-points, together with a sufficient sweep distance

2. the flux difference between the active and nonactive $\mathrm{X}$-points

3. the flux difference between the active X-point and the opposite divertor plate

4. maximizing the plasma volume within the vacuum vessel, subject to limitations (e.g., vertical stability, scrape-off requirements) on the elongation.

Assuming the same upper limit for the scrapeoff flux as for the limiter plasma, we want solutions such that

1. $0 \leq \Delta \Psi_{s} \leq \Delta \Psi_{2 \mathrm{~cm}}$

2. $\Delta \Psi_{s} \leq d \Psi_{x}\left(\Psi_{x}-\Psi_{a}\right)$ 
Table 5.5. Parameters for the 500-MW Limiter Equilibrium

\begin{tabular}{|l|r|r|}
\hline & BOFT & EOB \\
\hline Plasma & & \\
$R_{o}(\mathrm{~m})$ & 2.572 & 2.572 \\
$a(\mathrm{~m})$ & 0.814 & 0.814 \\
$I_{p}(\mathrm{MA})$ & -11.770 & -11.770 \\
$B_{t}(\mathrm{~T})$ & 9.063 & 9.063 \\
$\beta_{p}$ & 0.200 & 0.430 \\
$\ell_{i} / 2$ & 0.380 & 0.400 \\
$q_{\text {axis }}$ & 1.025 & 0.894 \\
$q_{95}$ & 3.064 & 3.121 \\
$q_{\text {edge }}$ & 3.784 & 3.994 \\
$d \Psi_{X-L}$ & 0.060 & 0.050 \\
$\kappa_{\text {edge }}$ & 2.000 & 2.000 \\
$\delta_{\text {edge }}$ & 0.350 & 0.350 \\
$\Delta R_{e}$ & 0.002 & 0.002 \\
PF Currents (MA) & & \\
PF1 & 14.013 & 14.000 \\
PF2 & 0.718 & 1.605 \\
PF3 & -1.132 & -0.496 \\
PF4 & -3.310 & -2.989 \\
PF5 & 1.013 & 0.515 \\
PF6 & 1.467 & 1.216 \\
PF7 & 3.642 & 4.319 \\
\hline
\end{tabular}

3. $\Psi_{s}$ intersects the lower divertor plate in at most one point

\section{4. $\kappa_{95} \leq 2$.}

Here $d \Psi_{x}$ is the control parameter used in the $\mathrm{CM}$ equilibrium code, measuring the relative difference between the upper X-point flux $\Psi_{X U}$ and the lower X-point flux $\Psi_{X L}$, i.e.,

$$
\begin{gathered}
d \Psi_{x}=\left(\Psi_{X L}-\Psi_{X U}\right) /\left(\Psi_{x}-\Psi_{a}\right), \\
\Psi_{x}=\min \left(\Psi_{X L}, \Psi_{X U}\right) .
\end{gathered}
$$

Single-null solutions for BOFT and EOB are displayed in Fig. 5.7, and the plasma parameters listed in Table 5.6. Of particular interest is the fact that for these solutions the radial coordinates of the outboard edge at $\pm 0.48 \mathrm{~m}$ both lie within the acceptable interval for ICRF coupling. The $95 \%$ elongation at EOB is slightly less than 2 in order to satisfy the flux inequalities (1) to (3) above.
Table 5.6. Plasma Parameters for the 500-MW SN

\begin{tabular}{|c|c|c|}
\hline & BOFT & EOB \\
\hline \multicolumn{3}{|l|}{ Plasma } \\
\hline$R_{o}(\mathrm{~m})$ & 2.590 & $2.590^{*}$ \\
\hline$a(\mathrm{~m})$ & 0.795 & $0.795^{*}$ \\
\hline$I_{p}(\mathrm{MA})$ & -11.800 & $-11.800^{*}$ \\
\hline$B_{t}(\mathrm{~T})$ & 9.000 & $9.000^{*}$ \\
\hline \multicolumn{3}{|l|}{ Profile Parameters } \\
\hline$\ell_{i} / 2$ & 0.353 & 0.436 \\
\hline$\beta_{p}$ & 0.211 & 0.388 \\
\hline \multicolumn{3}{|l|}{ X-points } \\
\hline$R_{x-u}(\mathrm{~m})$ & 2.268 & $2.083^{*}$ \\
\hline$Z_{x-u}(\mathrm{~m})$ & 1.891 & $1.866^{*}$ \\
\hline$R_{x-L}(\mathrm{~m})$ & 2.050 & $2.083^{*}$ \\
\hline$Z_{x-L}(\mathrm{~m})$ & -1.900 & $-1.972^{*}$ \\
\hline$d \Psi_{x}$ (normalized) & 0.045 & $0.043^{*}$ \\
\hline \multicolumn{3}{|l|}{ Plasma Shape } \\
\hline$\kappa_{95}$ & 2.039 & 1.959 \\
\hline$\delta_{U, 95}$ & 0.259 & 0.350 \\
\hline$\delta_{L, 95}$ & 0.274 & 0.251 \\
\hline$\Delta R_{e-U}(\mathrm{~m})$ & 0.004 & -0.009 \\
\hline$\Delta R_{e-L}(\mathrm{~m})$ & 0.008 & 0.007 \\
\hline \multicolumn{3}{|l|}{ PF Currents (MA) } \\
\hline PF1 & 11.424 & 15.785 \\
\hline PF2 & 3.972 & -0.126 \\
\hline PF3-U & -2.022 & -1.877 \\
\hline PF3-L & -9.776 & -0.163 \\
\hline PF4-U & -7.216 & -3.720 \\
\hline PF4-L & -3.117 & -2.815 \\
\hline PF5 & 1.913 & -0.085 \\
\hline PF6-U & 1.168 & 1.552 \\
\hline PF6-L & -0.062 & 0.637 \\
\hline PF7 & 3.998 & 4.775 \\
\hline \multicolumn{3}{|l|}{ Stored Energy, Flux } \\
\hline$W_{P F}(\mathrm{GJ})$ & 1.139 & 1.289 \\
\hline V-s & 33.000 & $39.200^{*}$ \\
\hline
\end{tabular}
Equilibria

*Indicates the parameter is fixed in the equilibrium calculation.

\section{V.B.4. Variation with Plasma Profile}

For fixed plasma position, shape, and flux linkage, the variation in the coil current distribution with poloidal beta is almost linear (Table 5.7). Here the current in the outboard equilibrium field coil PF7 incrcases with $\beta_{p}$ to account for the increase in the required vertical field, while the remaining currents are adjusted to maintain the fixed 
Table 5.7. PF Coil Current Variation with Poloidal Beta for the $R=2.59 \mathrm{~m}$, DN Divertor

\begin{tabular}{|l|r|r|r|r|r|}
\hline Plasma & 0.036 & 0.108 & 0.179 & 0.250 & 0.321 \\
$\beta_{p}$ & 0.187 & 0.560 & 0.931 & 1.300 & 1.667 \\
$\beta(\%)$ & 1.154 & 1.142 & 1.131 & 1.119 & 1.105 \\
$q_{\text {aris }}$ & 3.248 & 3.251 & 3.253 & 3.257 & 3.262 \\
$q_{95}$ & 0.007 & 0.007 & 0.007 & 0.007 & 0.007 \\
$\Delta R_{e}(\mathrm{~m})$ & & & & & \\
Coil Currents (MA) & & & & & \\
PF1 & 11.349 & 11.055 & 10.693 & 10.387 & 10.088 \\
PF2 & 5.921 & 5.786 & 5.786 & 5.692 & 5.594 \\
PF3 & -4.242 & -4.252 & -4.188 & -4.178 & -4.172 \\
PF4 & -6.674 & -6.648 & -6.661 & -6.648 & -6.636 \\
PF5 & 2.069 & 1.984 & 1.876 & 1.788 & 1.704 \\
PF6 & 1.091 & 1.041 & 0.993 & 0.946 & 0.900 \\
PF7 & 3.551 & 3.690 & 3.838 & 3.976 & 4.110 \\
Stored Energy & & & & & \\
WPF (GJ) & 1.179 & 1.181 & 1.187 & 1.194 & 1.201 \\
\hline
\end{tabular}

Fixed parameters include $\kappa_{95}=2.10, \delta_{95}=0.25, \ell_{i} / 2=0.35$, and flux $=33.0 \mathrm{~V} \cdot \mathrm{s}$.
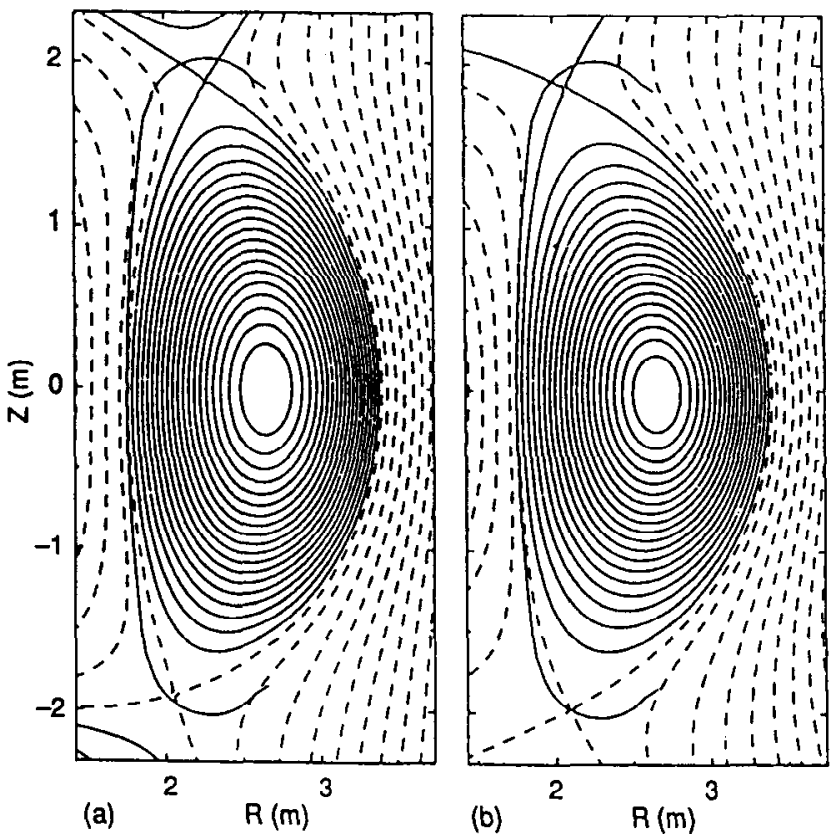

Fig. 5.7. Single-null divertor equilibria at (a) BOFT and (b) EOB.

volt-second and shape conditions.

The coil current variation with the plasma internal inductance $\ell_{i} / 2$ is stronger, as shown in Ta- ble 5.8. Very broad and very peaked profiles both alter the PF shaping field current distribution. The stored energy is seen to have a distinct minimum in the interval $0.30 \leq \ell_{i} / 2 \leq 0.45$ where we expect the normal operating regime of BPX to lie.

\section{V.B.5. Dependence on Changes in Plasma Current Profile not Described by $\ell_{i}$}

The plasma current density profile $J_{\phi}$ is characterized to first order by the internal inductance and poloidal beta. The sensitivity of the coil current distribution to these parameters was discussed in the preceding section. Here we examine the dependence on higher order features in $J_{\phi}$ by constraining $\beta_{p}$ and $\ell_{i} / 2$, and computing equilibria with different current profiles by varying the safety factor at the magnetic axis, $q_{\text {axis }}$. Solutions are presented in Table 5.9, where further constraints include fixed plasma shape, X-point coordinates, volt-seconds, and pressure profile shape.

The plasma toroidal current density profile is represented as

$$
J_{\phi}=R d P / d x+1 /\left(2 \mu_{o} R\right) d F^{2} / d x,
$$

where $x$ is the normalized poloidal flux, $R$ is the major radius, and

$$
d F^{2} / d x=2 \mu_{o}\left(1 / \beta_{J}-1\right) R_{0}^{2}\left(a_{1} \phi_{1}+a_{2} \phi_{2}\right),
$$


Table 5.8. Poloidal Field Coil Current Variation with Internal Inductance

\begin{tabular}{|l|r|r|r|r|r|}
\hline Plasma & & & & & \\
$\ell_{i} / 2$ & 0.263 & 0.307 & 0.368 & 0.429 & 0.564 \\
$\beta_{p}$ & 0.210 & 0.210 & 0.210 & 0.210 & 0.210 \\
$q_{\text {axis }}$ & 1.756 & 1.403 & 1.026 & 0.762 & 0.438 \\
$q_{95}$ & 3.300 & 3.305 & 3.227 & 3.246 & 3.063 \\
$\Delta R_{e}(\mathrm{~m})$ & 0.004 & 0.007 & 0.007 & 0.006 & 0.000 \\
Coil Currents (MA) & & & & & \\
PF1 & 8.400 & 9.592 & 10.917 & 11.980 & 13.527 \\
PF2 & 9.720 & 7.546 & 5.085 & 3.055 & -0.166 \\
PF3 & -2.153 & -3.278 & -4.551 & -5.607 & -7.240 \\
PF4 & -9.436 & -7.914 & -6.275 & -4.968 & -2.993 \\
PF5 & 3.622 & 2.653 & 1.553 & 0.638 & -0.817 \\
PF6 & 3.079 & 1.933 & 0.651 & -0.399 & -2.048 \\
PF7 & 1.925 & 2.995 & 4.221 & 5.247 & 6.907 \\
Stored Energy, Flux & & & & & \\
$W_{P F}$ (GJ) & 1.599 & 1.312 & 1.180 & 1.222 & 1.567 \\
V-s & 33.000 & 33.000 & 33.000 & 33.000 & 33.000 \\
\hline
\end{tabular}

These equilibria assume fixed X-point, where the X-point is taken from a $\kappa_{95}=2.10, \delta_{95}=0.25$, reference BOFT case with $\beta_{p}=0.21$, and $\ell_{i} / 2=0.35$. 
Jardin et al. MHD EQUILIBRIUM AND STABILITY

Table 5.9. Dependence of PF Coil Currents on Higher Order Changes in the Plasma Current Profile

\begin{tabular}{|l|r|r|r|r|}
\hline Equilibrium & \multicolumn{1}{c|}{$\mathrm{A}$} & \multicolumn{1}{c|}{$\mathrm{B}$} & \multicolumn{1}{c|}{$\mathrm{C}$} & \multicolumn{1}{c|}{$\mathrm{D}$} \\
\hline Plasma & & & & \\
$a_{1}$ & 1.000 & 1.000 & 1.000 & 1.000 \\
$\alpha_{1}$ & -0.140 & -1.024 & -2.993 & -4.060 \\
$a_{2}$ & -0.500 & -0.000 & 1.000 & 1.500 \\
$\alpha_{2}$ & 2.000 & 2.000 & 2.000 & 2.000 \\
$\beta_{j}$ & 0.183 & 0.294 & 0.430 & 0.478 \\
$q_{\text {axis }}$ & 1.208 & 1.123 & 1.024 & 0.992 \\
$q_{95}$ & 3.255 & 3.244 & 3.235 & 3.233 \\
Strike-Points (m) & & & & \\
$R_{\text {isp }}$ & 2.106 & 2.105 & 2.106 & 2.106 \\
$Z_{\text {isp }}$ & 1.984 & 1.984 & 1.984 & 1.984 \\
$R_{\text {osp }}$ & 2.346 & 2.346 & 2.347 & 2.347 \\
$Z_{\text {osp }}$ & 2.018 & 2.018 & 2.018 & 2.018 \\
Coil Currents (MA) & & & & \\
PF1 & 10.603 & 10.582 & 10.546 & 10.515 \\
PF2 & 5.692 & 5.723 & 5.769 & 5.817 \\
PF3 & -4.230 & -4.220 & -4.193 & -4.169 \\
PF4 & -6.645 & -6.685 & -6.727 & -6.768 \\
PF5 & 1.819 & 1.835 & 1.842 & 1.860 \\
PF6 & 0.957 & 0.978 & 0.998 & 1.024 \\
PF7 & 3.918 & 3.9054 & 3.894 & 3.875 \\
Stored Energy & & & & \\
$W_{P F}$ (GJ) & 1.191 & 1.194 & 1.198 & 1.201 \\
\hline
\end{tabular}



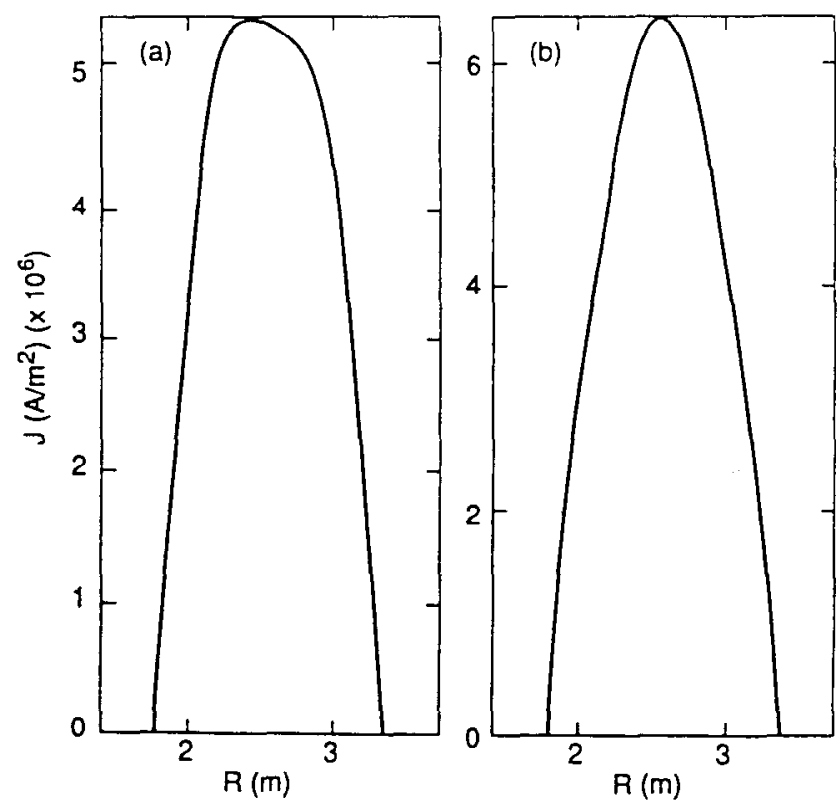

Fig. 5.8. Plasma current density profiles for solutions $\mathrm{A}$ and $\mathrm{C}$ of Table 5.9.

$$
\begin{aligned}
\phi_{i}(x)= & {\left[\exp \left(-\alpha_{i}\right)-\exp \left(-\alpha_{i} x\right)\right] } \\
& /\left[1-\exp \left(-\alpha_{i}\right)\right] i=1,2 .
\end{aligned}
$$

For the present study, the parameters $a_{1}=1.0$ and $\alpha_{2}=2.0$ are held constant, $a_{2}$ is varied, and values of $\beta_{J}$ and $\alpha_{1}$ are found that give $\beta_{p}=0.21$ and $\ell_{i} / 2=0.35$ (BOFT values). In Fig. 5.8, we illustrate two of the midplane current profiles corresponding to columns $A$ and $C$ of Table 5.9. These two equilibria have different values of $q_{\text {axis }}$ (1.2 and 1.0) but the same value of $\ell_{i} / 2$.

The results, summarized in Table 5.9, show that for a $20 \%$ change in $q_{a x i s}$, the coil current distribution remains nearly constant, and the stored energy changes by less than $1 \%$. Also, the strike-point coordinates, where the separatrix surface intersects the divertor plate, remains fixed to within $1 \mathrm{~mm}$. We conclude that the dependence of the coil currents and divertor strike-point locations on $J_{\phi}$ are sufficiently characterized solely by the global parameters $\beta_{p}$ and $\ell_{i} / 2$.

\section{V.B.6. Additional PF System Capabilities}

To further illustrate the range of equilibrium solutions obtainable with the BPX PF coil system, we examine in this section (a) solutions with increased beta, (b) variations with plasma shape, (c) reversed divertor sweep, (d) longer X-point to strike-point distance, and (e) a sequence of equilibria with reduced $I_{p}$, meaning larger $q_{95}$ and $\ell_{i}$. These studies are motivated by the desire to maximize the flexibility of the BPX device. In sev- eral cases, we find that modest compromises in the usual physics requirements, such as shape constraints, are required. The engineering implications of these configurations, which involve coil stresses and temperatures, and power supply demands have not been fully analyzed. Experience suggests that in some cases, if necessary, it may be possible to redistribute $\mathrm{PF}$ currents with only minor effects on shape in order to satisfy engineering constraints.

Table 5.10 presents coil current distributions for

Table 5.10. Double-null, Diverted EOB Equilibria of Fixed Shape, Internal Inductance, and Increasing Beta

\begin{tabular}{|c|r|r|r|}
\hline Plasma & & & \\
$\beta \%$ & 2.000 & 3.500 & 5.000 \\
$\beta_{\text {pol }}$ & 0.379 & 0.665 & 0.953 \\
$q_{95}$ & 3.448 & 3.501 & 3.554 \\
$R_{a}(\mathrm{~m})$ & 2.661 & 2.690 & 2.720 \\
$R_{x}(\mathrm{~m})$ & 2.084 & 2.076 & 2.066 \\
$Z_{x}(\mathrm{~m})$ & 1.866 & 1.868 & 2.869 \\
$\Delta R_{e}(\mathrm{~m})$ & -0.003 & -0.003 & -0.003 \\
Coil Currents (MA) & & & \\
PF1 & 16.000 & 16.000 & 16.000 \\
PF2 & -0.168 & 1.148 & 2.333 \\
PF3 & -1.604 & -0.392 & 0.770 \\
PF4 & -4.006 & -3.294 & -2.593 \\
PF5 & 0.323 & -0.085 & -0.501 \\
PF6 & 1.609 & 1.296 & 0.975 \\
PF7 & 4.383 & 5.030 & 5.678 \\
Stored Energy, Flux & & & \\
$W_{P F}$ (GJ) & 1.308 & 1.443 & 1.614 \\
V-s & 39.159 & 42.504 & 45.679 \\
\hline
\end{tabular}

Plasma shape is prescribed by the parameters $\kappa_{95}=2.0 ; \delta_{95}=0.35$, and $\ell_{i} / 2=0.432$.

a sequence of DN divertor EOB solutions with volume-averaged beta increased to the Troyon limit $\beta=3 I_{p} / a B_{t}=5.0 \%$. In this sequence of fixed $95 \%$ shape solutions, the current in PFl is held constant at $I_{1}=16 \mathrm{MA}$, and the flux linkage is unconstrained. Internal inductance is prescribed to be $\ell_{i} / 2=0.432$. The volt-seconds and stored energy rise with increased current in PF7, but only nominal changes in current are required in the remaining coils, PF2 to PF6. Constraining the flux would actually reduce the PF1 and PF7 currents some- 
Jardin et al. MHD EQUILIBRIUM AND STABILITY
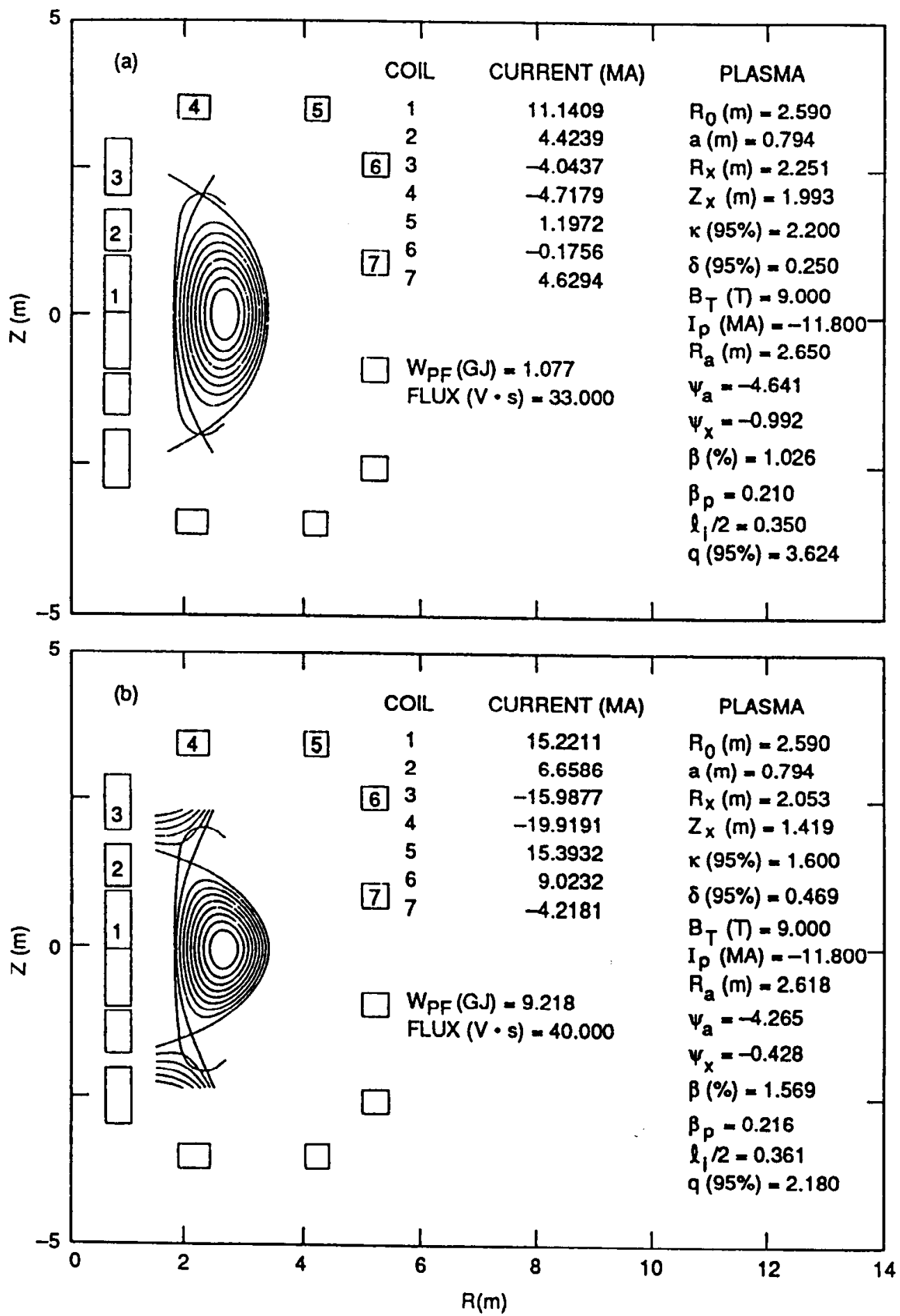

Fig. 5.9. Diverted equilibria at BOFT with elongation (a) $\kappa_{95}=2.2$ and (b) $\kappa_{95}=1.6$. 

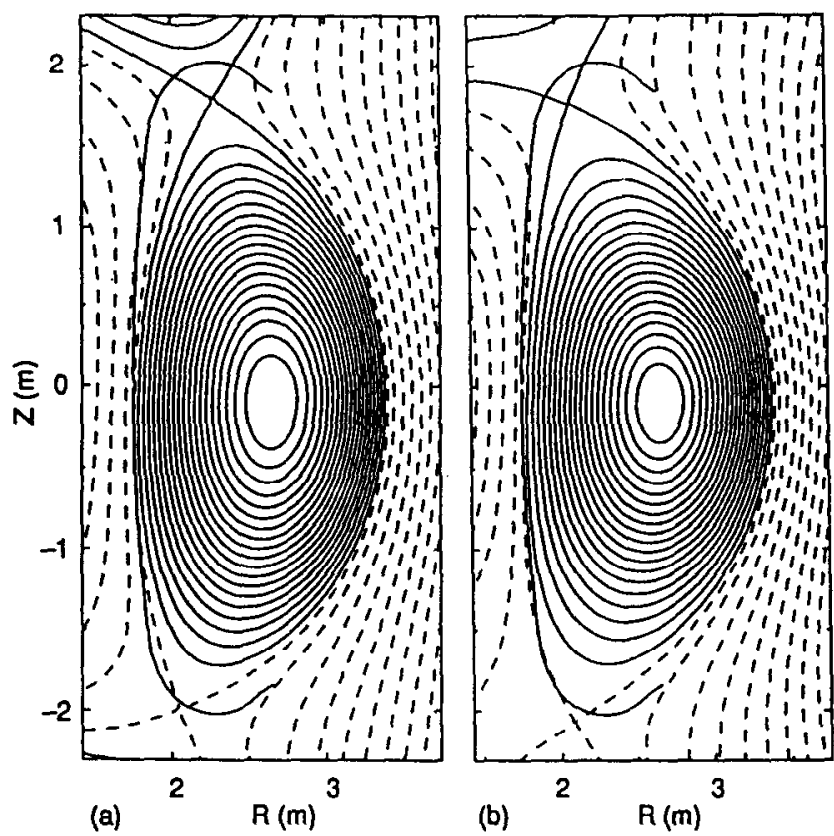

Fig. 5.10. Single-null long divertor mode (a) BOFT and (b) EOB.

what, as shown in Sec. V.B.4. However, divertor heat loads would exceed the power handling capability of the present design, and it is not yet known whether the TF overturning moments would exceed design allowables.

Figure 5.9 shows diverted BOFT solutions for $\kappa_{95}=2.2(\mathrm{X}$-point on the divertor plate such that $\kappa_{x}=2.5$ ) and $\kappa_{95}=1.6$. Higher elongation tends to reduce coil stored energy as the $\mathrm{X}$-point approaches a "natural" position with respect to the PF coil set. Reduced elongation results in substantial increases in coil current magnitude and power supply requirements. Variations in plasma elongation also increase the distance between the plasma edge and the ICRF antenna. While the ability to run with reduced elongation in BPX may be limited, operation with the X-point on the divertor plate is within the capabilities of the PF system. Moreover, the vertical stability of such plasmas appears to be satisfactory, as is discussed in Sec. V.E. This may provide a way to reduce the peak divertor heat fluxes by maximizing the flux expansion at the divertor plate, depending on the erosion rates.

Poloidal field coil current distributions are given in Tables 5.11 and 5.12 for variations in plasma elongation and triangularity, respectively. Variations in triangularity are possible, but lowelongation $\left(\kappa_{95} \leq 1.8\right)$, full-current, DN divertor plasmas impose excessive demands on power supplies and generators. This is a reflection of the
Table 5.11. Double-Null Divertor Variation of Coil Currents with Plasma Elongation at BOFT

\begin{tabular}{|c|r|r|r|r|}
\hline Plasma & 1.800 & 2.000 & 2.096 & 2.200 \\
$\kappa_{95}$ & 1.010 & 1.099 & 1.123 & 1.147 \\
$q_{\text {aris }}$ & 2.324 & 2.918 & 3.243 & 3.635 \\
$q_{95}$ & -0.019 & -0.001 & 0.007 & 0.014 \\
$\Delta R_{e}(\mathrm{~m})$ & & & & \\
Coil & & & & \\
Currents & & & & \\
(MA) & & & & \\
PF1 & 6.589 & 9.871 & 10.545 & 11.144 \\
PF2 & 17.357 & 7.357 & 5.779 & 4.396 \\
PF3 & -13.747 & -4.091 & -4.190 & 4.009 \\
PF4 & -18.023 & -9.579 & -6.729 & -4.675 \\
PF5 & 11.331 & 2.915 & 1.861 & 1.198 \\
PF6 & 0.893 & 2.476 & 1.009 & -0.206 \\
PF7 & 1.721 & 2.892 & 3.877 & 4.641 \\
Stored & & & & \\
Energy & & & & \\
$W_{P F}$ (GJ) & 4.579 & 1.573 & 1.197 & 1.075 \\
\hline
\end{tabular}

Fixed parameters include $\delta_{95 \%}=0.25, \beta_{p}=0.21$, $\ell_{i} / 2=0.35$, and flux $=33.0 \mathrm{~V} \cdot \mathrm{s}$.

well-known fact that higher order multipole fields are needed to produce diverted plasmas with elongations much less than $\kappa_{95}=\mathbf{2 . 0}$.

The capability to vary the divertor sweep pattern, including the possibility of multiple sweeps, is of interest from the standpoint of power handling flexibility. This motivates an investigation of a "reverse-sweep" (R-S) sequence, in which the sweep is from high to low triangularity instead of low to high. The same $\beta_{p}, \ell_{i} / 2$, and volt-seconds are assumed as for the standard BOFT and EOB equilibria. Table 5.13 compares the R-S BOFT and EOB equilibria with those for the standard $500-\mathrm{MW}$ DN discharge. A triangularity of 0.45 at BOFT is needed in order to obtain an adequate outer strike-point sweep distance and $q_{95}>3.2$. The reverse sweep significantly increases the outer $\mathrm{X}$-point to strike-point distance at EOB; however, the inner strike-point distance is reduced at BOFT. Shaping currents in coils PF3 and PF5 are larger at BOFT for the R-S case. This is within the capabilities of the PF3 coils and power supplies, but exceeds the maximum demands on PF5 imposed by the reference discharges. Further engineering 
Table 5.12. Poloidal Field Coil Current

\begin{tabular}{|c|r|r|r|r|}
\hline Plasma & & & & \\
$\delta_{95}$ & 0.050 & 0.150 & 0.250 & 0.350 \\
$q_{\text {axis }}$ & 1.127 & 1.128 & 1.125 & 1.122 \\
$q_{95}$ & 2.976 & 3.093 & 3.254 & 3.468 \\
$\Delta R_{e}(\mathrm{~m})$ & 0.016 & 0.012 & 0.007 & 0.004 \\
Coil & & & & \\
Currents & & & & \\
(MA) & & & & \\
PF1 & 7.073 & 8.849 & 10.558 & 12.183 \\
PF2 & 11.917 & 8.848 & 5.746 & 2.667 \\
PF3 & -0.538 & -2.380 & -4.183 & -6.021 \\
PF4 & -7.115 & -7.107 & -6.655 & -5.634 \\
PF5 & 0.173 & 0.998 & 1.837 & 2.571 \\
PF6 & -0.016 & 0.678 & 0.973 & 0.875 \\
PF7 & 5.110 & 4.413 & 3.899 & 3.595 \\
Stored & & & & \\
Energy & & & & \\
$W_{P F}$ (GJ) & 1.363 & 1.252 & 1.190 & 1.147 \\
\hline
\end{tabular}

Variation with Plasma Triangularity. Fixed parameters include $\kappa_{95}=2.10, \beta_{p}=0.21$, $\ell_{i} / 2=0.35$, and flux $=33 \mathrm{~V} \cdot \mathrm{s}$.

analysis of this sequence is required.

In Table 5.14, single-null "long divertor mode" equilibria with X-points displaced by $d Z_{x}=-10$ $\mathrm{cm}$ are compared to the "standard" SN solutions at BOFT and EOB. These are illustrated in Figs. $5.10 \mathrm{a}$ (BOFT) and $5.10 \mathrm{~b}$ (EOB). This result is characterized by an increased distance between the active (upper) $\mathrm{X}$-point and the divertor strikepoints. Moving the X-points vertically, however, tends to move the plasma edge at $Z= \pm 0.48 \mathrm{~m}$ $\left(\Delta R_{e, u}\right.$ and $\Delta R_{e, \ell}$ in Table 5.14) away from the standard reference points for ICRF coupling. If Xpoints are moved radially to compensate for this, it tends to decrease the scrape-off flux on the inboard side (between the plasma edge and limiter surface) to less than the value corresponding to $2 \mathrm{~cm}$ on the outboard midplane. Changes in the coil current distribution relative to the standard SN divertor due to this increased asymmetry are not large.

Equilibria with $I_{p}$ lower than the design value of 11.8 MA are presented in Table 5.15. This sequence keeps $q_{\text {axis }}$ fixed at 0.8 . The first three entries have $\beta_{\text {pol }}$ fixed at 0.385 , while the final entry, shown in Fig. 5.11, has $\beta_{\text {pol }}=3.0$, corresponding to the Troyon limit for this plasma current. Shape control is imposed by constraining $R_{x}=2.107 \mathrm{~m}$, $Z_{x}=1.845 \mathrm{~m}$, since control of the $95 \%$ shape allows a wide variation in $\mathrm{X}$-point position at low current. For fixed $B_{t}=9 \mathrm{~T}, \ell_{i}$ and $q_{95}$ increase with lower $I_{p}$. The current in PF1 is fixed in each equilibrium solution and varied linearly with $\mathrm{I}_{p}$.

\section{V.B.7. Summary of Equilibrium Capabilities}

This section has presented calculations showing what coil currents are needed to satisfy the performance requirements in the GRD dealing with producing plasma equilibria. The reference operating modes are the $\mathrm{DN}$ divertor, the $\mathrm{SN}$ divertor, and the inner wall limiter. The coil currents required to produce these, assuming realistic plasma conditions, are given in Tables 5.4, 5.5, 5.6, 5.13, and 5.14. Examining these currents shows the distributions to be well behaved: They do not involve large oscillating current patterns. In addition, the magnitudes and necessary time rates of changes of the currents that are called for have been shown to be within the engineering capability of the device.

A requirement of the GRD is that BPX be designed to accommodate flexibility in the magnetic configuration and operating mode. This is addressed in Tables 5.4, 5.9, 5.13, 5.14, and 5.15, which together show that a wide variety of different plasma equilibria are available with the same coil set. These include equilibria needed for high elongation operation with the X-point on the divertor surface, and equilibria for single-null operation with an extended neck plasma. Also included in this demonstration are equilibria needed for discharges involving dynamic switching between SN top and bottom, DN, and limiter, or involving multiple divertor sweeps. In addition, it has been demonstrated that a wide range of plasma pressures and a variety of current profile shapes can be handled in this device, including those well outside the expected range. While the engineering analysis of the full range of flexibility has not been completed, it is clear that the BPX PF system as described here is capable of considerable flexibility from an equilibrium standpoint.

\section{V.C. PLASMA STARTUP}

BPX presents unique problems for the design of the plasma startup phase, particularly in producing the magnetic field conditions necessary to achieve breakdown and to ensure the stability of the developing current channel. Due to the large thickness of the vacuum vessel, eddy currents cause significant perturbations of the fields from the PF windings. It will also be necessary to initiate the 

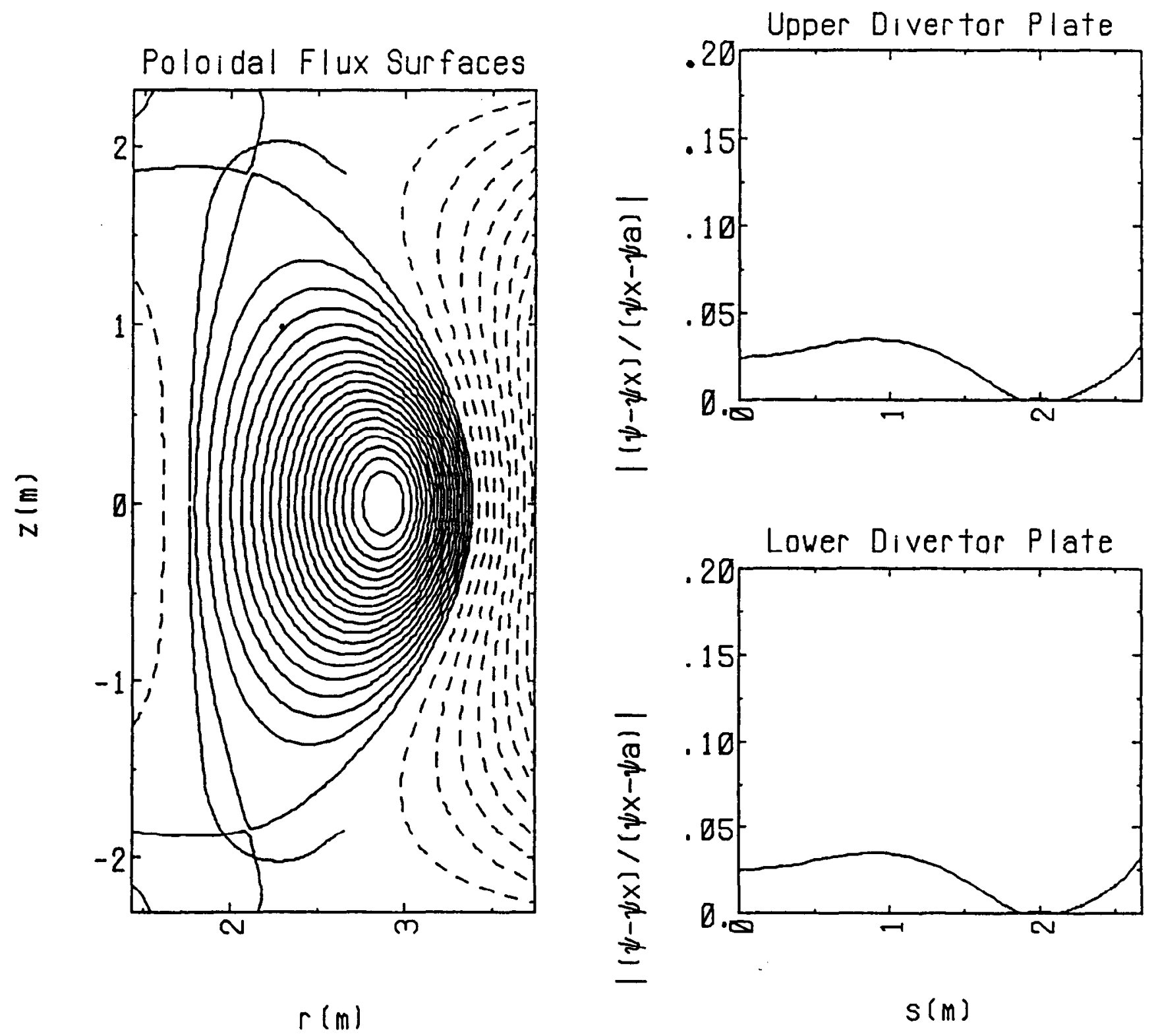

Fig. 5.11. Poloidal flux surfaces for $\beta_{p}=3.0$ equilibrium of Table 5.15. 
Jardin et al. MHD EQUILIBRIUM AND STABILITY

Table 5.13. Reverse-sweep 500-MW DN is Compared with the Standard 500-MW DN Divertor Sweep

\begin{tabular}{|c|c|c|c|c|}
\hline Case & Std. BOFT & R-S BOFT & Std. EOB & R-S EOB \\
\hline \multicolumn{5}{|l|}{ Plasma } \\
\hline$\kappa_{95}$ & 2.100 & 2.000 & 2.000 & 2.000 \\
\hline$\delta_{95}$ & 0.250 & 0.450 & 0.350 & 0.250 \\
\hline$R_{x}(\mathrm{~m})$ & 2.268 & 1.985 & 2.083 & 2.244 \\
\hline$Z_{x}(\mathrm{~m})$ & 1.891 & 1.784 & 1.866 & 1.859 \\
\hline$\Delta R_{e}(\mathrm{~m})$ & -0.007 & -0.007 & -0.003 & 0.000 \\
\hline \multicolumn{5}{|l|}{ Strike-Points (m) } \\
\hline$R_{\text {isp }}$ & 2.105 & 1.927 & 1.997 & 2.067 \\
\hline$Z_{\text {isp }}$ & 1.984 & 1.801 & 1.904 & 1.962 \\
\hline$R_{\text {osp }}$ & 2.346 & 2.035 & 2.152 & 2.346 \\
\hline$Z_{o s p}$ & 2.018 & 1.939 & 2.004 & 2.019 \\
\hline$d x_{i s p}(\mathrm{~m})$ & 0.188 & 0.060 & 0.094 & 0.239 \\
\hline$d x_{\text {osp }}(\mathrm{m})$ & 0.149 & 0.163 & 0.154 & 0.190 \\
\hline$\beta_{\text {pol }}$ & 0.210 & 0.210 & 0.385 & 0.385 \\
\hline$\ell_{i} / 2$ & 0.350 & 0.350 & 0.432 & $0, .432$ \\
\hline$q_{95}$ & 3.244 & 3.344 & 3.448 & 3.199 \\
\hline \multicolumn{5}{|l|}{ Coil Currents (MA) } \\
\hline PF1 & 10.582 & 13.526 & 15.975 & 14.260 \\
\hline PF2 & 5.722 & 0.191 & -0.104 & 3.546 \\
\hline PF3 & -4.220 & -9.135 & -1.678 & -0.894 \\
\hline PF4 & -6.684 & -5.365 & -3.978 & -4.876 \\
\hline PF5 & 1.835 & 3.884 & 0.343 & -0.105 \\
\hline PF6 & 0.978 & 1.466 & 1.566 & 1.256 \\
\hline PF7 & 3.904 & 2.757 & 4.497 & 4.840 \\
\hline \multicolumn{5}{|l|}{ Flux, Stored Energy } \\
\hline Flux (V-s) & 33.000 & 33.000 & 39.200 & 39.200 \\
\hline$W_{P F}(\mathrm{GJ})$ & 1.194 & 1.301 & 1.308 & 1.371 \\
\hline
\end{tabular}


Table 5.14. Reference SN, Diverted Equilibria at BOFT and EOB Compared to Those of a Long Divertor Mode (LDM) in which the X-points are Displaced by $d Z_{x}=-0.1 \mathrm{~m}$ from the Reference Case

\begin{tabular}{|l|r|r|r|r|}
\hline Case & Ref. BOFT & LDM BOFT & Ref. EOB & LDM EOB \\
\hline Plasma & & & & \\
$Z_{\text {axis }}(\mathrm{m})$ & -0.002 & -0.095 & -0.012 & -0.098 \\
$Z_{x, u}(\mathrm{~m})$ & 1.891 & 1.791 & 1.866 & 1.766 \\
$Z_{x, l}(\mathrm{~m})$ & -1.900 & -2.000 & -1.972 & -2.072 \\
$\Delta R_{e, u}(\mathrm{~m})$ & 0.004 & -0.016 & -0.009 & -0.026 \\
$\Delta R_{e, l}(\mathrm{~m})$ & 0.008 & 0.023 & 0.007 & 0.020 \\
$d x_{\text {isp }}(\mathrm{m})^{*}$ & 0.183 & 0.281 & 0.094 & 0.157 \\
$d x_{\text {osp }}(\mathrm{m})^{*}$ & 0.151 & 0.252 & 0.155 & 0.274 \\
Coil Currents (MA) & & & & \\
PF1 & 11.424 & 11.504 & 15.785 & 16.052 \\
PF2 & 3.972 & 4.034 & -0.126 & -0.633 \\
PF3U & -2.022 & -4.108 & -1.877 & -3.547 \\
PF3L & -9.776 & -7.390 & -0.163 & 1.735 \\
PF4U & -7.216 & -7.525 & -3.720 & -3.288 \\
PF4L & -3.117 & -3.679 & -2.815 & -3.144 \\
PF5 & 1.913 & 2.118 & -0.085 & -0.015 \\
PF6U & 1.168 & 0.894 & 1.552 & 0.842 \\
PF6L & -0.062 & 0.368 & 0.637 & 1.279 \\
PF7 & 3.998 & 3.921 & 4.775 & 4.762 \\
\hline
\end{tabular}

${ }^{*} d x_{i s p}$ and $d x_{\text {osp }}$ are distances from X-point to inner and outer strike-points, respectively. 
Jardin et al. MHD EQUILIBRIUM AND STABILITY

Table 5.15. Sequence of Fixed X-Point Equilibria with $q_{\text {axis }}=0.8$ and Variation in the Plasms Current

\begin{tabular}{|l|r|r|r|r|}
\hline Plasma & -4.000 & -8.000 & -11.800 & -3.933 \\
$I_{p}$ (MA) & 8.799 & 4.680 & 3.372 & 9.596 \\
$q_{95}$ & 0.385 & 0.385 & 0.385 & 3.000 \\
$\beta_{p}$ & 0.235 & 0.951 & 2.060 & 1.852 \\
$\beta_{\%}$ & 0.643 & 0.490 & 0.408 & 0.598 \\
$\ell_{i} / 2$ & 1.886 & 1.942 & 2.000 & 1.824 \\
$\kappa_{95}$ & 0.192 & 0.312 & 0.350 & 0.245 \\
$\delta_{95}$ & -0.003 & -0.005 & -0.004 & -0.019 \\
$\Delta R_{e}$ (m) & -0.003 & -0.005 & -0.004 & -0.019 \\
Coil Currents (MA) & 5.424 & 10.848 & 16.000 & 3.268 \\
PF1 & 1.202 & -0.721 & 1.954 & 1.253 \\
PF2 & -3.675 & -1.816 & -0.904 & -2.378 \\
PF3 & 0.471 & -2.487 & -4.580 & 0.861 \\
PF4 & -0.668 & -0.105 & 0.852 & -1.279 \\
PF5 & -1.201 & 0.474 & 2.090 & -1.707 \\
PF6 & 2.837 & 3.536 & 3.941 & 4.303 \\
PF7 & & & & \\
Flux, Stored Energy & 13.385 & 26.035 & 40.925 & 15.776 \\
Flux (V-s) & 0.266 & 0.627 & 1.378 & 0.513 \\
WPF (GJ) & & &
\end{tabular}


plasma during the rampup of the toroidal field, which may generate additional stray fields and will affect the stability of the plasma column. Furthermore, the long penetration time for external fields through the vacuum vessel means that real-time feedback control of the plasma equilibrium will not be possible until the plasma current has risen to a significant level. Until the feedback control is established, the plasma equilibrium must be controlled by preprogrammed currents in the external windings. Thus, the stray magnetic fields must be accurately modeled both in space and time to enable the startup conditions to be designed. Conservative requirements for plasma startup established to overcome these design difficulties are maintained because, due to the low shot rate and limited fatigue life, the initiation of the discharge in BPX must be very reliable.

The specifications for the startup conditions have been reached by adapting experience in previous tokamaks to the unique constraints of the BPX device. In common with most present-day tokamaks, it is planned to use inductive startup of the discharge. Prior to breakdown, the ohmic heating transformer will be charged to provide the initial poloidal flux bias needed for the planned discharge, and an appropriate pattern of currents will be set up in the other PF windings. These currents will be rapidly changed by a combination of power supplies and high-power switches to induce a toroidal electric field and, simultaneously, a null in the poloidal magnetic field inside the vacuum vessel. Combined with a sufficient toroidal magnetic field to confine the electrons, these conditions will produce an avalanche breakdown of the pre-filling gas in the vessel. A current channel will then develop and be maintained in equilibrium if the subsequent time evolution for the loop voltage and the poloidal magnetic field is appropriate. At this stagc, it is not planned to use RF assistance during the breakdown phase. The requirements for the breakdown loop voltage pulse are based on experience in present tokamaks of similar size to BPX. An unusual feature of the requirements is a two-tiered specification for the magnitude of this pulse. This follows from the assumption that if the tokamak is not operating at its optimum level of plasma cleanliness, discharges at the maximum plasma current will not be run. Experience suggests that the loop voltage required to achieve reliable initiation is lower when the level of impurities in the plasma is lower. Thus, the requirement to be able to produce an inductive loop voltage of $20 \mathrm{~V} /$ turn for discharges with a maximum plasma current of $5 \mathrm{MA}$, can be relaxed to $12 \mathrm{~V} / \mathrm{turn}$ when the ohmic heating transformer solenoid is fully precharged in preparation for a discharge at the maximum plasma current. This reduces the requirements for the power supplies and switches. For full-current discharges, the toroidal magnetic field is specified to be at least $5 \mathrm{~T}$ at plasma initiation. This requirement is conservatively set to ensure the reliability of breakdown and also an adequate margin of stability for the evolving plasma equilibrium.

During the initiation pulse of the toroidal electric field, it is also necessary to produce a suitable null in the poloidal magnetic field. In BPX, this null will be produced dynamically by balancing the $\mathrm{PF}$ components from several PF windings and the large eddy currents induced in the structure surrounding the plasma by the changing $\mathrm{PF}$ and $\mathrm{TF}$ currents. To allow the breakdown avalanche to develop and form a current channel, it is specified that the null point should remain within a circular region of radius $0.1 \mathrm{~m}$ in the poloidal cross section for a period of at least $5 \mathrm{~ms}$ during the rise of the loop voltage pulse. The region within which the null develops must be able to be positioned on the midplane at any radius between the inner and outer limiter surfaces to provide flexibility for controlling the subsequent evolution of the discharge. For example, it may be desired to "grow" the plasma from either limiter surface to produce optimal current penetration and to control impurities. The breakdown conditions should persist for deviations of the currents in the PF windings from their nominal values by up to $0.5 \%$, the estimated reproducibility of the power supply currents. It must also be possible to compensate for (uniform) stray magnetic fields in the vacuum vessel of up to $5 \mathrm{mT}$ magnitude arising from deviations of the TF and PF coils from their nominal positions or from other sources of stray fields.

After the avalanche breakdown phase, the poloidal field must evolve to provide MHD equilibrium as the plasma current increases. In BPX, the equilibrium field will be controlled by preprogrammed currents in the PF windings until the plasma current has risen to $\sim 0.1 \mathrm{MA}$ and active feedback of the position can be started. Immediately following the breakdown, the plasma will be potentially unstable to either radial or axial displacements depending on the sign of the poloidal field gradient in the vicinity of the breakdown null. However, experience on existing tokamaks has shown that this instability is not a problem provided that the field gradient is not too large and that the field evolves to provide ideal stability in both radial and axial directions by the time that the plasma current has reached a level of 20 to $50 \mathrm{kA}$, which will occur within about $10 \mathrm{~ms}$ of breakdown. The field gradient in BPX at the time of breakdown is specified to be adjustable within 
the range $\pm 2 \mathrm{mT} / \mathrm{m}$ by varying the $\mathrm{PF}$ coil currents. This will permit the selection of the most appropriate gradient to provide reliable startup for the particular operational conditions. To calculate the preprogrammed currents in the PF windings following breakdown, it will be assumed, again on the basis of experience with present tokamaks of size similar to BPX, that the plasma extends to the limiter, has zero pressure and that the internal inductance $\ell_{i}$ rises from 0.5 at breakdown to 1.0 after $0.2 \mathrm{~s}$. The resistive component of the loop voltage will be taken to be the applied voltage for $20 \mathrm{~ms}$ after breakdown and then to fall exponentially to $1 \mathrm{~V} /$ turn with a time constant of $0.1 \mathrm{~s}$. Since the actual breakdown may evolve differently due to the unique first-wall conditions in BPX, the robustness of the initiation phase must be tested by modeling the discharge evolution for variations of plasma parameters, resulting from changes in transport and radiation, over likely ranges.

\section{V.D. SHAPE CONTROL}

The proposed shape control system on BPX uses the seven independent external coils, PF1 to PF7, to control the position of a small number $(\leq 5$, for a symmetric, diverted plasma) of critical points, and possibly flux parameters, in the magnetic field geometry. Shape control is accomplished on the slow time scale ( $\geq 100 \mathrm{~ms}$ ) and consists of two subproblems:

1. detection of the plasma boundary from flux loop measurements

2. determination of the correction currents using shape control matrices.

In this section, we consider computational methods for solving the shape control problem and apply them to an earlier BPX design $\left(R_{o}=2.1 \mathrm{~m}\right.$, $I_{p}=11.0 \mathrm{MA}, B_{t}=10 \mathrm{~T}$, GEM-29 PF set), using flux measurements from a $\kappa=2.1$, double-null, diverted MHD equilibrium at BOFT.

\section{V.D.1. Detection of the Plasma Boundary}

The boundary of a double-null, diverted plasma is characterized by four shape parameters. Two of these are radii of the inner and outer edge, $R_{1}$ and $R_{2}$, on the plasma midplane. Options for the two remaining shape control parameters include (a) coordinates of the X-point $\left(R_{x}, Z_{x}\right)$, (b) distance along the divertor plate to the inner and outer strike-points $\left(d x_{i s p}, d x_{\text {osp }}\right)$, or (c) $95 \%$ flux surface shape parameters $\left(\kappa_{95}, \delta_{95}\right)$. Since computing all of these parameters depends on a knowledge of the separatrix flux surface, the first step in determining the plasma boundary is to approximately determine the value of the poloidal flux at the $\mathrm{X}$ point.

We approximate the poloidal flux function in the $\mathrm{X}$-point region by

$$
\Psi=\Psi^{0}+c_{1} \phi_{1}+\cdots+c_{7} \phi_{7}
$$

where $\Psi^{0}$ is an approximation to $\Psi$ based on a flament representation of the plasma and coil currents,

$$
\Psi^{0}=\Psi^{\text {plasma }}+\Psi^{\text {coils }},
$$

and the $\phi_{j}$ are basis functions used to expand $\Psi$ in a Taylor series about some guess for the $\mathrm{X}$-point $\left(R_{x}, Z_{x}\right)$, while requiring that they satisfy the homogeneous equilibrium equation $\Delta^{*} \phi_{j}=0$ to leading order in an aspect ratio expansion. These functions are listed in Table 5.16. The flux and field at the loops are related to the plasma and coil currents by the Green's function matrices $A^{L P}$ and $A^{L C}$, respectively. The flux loop equations for $J$ are

$$
A^{L P} J=b^{L}-A^{L C} I
$$

where the vector $b^{L}$ consists of the flux and field measurements, $b^{L}=\left[\Psi^{L}, B_{r}^{L}, B_{z}^{L}\right]$. The plasma current approximation $J$ is used in Eq. (5.6) and is based on a filament model, and the coil current vector $I$ is assumed to be known. Equation (5.7) is first solved for $J$ using all of the flux loop data, and Eq. (5.5) is then solved for the $c_{j}$ using only the measurements from flux loops nearest the X-point.

Figure 5.12 illustrates the approximation of the poloidal flux function in the X-point region that is computed using only the linear combination of basis functions [i.e., Eq. (5.5) with $\Psi^{0}=0$ ]. The resulting $X$-point position, obtained by solving the equations $\partial \Psi / \partial R=0$ and $\partial \Psi / \partial Z=0$, is $2.4 \mathrm{~cm}$ from the actual equilibrium X-point when only the seven loop locations nearest the X-point are used. If the flux due to the coil currents and plasma filament model $\Psi^{0}$ is included in Eq. (5.5), this error in estimating the equilibrium value of the X-point position is reduced to $0.3 \mathrm{~cm}$ (Fig. 5.13).

Newton's method is used to solve $\partial \Psi / \partial R=0$ and $\partial \Psi / \partial Z=0$ for the coordinates of the $\mathrm{X}$ point. The representation Eq. (5.5) is evaluated at $\left(R_{x}, Z_{x}\right)$ to find the separatrix flux value $\Psi_{x}$ and used to locate divertor strike-points by solving $\Psi(s)-\Psi_{x}=0$ for points $s$ on the known divertor plate surface. Finally, flux loop measurements near the midplane are used to approximate radial coordinates of the plasma edge on the midplane for which $\Psi\left(R_{1,2}\right)-\Psi_{x}=0$. Computing divertor strike-points is more accurate than computing the $\mathrm{X}$-point position, where the poloidal flux function is relatively flat. Computing the midplane edge coordinates accurately depends on whether nearby flux measurements are available. 
Table 5.16. Basis Functions Used to Represent Poloidal Flux in Neighborhood of $\left(R_{x}, Z_{x}\right)$

\begin{tabular}{|c|l|}
\hline No. & \multicolumn{1}{|c|}{ Function } \\
\hline 1 & 1 \\
2 & $\left(R-R_{x}\right)+\left(Z-Z_{x}\right)^{2}\left[1-\left(R-R_{x}\right) / R_{x}\right] / 2 R_{x}$ \\
3 & $Z-Z_{x}$ \\
4 & $\left(R-R_{x}\right)^{2}-\left(Z-Z_{x}\right)^{2}+\left(R-R_{x}\right)\left(Z-Z_{x}\right)^{2} / R_{x}$ \\
5 & $\left(R-R_{x}\right)\left(Z-Z_{x}\right)\left[1+\left(R-R_{x}\right) / 2 R_{x}\right]$ \\
6 & $\left(Z-Z_{x}\right)\left[\left(Z-Z_{x}\right)^{2}-3\left(R-R_{x}\right)^{2}\right]$ \\
7 & $\left(R-R_{x}\right)\left[\left(R-R_{x}\right)^{2}-3\left(Z-Z_{x}\right)^{2}\right]$ \\
\hline
\end{tabular}

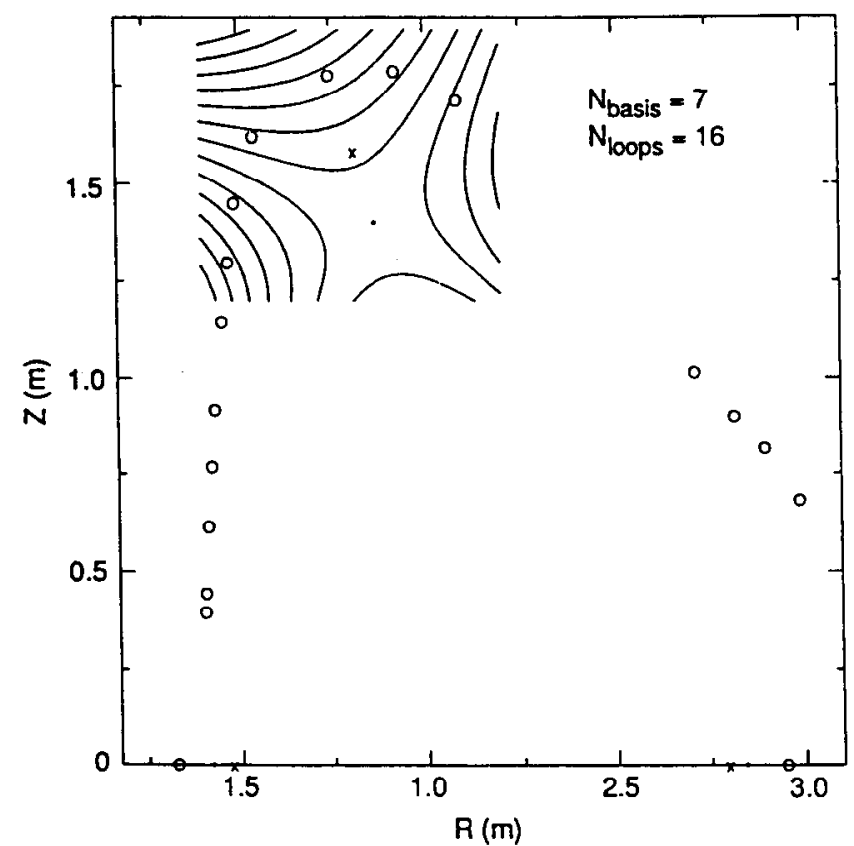

Fig. 5.12. Poloidal flux approximation in the Xpoint region using Eq. (5.5) with $\Psi^{0}=0$ and only the seven flux loop measurements nearest the $\mathrm{X}$ point.

\section{V.D.2. Plasma Shape Control Matrices}

If, for example, we wish to control the plasma position and X-point, the methods of the previous section are used to compute an error vector of the form

$$
\epsilon=\left[\Delta R_{1}, \Delta R_{2}, \Delta R_{x}, \Delta Z_{x}\right]
$$

where $\Delta R_{1}$ and $\Delta R_{2}$ are the differences between the measured and reference radial coordinates of the midplane inner and outer separatrix surface and the reference values, respectively. The plasma shape control matrix $A$ provides a linear relationship between this crror vector and the vector of

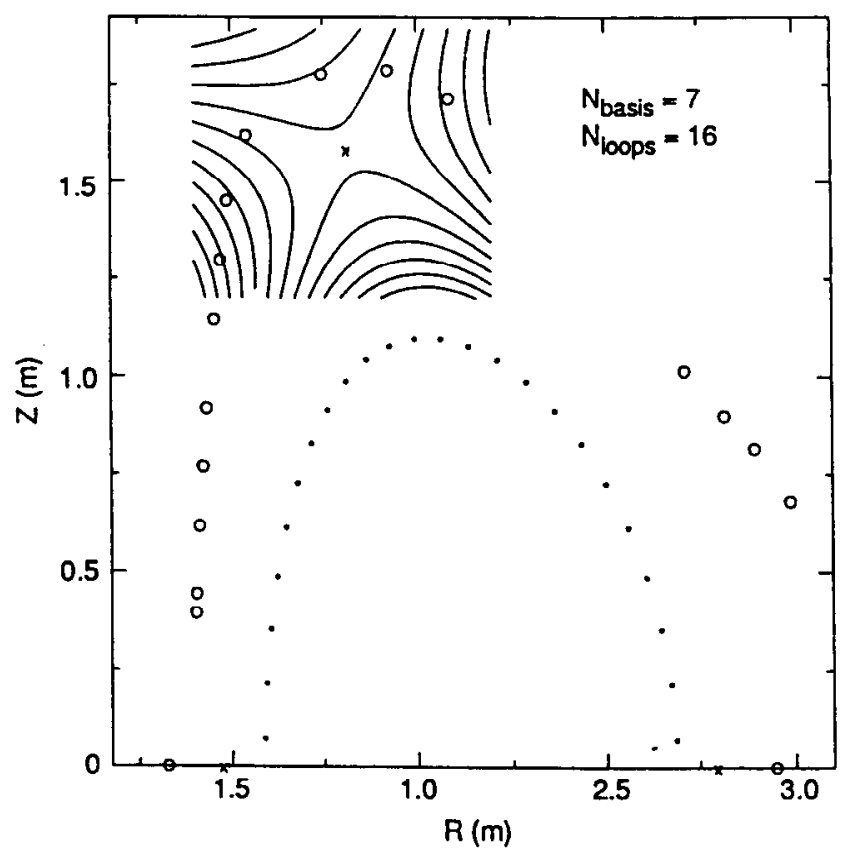

Fig. 5.13. Poloidal flux approximation in the Xpoint region using Eq. (5.5) with the plasma filament model for $\Psi^{0}$ based on all 16 flux loop measurements and the linear combination of basis functions based on the seven flux loops nearest the Xpoint.

correction currents, i.e.,

$$
\delta I=A \epsilon,
$$

where

$$
\delta I=\left[\Delta I_{1}, \cdots, \Delta I_{7}\right] .
$$

If all seven independent PF currents are used for shape control, the control matrix is computed in the following way:

1. Compute a reference MHD equilibrium solution with prescribed plasma profiles. 
2. Vary each PF current individually about the reference equilibrium values and determine the change in each shape parameter in a set of $M$ additional equilibria. For example, each of the seven PF currents may be varied by $\pm d I$, a given fraction of the total plasma current.

3 . Record the error vectors $\epsilon_{j}, j=1 \cdots M$, associated with these equilibrium solutions, i.e., the difference between the calculated control parameters and those for the reference equilibrium.

4. Set $a_{i} \cdot \epsilon_{j}=d I_{i j}, i=1 \cdots 7 ; j=1 \cdots M$, where $d I_{i j}$ is the change in coil current $i$ for equilibrium $j$. Solve a $7 M \times 28$ system of linear equations for the elements $a_{i, k}$ of the row vectors $a_{i}$ of the $7 \times 4$ control matrix $A$. Typically, $M>4$, and a least-squares solution is obtained.

A typical control matrix calculated in this fashion for a baseline equilibrium at BOFT is given in Table 5.17. The shape control matrix described

Table 5.17. The Plasma Shape Control Matrix $A$ (MA/m)

\begin{tabular}{|c|r|r|r|r|}
\hline PF & $R_{1}$ & $R_{2}$ & $R_{x}$ & $Z_{x}$ \\
\hline 1 & -69.04 & -1.47 & 25.84 & -6.27 \\
2 & 39.04 & -8.88 & -18.20 & 15.14 \\
4 & 23.34 & 5.13 & -7.91 & -2.73 \\
4 & -19.56 & 16.40 & 19.35 & -26.89 \\
5 & 9.05 & -24.98 & -0.64 & 22.49 \\
6 & 10.89 & -11.15 & -4.17 & 14.62 \\
7 & -6.80 & 24.07 & 2.25 & -15.79 \\
\hline
\end{tabular}

here is not unique since seven currents are used to correct four errors. Further considerations could be used in determining $A$, including the minimization of stored energy. Flux linkage may be used as an additional control parameter. Also, note that control matrices vary with the plasma profiles prescribed in the equilibrium calculations. In a time-dependent simulation, such as provided by the TSC code, sequences of control matrices will be referenced to include the dependence on profile parameters, e.g., poloidal beta and internal inductance.

\section{V.D.3. An Alternative Method}

An alternative control method has been independently developed and demonstrated to work in a
TSC simulation of a BPX discharge. ${ }^{8}$ The algorithm consists of four basic elements: ${ }^{9}$ The first is a matrix $A$, which converts the vector of measurements (coil currents, flux loops, $B$ probes, etc.) into a vector of error signals (flux errors, magnetic ficld crrors, positional error, plasma current error, etc.). This matrix depends on a number of preprogrammed functions (coordinates of boundary points, desired plasma current, etc.). It involves the reconstruction of the plasma current in the form of a set of finite elements, and it computes flux and magnetic field errors at the preprogrammed boundary points. The second element consists of a number of proportional-integral-derivative controllers that act on the error signals and whose gain coefficients are adjusted for optimum closedloop stability. The third element is again a matrix, $M^{-1}$, which calculates the rate of change of the PF coil currents, such as to obtain the maximum possible error reduction. This is achieved by minimizing a cost functional of the form

$$
C=\Sigma_{i} \alpha_{i} \epsilon_{i}^{2}+d \Sigma_{j} \beta_{j} I_{j}^{2}
$$

where $\epsilon_{i}$ are flux errors or magnetic field errors, $I_{j}$ are coil currents, and $\alpha_{i}, \beta_{j}$ are weighting coefficients. The $\alpha_{i}$ 's are usually assumed to be unity, whereas the $\beta_{j}$ 's are chosen such that the second term on the right side of Eq. (5.9) becomes proportional to the total power dissipation in the PF coils. The parameter $d$ controls the trade-off between shape accuracy and power dissipation. The fourth element (matrix $L$ ) finally computes the coil voltages that are necessary to produce the desired rate of change of the coil currents.

This method differs from that described in Secs. V.D.1 and V.D. 2 in several respects. The most notable is that it does not require any preprogrammed coil currents, but it directly computes the voltages to be applied to individual coils that will minimize the cost function [Eq. (5.9)]. It utilizes three matrices, $A, M^{-1}$, and $L$, which, in general, will vary with time since they depend on the preprogrammed shape functions. However, they do not depend on the plasma parameters $\beta_{p}, \ell_{i}$, $q_{\text {axis }}$, etc., and do not require MHD equilibrium calculations for their generation.

An illustration of two time points in a BPX discharge sequence calculated using this method is given in Fig. 5.14. The open circles are the preprogrammed boundary points that are used to specify the desired plasma boundary. Preliminary conclusions are that this method appears quite promising, and we are in the process of comparing its relative merits with those of the method discussed at the beginning of the section. 


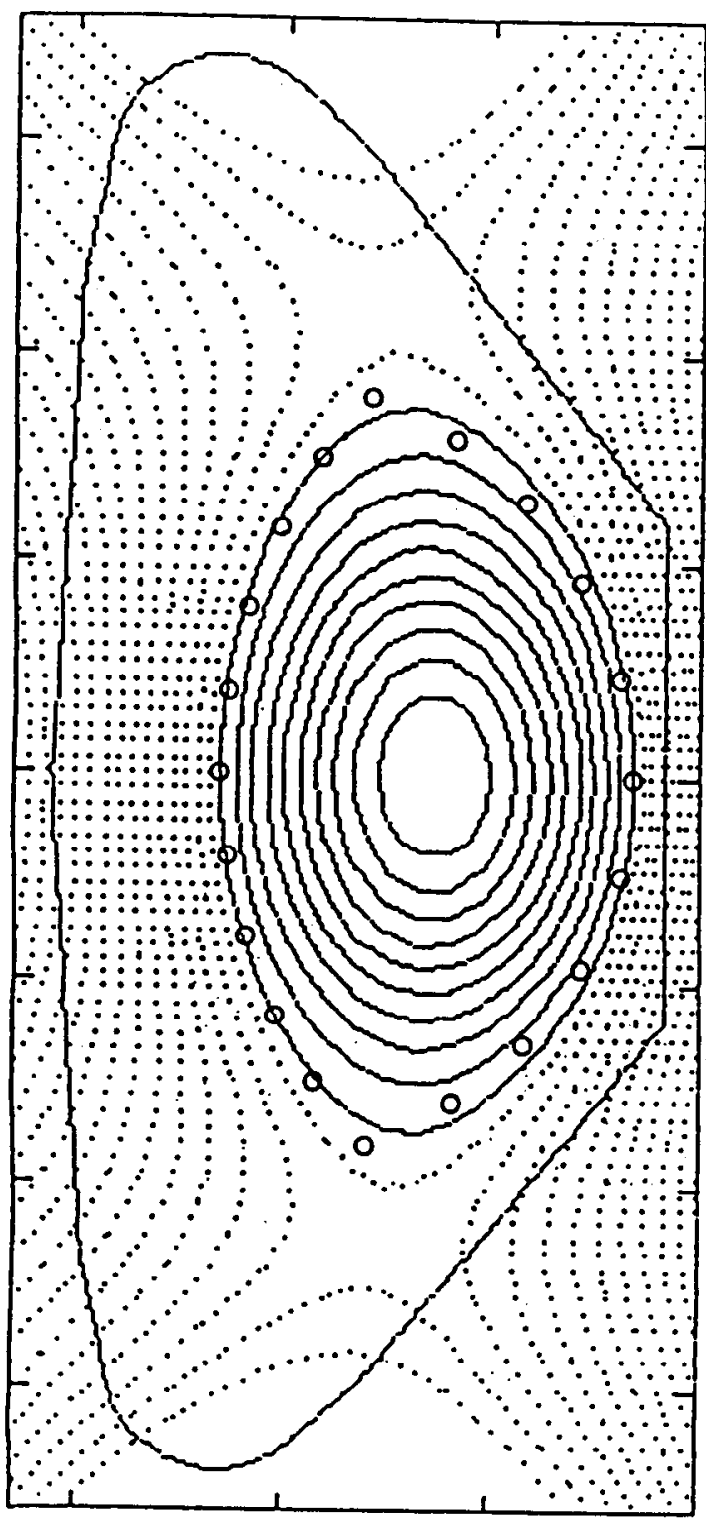

$1.87 \mathrm{sec}$

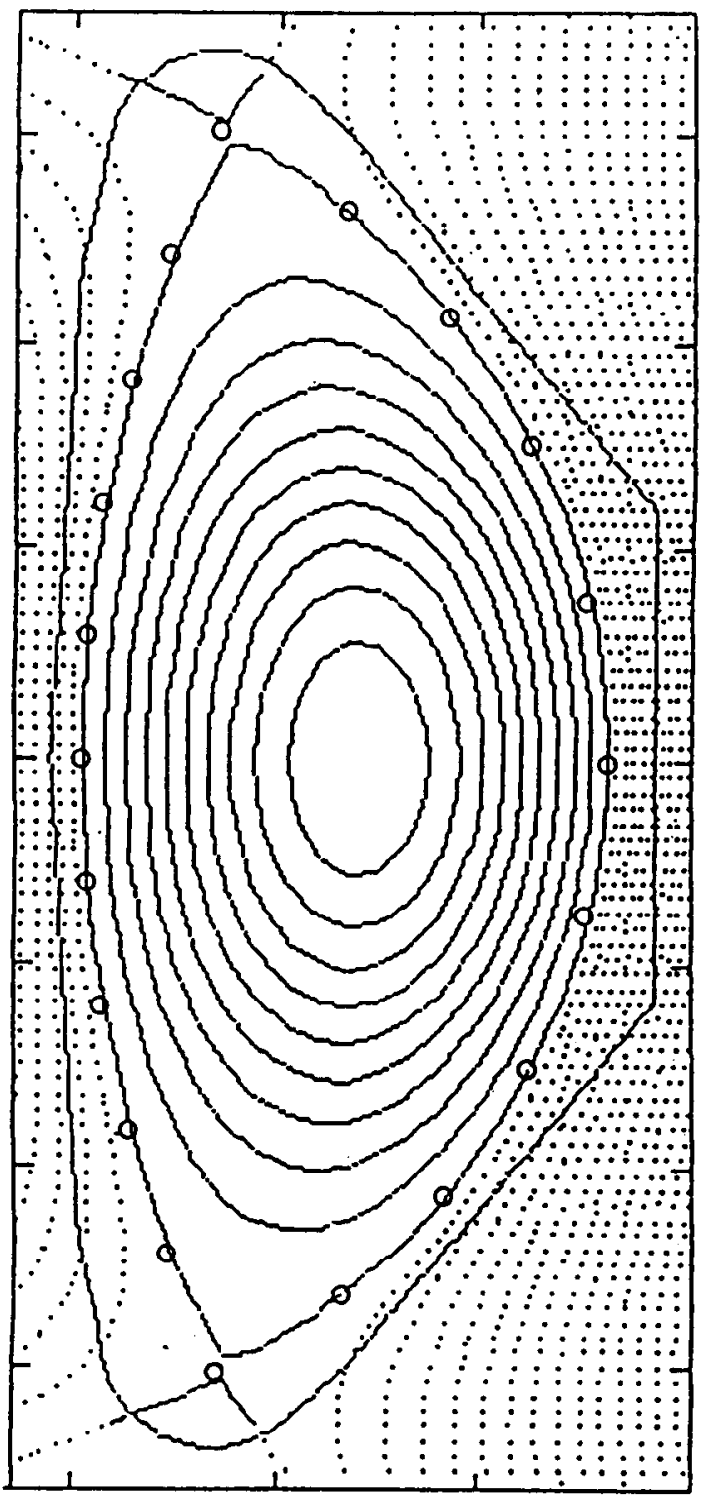

$6.42 \mathrm{sec}$

Fig. 5.14. Flux plots at $t=1.87$ and $t=6.42 \mathrm{~s}$ of a discharge sequence calculated using the method of Ref. 9. Positions of the preprogrammed boundary points are indicated by open circles. 


\section{V.E. VERTICAL STABILITY AND CONTROL}

\section{V.E.1. Vertical Stability}

Vertical stability of BPX plasmas is assessed by determining the growth time (reciprocal of the growth rate) for plasma configurations inside the toroidally continuous vacuum vessel structure. The effectiveness of the vessel for slowing down the plasma vertical motion depends on the plasma current distribution, the proximity of the plasma to conductors, the electrical resistance of the conductors, and the plasma elongation. The total plasma current does not influence the stability; however, it is very important when determining feedback control system requirements for coil currents and voltages. The vacuum vessel is the most critical structure since it lies the closest to the plasma and is toroidally continuous. The primary tool used in analyzing the vertical stability and control of BPX plasmas is the Tokamak Simulation Code ${ }^{2}$ (TSC), a free-boundary axisymmetric simulation code that models the resistive time scale evolution of plasmas, including the interaction with timevarying poloidal and toroidal magnetic fields, and with passive and active axisymmetric circuit elements. In addition, selected calculations have been performed independently using both the NOVAW (Ref. 11) and the LLNL code, ${ }^{12}$ confirming the TSC results.

Shown in Fig. 5.15 are the plasma flux surface contours inside the TSC representation of the vacuum vessel, for beginning of flattop (BOFT) and end of burn (EOB). Also given are the poloidal flux and current density across the midplane. It is clear that the current density is broader at BOFT than $\mathrm{EOB}$, with $\ell_{i} / 2=0.34$ and 0.40 , respectively. The elongations of the two plasmas are both $\kappa=$ 2.0 at the $95 \%$ flux surface. In the absence of an active feedback system, the vertical growth times are $21.0 \mathrm{~ms}$ (BOFT) and $16.5 \mathrm{~ms}$ (EOB). This illustrates the effect of the current profile; broader profiles are generally more stable to axisymmetric displacements than are peaked profiles. Part of this effect is due to the fact that the broader profile places more plasma current closer to the conductor, increasing its stabilizing effect. The remaining factor is that broader profiles require less shaping field strength to produce a given boundary shape, and thus reduce the drive term for the vertical instability.

To further quantify this effect, the vertical growth times were determined for several different values of $\ell_{i} / 2$. The results are plotted in Fig. 5.16 for plasmas with $\kappa_{95}=2.0$. The variation of growth time with $\ell_{i} / 2$ is considerable. For peaked current profiles, the plasma could move too fast for the feedback control coils to provide a restoring radial field. If such profiles exist during the discharge the elongation must be reduced below $\kappa_{95}=2.0$.

It is of interest to determine the effect on vertical stability of BPX plasmas with elongations larger than the nominal flattop $\kappa_{95}=2.0$. A plasma with elongation $\kappa_{95}=2.2$ is shown in Fig. 5.17, at BOFT with $\ell_{i} / 2=0.34$. The growth time for this plasma is $19.0 \mathrm{~ms}$. It would be expected that a significant drop in the growth time would take place for increased elongation. However, in this case, there is a partially offsetting stabilizing effect in that the plasma boundary has moved closer to the vessel, and since the current profile is broad, the plasma has a relatively long growth time. As the current density is made more peaked, the growth time would be expected to drop more rapidly than that shown in Fig. 5.16 for a $\kappa_{95}=2.0$ plasma. Although these calculations have not been made, previous work would indicate that for $\kappa_{95}=2.2$, the growth time at EOB, with $\ell_{i} / 2=0.4$, would be around $10.0 \mathrm{~ms}$, compared to $16.5 \mathrm{~ms}$ for the $\kappa_{95}=2.0$ plasma.

To examine the effectiveness of the vacuum vessel proximity to the plasma boundary, the vertical stability of full-current BPX plasmas was examined in a fictitious vacuum vessel. The fictitious vessel is identical to the true BPX vacuum vessel, except that the separation distance between the top/bottom sections of the vessel and the top/bottom of the plasma was increased by approximately $7.0 \mathrm{~cm}$. The growth times for plasma elongation $\kappa_{95}=2.0$ at BOFT, $\kappa_{95}=2.0$ at $\mathrm{EOB}$, and $\kappa_{95}=2.2$ at BOFT, in the presence of this modified vessel are $16.0,13.5$, and $15.5 \mathrm{~ms}$, respectively. Vertical stability was also examined for plasma configurations during the rampup and rampdown phases. The flux surface contours for these configurations are shown in Fig. 5.18, for times during the discharge of $1.0,2.0$, and $3.5 \mathrm{sec}-$ onds into the rampup, and 1.0 second into the rampdown. The plasma parameters are given in the figure. The plasmas have elongations of $\kappa_{95}=$ $1.3, \kappa_{95}=1.5, \kappa_{95}=1.8$, and $\kappa_{95}=1.95$, and the corresponding growth times are 25, 22, 19, and 15 $\mathrm{ms}$. The plasmas during rampup have long enough growth times to be controllable as there is a competition between elongation and distance between the plasma boundary and vacuum vessel.

It is also of interest to examine larger aspect ratio plasmas in the BPX device, obtained by reducing the minor radius of the plasma. One such plasma, with $R / a=4.0$, is shown in Fig. 5.17. The plasma is seen to be farther from the vessel and is found to be ideally unstable, with a growth time less than $1.0 \mathrm{~ms}$, much too fast to be controlled. This study is relevant to plasma growth scenarios since the 

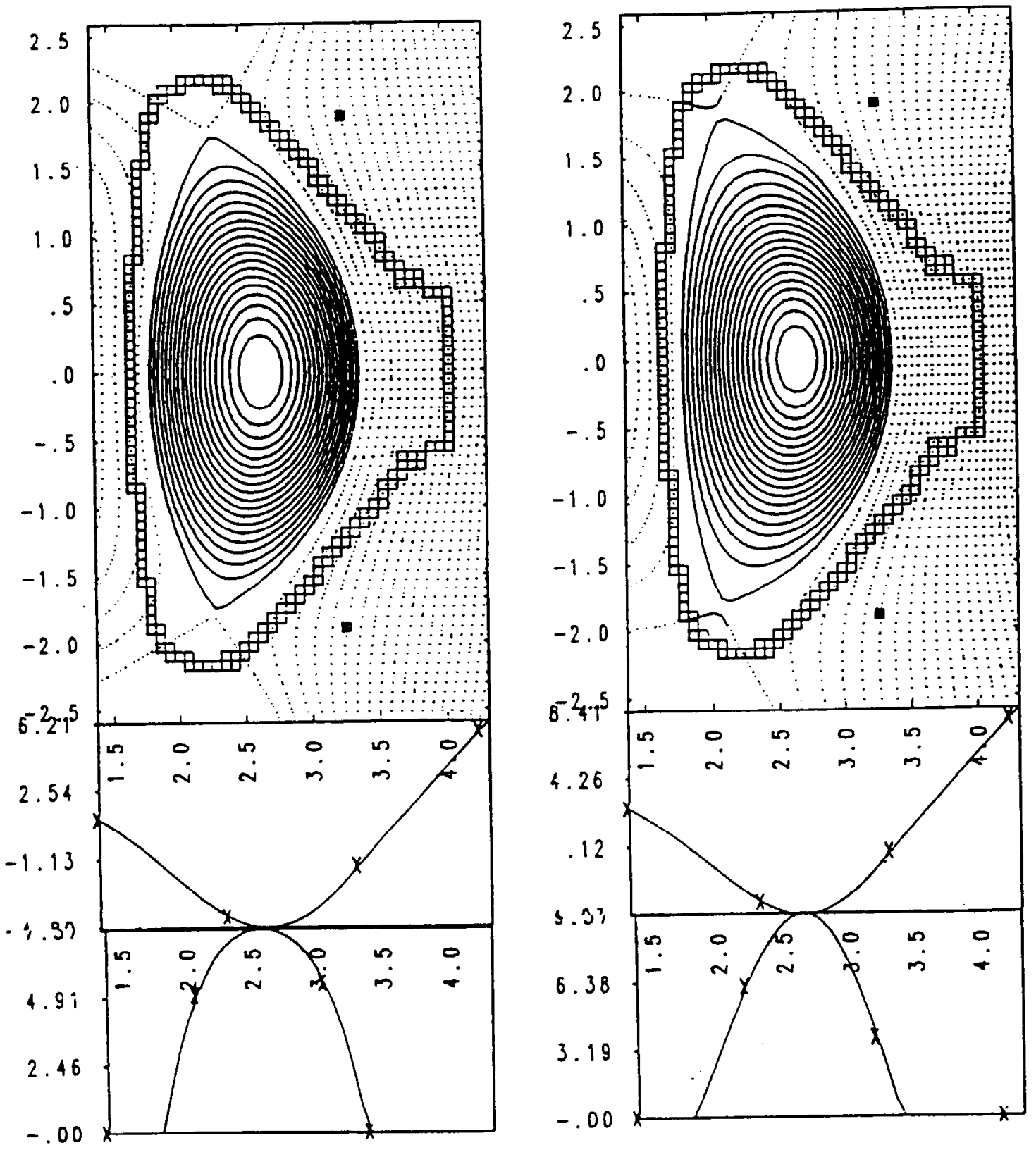

Fig. 5.15. Plasma flux surface contours inside the TSC representation of the vacuum vessel for (a) beginning of flattop and (b) end of burn. 
Jardin et al. MHD EQUILIBRIUM AND STABILITY

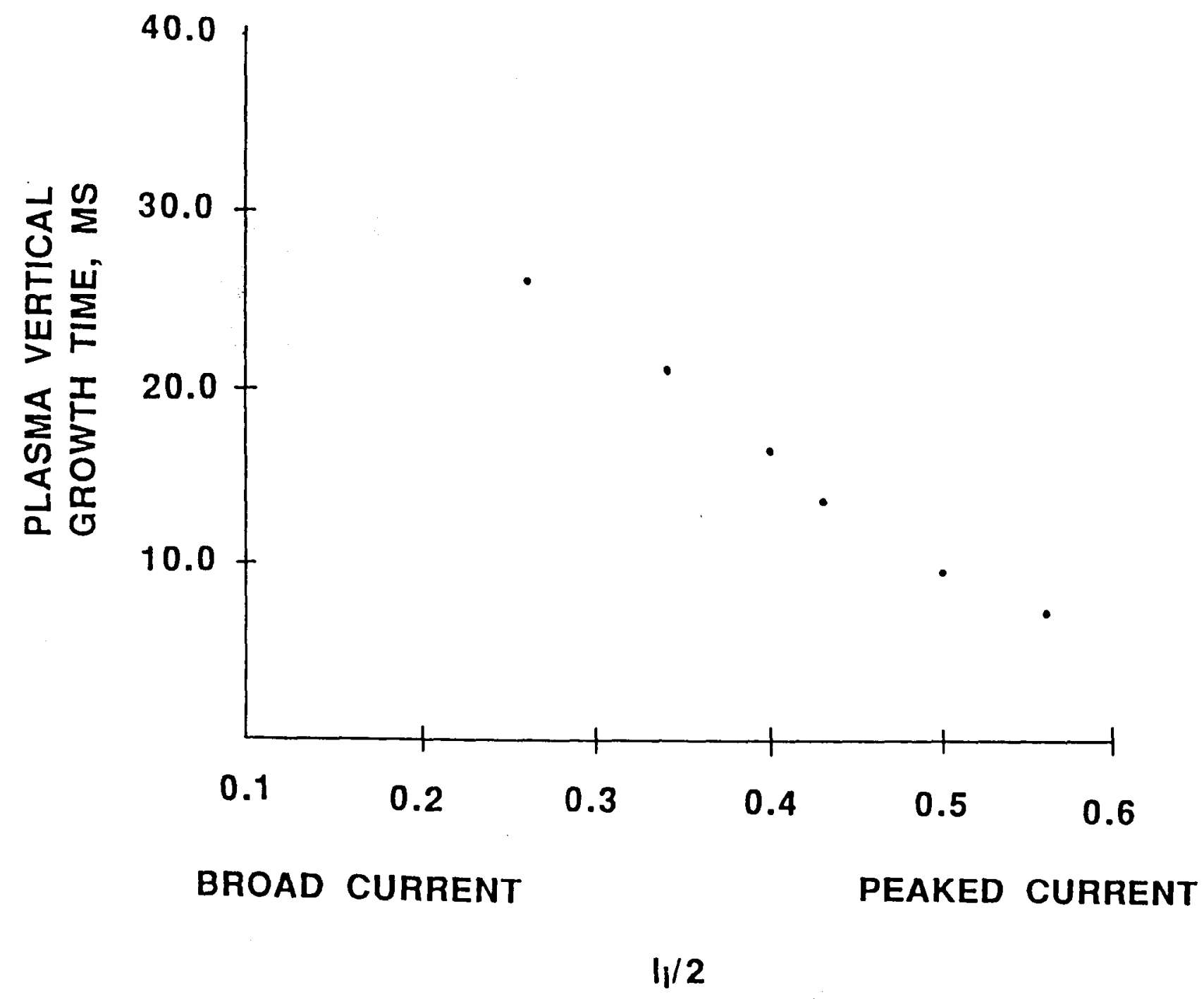

Fig. 5.16. Variation of the plasma vertical growth time with current profile (internal inductance per unit length). 

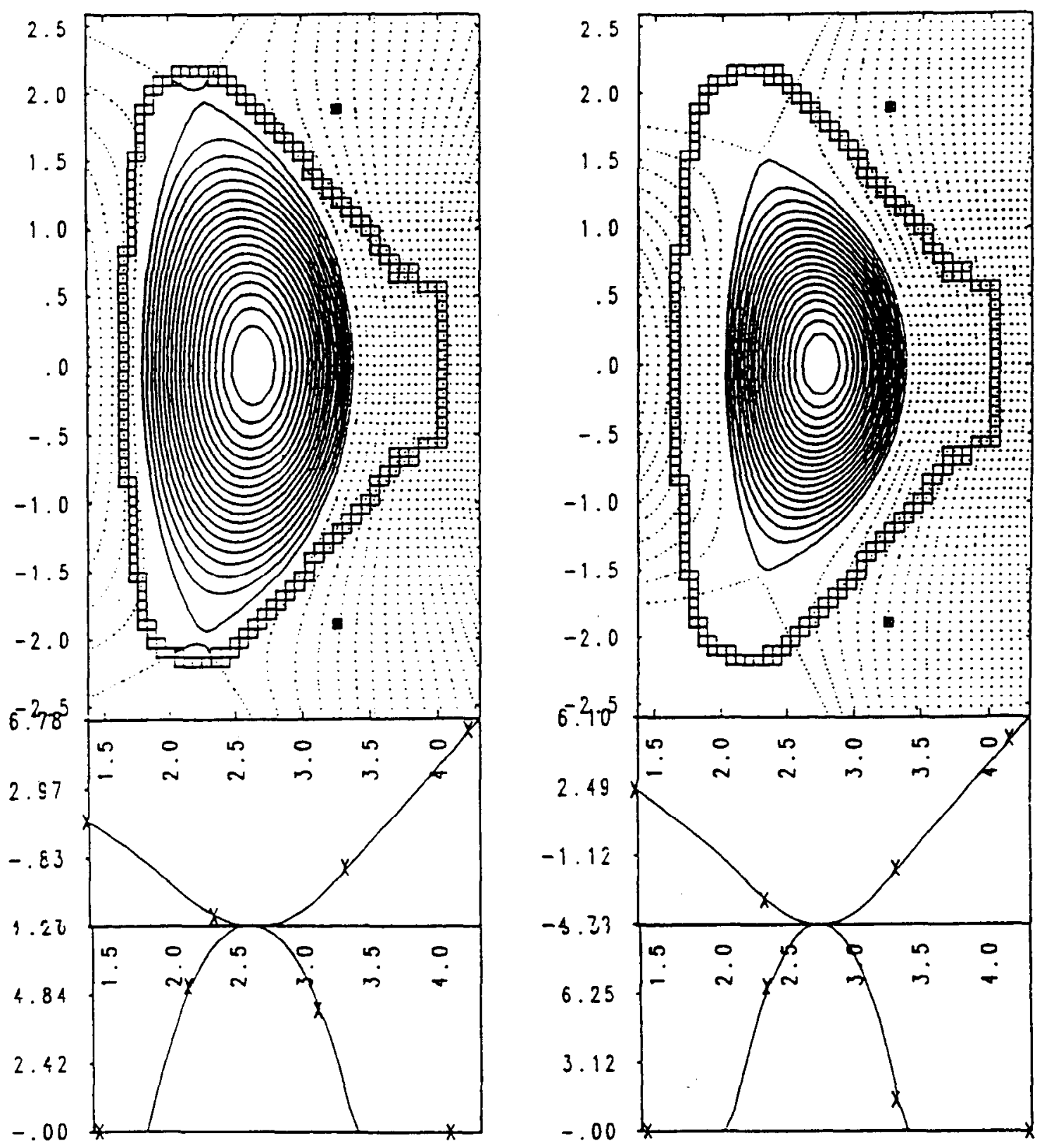

Fig. 5.17. Plasma flux surface contours inside the TSC representation of the vacuum vessel for (a) elongation $\kappa_{95}=2.2$ and (b) aspect ratio $A=4.0$ with elongation $\kappa_{95}=2.0$. 

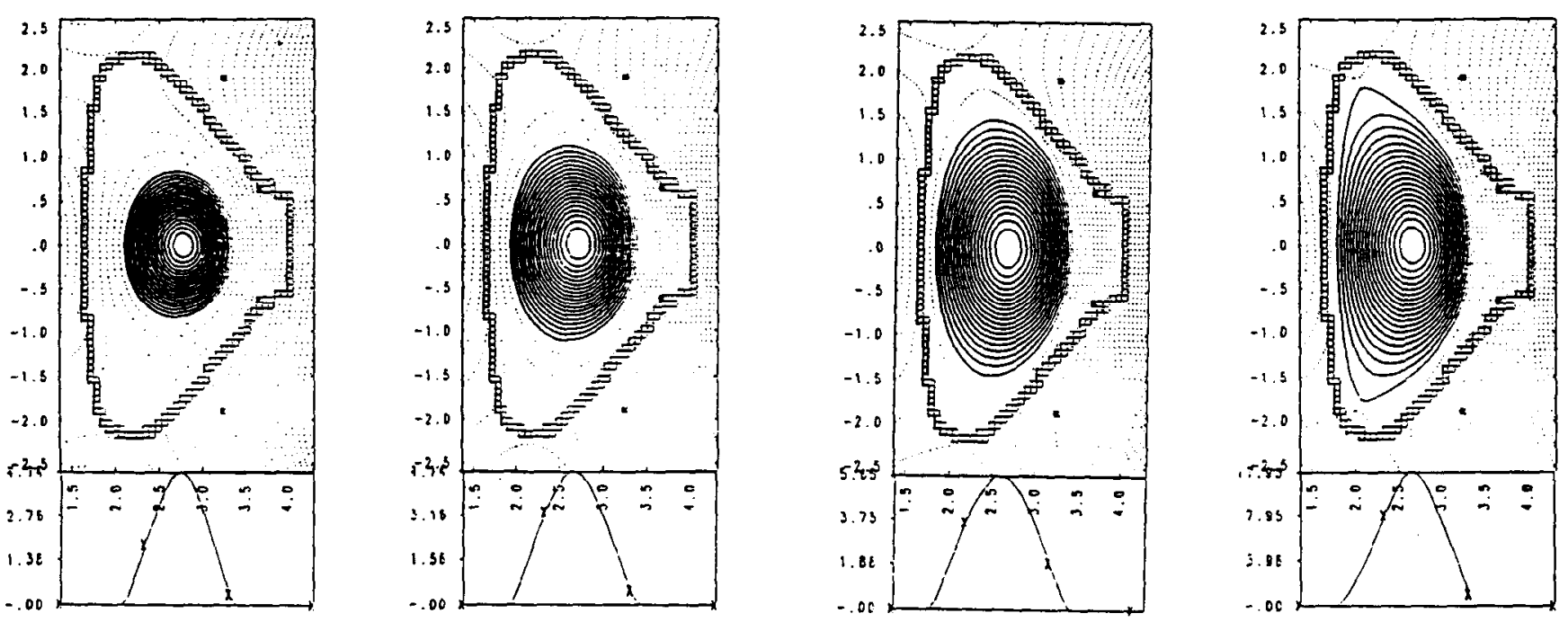

$$
\begin{aligned}
& t=1.0 \mathrm{sec} \\
& R=2.75 \mathrm{~m} \\
& a=0.64 \mathrm{~m} \\
& \kappa=1.3 \\
& I_{p}=2.0 \mathrm{MA} \\
& I_{i / 2}=0.5
\end{aligned}
$$

$$
\begin{aligned}
& t=2.0 \mathrm{sec} \\
& R=2.68 \mathrm{~m} \\
& a=0.74 \mathrm{~m} \\
& \kappa=1.5 \\
& I_{p}=3.5 \mathrm{MA} \\
& l_{j} / 2=0.5
\end{aligned}
$$

$$
\begin{aligned}
& t=3.5 \mathrm{sec} \\
& R=2.62 \mathrm{~m} \\
& a=0.80 \mathrm{~m} \\
& \kappa=1.8 \\
& I_{p}=6.0 \mathrm{MA} \\
& l_{i} / 2=0.45
\end{aligned}
$$

$$
\begin{aligned}
& t=18.5 \mathrm{sec} \\
& R=2.56 \mathrm{~m} \\
& a=0.78 \mathrm{~m} \\
& K=1.95 \\
& I_{p}=11.0 \mathrm{MA} \\
& l_{i} / 2=0.45
\end{aligned}
$$

Fig. 5.18. Plasma flux surface contours inside the TSC representation of the vacuum vessel for (a) $t=1.0$ $\mathrm{s}$ with elongation $\kappa_{95}=1.3$, (b) $t=2.0 \mathrm{~s}$ with elongation $\kappa_{95}=1.5$, and (c) $t=3.5 \mathrm{~s}$ with elongation $\kappa_{95}=1.8$ during rampup, and (d) $t=18.0 \mathrm{~s}$ with elongation $\kappa_{95}=1.95$ during rampdown. 
plasma is grown from the outside limiter. It shows that the plasma elongation must not be increased too rapidly while the plasma is growing in minor radius in order to maintain vertical stability.

Several comparisons have been made between vertical growth times calculated by TSC and by two linear MHD stability codes, LLNL and NOVAW (Ref. 11). The recently developed LLNL code was used to verify the TSC code for one configuration. The agreement was within $2 \%$, with the LLNL and TSC growth times being 20.7 and 21.0 $\mathrm{ms}$, respectively. Vertical growth times were also calculated for several BPX plasmas with differing elongations and vacuum vessel configurations, all of which agreed very well. In addition, active feedback control calculations were made with NOVA$\mathrm{W}$ for different flux-loop locations. These will be discussed in a later section, but again confirm results determined using TSC.

\section{V.E.2. Vertical Position Control}

Active feedback control is required to fully stabilize the plasma vertical position. This is accomplished using the internal control coils (IC1) located between the vacuum vessel and the TF coil. TSC is used to simulate the vertical control in order to determine current and voltage requirements for the IC1 coils. The feedback control used is a twolevel proportional-derivative-integral (PDI) classical scheme, where the difference in flux between two loops, located up-down symmetrically inside the vacuum vessel, determines a required feedback current for the IC1 coils. The difference between the required feedback current and the actual current in the IC1 coils is then used to determine a voltage that is applied to the coils. Thus, if $\Psi_{1}$ and $\Psi_{2}$ are the poloidal flux values measured at the two flux loops, the desired feedback current and requested voltage to be applied to the IC1 coils are given by

$$
I_{f e e d b a c k}=G_{p}^{I}\left(\Psi_{1}-\Psi_{2}\right)+G_{d}^{I}\left(\dot{\Psi}_{1}-\dot{\Psi}_{2}\right)
$$

and

$$
V=G_{p}^{V}\left(I_{\text {feedback }}-I_{\text {actual }}\right) .
$$

In all of the simulations, both proportional and derivative terms are used to determine the feedback current, and proportional only is used to determine the voltage. Typically, simulations are done by examining the plasma response to a step input from the control system, causing the plasma to move off the midplane to a new steady-state position.

Shown in Fig. 5.19 are the time trajectories of the plasma vertical position, and the current and voltage on the IC1 coil corrresponding to a step input request corresponding to approximately a 2 -cm displacement. This was for a standard EOB plasma configuration with $\kappa_{95}=2.0, \ell_{i} / 2=0.4$, and an unstabilized growth time of $16.5 \mathrm{~ms}$. The gains found to yield the best response are $G_{p}^{I}=1.0 \times 10^{7}$ $\mathrm{A} / \mathrm{Wb}$ and $G_{d}^{I}=1.25 \times 10^{5} \mathrm{~A}-\mathrm{s} / \mathrm{Wb}$. From the trajectories, the maximum current reached is 230 $\mathrm{kA}$-turns, and the voltage was not allowed to exceed $500 \mathrm{~V} /$ turn. In order to see the change in the behavior, various maximum voltages were used with fixed gains, and this is shown in Fig. 5.20. It is clear that a strong departure from the very high voltage case $(5000 \mathrm{~V} /$ turn $)$ takes place somewhere between 500 and $400 \mathrm{~V} /$ turn. The voltage available determines how quickly the IC1 coil can increase its current to the required feedback level, which generates the restoring radial field. It appears that $500 \mathrm{~V} /$ turn is the minimum required to adequately control the plasma vertical position for the range of parameters expected in normal operation. For this available voltage, plasmas with $\kappa_{95}=2.0$ and with unstabilized growth times longer than 12.0 ms can be controlled, corresponding to $\ell_{i} / 2$ values less than 0.45 from Fig. 5.12. (Note that the definition of $\ell_{i}$ used here differs from that used in DIII$\mathrm{D}$ by a factor of about 1.39 , so that the $12.0-\mathrm{ms}$ growth rate would correspond to $\ell_{i} / 2=0.625$ by their definition.) We have also demonstrated that plasmas with unstabilized growth times of $9.0 \mathrm{~ms}$ corresponding to $\ell_{i} / 2=0.49$ (our definition) can be controlled with a doubling of the power supply voltage to $1000 \mathrm{~V} /$ turn.

Another issue in vertical control is the time delay between receiving the flux-loop signals and activating the power supply to drive the required voltage across the IC1 coil. An example of this is shown in Fig. 5.21 for $0.0-, 2.0$, and 4.0-ms time delays. The gains are those given above, and the voltage maximum is set at $500 \mathrm{~V} /$ turns. As the time delay is increased, the plasma vertical position response becomes more oscillatory. At some value of time delay, the plasma would not be controlled. It is found that a time delay less than or equal to 3.0 ms can be controlled with the given IC1 coil current and voltage limits $\left(I_{\max } \leq 300 \mathrm{kA}\right.$-turns and $V_{\max } \leq 500 \mathrm{~V} /$ turn). If the $\mathrm{IC} 1$ coil current limit were increased to 400 to $450 \mathrm{kA}$-turns, a $4.0-\mathrm{ms}$ delay could be tolerated. To examine the effect of the voltage limit on the time delay behavior, several simulations were done for the 4.0-ms time delay and are shown in Fig. 5.21. It is clcar that the $\mathbf{5 0 0}$ $\mathrm{V} /$ turn case is beginning to depart substantially from the higher voltage cases. It requires more voltage to control when a time delay is present than when it is not. However, since the envelope inside which the oscillations decay is strongly damped, 


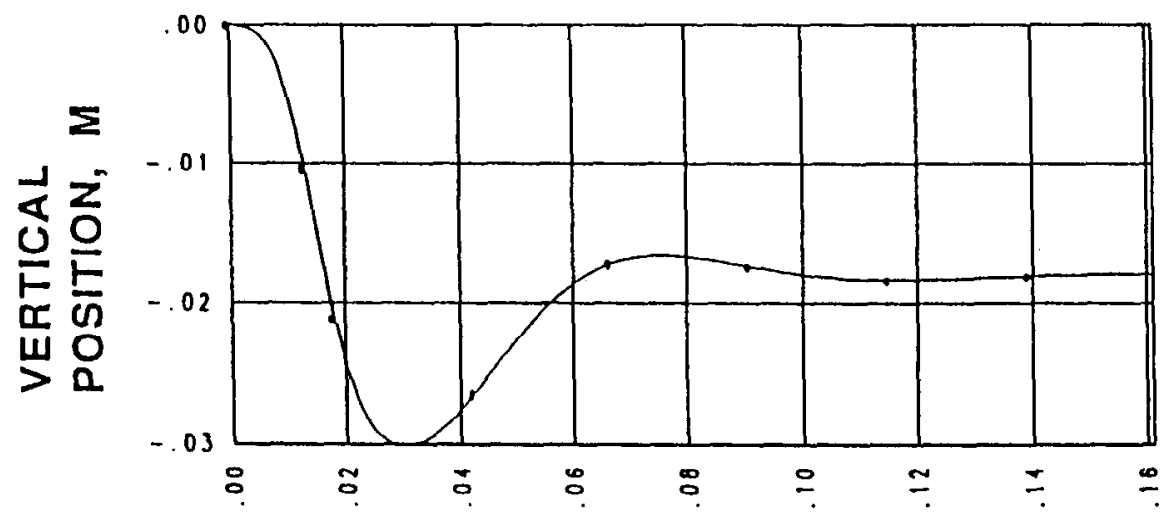

TIME, SEC

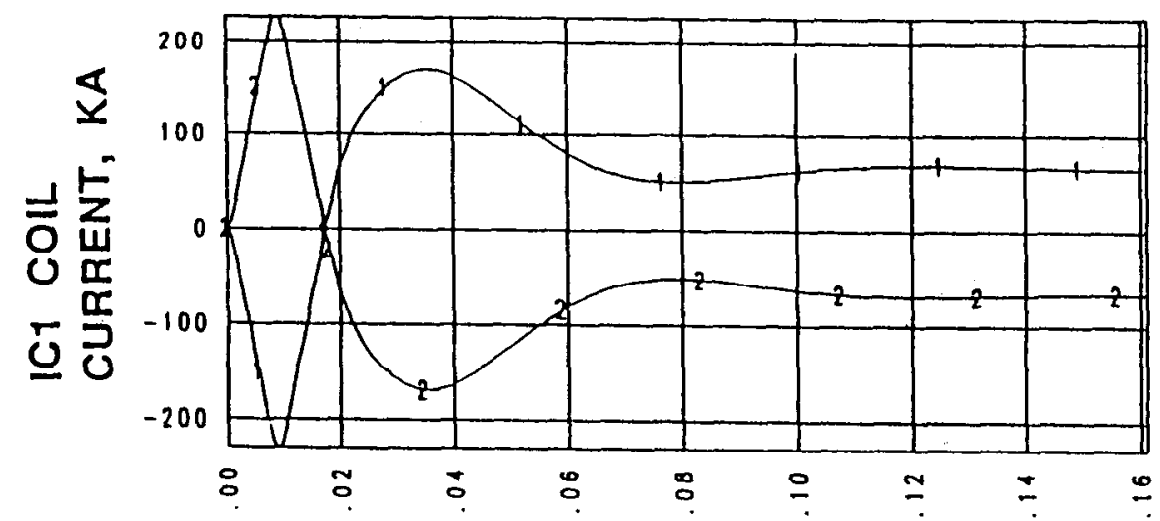

TIME, SEC

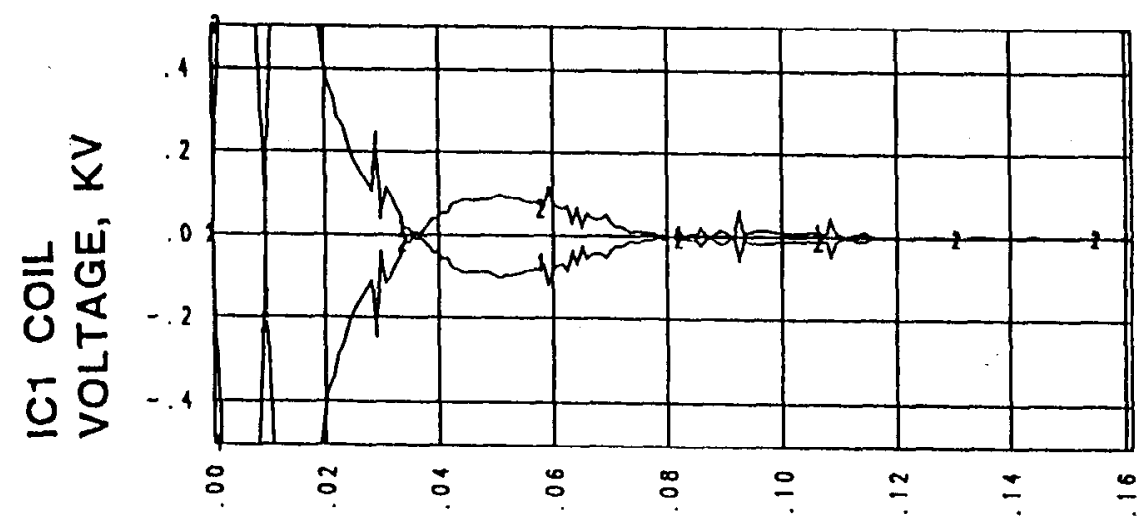

TIME, SEC

Fig. 5.19. Time trajectories of the (a) plasma vertical position, (b) current, and (c) voltage in the IC1 coils. 


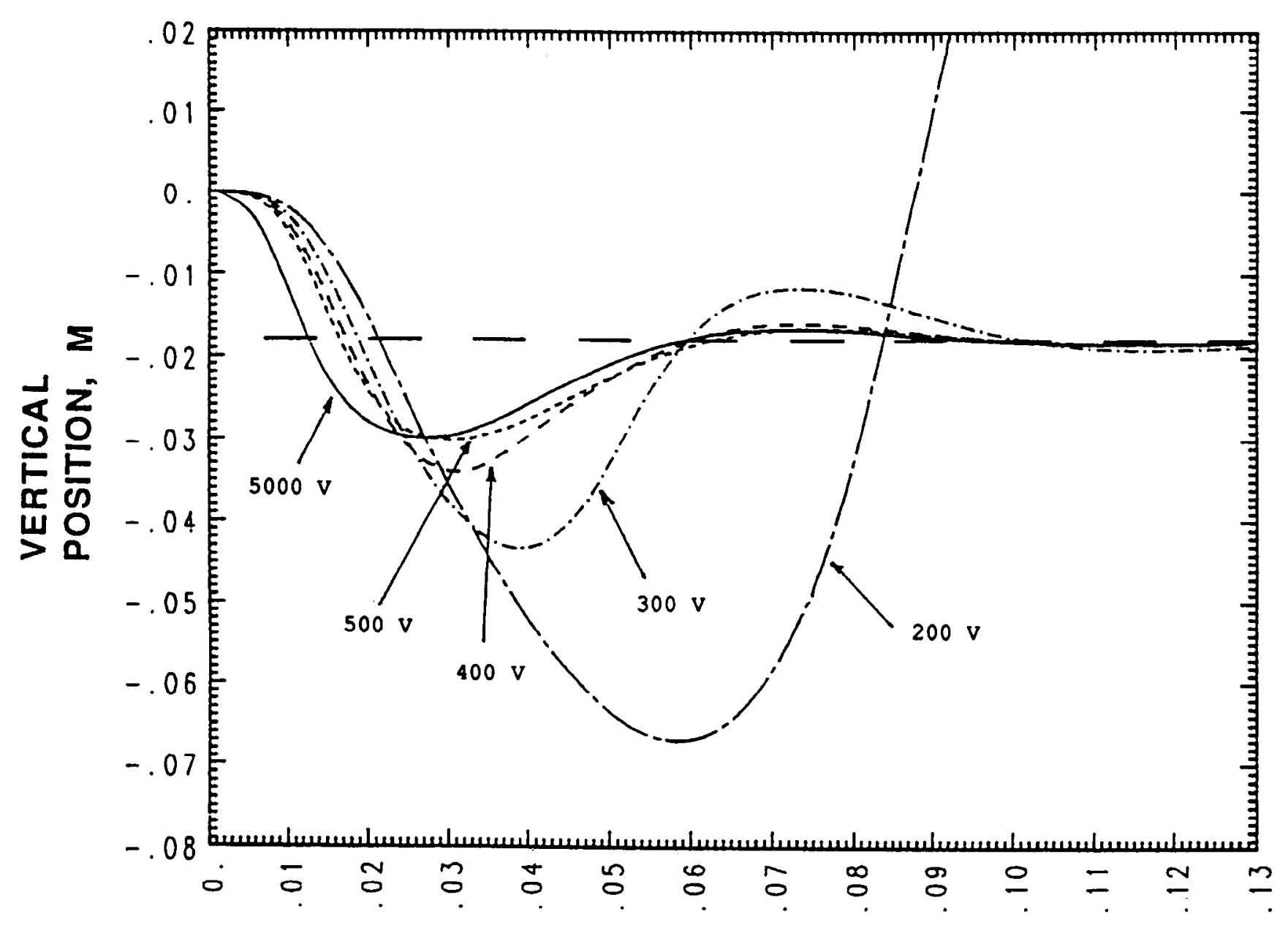

TIME, SEC

Fig. 5.20. Time trajectories of the plasma vertical position with various maximum voltages and fixed feedback gains. 
TIME DELAY VARIATION

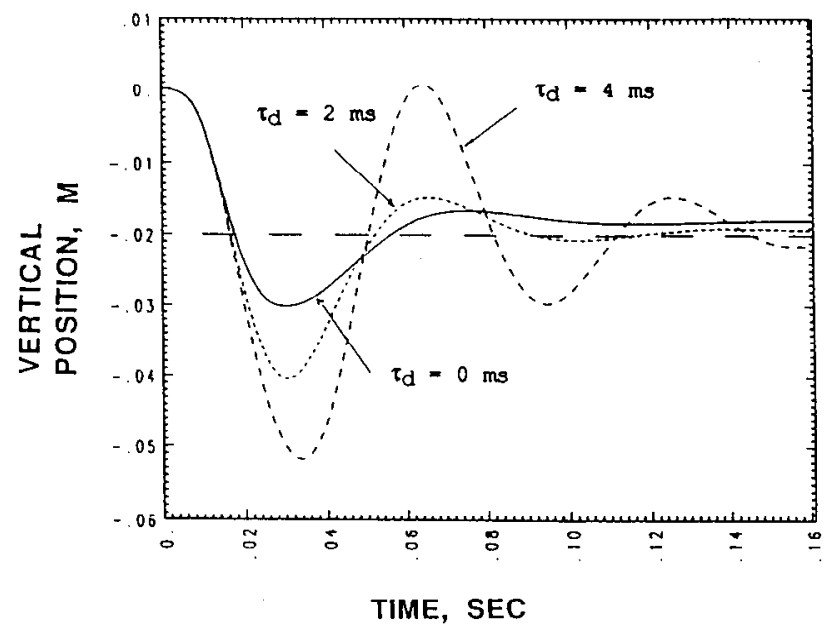

VARIATION OF MAXIMUM VOLTAGE TIME DELAY $=4.0 \mathrm{MS}$.

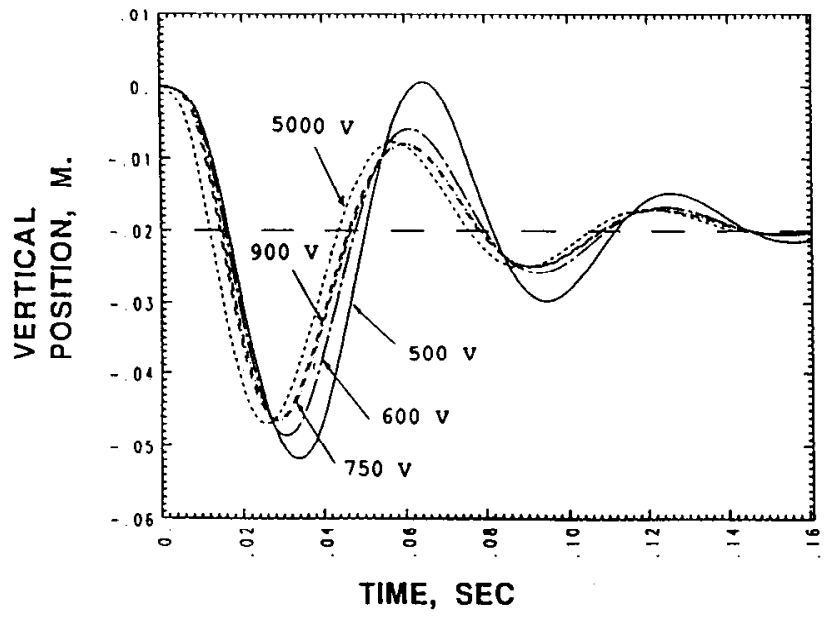

Fig. 5.21. Time trajectories of the plasma vertical position with (a) various time delays and fixed feedback gains and (b) with a time delay of $4.0 \mathrm{~ms}$ and various maximum voltages and fixed feedback gains.

the $500 \mathrm{~V} /$ turn still appears to be satisfactory.

Two special plasmas were examined for their vertical control characteristics, an elongation $\kappa_{95}=$ 2.2 and a single null, both with $\ell_{i} / 2=0.34$. The same gains were used, and they were found to be controlled satisfactorily within the same limits for current and voltage on the IC coils.

The effect of different locations for the flux loops was examined by moving them to new locations inside the vacuum vessel and attempting the same control simulations. It was found that the vertical position control is satisfactory for flux loops on the inboard vessel wall with vertical positions be- low $1.0 \mathrm{~m}$ and on the outboard vessel wall near the port entrance. These results were confirmed when a plot of the perturbed asymmetric flux was made. An example of this is given in Fig. 5.22, showing contours of constant asymmetric flux inside a previous vacuum vessel design when the plasma is displaced from the midplane. The third contour from the center touches, or is very close to, the vessel in precisely the regions found to yield the best control behavior. This contour represents the largest value of asymmetric flux which could be measured by a flux loop located on the vessel. Locating the flux loops in regions of largest asymmetric flux increases their sensitivity to vertical plasma motion. Other regions are less sensitive, requiring larger gains, or are too insensitive, not allowing satisfactory control. These results were compared to those of a similar study performed with the lincar MHD code NOVA-W (Ref. 11), and the results agreed very well.

All the above control simulations examine a 2.0$\mathrm{cm}$ offset from the midplane, representing the maximum allowable excursion that can be controlled. It is not expected that this situation will arise very often, but rather a small scale control about $Z=0$ corresponding to random perturbations will dominate the control systems functions. In order to guarantee that the IC1 coil power supply can handle this requirement, simulations were done to examine the root-mean-square current and voltage requirements for random vertical position excursions not exceeding $1.0 \mathrm{~cm}$ from the midplane. An example of a random trajectory for the vertical position, and the corresponding IC coil current and voltage, are given in Fig. 5.23. This was the result of random fluctuations with an autocorrelation time of $30 \mathrm{~ms}$ superimposed on the flux loop measurements.

In summary, we find the vertical stability and the vertical position control system in BPX to be completely acceptable, meeting all the requirements set forth in the GRD. If the IC1 coils have the capability of providing at least $\pm 300 \mathrm{kA}$-turns of current at a voltage of $500 \mathrm{~V} / \mathrm{turn}$, with a maximum effective time delay of $2 \mathrm{~ms}$, then vertical stability should be ensured for all times during all standard operating modes. Higher elongation plasmas, at least up to $\kappa_{95}=2.2$, should also be stable to axisymmetric instabilities on all time scales. However, aspect ratio 4 plasmas with elongation $\kappa_{95}=$ 2 are unstable to a rapidly growing vertical instability and thus would not be possible to produce in the present BPX design. 


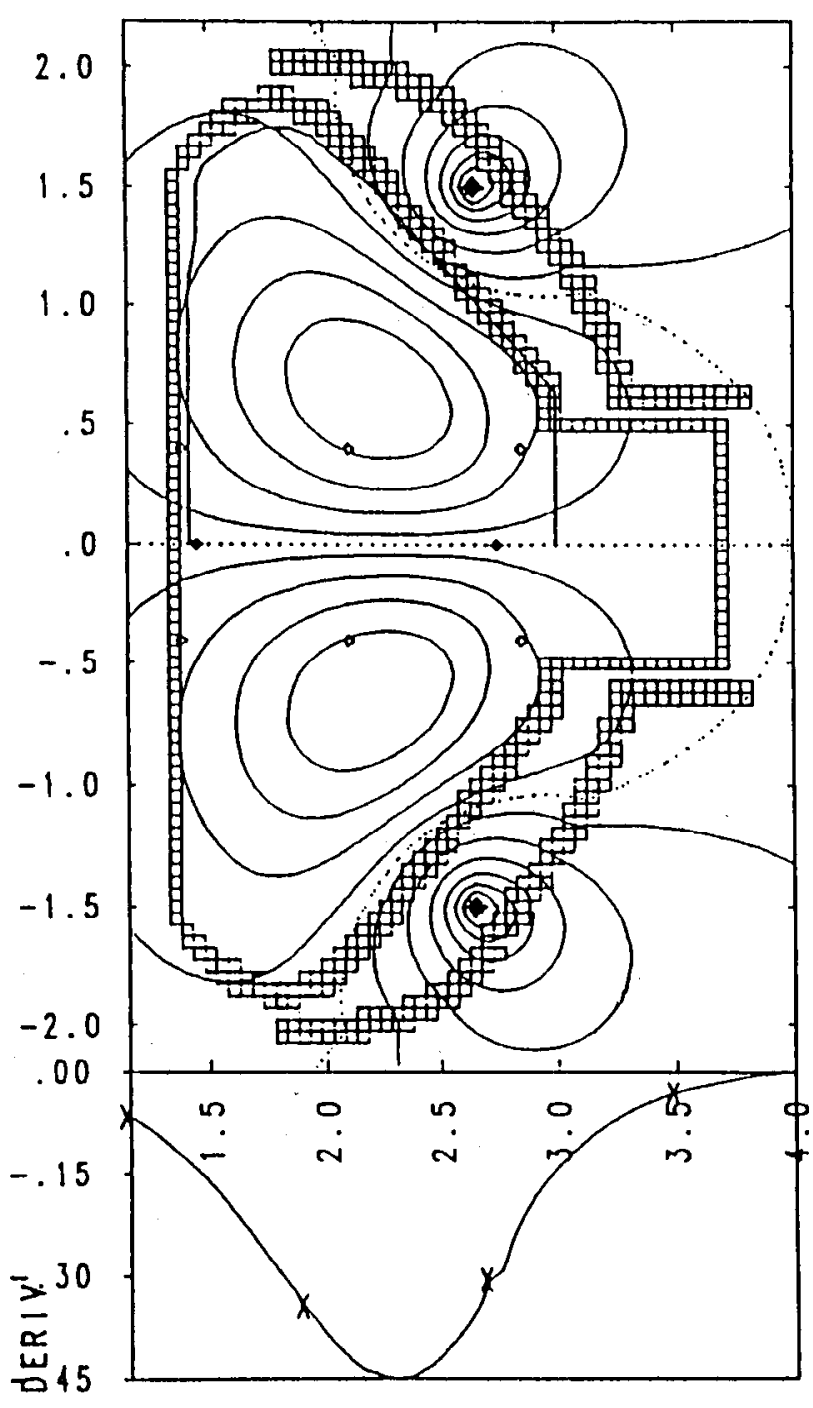

Fig. 5.22. Asymmetric flux contours inside a previous vacuum vessel design when the plasma is displaced from the midplane.

\section{V.F. RADIAL POSITION CONTROL}

Although the radial position is stable for an elongated plasma, feedback control is required to keep the plasma positioned inside the vessel, away from vessel walls, when plasma parameters deviate from nominal. The external PF coils are preprogrammed with a specific plasma equilibrium evolution in mind. However, any perturbations in the equilibrium properties will lead to plasma position and shape changes. These deviations of the plasma shape and position from the desired values are corrected with the shape control system described in Sec. V.D using the primary PF coils. However, these large relatively distant coils can only respond on time scales longer than $100 \mathrm{~ms}$. Faster time scale correction of the radial position is provided by the IC2 coils located between the vacuum vessel and the TF coil, the pair closest to the midplane. The normal function of these coils is to control the plasma boundary/antenna separation distance on fast time scales. However, the most severe requirement for radial position control is set by the specification that the system has the capability of maintaining radial control during a minor disruption, characterized by the plasma experiencing a sudden drop in $\beta_{p}$ of 0.2 . This off-normal event places stringent requirements on the IC2 coils and is simulated to verify that adequate control capability is available.

The minor disruption is initiated in the TSC simulation by artificially enhancing the transport instantaneously so that a sudden drop in $\beta_{p}$ occurs. The $\beta_{p}$ trajectory is shown in Fig. 5.24. The pressure drop leads to a rapid inward motion of the plasma. The eddy currents generated in the vessel stop the plasma from moving all the way to the inboard vessel wall. After about $10 \mathrm{~ms}$, the feedback system becomes effective and further controls the plasma. This is shown in Fig. 5.25, where time histories of the plasma geometric center, minor radius, and their difference are given for simulations with and without feedback control. With no feedback control, the plasma would drift into the inboard vessel wall. It should be emphasized that the initial inward motion is not influenced by the control system, due solely to the passive stabilization provided by the vessel, and thus is determined by its electrical resistivity and geometry. The requirement is that the plasma boundary not move inward more than $3.0 \mathrm{~cm}$, or it would impact the tiles covering the vacuum vessel. This means that both the plasma geometric center and minor radius must be monitored. Figure 5.25 shows the quantity $R_{0}-a$ with the heavy dashed line indicating the $3.0-\mathrm{cm}$ mark. The controlled case only allows the plasma to move inward $1.5 \mathrm{~cm}$ at most, while the uncontrolled case goes beyond $3.0 \mathrm{~cm}$. The IC2 coil current and voltage trajectories for the controlled case are shown in Fig. 5.26, where the maximum voltage is held at $500 \mathrm{~V} /$ turn. Proportional and integral feedback terms are used in the control scheme. Thus, if $\Psi_{1}$ and $\Psi_{2}$ are poloidal flux values inferred at two positions on the midplane that we wish to keep at the same flux value, then the desired feedback current and requested voltage to be applied to the IC2 coils are given by

$$
I_{f e e d b a c k}=G_{p}^{I}\left(\Psi_{1}-\Psi_{2}\right)+G_{i}^{I} \int\left(\Psi_{1}-\Psi_{2}\right) d t
$$

and

$$
V=G_{p}^{V}\left(I_{\text {feedback }}-I_{\text {actual }}\right)
$$


Jardin et al. MHD EQUILIBRIUM AND STABILITY

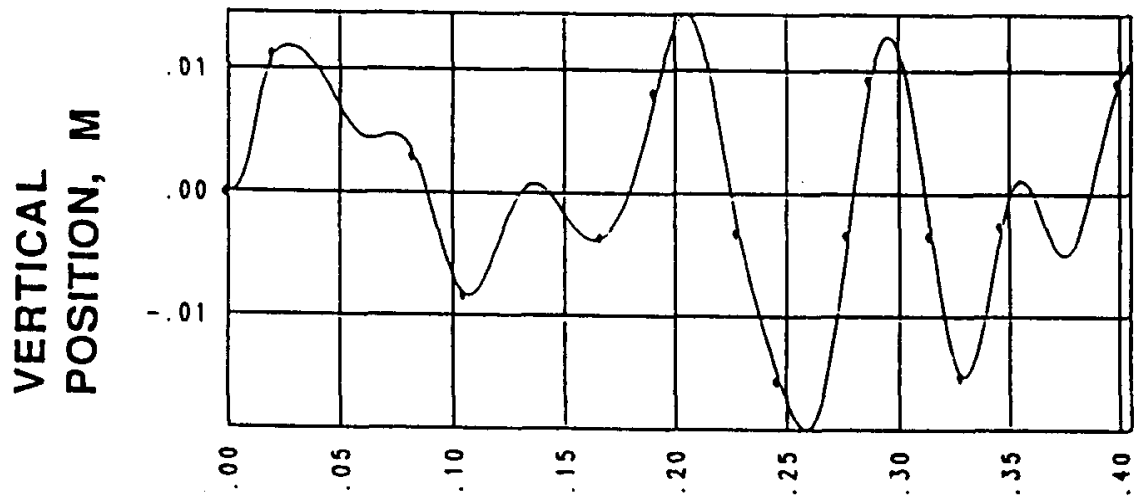

TIME, SEC

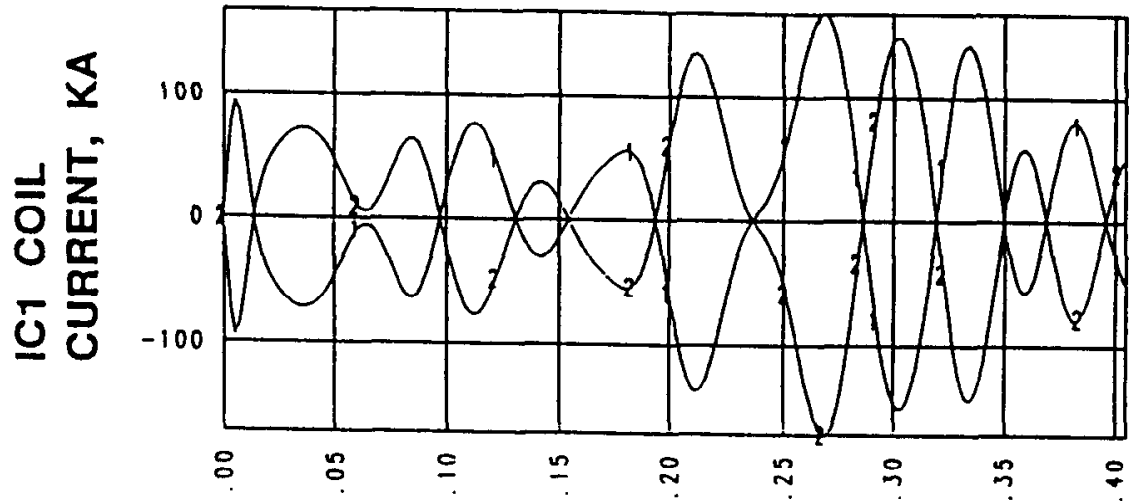

TIME, SEC

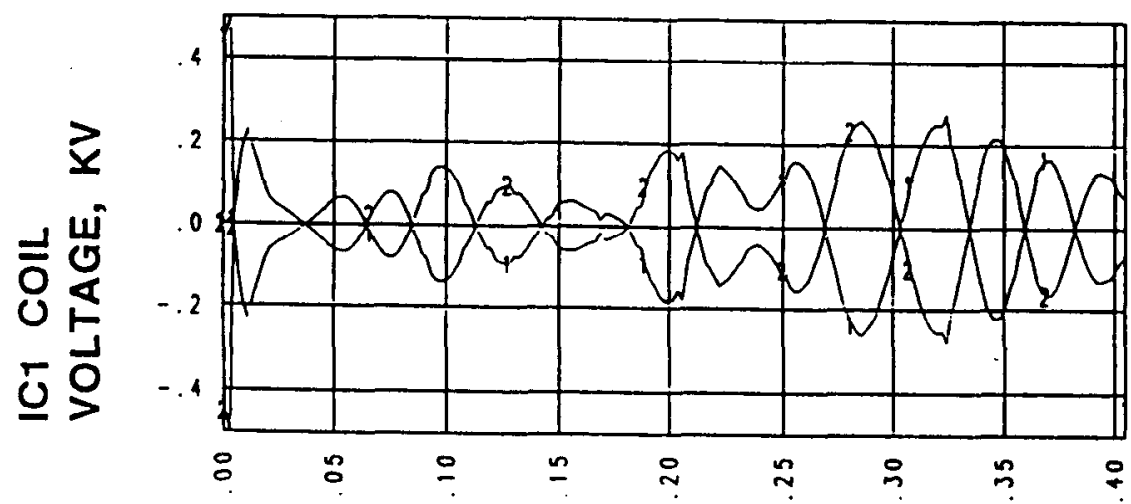

TIME, SEC

Fig. 5.23. Time trajectories of (a) plasma vertical position, (b) current, and (c) voltage in the IC coil during simulation of random signal inputs to the feedback control system. 


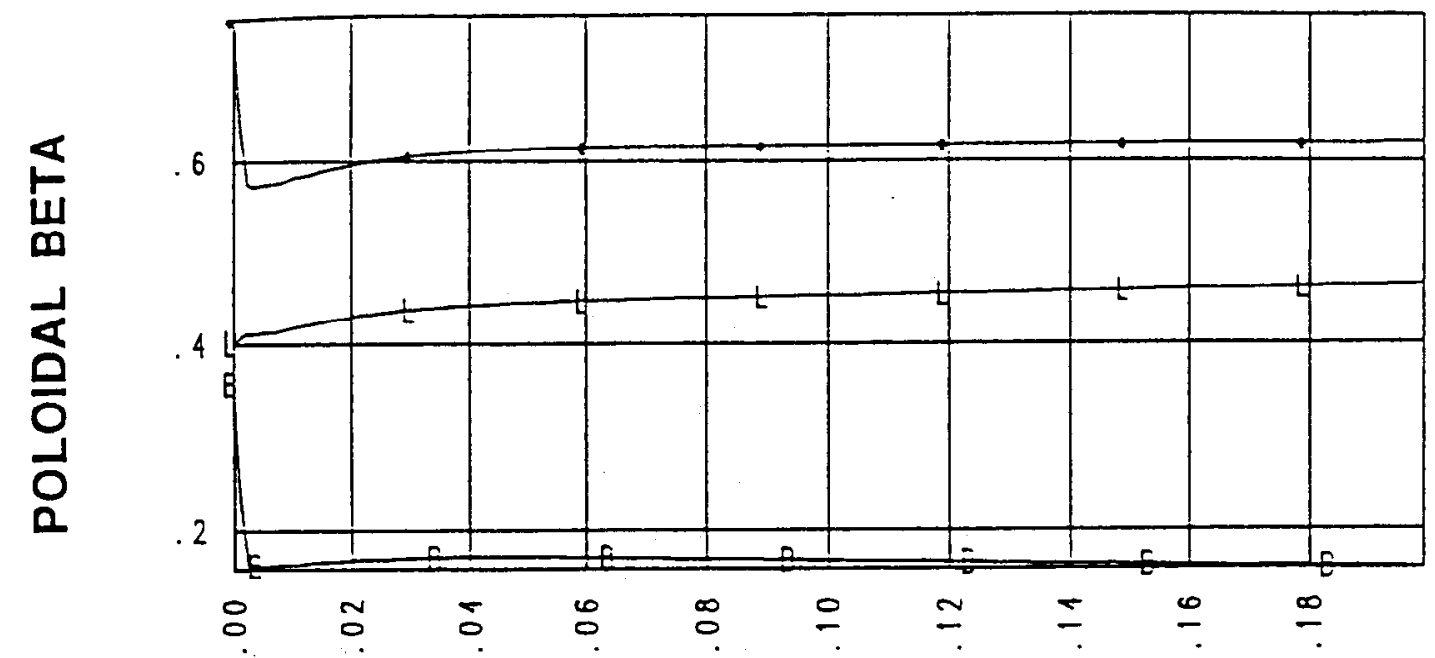

TIME, SEC

Fig. 5.24. Time trajectory of $\beta_{p}, \ell_{i} / 2$, and their sum during a minor disruption where $\beta_{p}$ drops by 0.2 .
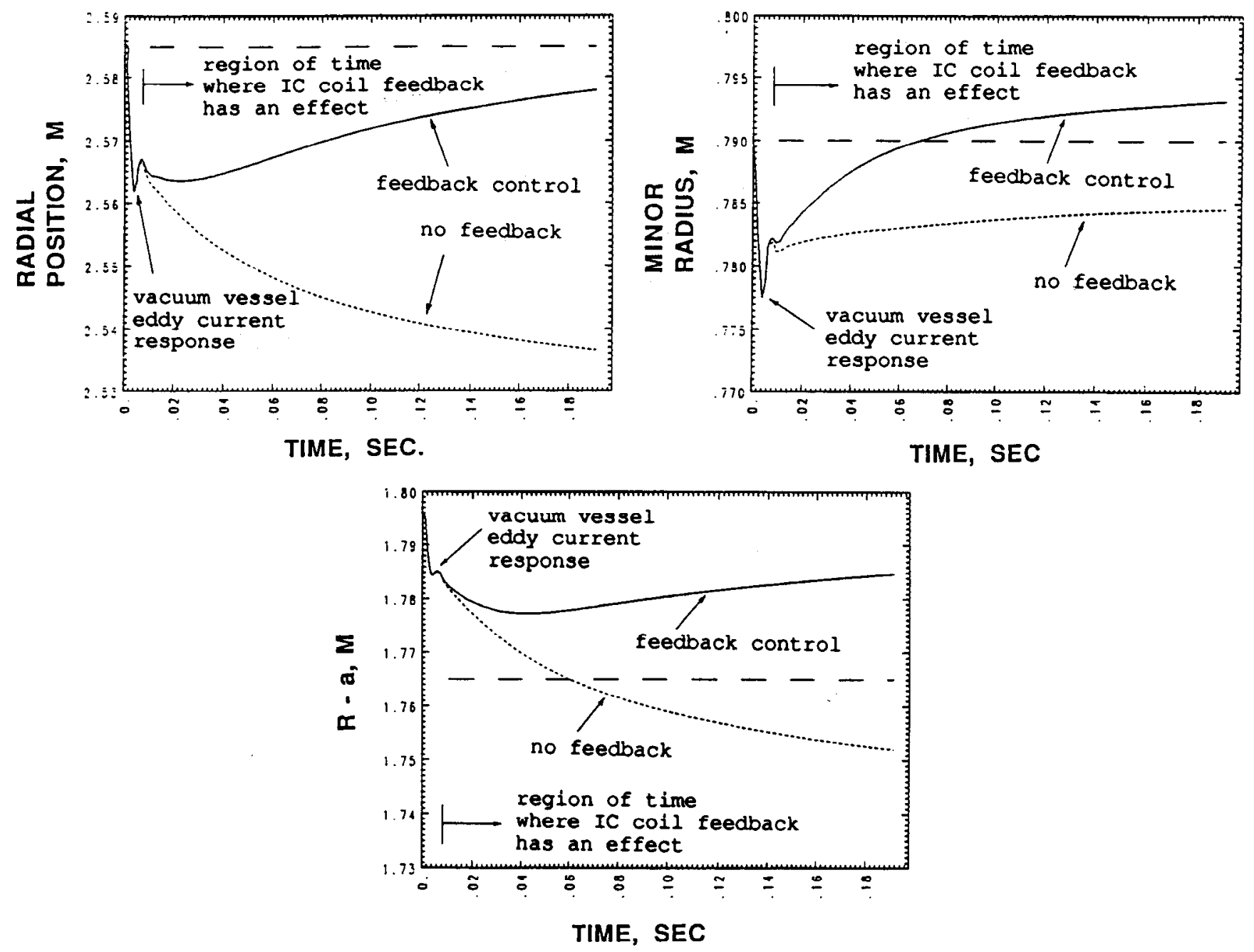

Fig. 5.25. Time trajectories of the plasma (a) geometric center $R_{o}$, (b) minor radius $a$, and (c) $R_{o}-a$ with and without feedback control. 


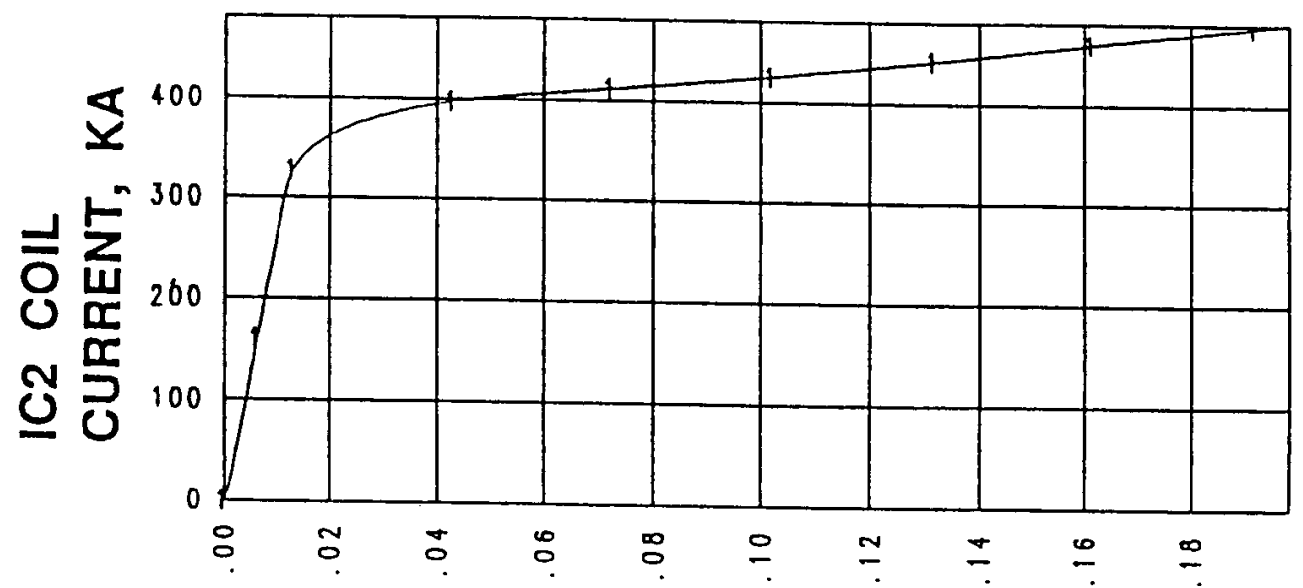

TIME, SEC

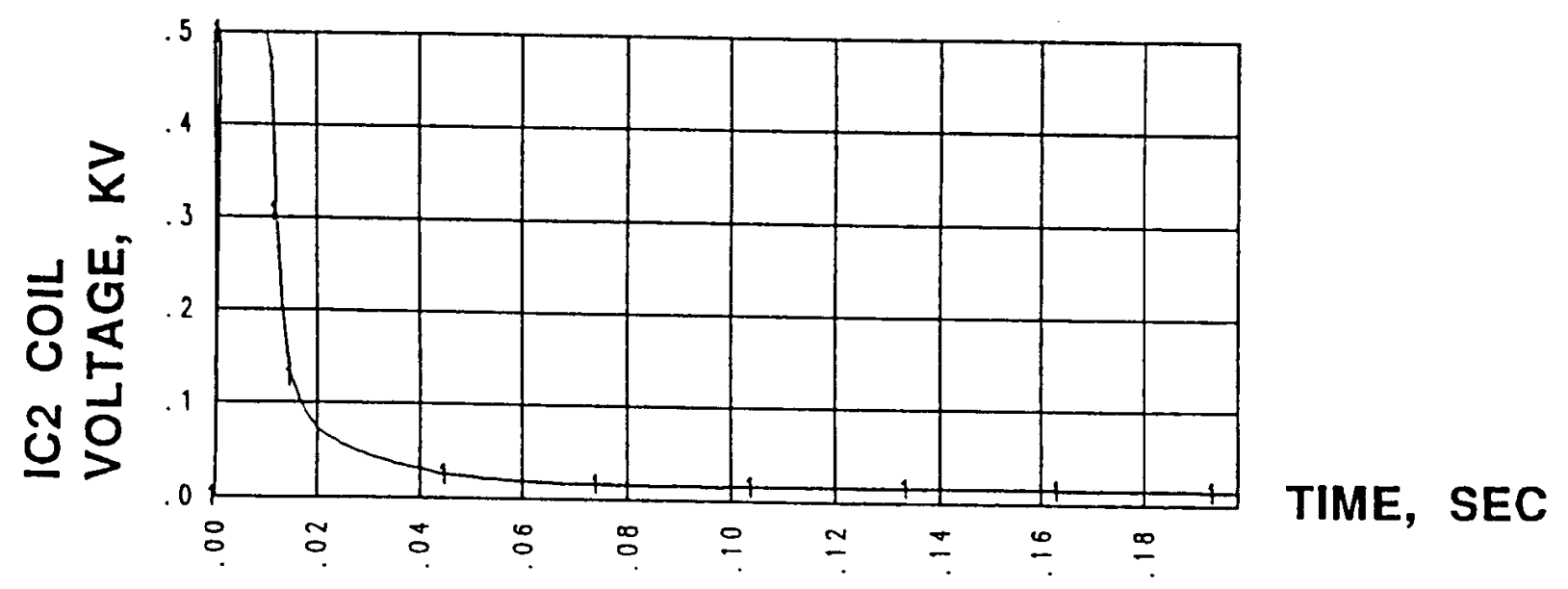

Fig. 5.26. Time trajectories of (a) current and (b) voltage in the IC coil during feedback control. 
The gains found to give satisfactory plasma response are $G_{p}^{I}=-7.5 \times 10^{5} \mathrm{~A} / \mathrm{Wb}$ and $G_{i}^{I}=$ $-6.0 \times 10^{6} \mathrm{~A} / \mathrm{Wb}-\mathrm{s}$. The flux loops are located at the nominal plasma inner and outer boundary points: $R=3.385 \mathrm{~m}, Z=0.0 \mathrm{~m}$, and $R=1.795$ $\mathrm{m}, Z=0.0 \mathrm{~m}$. In practice, the flux loops would be on the vessel walls, and magnetic probes would be used in conjunction to extrapolate the flux to the plasma boundary.

The effect of varying the maximum voltage on the plasma geometric center and minor radius trajectories is shown in Fig. 5.27. This indicates that the trajectories begin to strongly deviate from the high-voltage cases when the maximum voltage is less than $500 \mathrm{~V} /$ turn.

The IC2 coil current, shown in Fig. 5.26, tends to steady-state values exceeding $400 \mathrm{kA}$-turns. The drop in $\beta_{p}$ requires that the equilibrium vertical field be reduced to keep the plasma at the same radial position. Steady-state currents are required in the IC2 coils to achieve this. However, it is not desirable to have large standing currents in the IC2 coils because this will hinder their ability to respond to subsequent fast time scale radial motion. It is therefore desirable to transfer these steady-state currents to the outer PF coils. This leads naturally to a two-time-scale control strategy, where initially the IC2 coils react to keep the plasma radially positioned during a minor disruption, while an outer PF coil, say PF7, responds in concert on a longer time scale to eliminate any cumulative current in the IC2 coils. Thus, steadystate vertical field changes are transferred to the outer PF coils. We have demonstrated this twotime-scale control in a TSC simulation of a minor disruption where the IC2 coil feedback is identical to that of Eq. (5.11) but in addition there is a desired feedback current added to PF7 proportional to the time integral of the flux difference $\left(\Psi_{1}-\Psi_{2}\right)$. Shown in Figs. 5.28, 5.29, and 5.30 are the plasma geometric center and minor radius, IC2 coil current and voltage, and PF7 coil current and voltage, respectively, for this simulation. It can be seen in Fig. 5.29 that the IC2 coil current only reaches about $250 \mathrm{kA}$-turns and then decays toward zero, and in Fig. 5.30, a permanent change in the PF7 coil current has taken place.

In summary, we find that the GRD requirement that the device be equipped with a radial control feedback system such that an instantaneous change of $\beta_{p}$ of -0.2 results in a radial position change $\Delta\left(R_{0}-a\right)$ of less than $3 \mathrm{~cm}$ should be satisfied if the IC2 coils are capable of currents of $480 \mathrm{kA}$ turns with driving voltages of $500 \mathrm{~V} / \mathrm{turn}$. The two-time-scale feedback system in which PF7 takes over the steady-state feedback response has also been shown to work in simulations. We therefore expect the radial feedback system described here to meet all the radial control requirements set forth in the GRD, and thus the system should be able to maintain the plasma boundary/antenna separation distance on all time scales, as well as maintain plasma position during a minor disruption.

\section{V.G. FDUCIAL DISCHARGE SIMULATIONS}

The Tokamak Simulation Code ${ }^{2}$ (TSC) is used to simulate the current rampup, flattop, and rampdown phases of nominal BPX discharges. The simulations presented in this section are (a) a $P_{f u s}=$ $500 \mathrm{MW}$ DN divertor simulation, (b) a $P_{\text {fus }}=100$ MW DN divertor simulation, and (c) a $P_{f u s}=500$ MW limiter simulation. The DN divertor configuration is the primary BPX operating mode. The discharge simulations are used to predict the evolution of coil currents and divertor heat loads for engineering design analysis. The 500-MW DN case, in particular, is used to generate a detailed divertor sweep scenario accompanied by self-consistent heat loads for purposes of evaluating divertor performance. The 100-MW DN case is used to determine the volt-second requirements for the PF coil design.

TSC includes a two-dimensional transport description of the plasma, using neoclassical resistivity and the Tang-Coppi two-regime anomalous thermal conductivity model ${ }^{13}$ with $\chi_{i}=\chi_{e}$. The temperature profiles $T_{e, i}(\psi, t)$ are evolved selfconsistently in time using the transport model; however, particle transport is not treated selfconsistently. Instead, the density profile is fixed in shape for all time while the central density is given a prescribed time dependence. No attempt is made in TSC to simulate the detailed dynamics of sawteeth. Instead, the effect of sawteeth on the temperature evolution is taken into account, in the average sense, by flattening the resistivity profile inside the sawtooth inversion radius according to the prescription

$$
\eta_{\|}=\eta_{N C} \text { for } q \geq 1
$$

and

$$
\eta_{\|}=0.5 \eta_{N C}+0.5 \eta_{q=1} \text { for } q<1 .
$$

Considerable effort has been spent in calibrating the transport model used by TSC. A detailed comparision of simulation results with TFTR experimental results is presented in Ref. 16. The simulations adjust the central density and density profile shape exponent to match the experimental line-average density, use the experimen- 
Jardin et al. MHD EQUILIBRIUM AND STABILITY

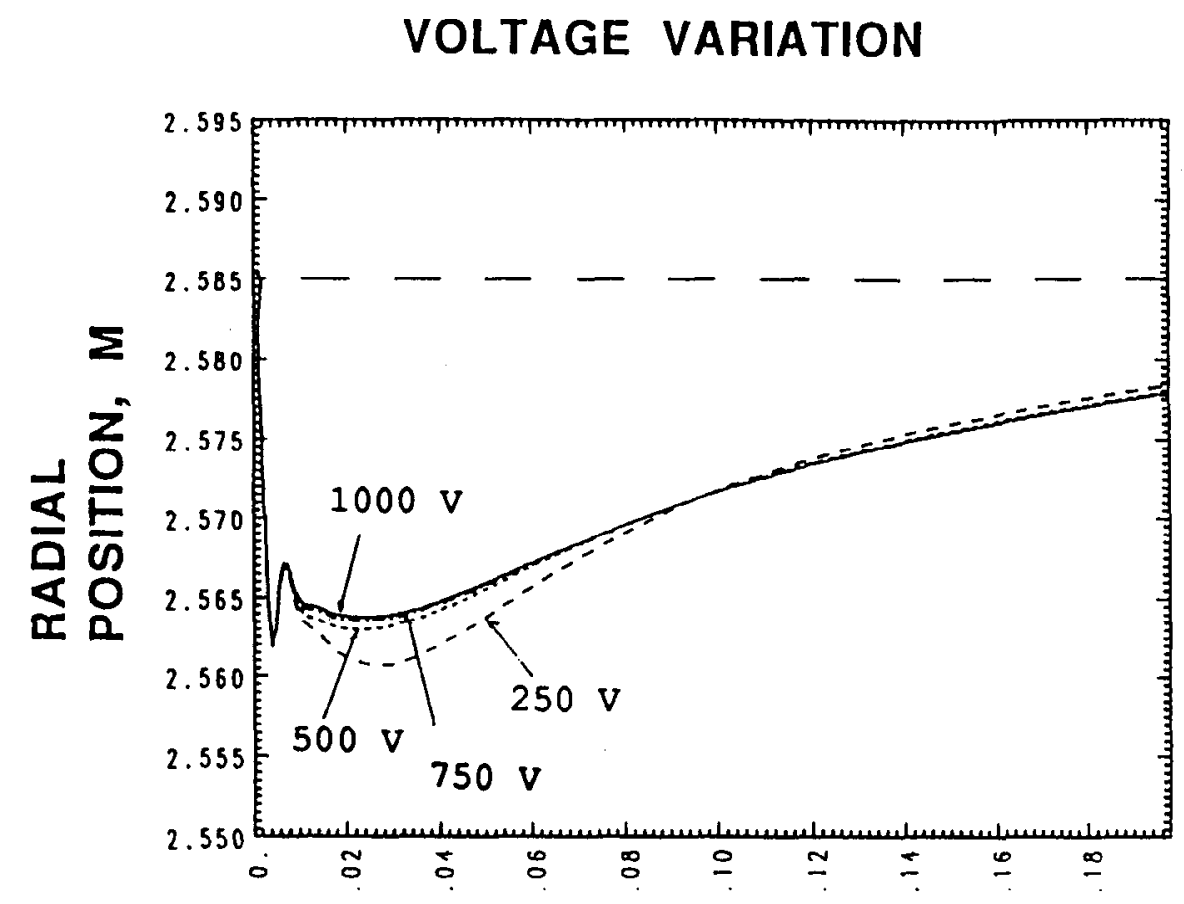

TIME, SEC

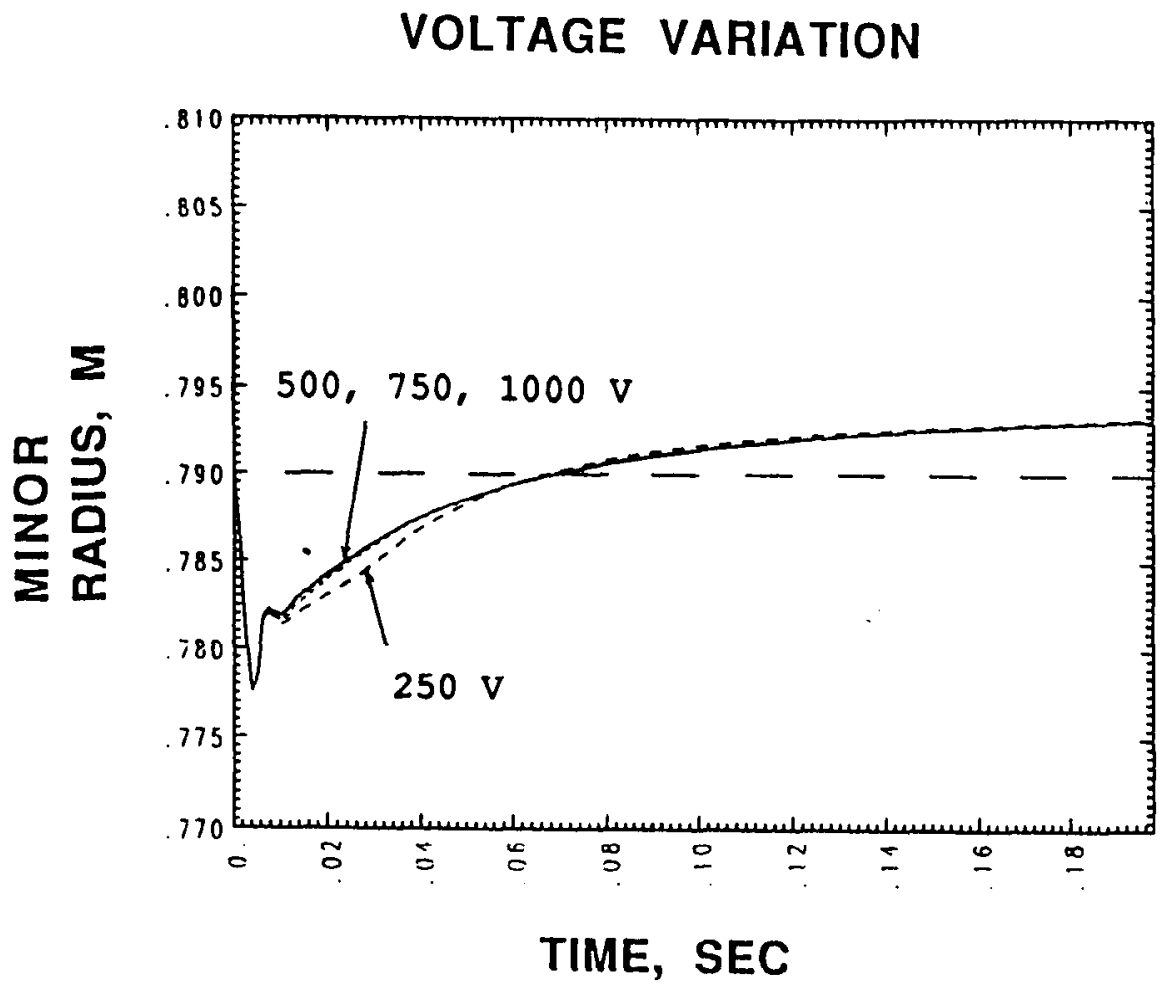

Fig. 5.27. Time trajectories of the plasma (a) geometric center $R_{o}$ and (b) minor radius a for several maximum voltages. 


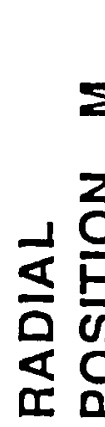

$\sum^{2} 2$

2.585

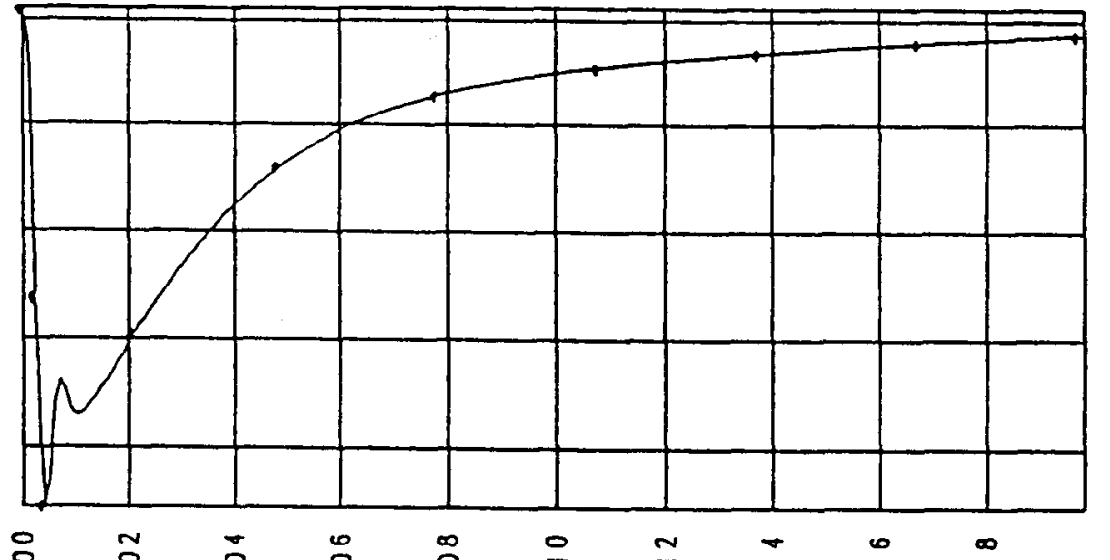

TIME, SEC

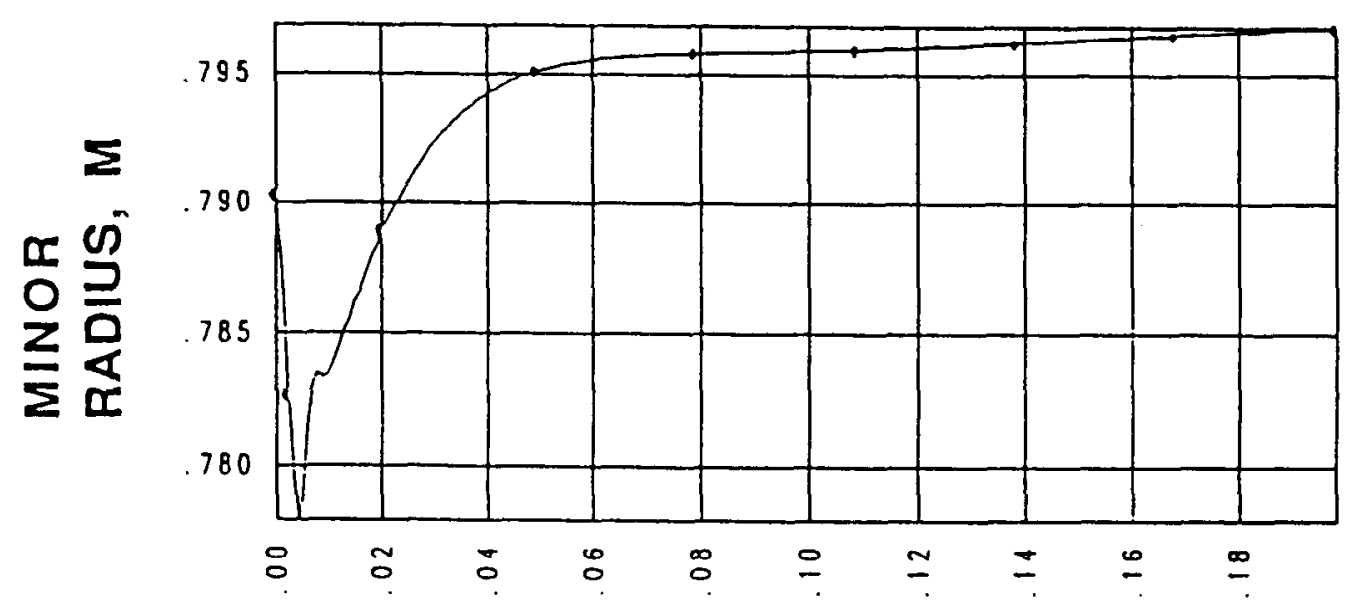

TIME, SEC

Fig. 5.28. Time trajectories of the plasma (a) geometric center $R_{0}$ and (b) minor radius a during two-time-scale radial control using IC2 and PF7. 


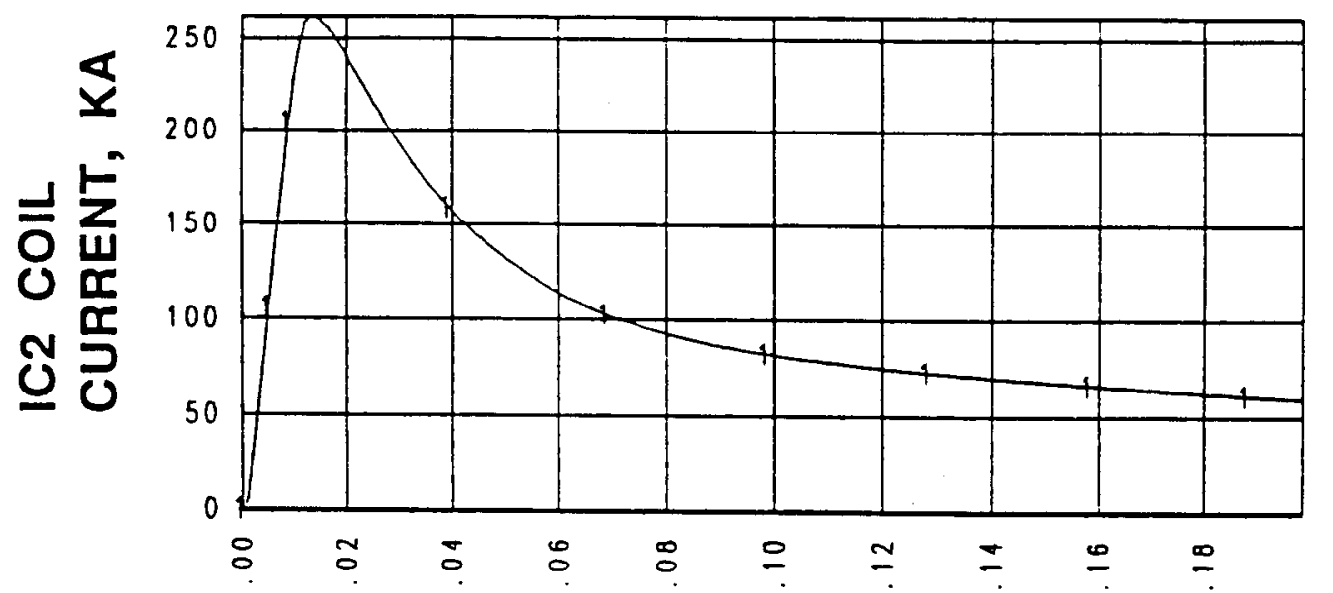

TIME, SEC

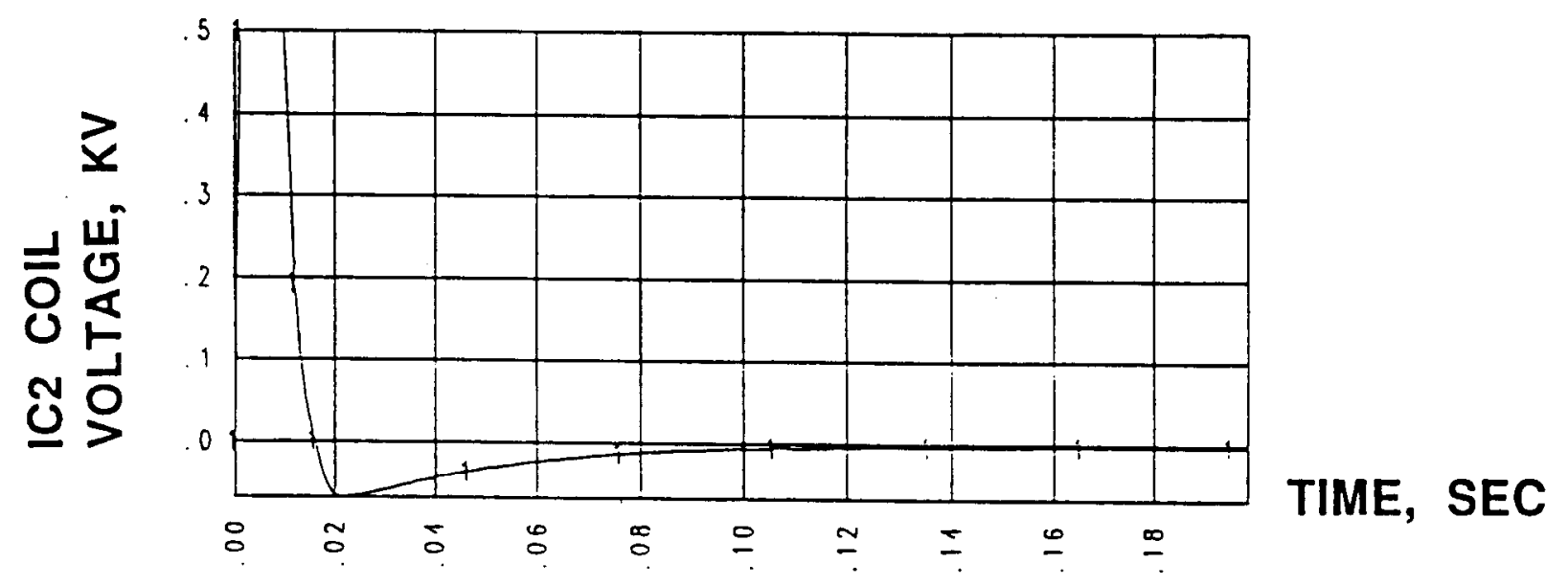

Fig. 5.29. Time trajectories of (a) current and (b) voltage on the IC2 coil during two-time-scale control using IC2 and PF7. 


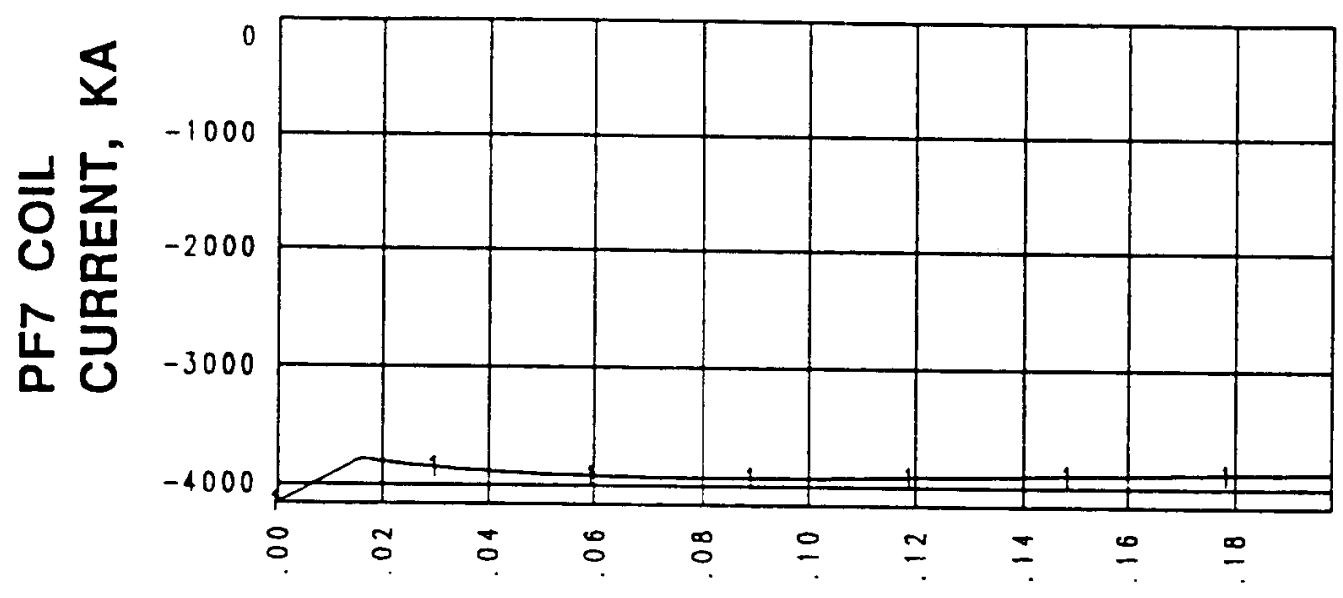

TIME, SEC

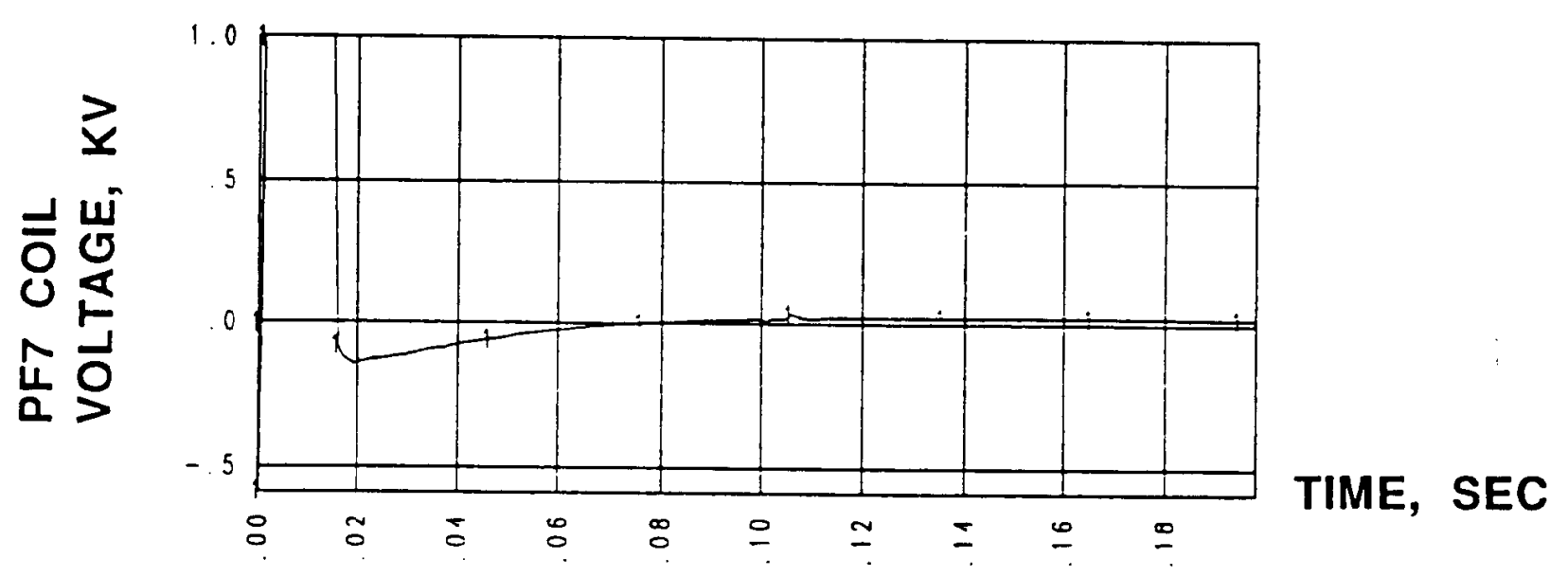
Fig. 5.30. Time trajectories of (a) current and (b) voltage on PF7 during two-time-scale control using
IC2 and PF7. 
Table 5.18. The Error of a TSC Simulation in Reproducing the Experimental Volt-Second Consumption is Shown by the Value of $\Delta \Phi_{s}^{P F}$ for Various TFTR Ohmic Shots

\begin{tabular}{|c|c|c|ccc|cccc|}
\hline & $(\mathrm{MA})$ & $\left(10^{14}\right) \mathrm{m}^{-3}$ & \multicolumn{3}{|c|}{ (Volt-seconds) } & \multicolumn{4}{c|}{ Max (keV) } \\
Shot & $I_{p}{ }^{\max }$ & $n_{e}(0)$ & TOT & RES & $\Delta \Phi_{s}{ }^{P F}$ & $T_{e}(0)^{\operatorname{Exp}}$ & $T_{e}(0)^{\operatorname{Sim}}$ & $T_{i}(0)^{E x p}$ & $T_{i}(0)^{\text {Sim }}$ \\
\hline 24088 & 2.2 & 0.35 & 16.2 & 3.4 & +0.01 & 4.4 & 4.4 & 2.9 & 3.4 \\
24095 & 2.2 & 0.35 & 16.1 & 3.6 & -0.10 & 4.4 & 4.5 & 3.0 & 2.9 \\
24096 & 2.2 & 0.65 & 16.4 & 3.4 & -0.12 & 3.8 & 3.5 & 3.0 & 3.0 \\
24098 & 1.8 & 0.30 & 14.2 & 3.4 & +0.06 & 4.5 & 4.2 & 3.1 & 3.1 \\
24100 & 1.8 & 0.70 & 14.2 & 3.3 & -0.33 & 4.0 & 3.6 & 3.2 & 2.9 \\
24093 & 1.4 & 0.37 & 12.3 & 3.2 & +0.45 & 3.4 & 3.4 & 2.7 & 2.7 \\
24089 & 1.0 & 0.66 & 11.3 & 3.4 & -0.20 & 2.5 & 2.8 & 2.2 & 2.3 \\
\hline
\end{tabular}

tally inferred value of $Z_{\text {eff }}(t)$, and input the actual (i.e., measured) time-dependent coil currents in TFTR. The coil currents are the ohmic heating current, the equilibrium field current, the variable curvature field current, and the toroidal field current. In addition to these experimental currents, a plasma current feedback control system was used in the simulation in which a fictitious loop voltage was added to the computational boundary at each time step to force the simulation plasma current to match the experimental current. The time integral of this voltage, $\Delta \Phi_{s}{ }^{P F}$ listed in Table 5.18, represents the error of the simulation in reproducing the experimental volt-second consumption.

As one example of a TFTR simulation, we show a comparison between simulation results and experimental data for ohmic TFTR discharge shot 24095. Figure 5.31 shows the evolution of plasma current and major and minor radius. Figure 5.32 shows the piecewise linear approximation to $Z_{\text {eff }}(t)$ used in the simulation together with the experimentally inferred $Z_{\text {eff }}$. Also shown is the evolution of the density profile. Here, the line-average density is chosen to match the experimental line-average while the profile shape was continuously adjusted to match the peak-to-average values. The fact that the central density value in TSC does not match the experimental central value is an indication that the actual density profile shape does not exactly match the simple profile form factor assumed in TSC. Figures 5.33 and 5.34 compare the calculated electron and ion temperatures with the experimental values. Excellent agreement is apparent not only in the central values but in the peak-to-average ratios as well. It is remarkable that the transport model is able to reproduce the entire time dependence of the profiles without adjustment. Finally, Fig. 5.35 compares the simulation and experimental values of the sawtooth inversion radius. Once again, ex- cellent agreement is found. A summary table of results from simulating seven TFTR shots is presented in Table 5.18. The transport model used in TSC is seen to reproduce essential features of the experiment. This provides some confidence to the predictions of the BPX discharge simulations described below.

\section{V.G.1. $P_{f u s}=500 \mathrm{MW}$ Divertor}

The DN divertor configuration is the primary BPX operating mode. The discharge simulations described here are used to predict the evolution of coil currents and divertor heat loads for engineering design analysis. The 500-MW case, in particular, is used to generate a detailed divertor sweep accompanied by self-consistent heat loads for purposes of evaluating the divertor energy handling performance. The evolution of plasma current and toroidal field are shown in Fig. 5.36. The current is ramped linearly in time from the initial value $I_{p}=100 \mathrm{kA}$ at time $t=0.0 \mathrm{~s}$ to the flattop value of $I_{p}=11.8 \mathrm{MA}$ at $t=7.5 \mathrm{~s}$. During this interval, the toroidal field is increased in time from $B_{t}=7.4$ $\mathrm{T}$ to $B_{t}=9.0 \mathrm{~T}$. The current and toroidal field flattop period extends until $t=13.2 \mathrm{~s}$, when the divertor has experienced at least $95 \%$ of the peak heat flux for $3.0 \mathrm{~s}$. The plasma current rampdown is piecewise linear in two stages: First the current is decreased at $1.6 \mathrm{MA} / \mathrm{s}$ for $4.5 \mathrm{~s}$. Finally, the plasma current is decreased at a rate of $0.8 \mathrm{MA} / \mathrm{s}$ to a final value of $100 \mathrm{kA}$ at $t=23.7 \mathrm{~s}$. During the plasma current rampdown, the toroidal field is decreased exponentially in time, with a time constant of $11.0 \mathrm{~s}$.

Figure 5.37 shows snapshots of the plasma/vacuum interface at various times during the plasma evolution. The plasma is grown from an outboard limited circular plasma at $t=0.0 \mathrm{~s}$, becomes diverted at $t=4.0 \mathrm{~s}$, and achieves a maximum 


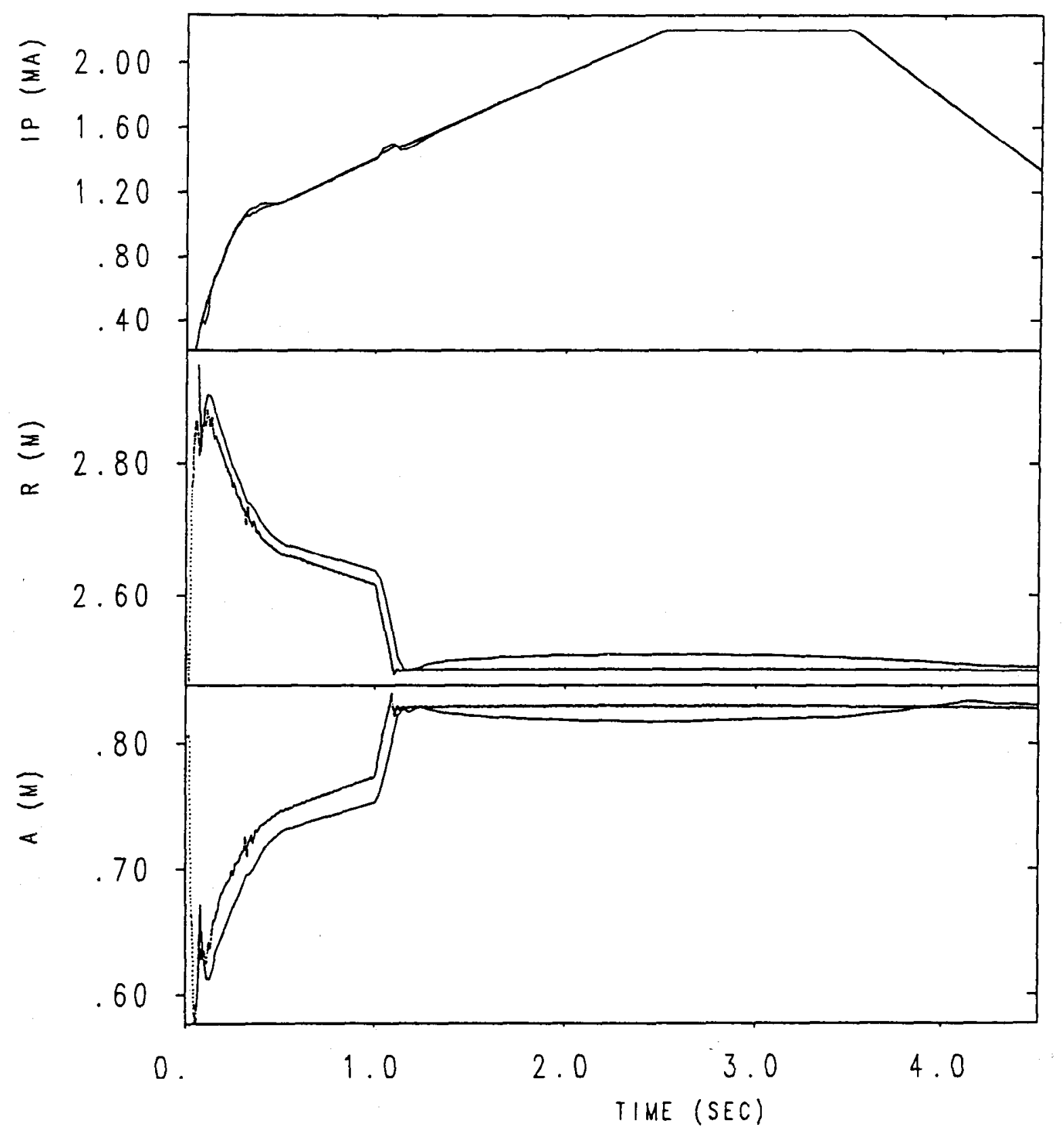

Fig. 5.31. Evolution of plasma current $\mathrm{I}_{p}$, major radius $R$, and minor radius $a$ for TFTR shot 24095 . Experimental and simulation curves are shown. 


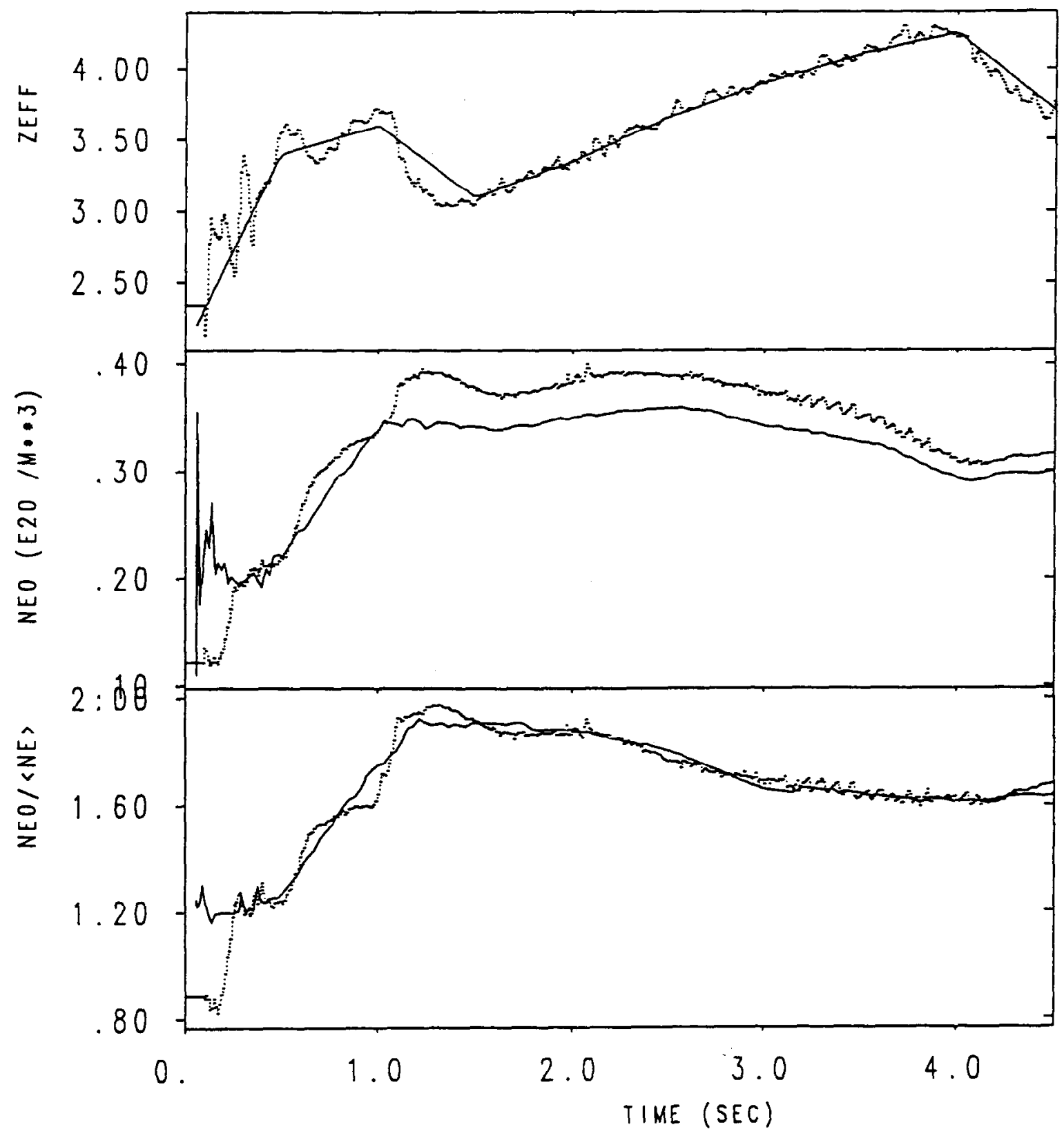

Fig. 5.32. Experiment and simulation values of $Z_{\text {eff }}$, central electron density $n_{e}(0)$, and peak-to-average electron density $n_{e}(0) /\left(n_{e}\right\rangle$ as a function of time. 


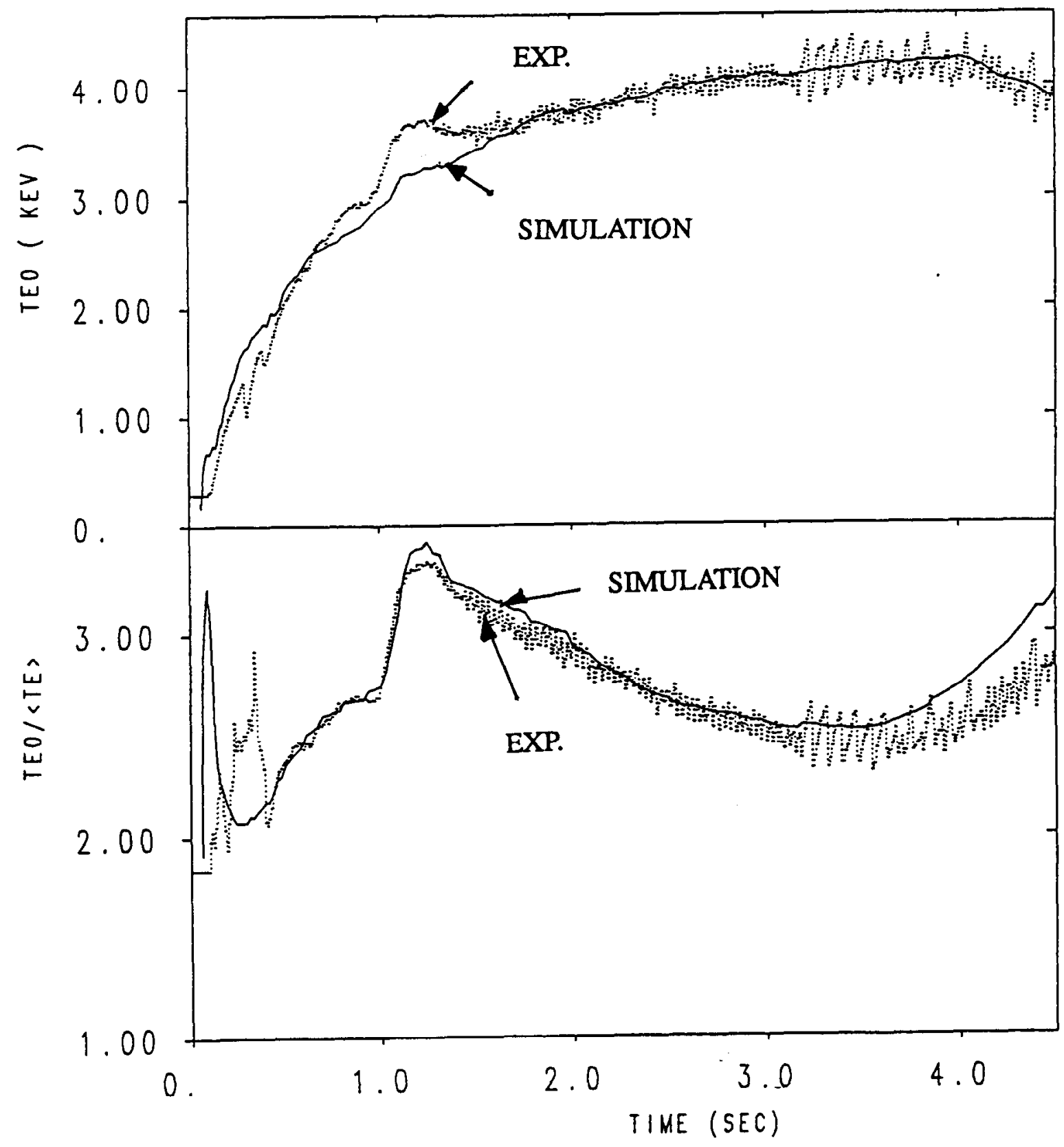

Fig. 5.33. Experiment and simulation values of central electron temperature $T_{e}(0)$, and peak-to-average electron temperature $T_{e}(0) /\left\langle T_{e}\right\rangle$ as a function of time. 


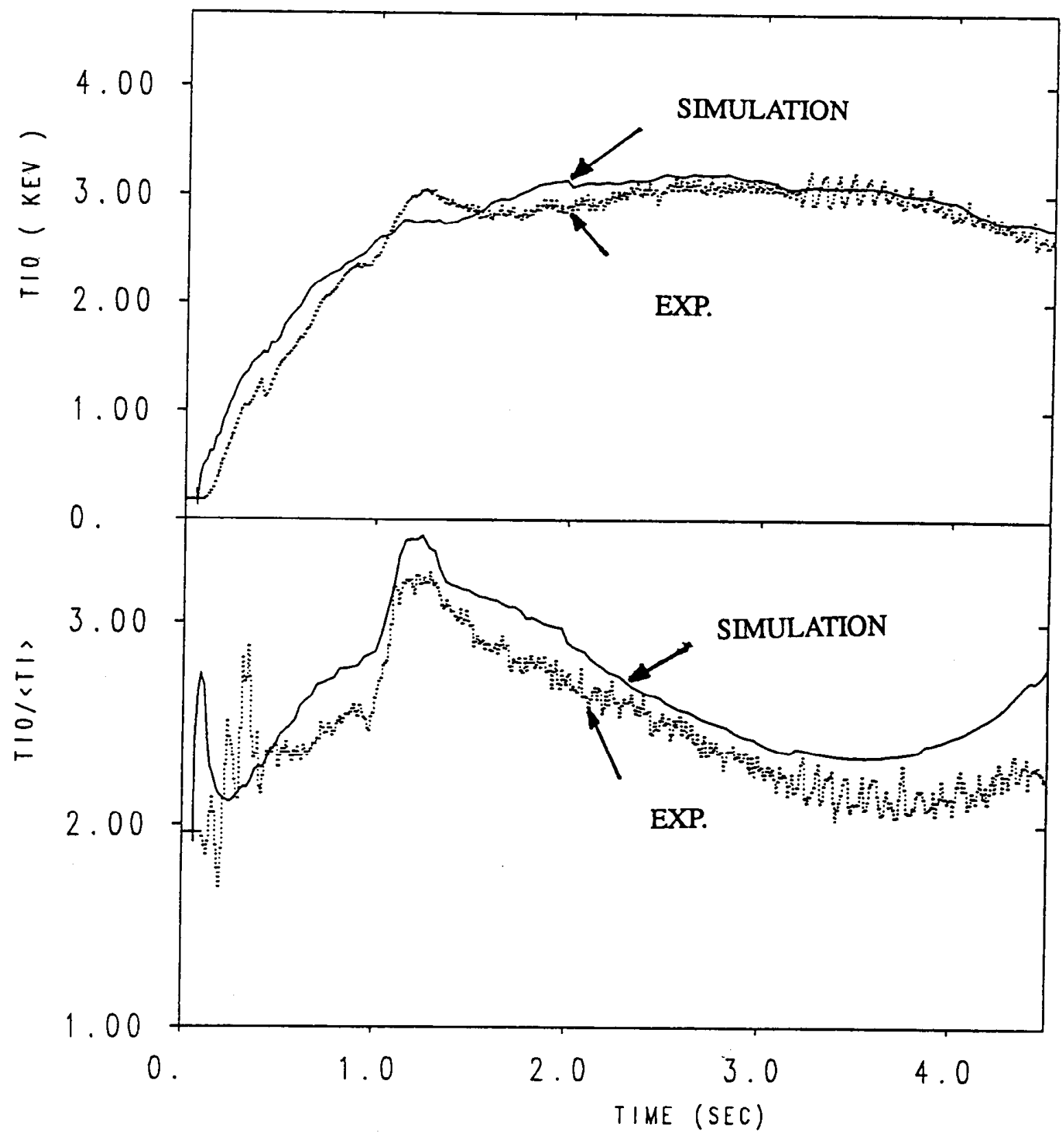

Fig. 5.34. Experiment and simulation values of central ion temperature $T_{i}(0)$, and peak-to-average ion temperature $T_{i}(0) /\left\langle T_{i}\right\rangle$ as a function of time. 


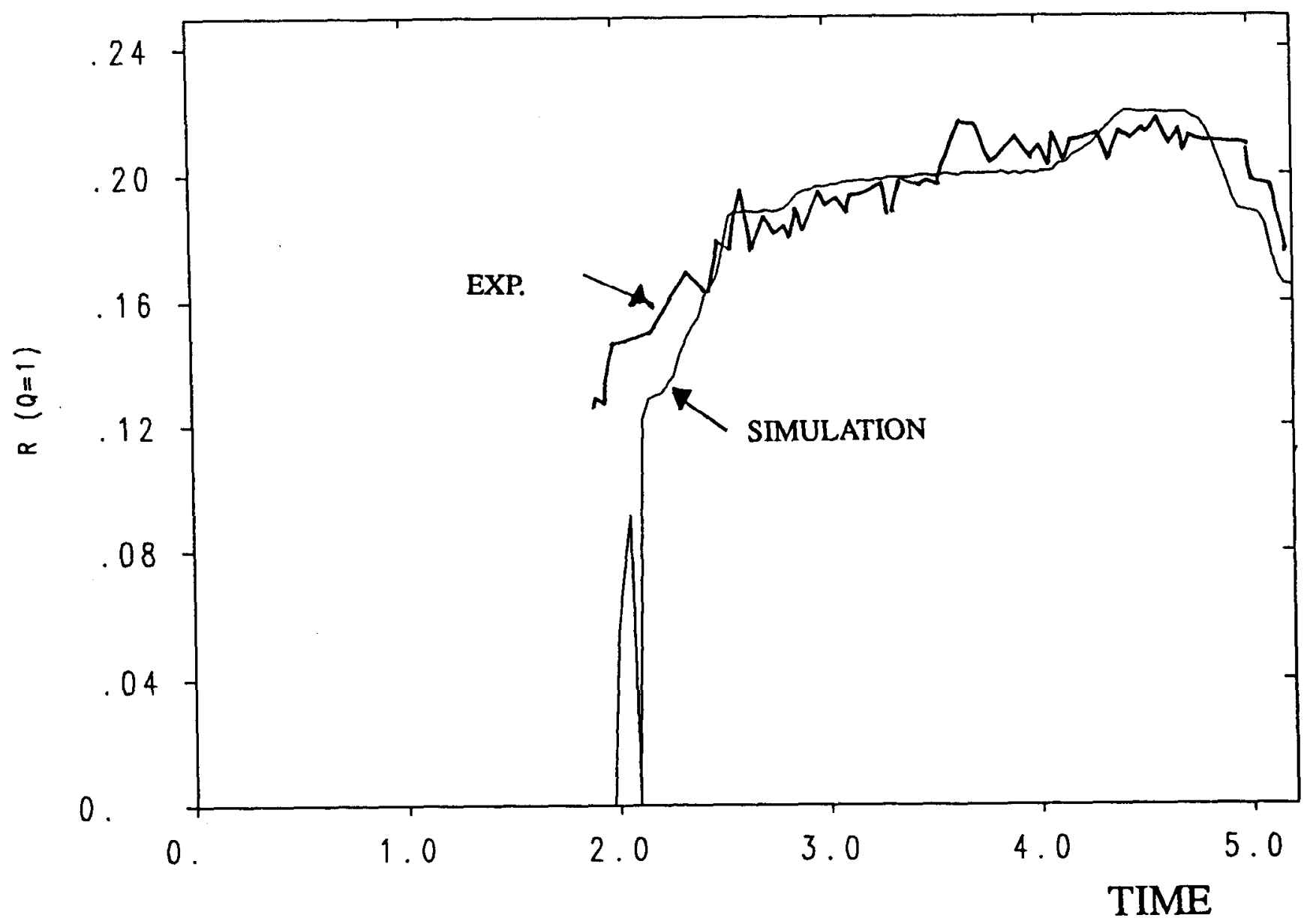

Fig. 5.35. Experiment and simulation values for the $q=1$ radius as a function of time. 

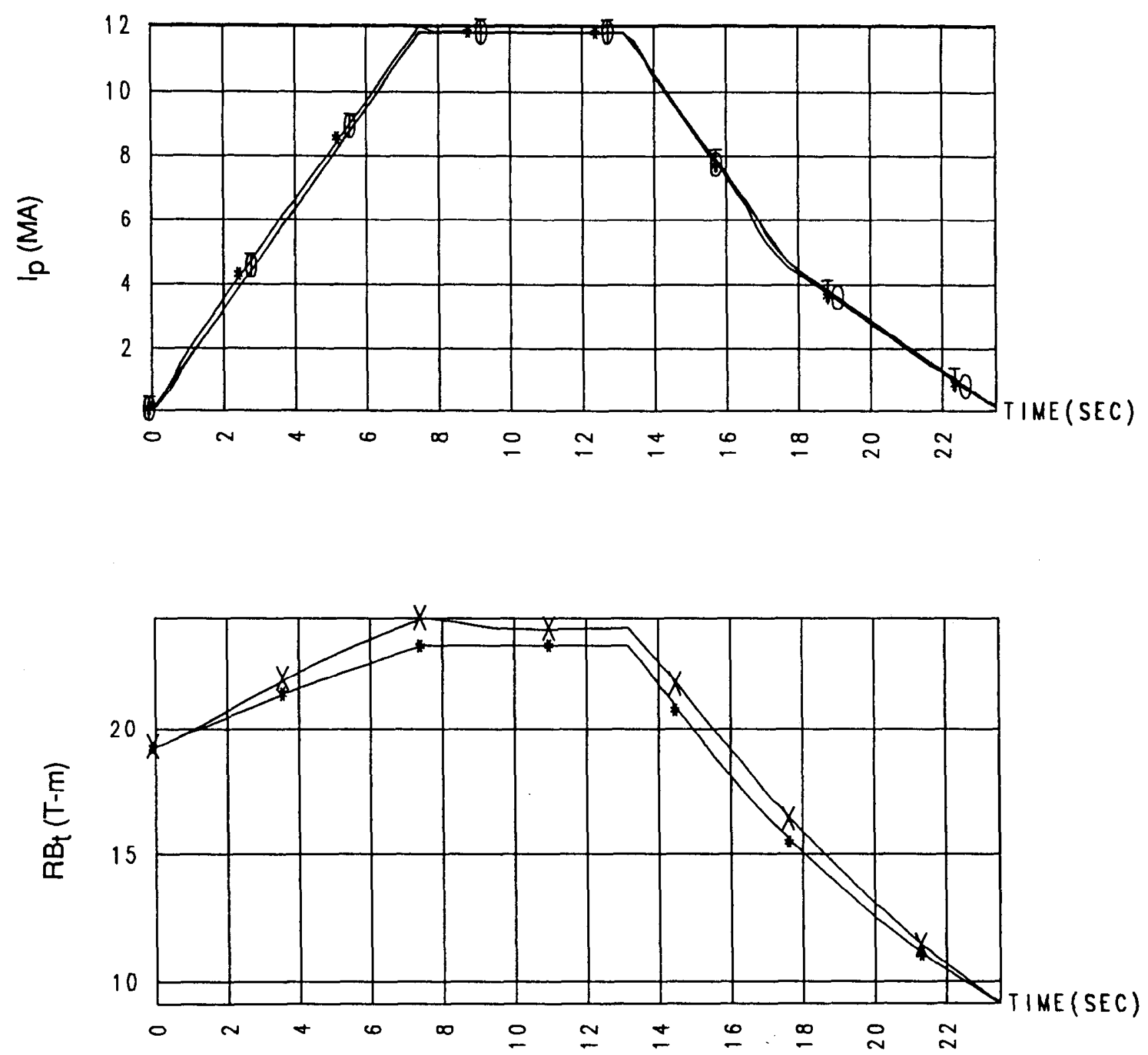

Fig. 5.36. Evolution of plasma current $I_{p}$ and toroidal field (actually $R B_{t}$ ) for the BPX 500-MW DN simulation. Top graph contains three curves indicating preprogrammed (0), and two measures of the actual $\left(T,{ }^{*}\right)$ plasma current. Bottom graph shows $R B_{T}$ due to vacuum field $\left(^{*}\right)$ and at plasma center (X). The difference in the two is due to plasma paramagnetic effect. 


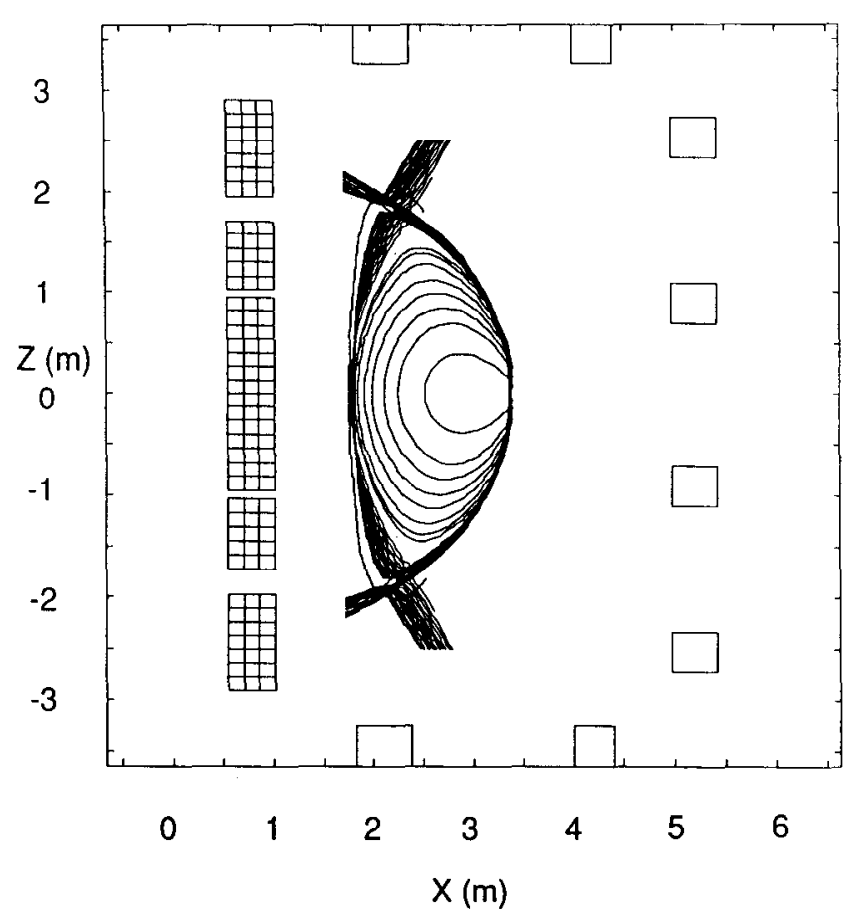

Fig. 5.37. Snapshots of the plasma/vacuum interface at various times during the evolution.

elongation of $\kappa_{95}=2.1$ at the beginning of flattop (BOFT). During the 5.7-s current flattop, the plasma elongation is decreased to 2.0 while the plasma triangularity is increased from $\delta_{95}=0.25$ at BOFT to $\delta_{95}=0.44$ at the end of flattop (EOFT) in order to effect a 20-cm sweep of the outboard separatrix strike-point across the divertor plate surface. Figure 5.38 shows detailed time histories of the shape evolution with line plots of major radius, minor radius, elongation, and triangularity. Figure 5.39 shows the time evolution of strike-point distance along the divertor plate (measured in meters from the inboard midplane) for both the inboard and outboard separatrices. The critical outboard strike-point is seen to sweep a distance of $20 \mathrm{~cm}$. The PF coil currents were programmed to make the outer strike-point velocity greatest when the plasma heat flux is the highest. Also shown in Fig. 5.39 are the separation distances from the plasma X-point to each of the strike-points. Minimum separation distances are $15 \mathrm{~cm}$ for the outboard strike-point and $10 \mathrm{~cm}$ for the inboard strike-point. Upon completion of the divertor sweep, the plasma major radius is immediately decreased to force the plasma to be limited on the inboard edge and thereby decrease the heat load in the divertor region.

Figure 5.40 shows the calculated edge safety factor as a function of time. Several definitions of $q_{\text {edge }}$ are possible. We use a definition of $q_{\text {edge }}$ such that its values vary smoothly when a transition is made from a limiter-limited plasma to a diverted plasma: Let $\psi_{95}$ label the flux surface that encloses $95 \%$ of the flux between the magnetic axis and the nearest X-point to the plasma, and $\psi_{\text {edge }}$ label the plasma/vacuum interface. Let $\psi=0$ at the symmetry axis of the machine and reach a minimum at the plasma magnetic axis. Then $q_{\text {edge }}=q\left(\psi_{\text {edge }}\right)$ if $\psi_{95}>\psi_{\text {edge}}$; otherwise, $q_{\text {edge }}=q\left(\psi_{95}\right)$ if $\psi_{95}<\psi_{\text {edge }}$. With this definition, qedge reduces to the conventional definition for both well-limited and diverted plasmas and provides a smooth interpolation in the transition region. From Fig. 5.40, we see that $q_{\text {edge }}>3.2$ for the entire discharge. In fact, during the critical highbeta period, $q_{95}=3.4$, providing cxtra assurancc against disruptions and confinement degradation at low $q$.

Figure 5.41 shows a plot of the plasma power balance as a function of time. To assist heating of the plasma, $20 \mathrm{MW}$ of auxiliary power is made available to the fiducial simulations. For the $P_{\text {fus }}=500 \mathrm{MW}$ simulations, $10 \mathrm{MW}$ of $P_{a u x}$ is added at $t=4.5 \mathrm{~s}$, and this is increased to $20 \mathrm{MW}$ beginning at $t=5.5 \mathrm{~s}$. The alpha power production is significant at $t=7.0 \mathrm{~s}$ at which time $P_{\alpha}$ first exceeds $P_{a u x}$. When the total heating power, $P_{\text {heat }}=P_{\alpha}+P_{\text {ohmic }}+P_{\text {aux }}$, reaches $100 \mathrm{MW}$, the TSC instantaneously adjusts $P_{a u x}$ in an attempt to maintain $P_{\text {heat }}=100 \mathrm{MW}$. This feedback procedure is seen to be stable. The value $P_{\alpha}=95$ $\mathrm{MW}$ is achieved at $t=10.0 \mathrm{~s}$, after $2.5 \mathrm{~s}$ of plasma current flattop. A slight degradation of $P_{\alpha}$ is seen between the time its maximum is achieved and the end of flattop. The cause of the degradation is not helium ash buildup (this is included in the simulation as a sink term for the density evolution, with an assumed confinement time of $1.0 \mathrm{~s}$ ), but is in fact due to a slow growth of the radius of the $q=1$ surface during the flattop period, as seen in Fig. 5.42. This growing region of poor confinement causes the central temperature to drop, with a consequent decrease in $P_{\alpha}$. The calculated energy confinement time during the steady-state period of the flattop was $\tau_{E}=980 \mathrm{~ms}$, corresponding to an ITER89-P multiplier of 2.11.

It is interesting to note that there may be competing advantages and disadvantages, with respect to BPX performance, of varying the onset time for auxiliary heating. The effect of turning on the heating is to "freeze in" the current profile and, hence, the sawtooth inversion radius. Enhanced transport within the $q=1$ region implies that confinement and profile peakedness are degraded by delaying the onset of heating. On the other hand, TFTR experimental data have shown a tendency for reduced confinement relative to L-mode scal- 
Jardin et al. MHD EQUILIBRIUM AND STABILITY
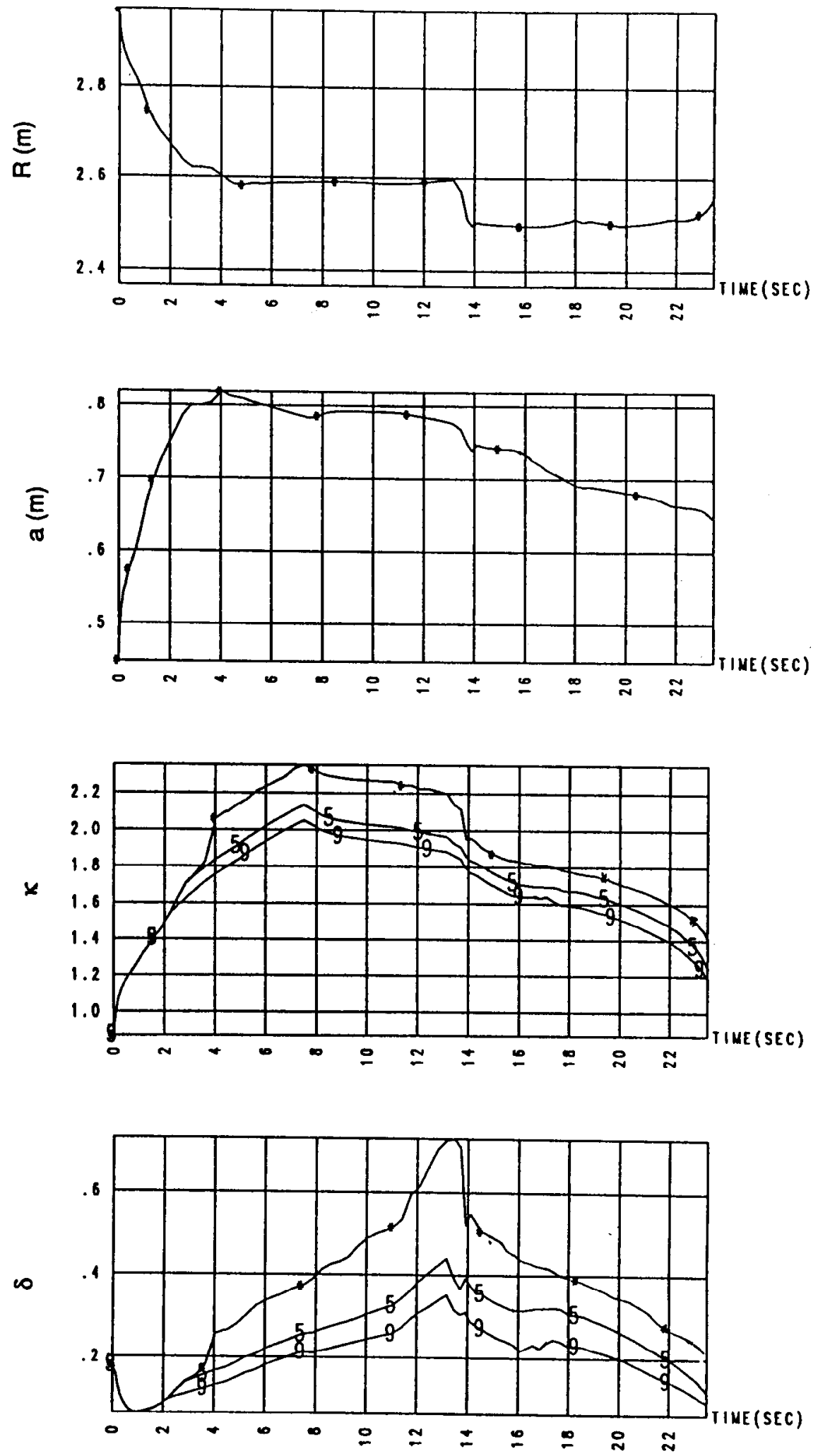

Fig. 5.38. Time histories of major radius $R$, minor radius $a$, elongation $\kappa$, and triangularity $\delta$ for the $500-\mathrm{MW}$ DN simulation. The symbol 5 indicates the $95 \%$ flux surface, 9 indicates the $90 \%$ flux surface, and * indicates the plasma boundary. 

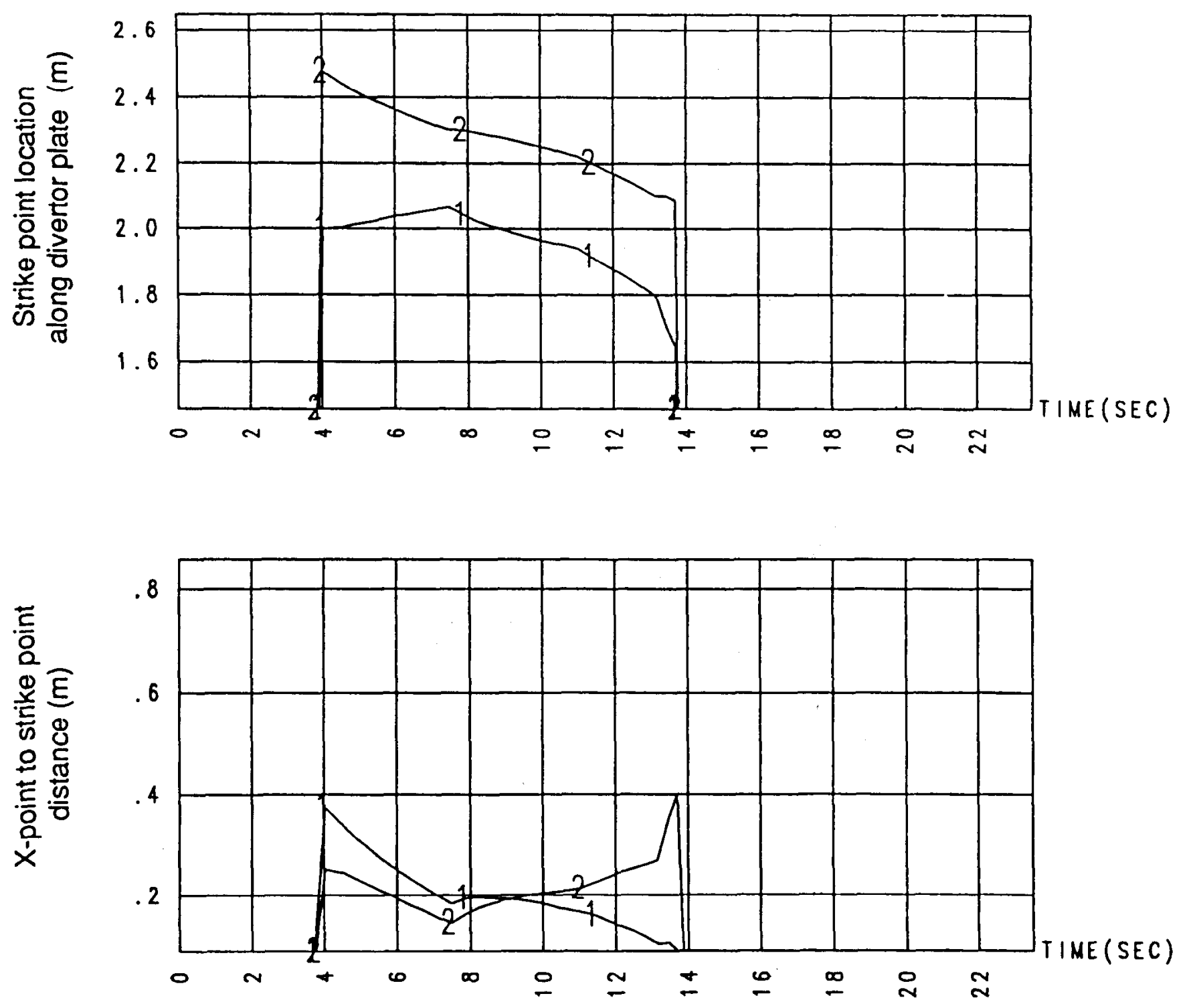

Fig. 5.39. (a) Distance along divertor plate (from inboard midplane) for the inner and outer legs of the plasma separatrix as a function of time and (b) separation distance between the separatrix strike-points on the divertor plate and the plasma null point as a function of time. 


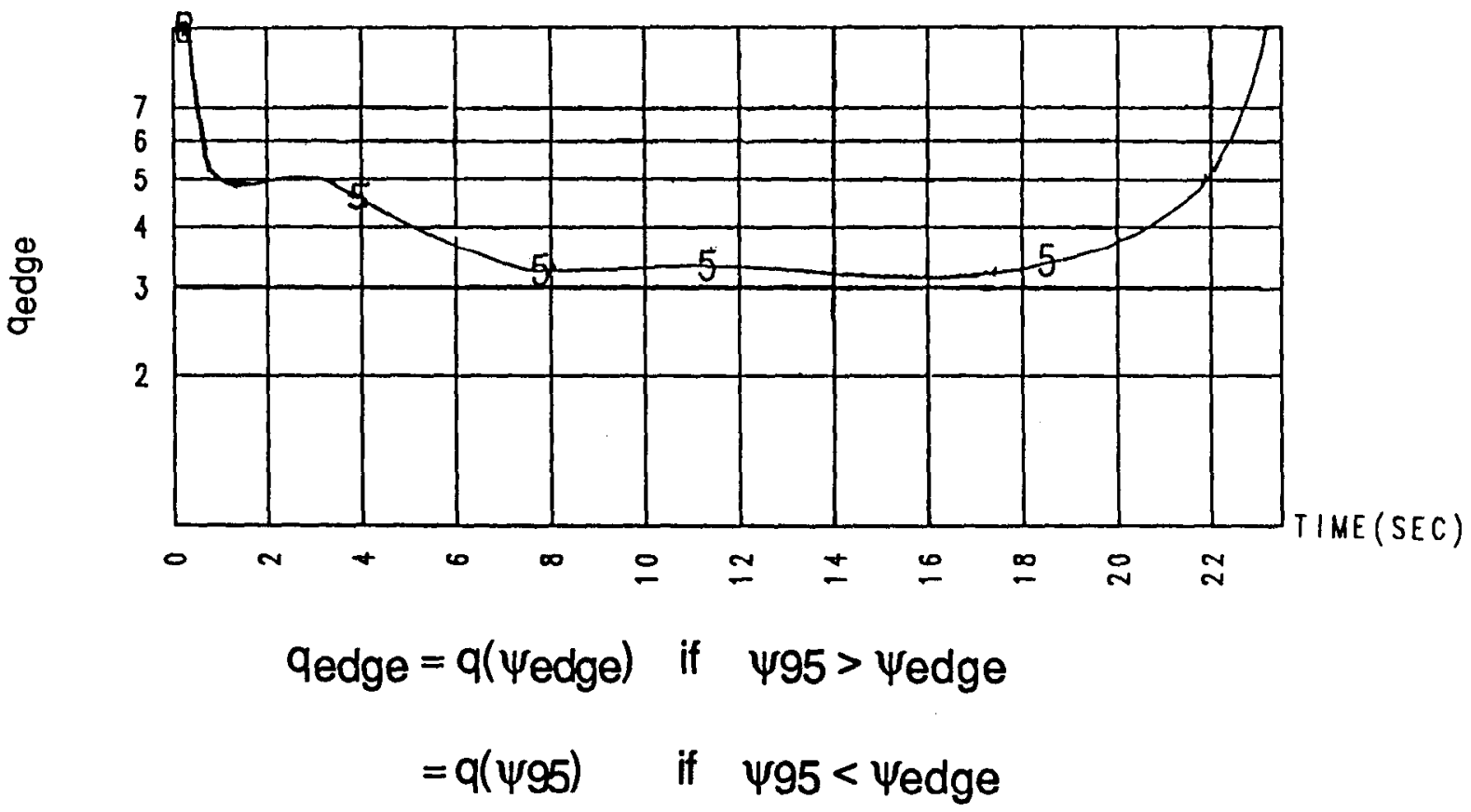

Fig. 5.40. Edge safety factor $q_{95}$ as a function of time.

ing when current profiles are broader. ${ }^{15,16}$ Delaying heating until 2 seconds after beginning of flattop on BPX allows the inversion radius and $\ell_{i}$ to reach their equilibrium values. The BPX device thus has the ability to operate in either mode - investigating both the situation where the sawtooth inversion radius is frozen at a small value and the situation with a fully penetrated current profile.

The plasma density profile assumed for the simulation is shown in Fig. 5.43. Here, the electron, ion, and helium ash densities are shown as functions of poloidal flux at EOFT. The electron and ion density profile shapes are assumed fixed throughout the simulation while the central densities are preprogrammed functions of time. (The helium ash profile is calculated within the TSC code). During the current rampup phase, the central electron density was increased linearly with time to the flattop value $n_{e}(0)=3.6 \times 10^{20} \mathrm{~m}^{-3}$ and held constant during the flattop. Figure 5.44 shows the ratio of volume-average density to the Murakami and Greenwald limits. We see that $\langle n\rangle / n_{M u r} \$ 0.9$ and $\langle n\rangle / n_{\text {Greenwald }} \lesssim 0.5$. To characterize the profile shapes, it is convenient to consider the density profile peaking factor defined as $\alpha_{n}=n_{e}(0) /\left\langle n_{e}\right\rangle-1$. The parameter $\alpha_{n}$ is calculated from Fig. 5.43 to have the value 0.65 during the flattop phase.

The temperature profiles can be characterized in the same way. Figure 5.45 shows a plot of the calculated central electron and ion temperature as a function of time, as well as the peak-to-volume average ratio. The calculated temperature profile peaking factor during the flattop is seen to be $\alpha_{T} \approx$ 2.4 for the electrons and $\alpha_{T} \approx 2.2$ for the ions.

It has been observed on JET that, for a given plasma equilibrium, there is a value of plasma internal inductance $\ell_{i}$ that cannot be exceeded without developing a major disruption. ${ }^{17}$ This limiting condition is conveniently expressed by plotting $\ell_{i} / 2$ as a function of $q_{95}$, since each of these quantities can be related to the MHD stability of tearing and kink modes. Figure 5.46 shows a plot of the evolution of the BPX equilibrium in this space. The shaded region represents the region of instability predicted by the cylindrical model calculations of Cheng et al. ${ }^{18}$ During the current rampup, the BPX equilibrium trajectory is seen to enter the kink-unstable region associated with the $q=4$ surface. However, more detailed MHD stability analysis of the equilibria in this region have shown them to be stable (see Sec. V.I). We note that the BPX equilibrium trajectory remains in the stable region during the entire current rampdown. Furthermore, there is no significant development of skin currents during the rampdown.

A summary of simulation results where both the plasma current rampdown rate and the toroidal field rampdown rate are varied is shown in $\mathrm{Ta}$ ble 5.20. For a satisfactory rampdown, we require (a) the plasma system trajectory in the space of $\ell_{i}$ versus $q_{95}$ must remain below the unstable resistive instability limit during the entire rampdown and 
Jardin et al. MHD EQUILIBRIUM AND STABILITY

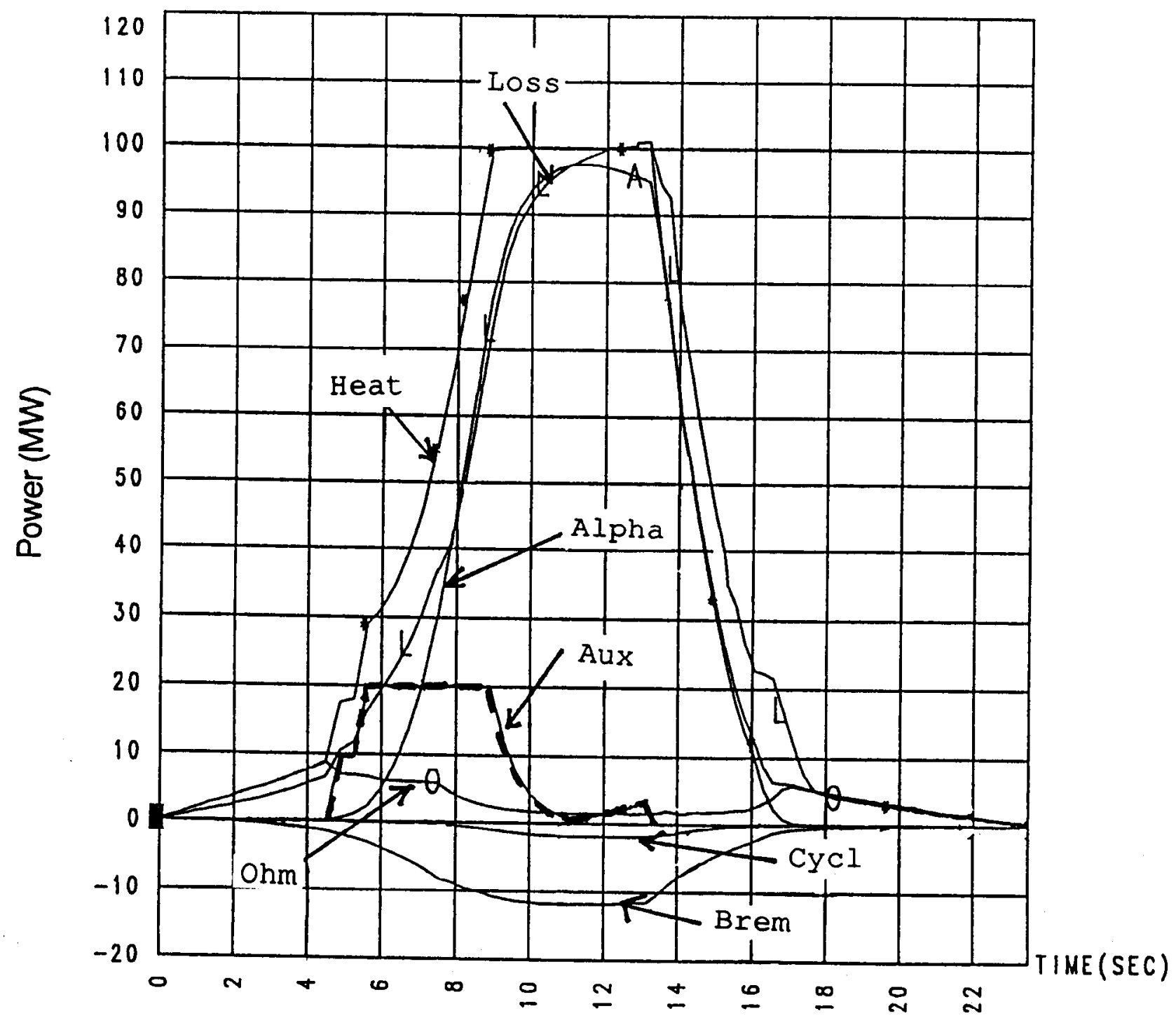

Fig. 5.41. Plasma power balance as a function of time for $P_{f u s}=500 \mathrm{MW}$ DN fiducial simulation.

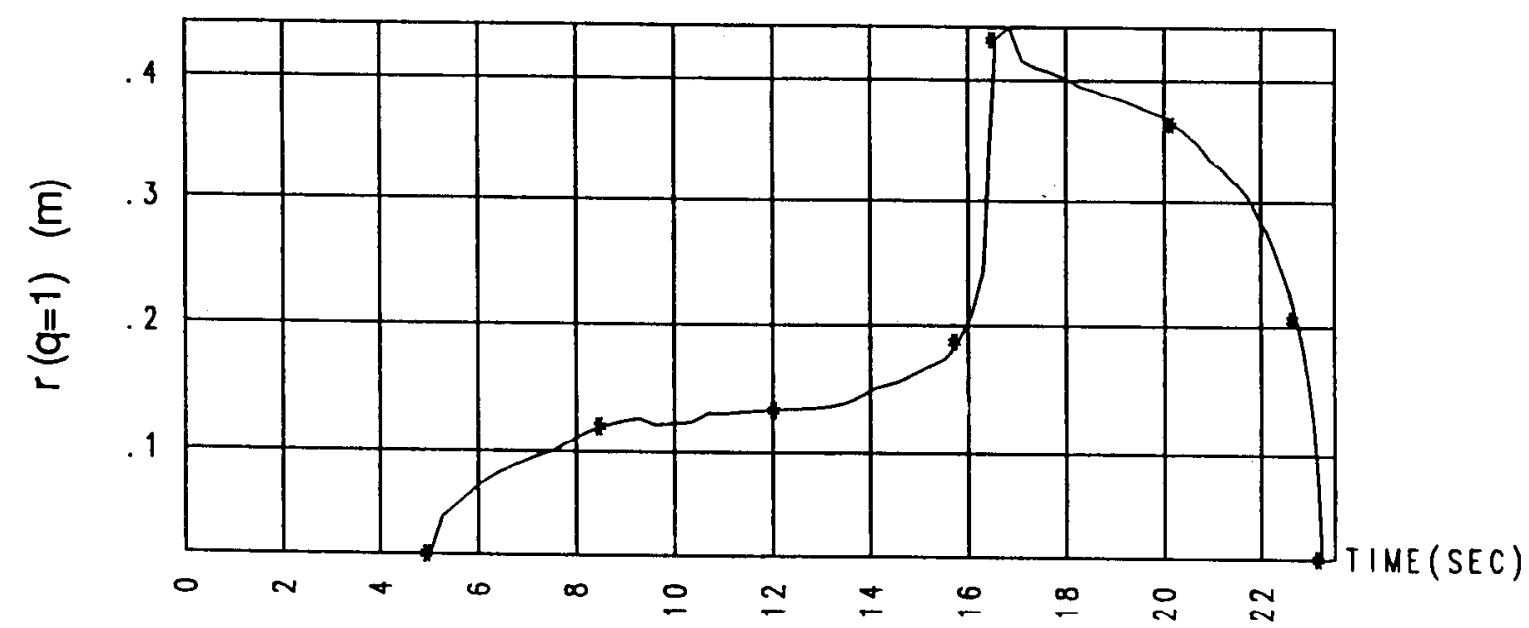

Fig. 5.42. Evolution of the radius of $q=1$ surface. 


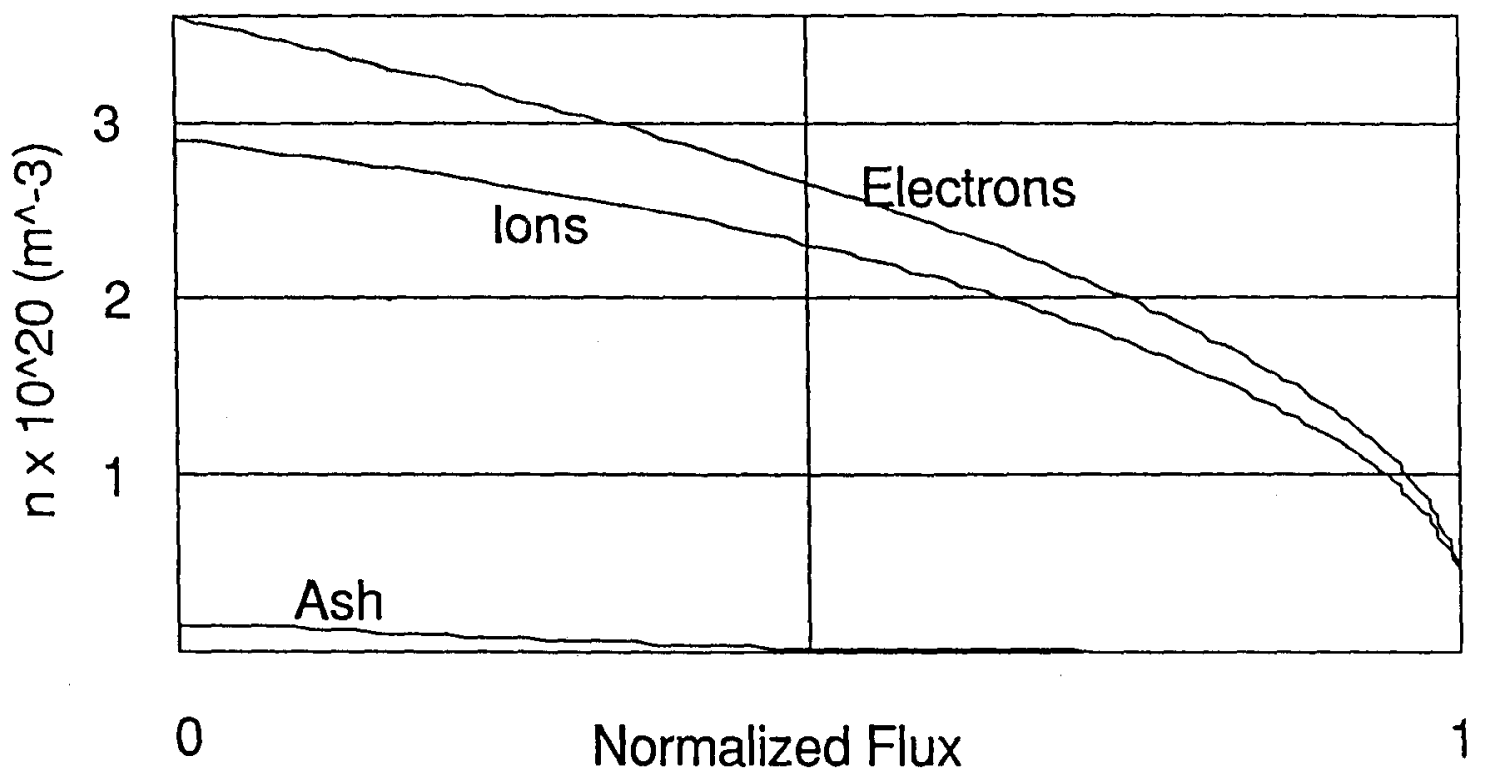

Fig. 5.43. Electron, ion, and helium ash density profiles as a function of poloidal flux at EOFT.

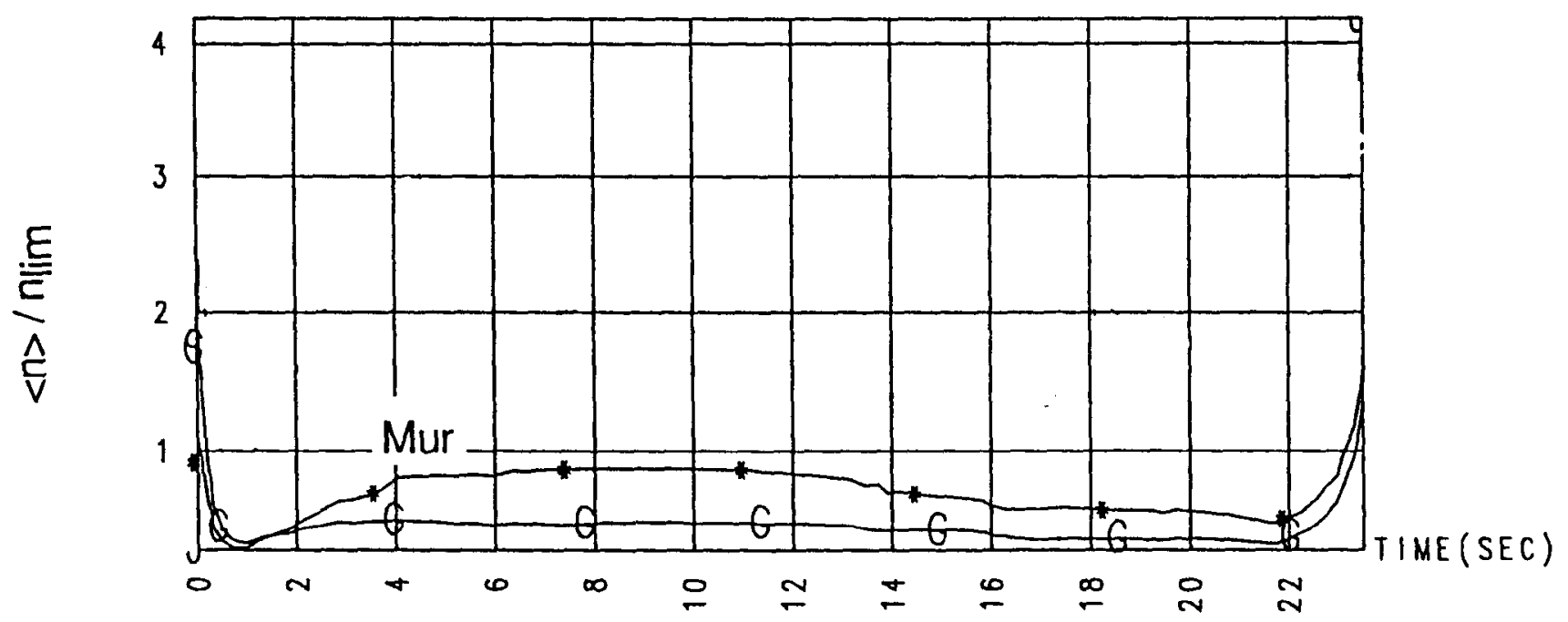

Fig. 5.44. Ratios of volume-average density to the Murakami and Greenwald limits as a function of time. 

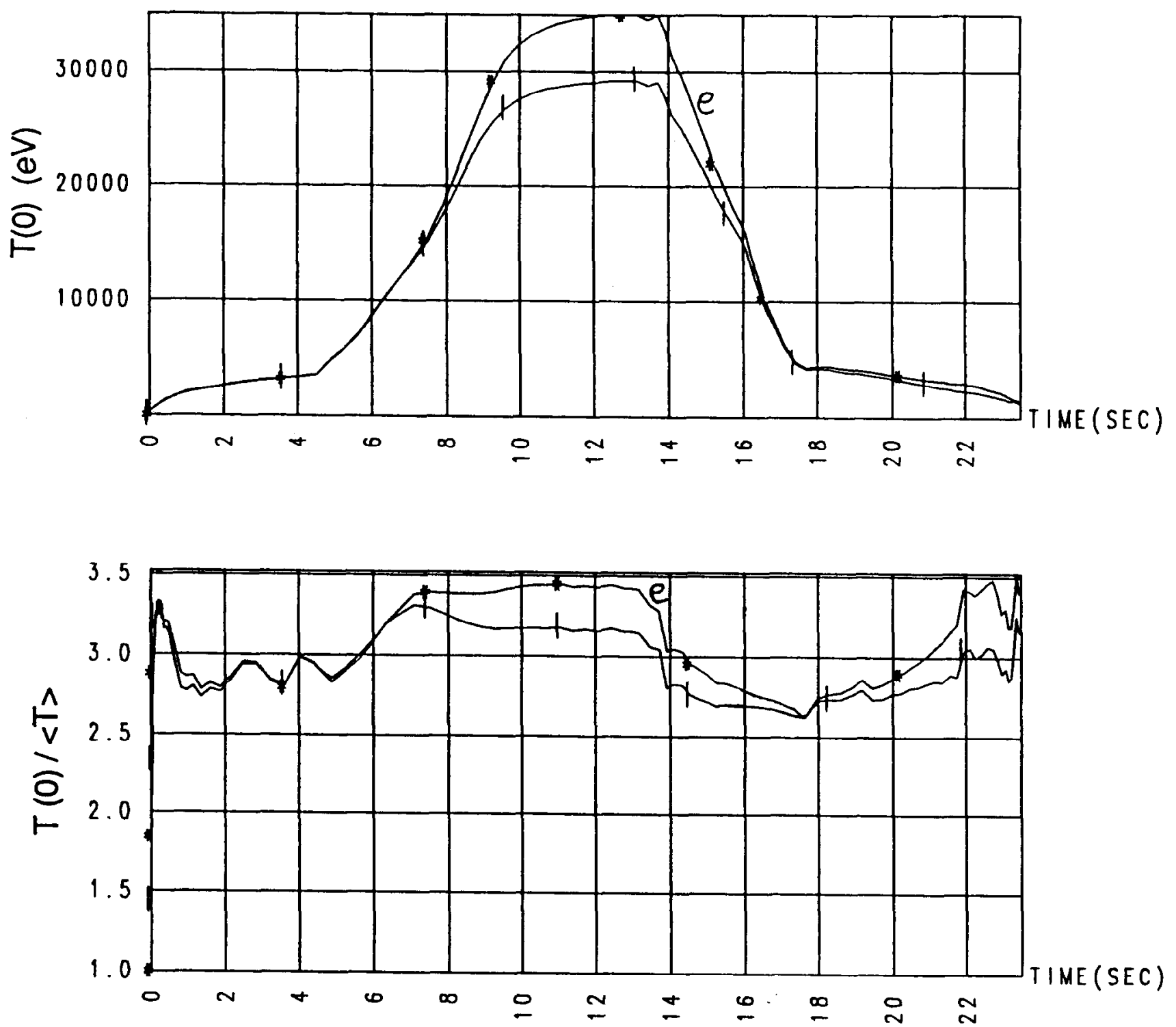

Fig. 5.45. Evolution of central electron and ion temperature $T_{e, i}(0)$ and peak-to-average temperatures $T_{e, i}(0) /\left\langle T_{e, i}\right\rangle$ for the $P_{f u s}=500 \mathrm{MW}$ DN simulation. 


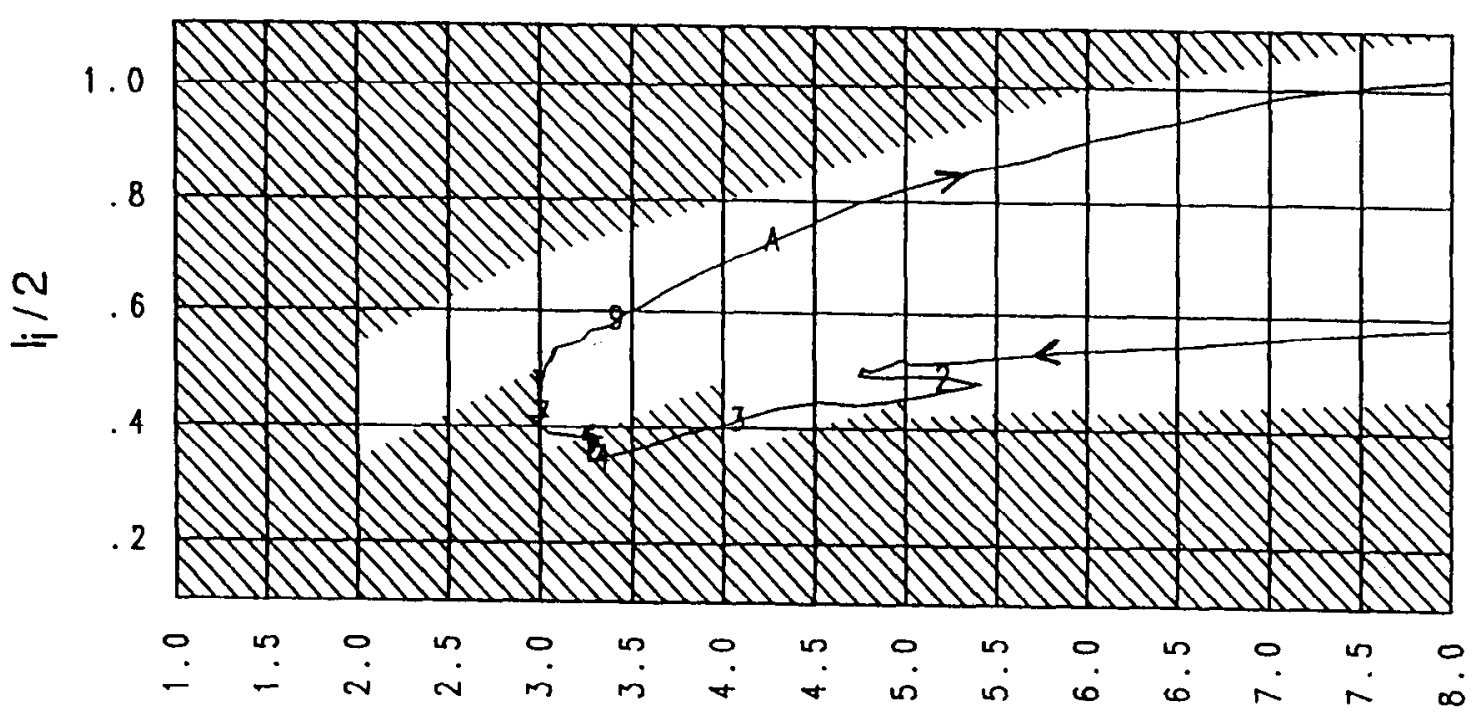

995

Fig. 5.46. Evolution of plasma in space of internal inductance $\ell_{i} / 2$ versus edge safety factor $q_{95}$. Unshaded region is "stable" according to model calculations. ${ }^{18}$ More detailed stability analysis of the equilibria with $q_{95}$ in the range from 3.0 to 4.0 show these equilibria to be stable.

Table 5.20. TSC Simulation Results for Different Rampdown Scenarios

\begin{tabular}{|c|c|c|r|}
\hline Run & $\begin{array}{c}I_{p} \text { rampdown } \\
\text { time }\end{array}$ & $\begin{array}{c}\text { TF rampdown } \\
\text { time const }\end{array}$ & Comment $^{a}$ \\
\hline 1 & 7.5 & 23.0 & NG (3.1) \\
2 & 10.5 & 23.0 & NG (2.2) \\
3 & 13.5 & 23.0 & S (0.0) \\
4 & 7.5 & 11.0 & NG (2.3) \\
5 & 10.5 & 11.0 & M (0.0) \\
6 & 13.5 & 11.0 & S (0.0) \\
\hline
\end{tabular}

${ }^{a} \mathrm{NG}=$ No Good, $\mathrm{M}=$ Marginal, and $\mathrm{S}=$ Satisfactory. The value in parentheses is the amount of plasma current left when the $\ell_{i}-q$ trajectory reaches the unstable boundary.

(b) there should be no substantial development of skin current. For fixed TF ramp rate, we would expect a benefit from slowing $d I_{p} / d t$ since $q$ is increased, and skin currents have longer to diffuse. Thus, runs 1, 2, and 3 show a progressive improve- ment, as do runs 4,5, and 6 . Naively, one might expect that if the $I_{p}$ rampdown rate is fixed and the TF rate is increased, then the situation would be worse since $q$ is decreased. However, the table shows that the situation is actually improved because we get a significant decrease in $\ell_{i}$ (and the amount of skin current is reduced because flux has been peeled off the plasma edge).

The evolution of plasma internal inductance $\ell_{i}$, poloidal beta $\beta_{\text {pol }}$, and volume-average $\beta$ are displayed in Fig. 5.47.

Finally, Fig. 5.48 displays the PF coil currents that effected the simulation. A total of $68.6 \mathrm{~V} \cdot \mathrm{s}$ were required by the PF system. These are provided predominantly by coils PF1, 2 , and 3 swinging in the direction of increasing negative current. During the plasma current flattop, however, PF2 is seen to reverse direction in order to aid the divertor sweep. Since this serves to subtract volt-seconds, $\mathrm{PF} 1$ must continue to increase to compensate.

\section{V.G.2. $P_{f u s}=100 \mathrm{MW}$ DN Divertor}

The 100-MW DN case represents the minimum level of performance required in BPX. However, with respect to volt-second consumption, it is the most demanding, because of the reduced plasma 

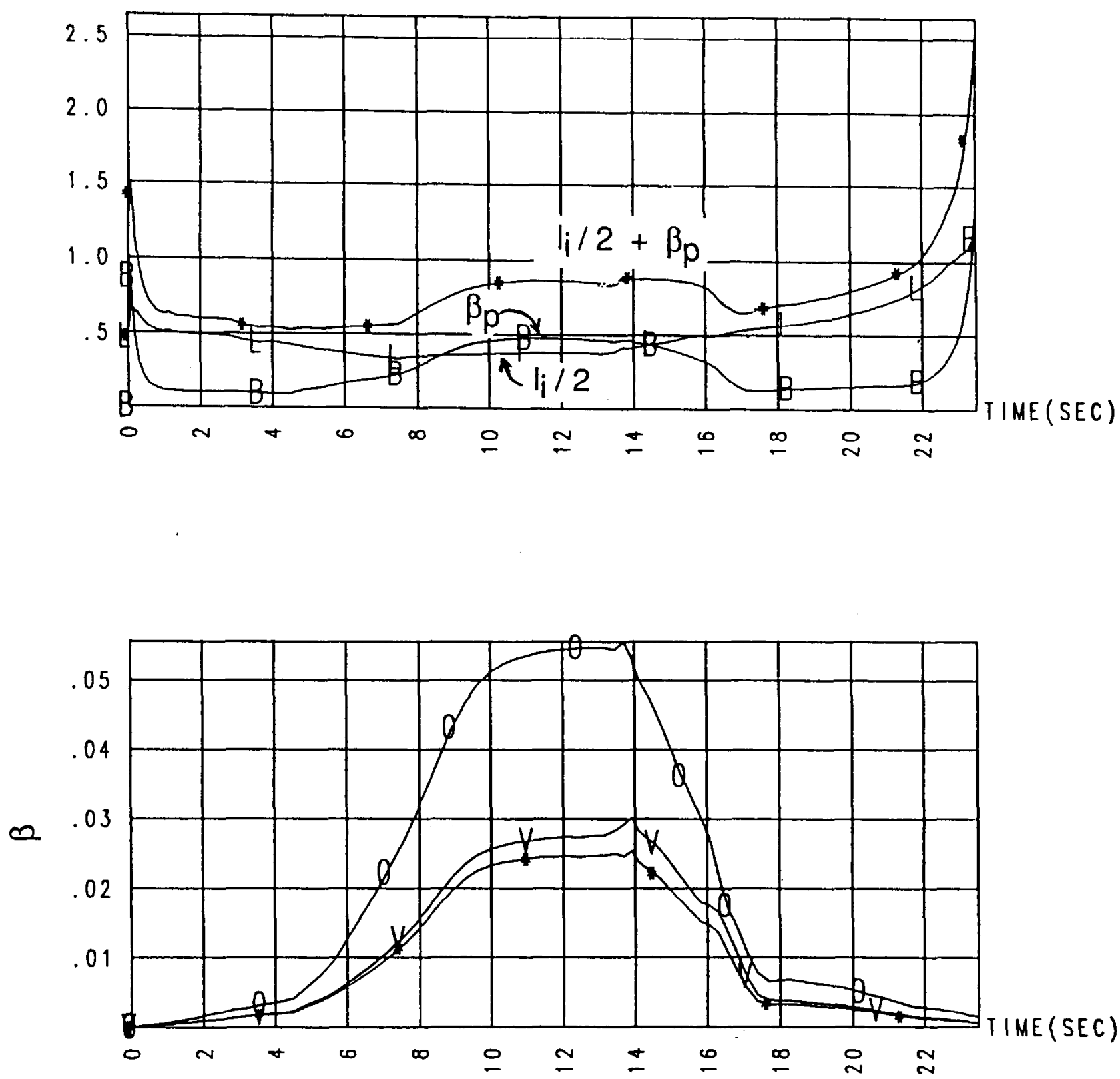

Fig. 5.47. (Top) Evolution of internal inductance $\ell_{i} / 2$, poloidal beta $\beta_{p o l}$, and the sum $\Lambda=\ell_{i} / 2+\beta_{\text {pol }}$. (Bottom) Evolution of one-half central beta (o), beta based on vacuum field (v), and beta based on actual toroidal field $\left(^{*}\right)$. 
Jardin et al. MHD EQUILIBRIUM AND STABILITY

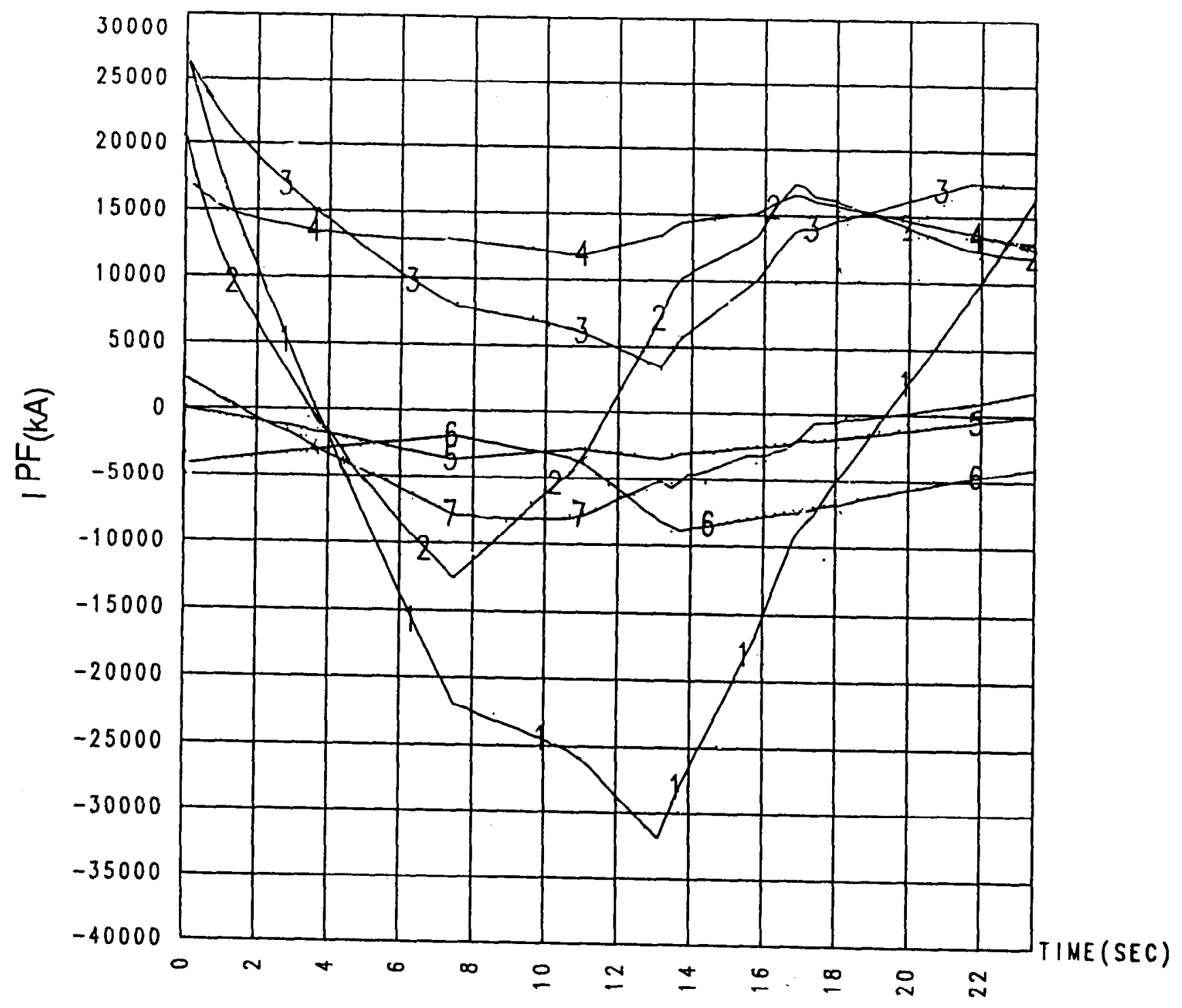

Fig. 5.48. Time histories of PF coil currents (upper + lower half plane values) for the $P_{\text {fus }}=500 \mathrm{MW}$
DN simulation. 


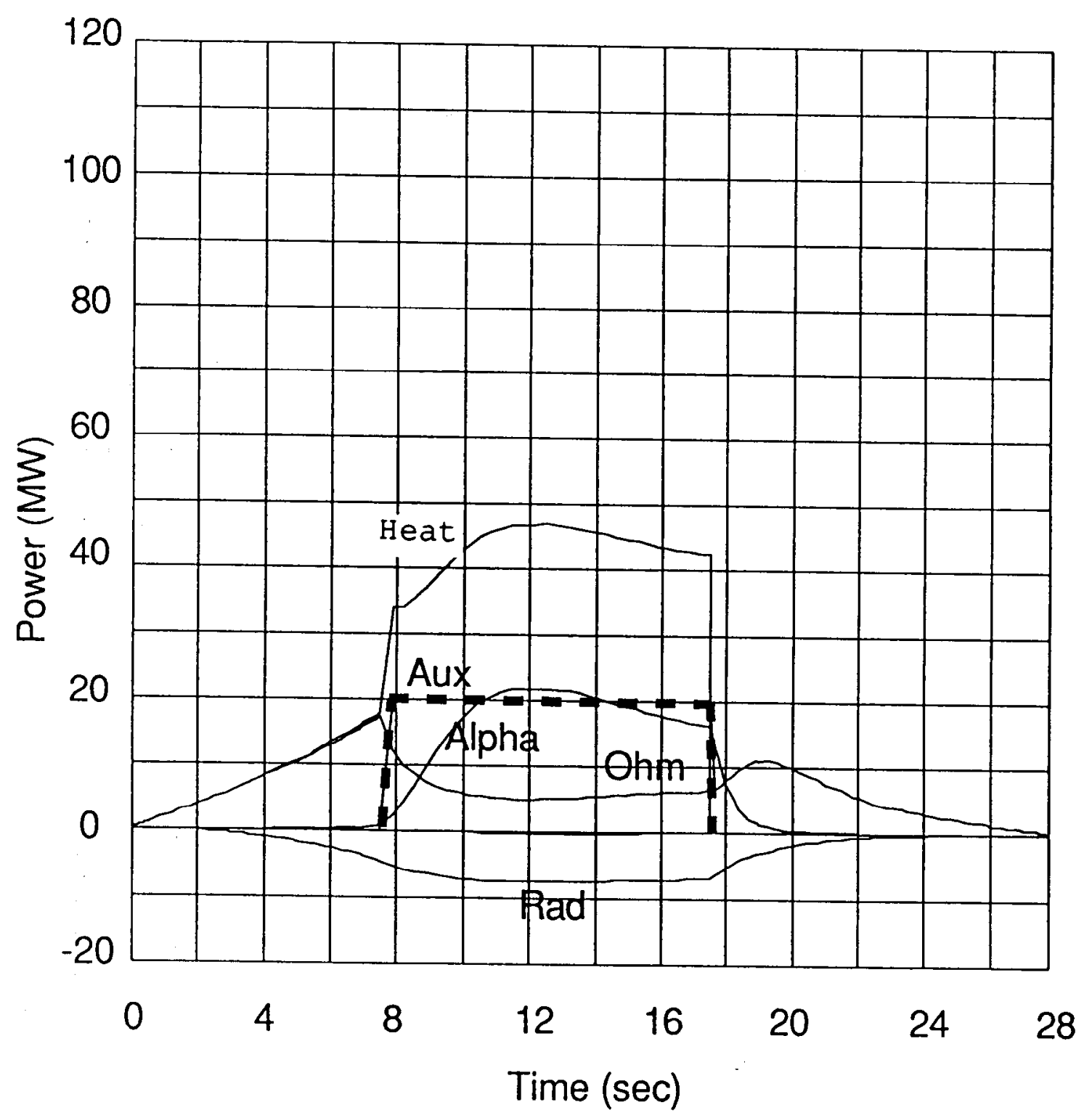

Fig. 5.49. Plasma power balance as a function of time for the $P_{f u s}=100 \mathrm{MW}$ DN fiducial simulation. 
Jardin et al. MHD EQUILIBRIUM AND STABILITY
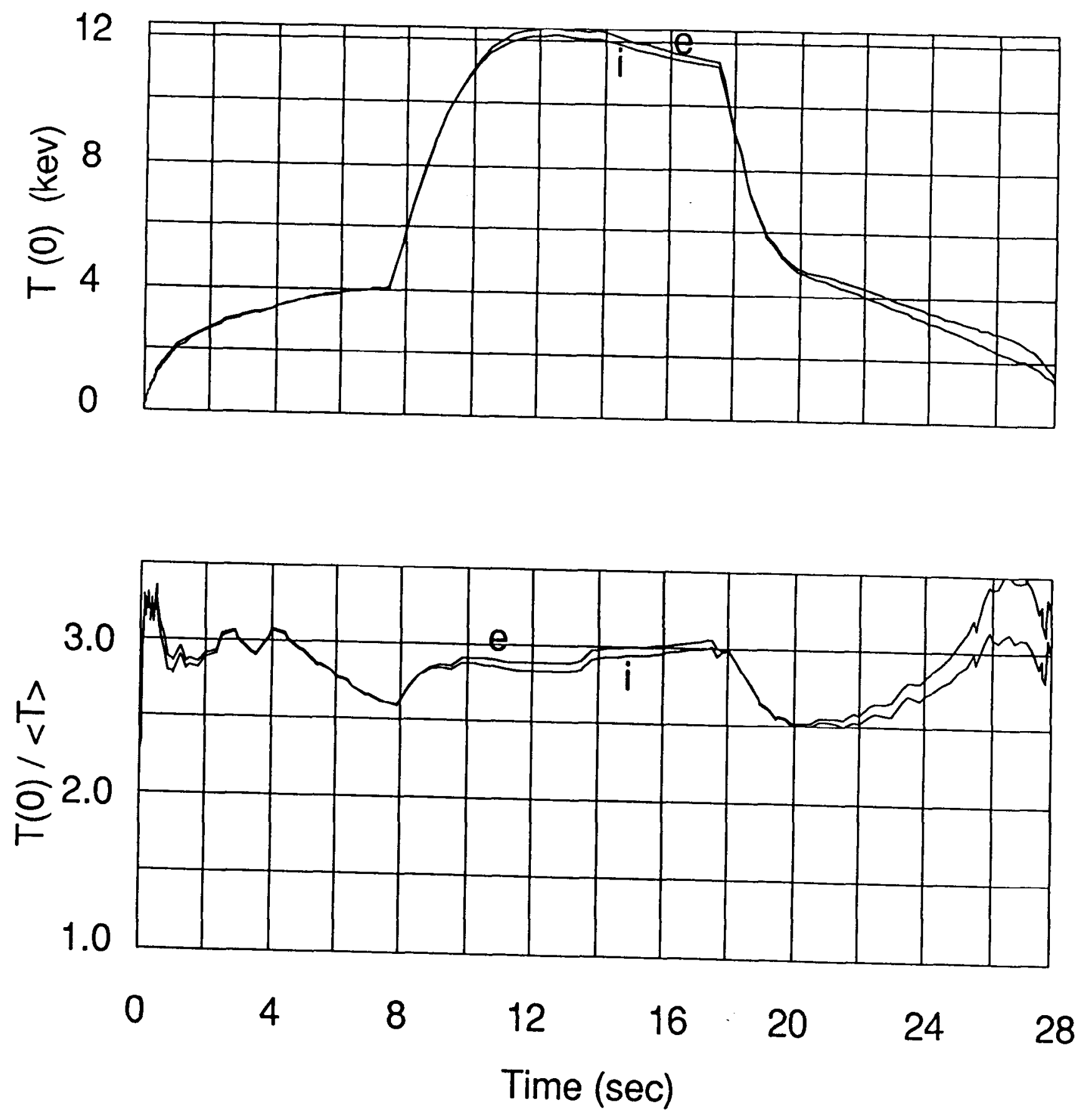

Fig. 5.50. Evolution of central electron and ion temperature $T_{e, i}(0)$ and peak-to-average temperatures
$T_{e, i}(0) /\left\langle T_{e, i}\right\rangle$ for the $P_{f u s}=100 \mathrm{MW} \mathrm{DN}$ simulation. 


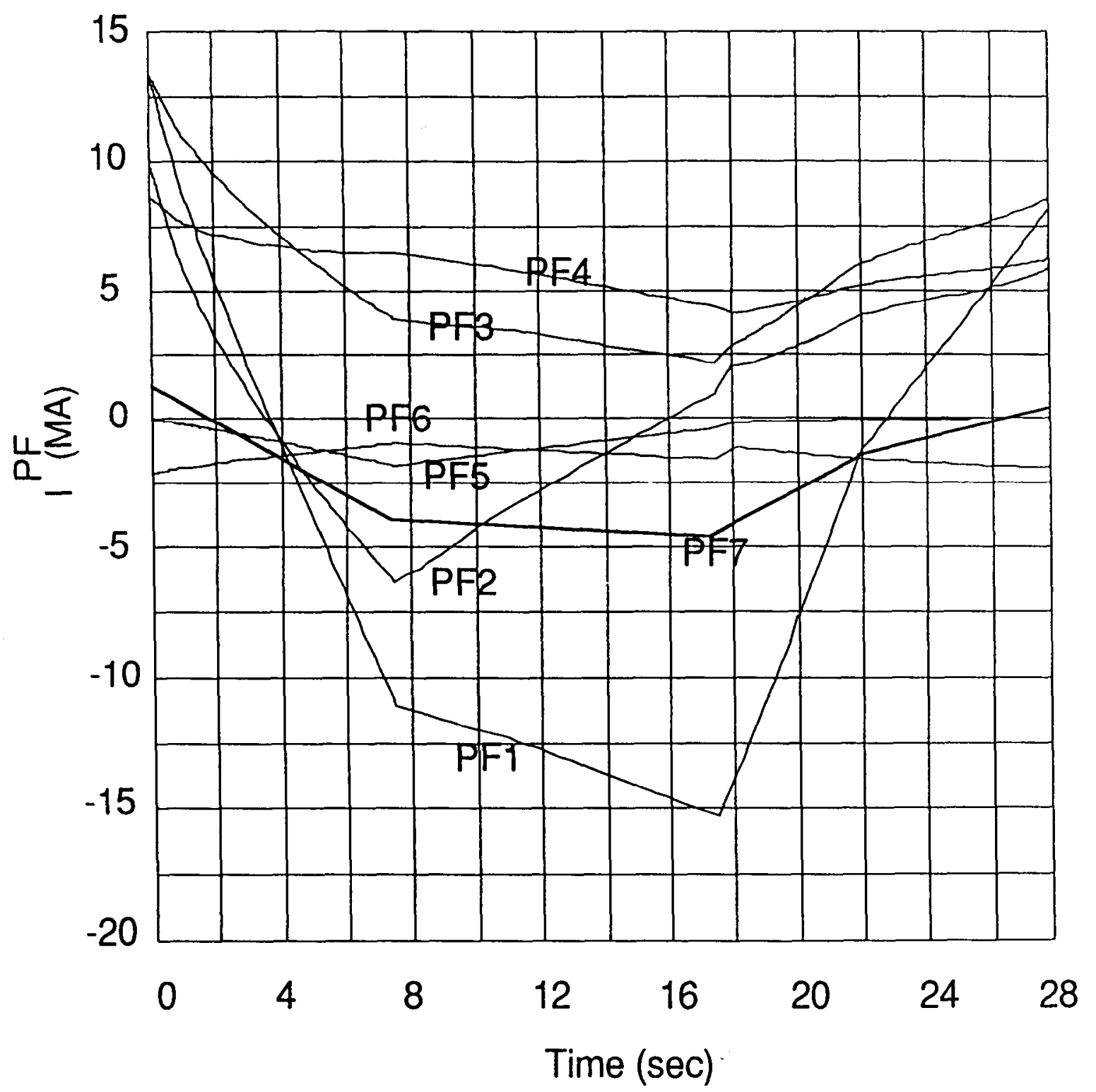

Fig. 5.51. Time histories of PF coil currents (upper + lower half plane values) for the $P_{\text {fus }}=100 \mathrm{MW}$ DN simulation. 
Jardin et al. MHD EQUILIBRIUM AND STABIIITY
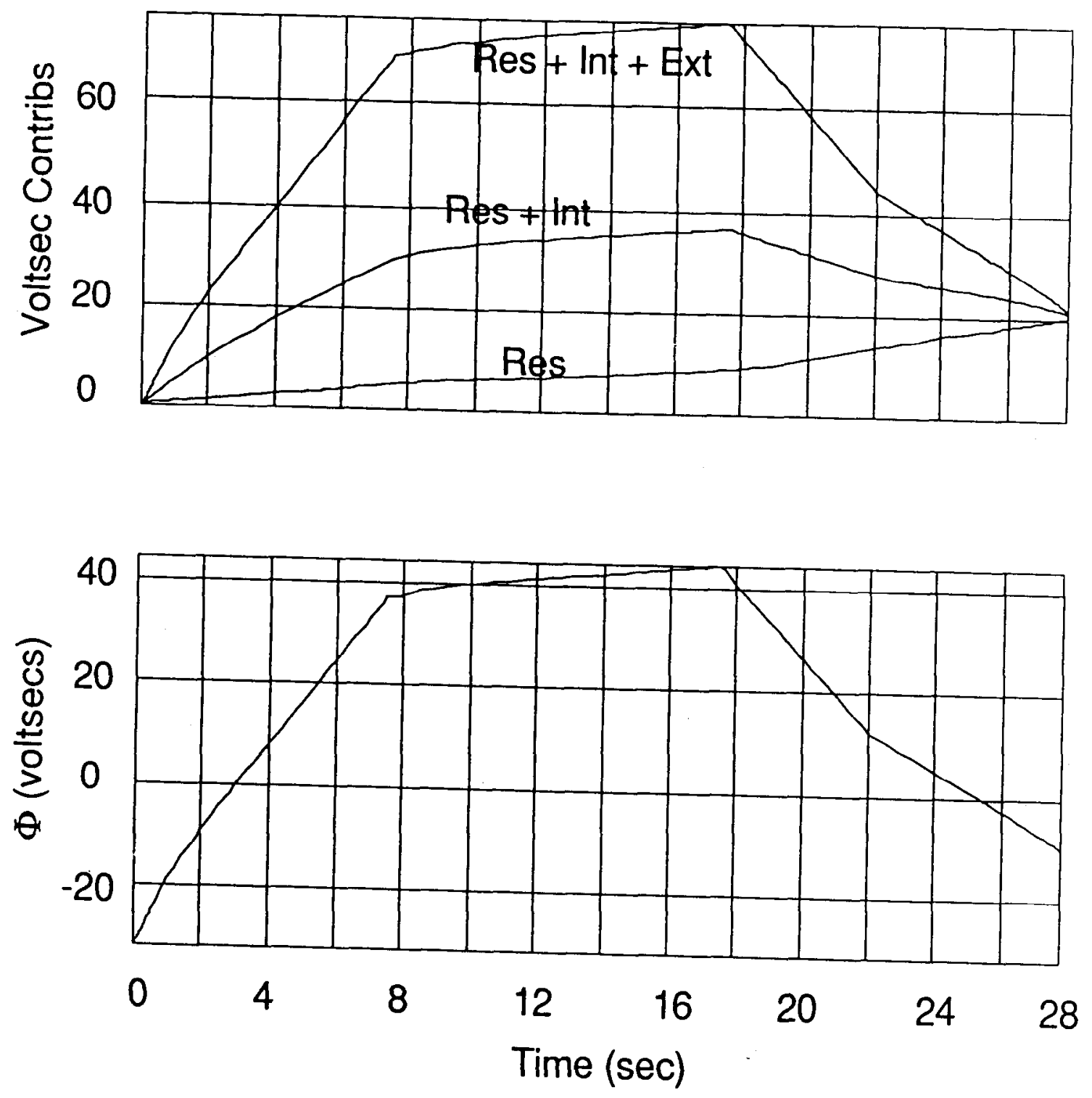

Fig. 5.52. Volt-second accounting for the $P_{f u s}=100 \mathrm{MW}$ DN simulation. 
temperature and the requirement for the full 10-s flattop.

For this simulation, the trajectories for the plasma current, toroidal field, shape, and density were chosen to be the same as for the 500-MW DN simulation except that the plasma current and toroidal field flattop was extended until $t=17.5 \mathrm{~s}$ to obtain a full 10-s flattop. The rampdown therefore extends until $t=28.0 \mathrm{~s}$. The essential purpose of this simulation is to determine volt-second requirements under conservative assumptions. Consistent with the conservative approach, the onset of auxiliary heating was delayed until BOFT.

The target average alpha power production is $P_{\alpha}=20 \mathrm{MW}$, and Fig. 5.49 shows a plot of the time-dependent power balance for this simulation. The degradation of $P_{\alpha}$ during flattop is more pronounced than for the 500-MW simulation, and once again, the reason for the degradation appears to be the growth of the $q=1$ surface. Figure 5.50 shows the evolution of the central electron and ion temperatures, as well as peak-to-average ratios.

Figure 5.51 shows a plot of the coil currents, and Fig. 5.52 shows a plot of the volt-second consumption. A total of $76.2 \mathrm{~V} \cdot \mathrm{s}$ is required by the $\mathrm{PF}$ system to reach EOFT, of which $9.0 \mathrm{~V} \cdot \mathrm{s}$ were due to resistive losses.

\section{V.G.3. $P_{f u s}=500 \mathrm{MW}$ Limiter}

The 500-MW limiter simulation serves a purpose similar to that of the 500-MW DN case, namely, to provide the magnetics information needed to evaluate the power handling capability of the inner wall limiter. The case presented here has the full 10$s$ flattop for $I_{p}$ and $B_{T}$. Preliminary analysis indicates that the limiter capability exceeds its 3-s burn flattop requirement by a significant amount, but analysis to determine whether it can withstand the full-length scenario described here is still in progress at the time of writing.

The parameters for this simulation are identical to the 100-MW DN simulation except that the shape history is chosen to conform to a limiter geometry. Figure 5.53 shows the history of the shape evolution. The triangularity at BOFT is 0.35 and is maintained constant during the flattop. Figure 5.54 shows the coil current history. Because of the demand for high triangularity at BOFT, the currents in coils PF2, 3, and 4 have current values approximately the same as at EOFT for the 500-MW DN case. Further, since the triangularity is held approximately constant during the flattop, these coil currents require only a modest change during the flattop period, which allows a modest change in PF1 also.
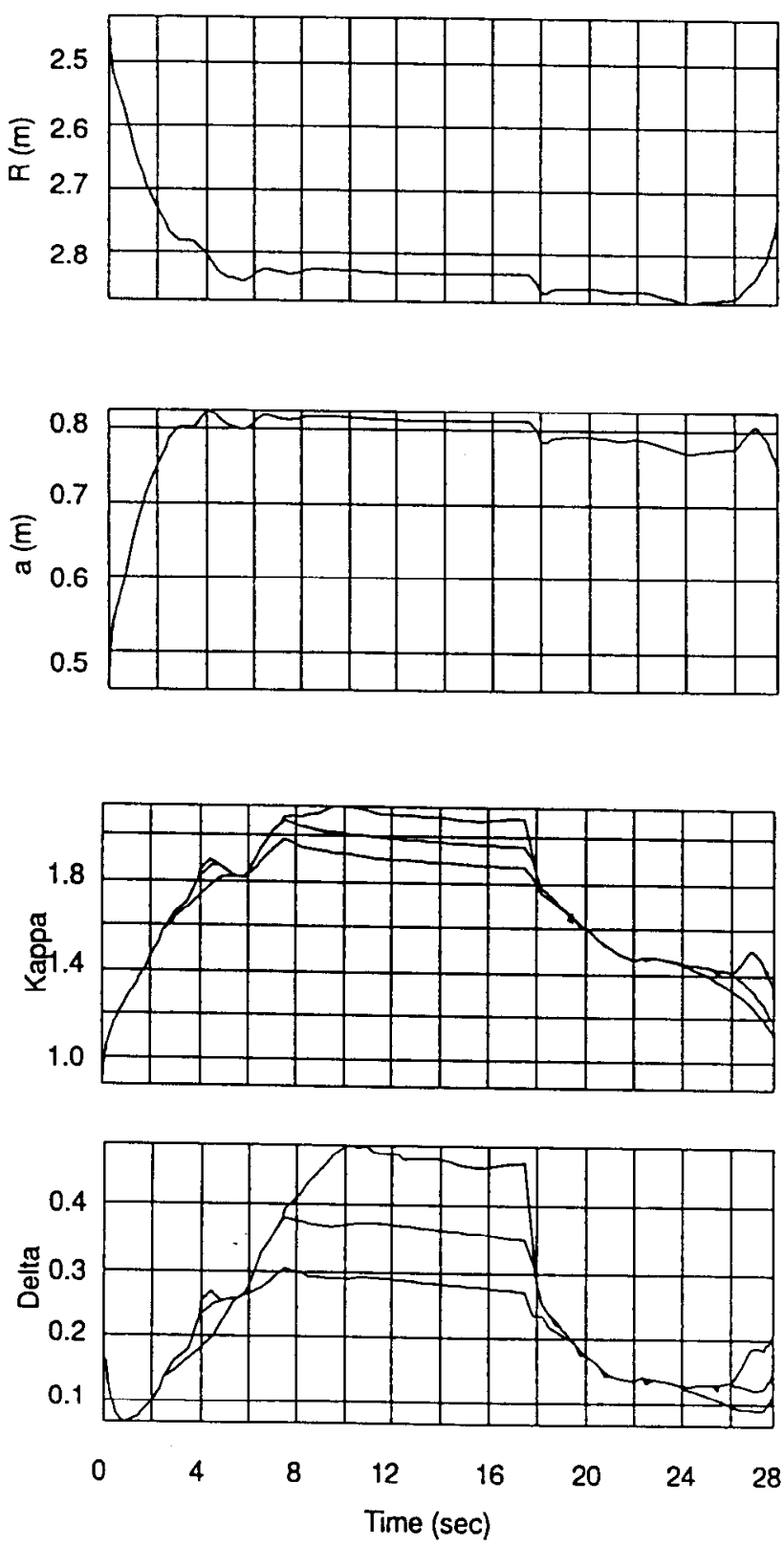

Fig. 5.53. Evolution of major radius $R$, minor radius $a$, elongation $\kappa$, and triangularity $\delta$ for the $P_{\text {fus }}=500 \mathrm{MW}$ limiter simulation. 
Jardin et al. MHD EQUILIBRIUM AND STABILITY

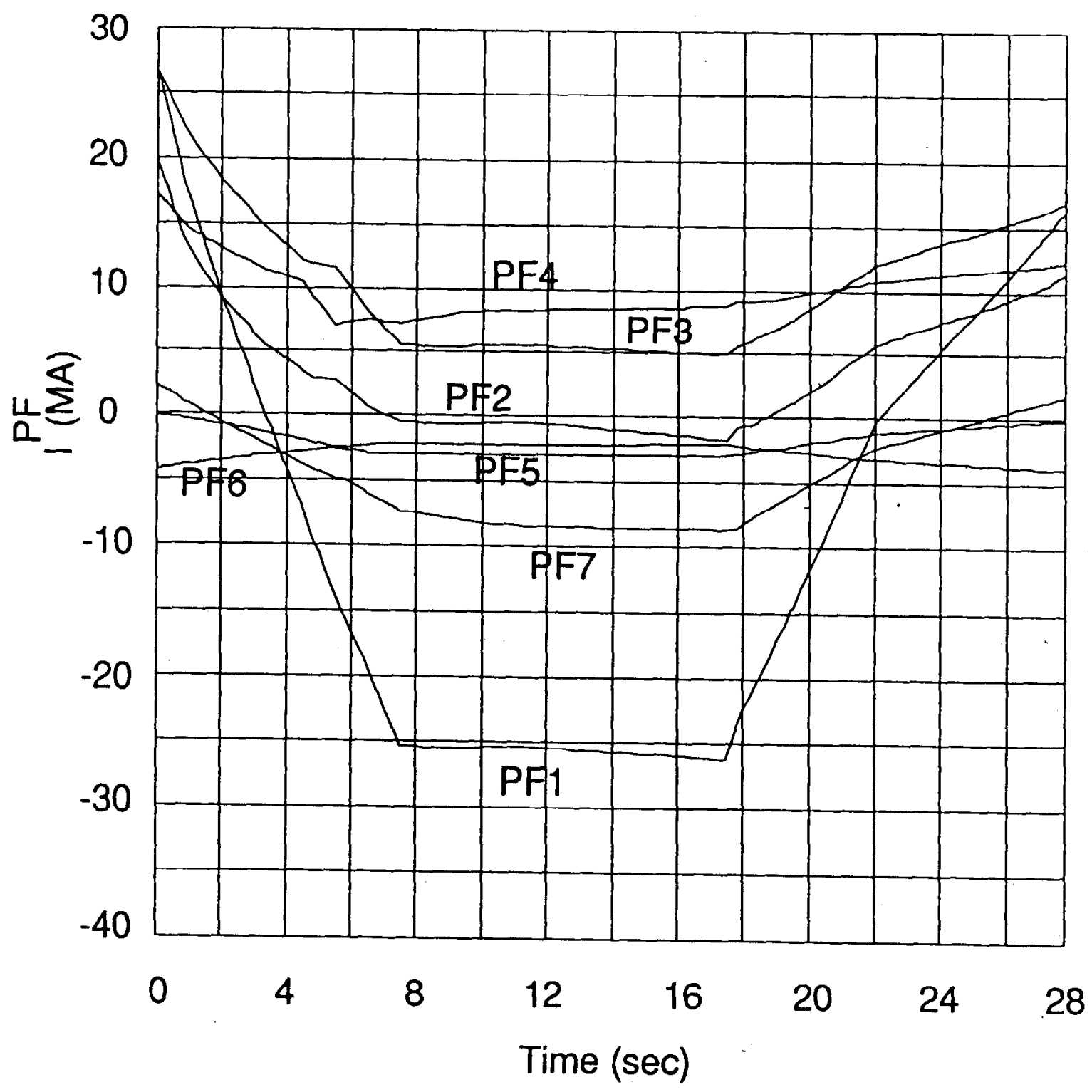
Fig. 5.54. Time histories of PF coil currents (upper + lower half plane values) for the $P_{f u s}=500 \mathrm{MW}$
limiter simulation. 


\section{V.H. PLASMA DISRUPTIONS}

The design of the BPX vacuum vessel is strongly driven by the need to withstand disruption-induced forces produced by sudden plasma motion and current decay. Accurate predictions of the time behavior of disruptive effects are therefore needed. To provide this capability, we characterize the behavior of the disrupting plasma by using a simulation code with a semi-empirical disruption model that is capable of reproducing key features observed in disrupting plasmas in existing tokamaks.

One of the most important parameters describing an experimental plasma disruption is the current decay rate, $d I_{p} / d t$. As shown in Fig. 5.55, taken from a TFTR disruption, this decay rate is not normally constant in time during a disruption, but can be characterized by an average and a peak rate of current decay. By comparing these current decay rates for many disruptions on several machines, a tendency is observed for both the peak and the average decay rates to increase with increasing plasma current. This is illustrated in Fig. 5.56, which graphs the peak rates during the disruption versus critical plasma current for TFTR, and in Fig. 5.57, which shows the average current decay rate versus initial plasma current for JET, JT-60, and TFTR. Also plotted in these figures is the straight line that extrapolates to $3.0 \mathrm{MA} / \mathrm{ms}$ for the 11.8-MA BPX. Analysis of peak current decay rates in single-null divertor discharges with $\kappa_{x}=1.85$ in DIII-D show $d I_{p} / d t$ proportional to $I_{p}$, extrapolating to $2.7 \mathrm{MA} / \mathrm{ms}$ for BPX. We take the rate of $3.0 \mathrm{MA} / \mathrm{ms}$ to be the maximum peak and average decay rates for which BPX is to be designed.

The Tokamak Simulation Code ${ }^{2}$ (TSC) has been used to simulate disruptive episodes in order to predict the evolution of plasma motion, current decay, induced eddy currents, and forces for the BPX $2.59-\mathrm{m}$ design. The sensitivity of net vacuum vessel forces to $d l_{p} / d l$ and to various initial conditions was investigated to determine the disruption events that produce the most severe loads. Analysis of the evolution of currents, plasma motion, extreme forces, and force distributions leads to the selcction of candidates for the most severe load cases. Several disruption cases, which we characterize as fast radial, fast vertical, slow vertical, etc., are necessary because different cases tend to drive the design of different parts of the vacuum vessel. It must be emphasized that the physics characterization of disruptions is evolving as analysis of experimental data becomes more detailed. This is a priority activity of both the BPX and ITER Physics R\&D programs and can be expected to continue. It is possible that new information from this work will affect the standard BPX disruption guidelines described in this section.

For load analysis of three-dimensional BPX conducting structures, the TSC plasma is represented by approximately 200 filaments with time-varying currents and passed over to the three-dimensional eddy current analysis code SPARK (Ref. 19). SPARK computations with this detailed plasma driver and with finite element representations of the three-dimensional conducting structures yields induced eddy currents and loads suitable for input to the structural analysis codes.

\section{V.H.1. Current Transfer from Plasma to Vessel}

There are two modes for transfer of current between the plasma and conductors such as those that comprise the vacuum vessel, inductive and conductive. The first is due solely to changing poloidal and toroidal flux inside the vessel caused by plasma resistance, inductance, and paramagnetism changes and by plasma motion. The second mode of current transfer involves force-frce flow of current along open field lines in the plasma periphery or "halo" region and directly into conductors that intersect these field lines. The TSC halo model is described in the following section.

\section{V.H.2. TSC Plasma Halo Model}

During disruptions and vertical displacement episodes ${ }^{20}$ (VDEs), there will be large electric fields, large heat flux across the plasma boundary, and gas entering the plasma from the wall. This causes a plasma halo to form in the scrape-off region just outside the main plasma. Damage to conducting structures in PBX-M during disruptive discharges is consistent with large poloidal currents entering the conductors from the plasma scrapeoff layer ${ }^{21}$ (SOL). Recent observations of DIII-D and JET disruptions indicate that up to $20 \%$ of the initial plasma current $I_{p}$ may flow between the SOL and vacuum vessel. An example ${ }^{22}$ is shown in Fig. 5.58, where it can be seen that poloidal SOL current rises to about $0.2 I_{p}$ for DIII-D shot 68668 . Such poloidal SOL currents can lead to localized $J_{\text {pol }} \times B_{t}$ forces that are larger in magnitude than forces from the usual toroidal eddy currents.

Outside the TSC main plasma, there is a region of "halo" plasma in which force-free currents develop and flow along open field lines, intersecting conductors. They then flow along minimum impedance paths and return to the halo plasma. The TSC halo model ${ }^{23}$ also takes into account purely inductive toroidal and poloidal current transfer due to poloidal and toroidal flux changes. 
Jardin et al. MHD EQUILIBRIUM AND STABILITY
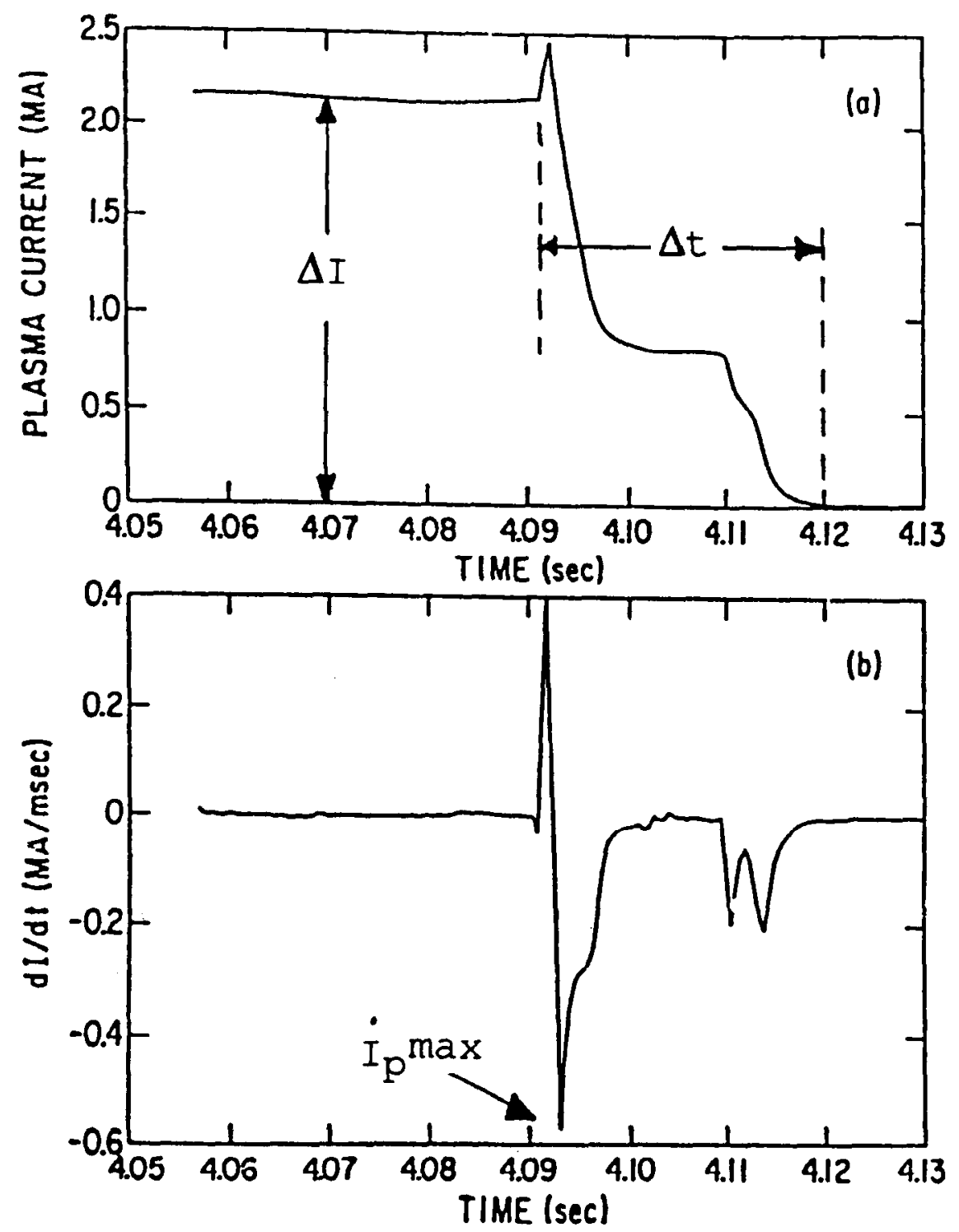

$$
\begin{aligned}
& \text { AVERAge RATE }=\Delta I / \Delta t \\
& \text { PEAK RATE }=\dot{I}_{p} \max
\end{aligned}
$$

Fig. 5.55. Typical traces of plasma current $I_{p}$ versus time and $d I_{p} / d t$ versus time in a TFTR disruption. 


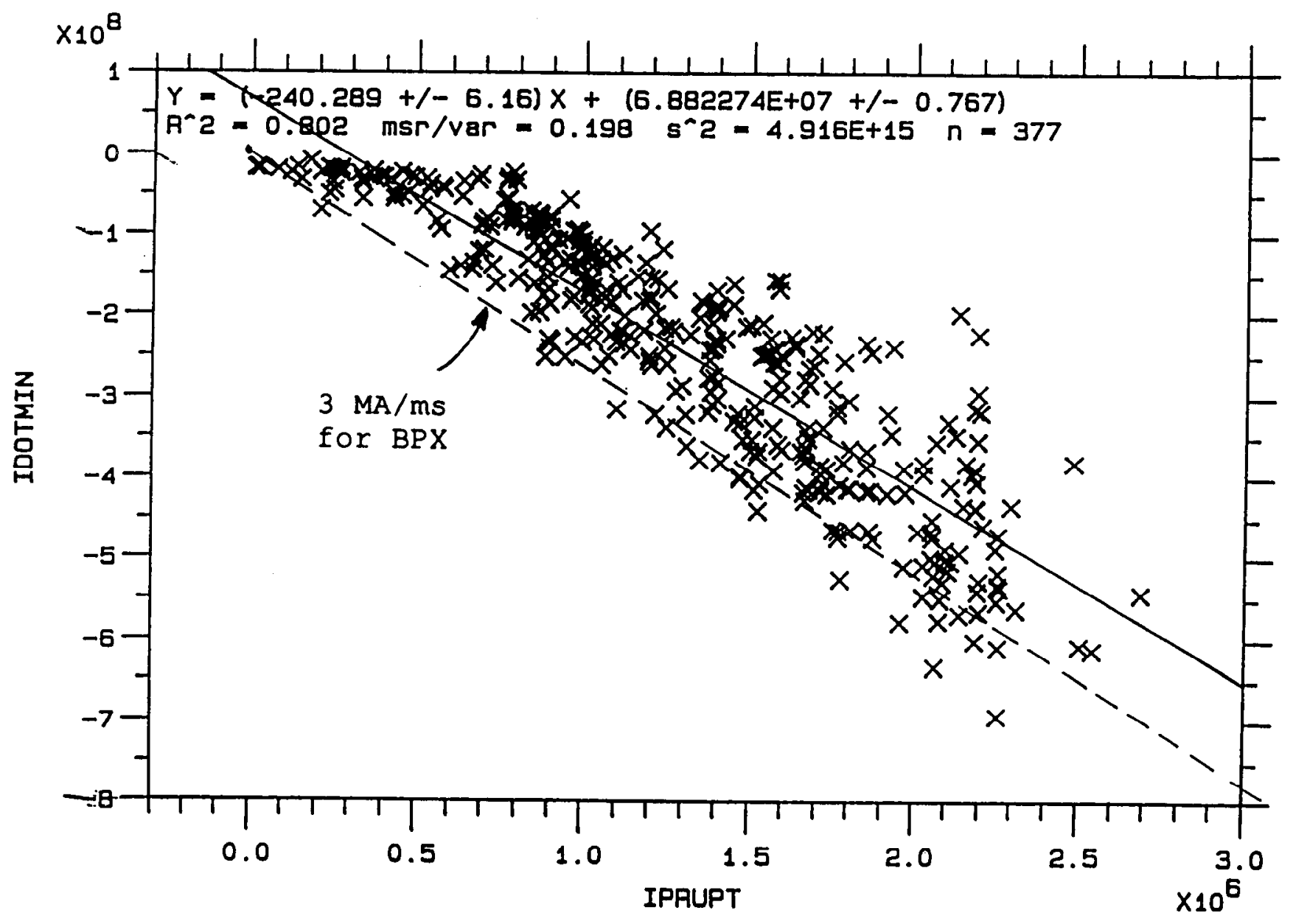

Fig. 5.56. Pcak disruption rates in TFTR plotted versus plasma current prior to the disruption. 
Jardin et al. MHD EQUILIBRIUM AND STABILITY

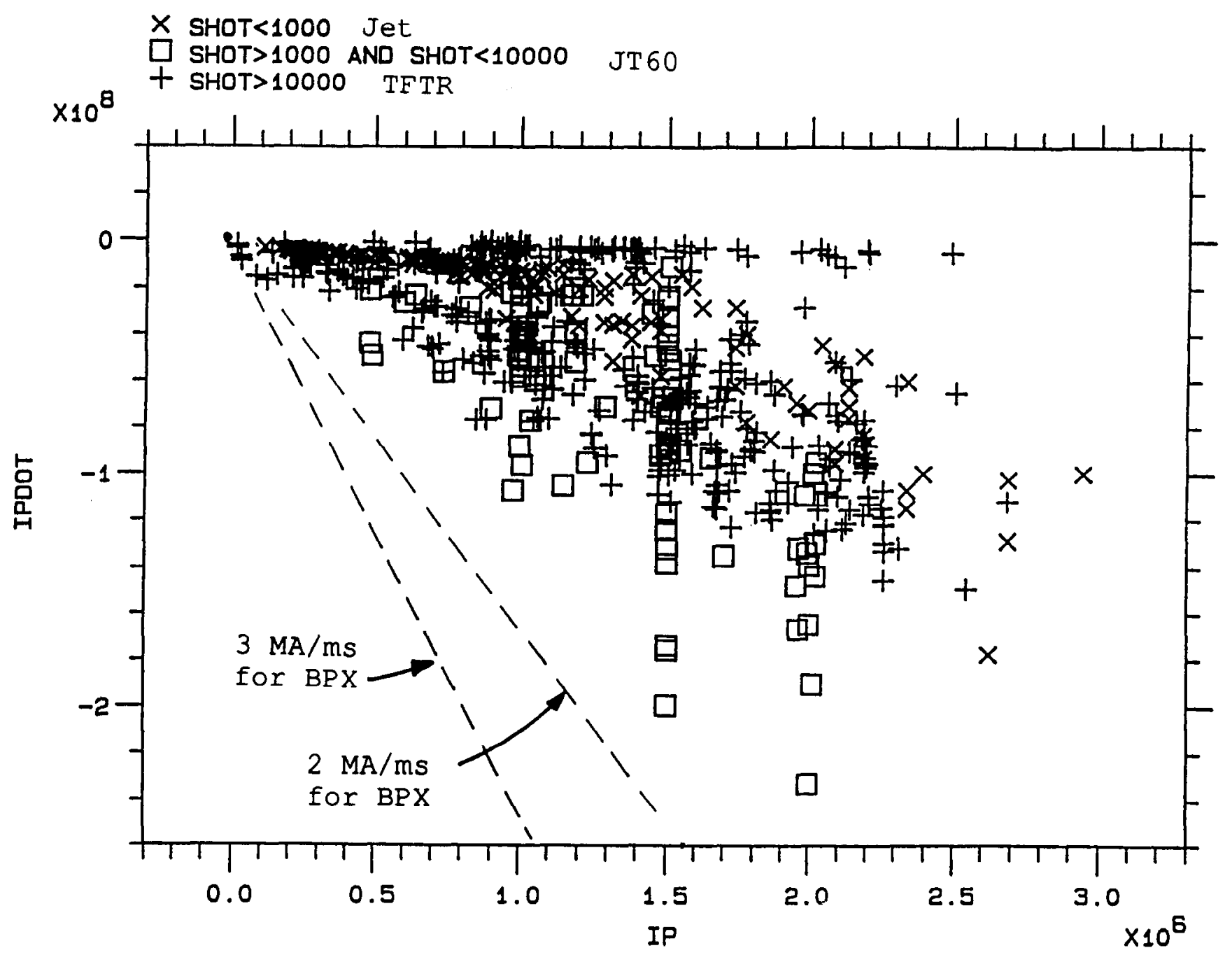

Fig. 5.57. Avcrage disruption rates in JET JT-60 and TFTR plotted versus plasma current prior to the disruption. 

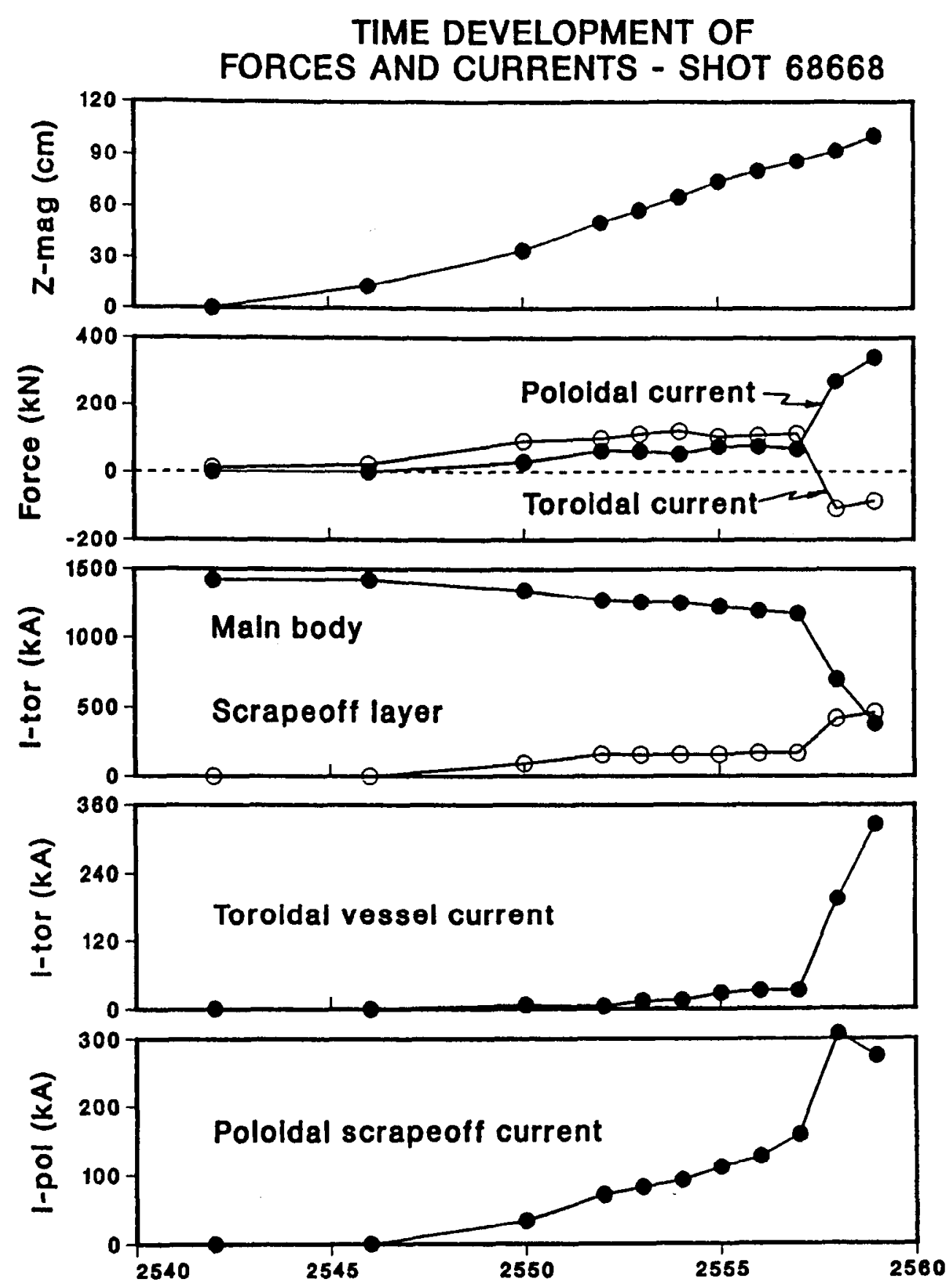

Fig. 5.58. $Z_{\text {mag }}$, forces, toroidal currents, and poloidal scrape-off current for a VDE in DIII-D (taken from Ref. 22). 


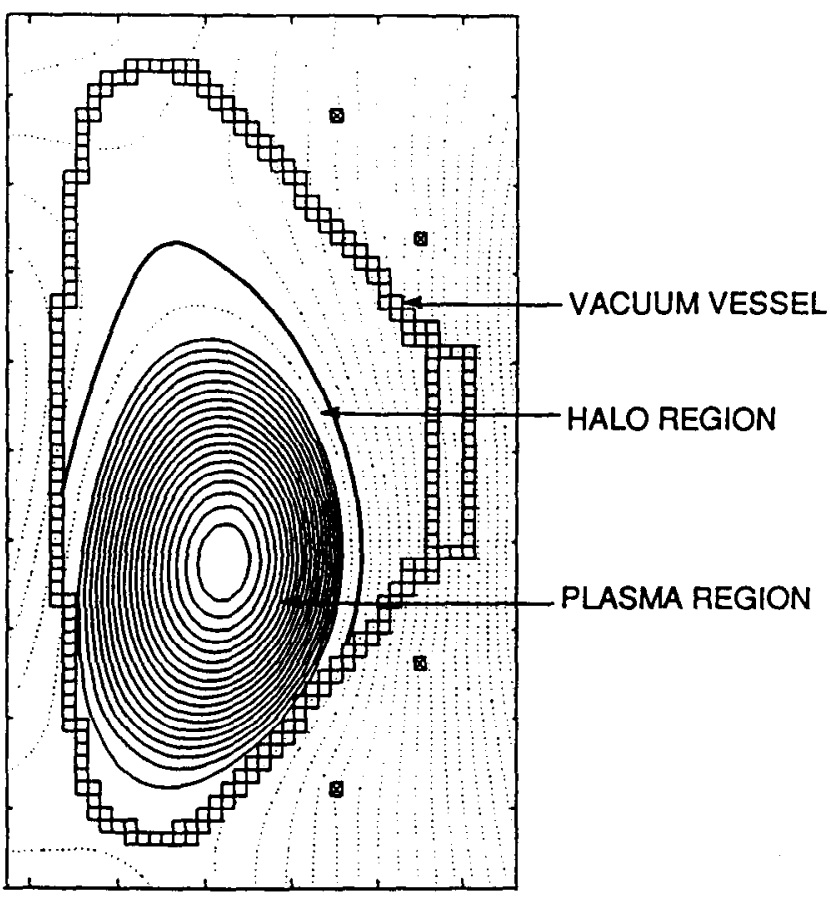

Fig. 5.59. Schematic representation of TSC plasma and halo regions and a BPX vacuum vessel.

The TSC main plasma and plasma halo regions are depicted schematically in Fig. 5.59. The halo is a low-density, low-temperature conducting plasma that exists in regions of open field lines. Toroidal current will be induced in the halo region in the same direction as the plasma toroidal current. The halo current will develop a poloidal component to remain force free. In the halo region,

$$
J \times B=\nabla p=0
$$

and

$$
J_{\text {pol }}=-J_{\text {tor }} B_{\text {pol }} / B_{t} .
$$

Poloidal halo current will flow along open field lines, intersect, and return through the vessel. Both the halo temperature $T_{H}$ and width $W_{H}$ as a fraction of initial plasma poloidal flux $\Psi$ may be specified for TSC calculations. Poloidal resistivities are computed from the halo temperatures by assuming $Z_{\text {eff }}=1$. This halo disruption model has been used ${ }^{24}$ to reproduce both the dynamic plasma behavior and passive stabilizing plate voltages measured during PBX-M disruptions by incorporation of a 3-eV halo.

\section{V.H.3. TSC Models of Torus Conducting Structures}

Conducting structures internal to the TSC computational grid were represented by a series of pas- sive filamentary wires of specified resistivity as discussed in Ref. 24. Figure 5.60 illustrates the filamentary models for the vacuum vessel and IC coils located within the computational grid. Also shown are seven PF coils located outside the TSC computational grid. Circuit equations in TSC permit currents to be induced in the PF coils during the disruption simulations, thereby giving a realistic treatment of the inductive coupling of the vacumm vessel with the $\mathrm{PF}$ coils.

Because the vacuum vessel thickness and composition varied poloidally, several poloidal segments having resistivities in the range 1.2 to $1.6 \times 10^{-6}$ $\Omega \cdot \mathrm{m}$ were used. For each segment, the resistivity was adjusted to give a resistance equal to the resistance of the actual geometric shell. The SPARK code was used to determine the geometry and resistivity for the outboard region of the TSC vacuum vessel model so as to give an axisymmetric approximation of the electromagnetic response of the three-dimensional structure.

The effective toroidal resistance was checked by imposing a constant voltage on the TSC grid and allowing vacuurn vessel current to reach a steadystate value. This procedure yielded a value of 22.8 $\mu \Omega$, in good agreement with the value computed from shell geometry. The poloidal vacuum vessel resistance is $15.7 \mu \Omega$, and the poloidal vacuum vessel inductance is $0.470 \mu \mathrm{H}$. L/R time constants for toroidal and poloidal current flow were 111 and 33 $\mathrm{ms}$, respectively. Independent copper internal control coils $\left(\rho=0.27 \times 10^{-8} \Omega \cdot \mathrm{m}\right)$ were located at $(3.26 \mathrm{~m} \pm 1.89 \mathrm{~m})$ and $(3.75 \mathrm{~m} \pm 1.19 \mathrm{~m})$.

\section{V.H.4. Initial Conditions and Vertical Drift}

Initial PF coil currents for each set of equilibrium conditions $\left(R_{o}, a, \beta_{\text {pol }}, \kappa\right)$ were provided to TSC by the Oak Ridge National Laboratory Fusion Energy Division equilibrium code. ${ }^{25}$ For simulation of VDEs, an initial vertical displacement was introduced and the plasma was then evolved according to the transport equations with the vertical feedback system disconnected. Evolution was continued until $q_{95}$ dropped to the critical value, $q_{\text {crit }}$. For the majority of this work, $q_{\text {crit }}$ was taken to be 2.0 since a $2 / 1$ mode instability would be expected to trigger a disruption. (The exception was for case $220 \mathrm{~B}$ in Table 5.22, where we allowed $q_{\text {crit }}$ to drop to 1.5 in order to assess the effect.) At the time when $q$ reached $q_{\text {crit }}$, the magnetic axis was approximately $62 \mathrm{~cm}$ below midplane. After drift, the thermal quench was initiated by enhancement of the plasma thermal conductivity by a factor between 1000 and 20000 , producing a sudden temperature drop resulting in an enhanced plasma resistivity and a subsequent current quench in the 
Jardin et al. MHD EQUILIBRIUM AND STABILITY

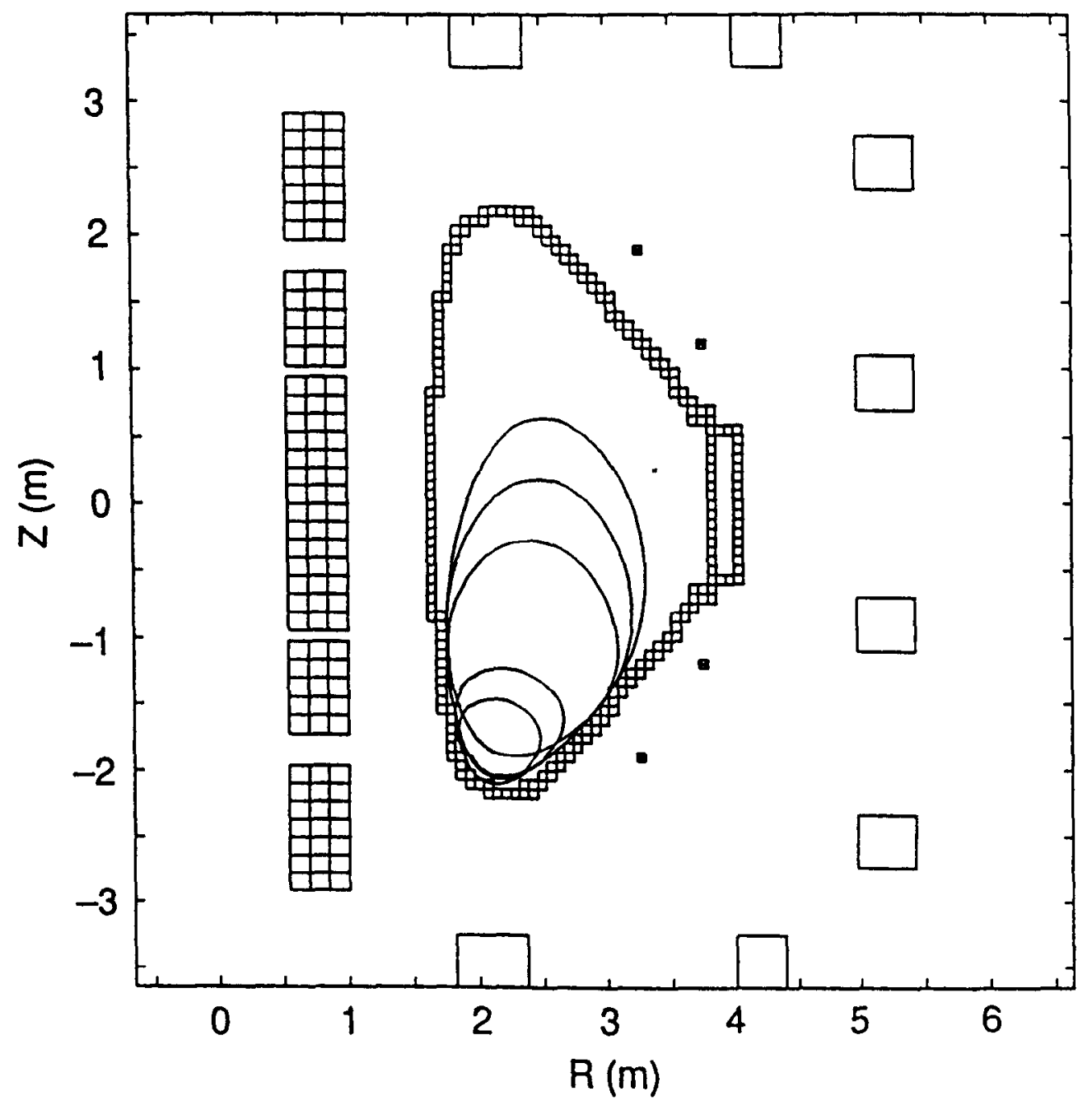

Fig. 5.60. Plasma boundary evolution during a typical TSC VDE simulation for the BPX 2.59-m design. Representations of the vacuum vessel, IC coils, and external PF coils are shown.

Table 5.21. BPX 2.59-m TSC VDE Simulations

\begin{tabular}{|c|c|c|}
\hline Plasma Parameters & Initial Equilibrium & After Drift \\
\hline$q_{95}$ & 3.20 & 2.05 \\
$Z_{\text {mag }}$ & $-0.04 \mathrm{~m}$ & $-0.62 \mathrm{~m}$ \\
$I_{p}$ & $11.8 \mathrm{MA}$ & $11.4 \mathrm{MA}$ \\
$B_{\text {tor }}$ & $9.0 \mathrm{~T}$ & $9.0 \mathrm{~T}$ \\
$R_{o}$ & $2.59 \mathrm{~m}$ & $2.54 \mathrm{~m}$ \\
$a$ & $0.79 \mathrm{~m}$ & $0.75 \mathrm{~m}$ \\
$\kappa_{95}$ & 1.96 & 1.62 \\
$\delta_{95}$ & 0.33 & 0.15 \\
$\ell_{i} / 2$ & 0.40 & 0.35 \\
$\beta$ & $2.1 \%$ & $2.4 \%$ \\
$\beta_{\text {pol }}$ & 0.36 & 0.33 \\
\hline
\end{tabular}




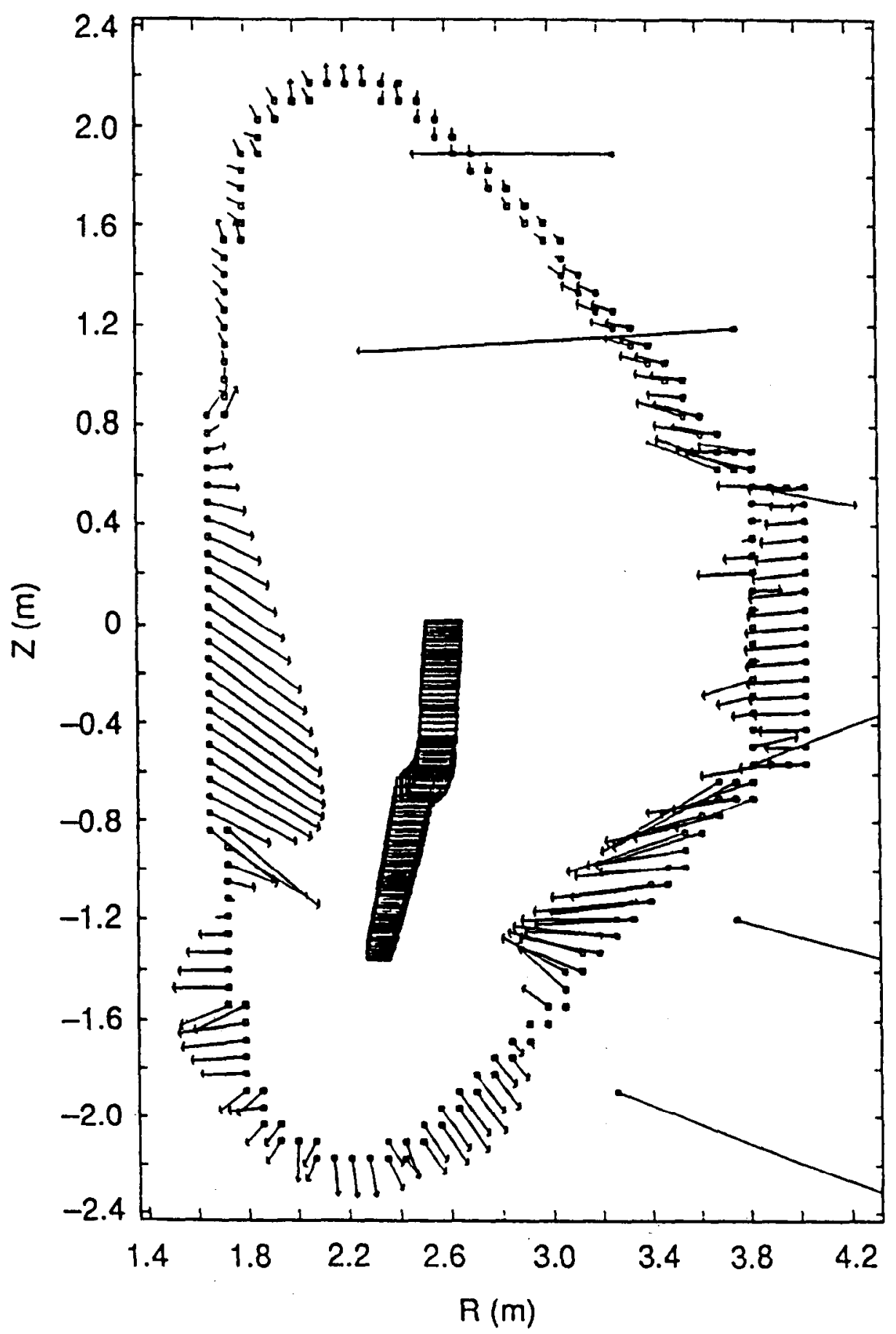

Fig. 5.61. Snapshot of force distribution $\left(I_{p}=4.9 \mathrm{MA}\right)$ for the BPX 2.59-m vacuum vessel for a VDE with $\left\langle d I_{p} / d t\right\rangle=-3.0 \mathrm{MA} / \mathrm{ms}$. Forces include contributions from poloidal and toroidal currents. Boxes with area proportional to $I_{p}$ are plotted along the plasma trajectory. Initially, $I_{p}=11.8 \mathrm{MA}, \beta=2.1 \%$, and $\beta_{\text {pol }}=0.36$. 
range of -0.3 to $-3.0 \mathrm{MA} / \mathrm{ms}$.

Thermal and current quench phases were simulated for several values of $\left\langle T_{H}\right\rangle$ ranging from 0.2 to $4.5 \mathrm{eV}$. In the halo region, the temperature is assumed linear in $\Psi$ from the plasma-halo boundary to the halo-vacuum boundary.

After thermal quench, the plasma vertical motion accelerates until the plasma is subject to restriction by a set of limiters near the first wall and divertor locations. Motion of the plasma boundary during a disruption is illustrated in Fig. 5.60, which shows overlays of plasma-halo boundaries for a typical VDE simulation. Table 5.21 contains values of selected initial plasma parameters for the initial equilibrium and after vertical drift.

\section{V.H.5. Results}

Disruption scenarios were simulated for $\left\langle d I_{p} / d t\right\rangle$ in the range -0.3 to $-3.0 \mathrm{MA} / \mathrm{ms}$. Figure 5.61 is a snapshot of the force distribution $\left(I_{p}=4.9\right.$ MA) for a VDE simulation with $\left\langle d I_{p} / d t\right\rangle=$ $3.0 \mathrm{MA} / \mathrm{ms}$. Forces include contributions from poloidal and toroidal currents. Boxes with area proportional to $I_{p}$ are plotted along the plasma trajectory. Initially, $I_{p}=11.8 \mathrm{MA}, \beta=2.1 \%$ and $\beta_{\text {pol }}=0.36$.

A snapshot of BPX vessel poloidal current versus poloidal angle for a VDE simulation with $\left\langle d I_{p} / d l\right\rangle=-0.6 \mathrm{MA} / \mathrm{ms}$ is presented in Fig. 5.62. Initially, $I_{p}=11.8 \mathrm{MA}, \beta=2.1 \%, \beta_{\text {pol }}=0.36$, $R_{o}=2.59 \mathrm{~m}, a=0.79 \mathrm{~m}$, and $\kappa_{95}=1.93$. The peak near 270 deg represents poloidal current that transfers from the halo region to the vessel at the points of contact at poloidal angles near $225 \mathrm{deg}$ and $300 \mathrm{deg}$. Without this effect, the poloidal current would be independent of poloidal angle. For the time instant displayed, the induced poloidal current is nearly zero.

Figure 5.63 displays plasma current $I_{p}$, vacuum vessel toroidal current $I_{v v}$ (TOR), and vacuum vessel conductive halo current $I_{H}$ versus time for a simulation with $\left\langle d I_{p} / d t\right\rangle=-0.6 \mathrm{MA} / \mathrm{ms}$ and $\left\langle T_{H}\right\rangle_{\text {quench }}=4.5 \mathrm{eV}$. It should be noted that most of the buildup of $I_{H}$ occurs in the last third of the drift phase $\left(\left\langle T_{H}\right\rangle_{d r i f t}=20 \mathrm{eV}\right)$ when the plasma halo touches the vacuum vessel bottom.

The peak $I_{H} / I_{p}$ is plotted versus $\left\langle T_{H}\right\rangle_{\text {quench }}$ in Fig. 5.64. Except where noted, $W_{H}=0.2 \Psi_{\text {Plasma }}$ and $\left\langle T_{H}\right\rangle_{\text {drift }}=20 \mathrm{eV}$ during the vertical drift phase. As expected, higher halo temperatures during drift and disruption phases and greater halo extent both lead to greater conductive vacuum vessel poloidal current flow.

Another halo effect is depicted in Fig. 5.65, which shows that both peak vertical velocity $d z / d t$ and mean plasma current decay rate $\left\langle d I_{p} / d t\right\rangle$ decrease

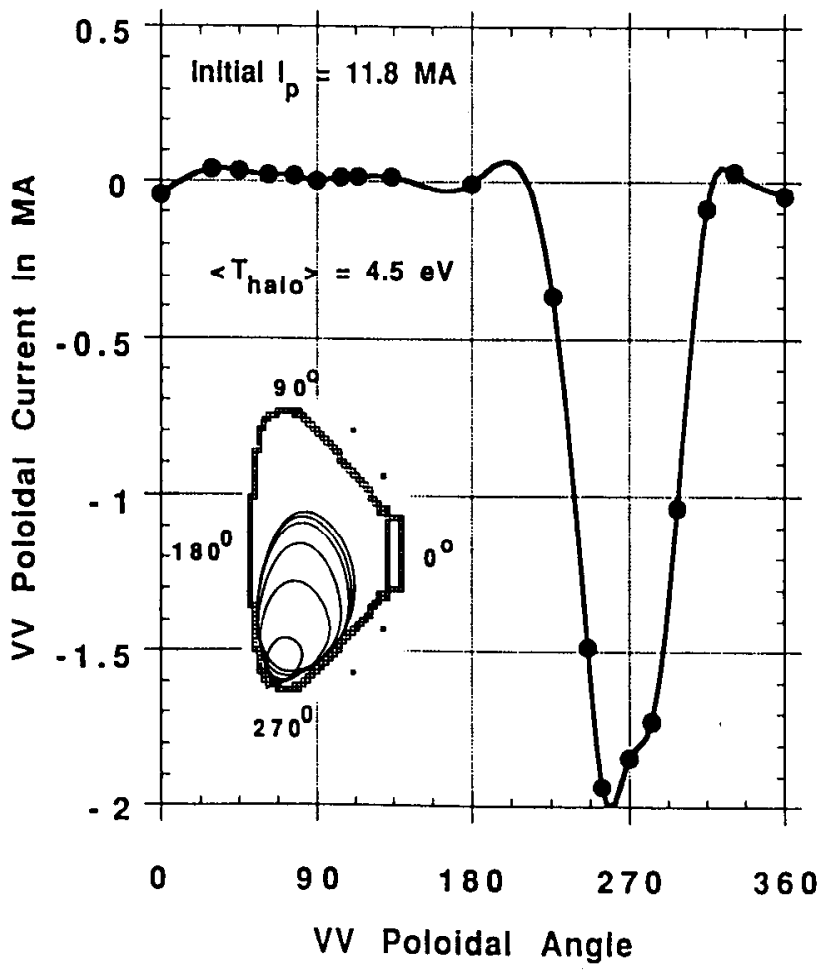

Fig. 5.62. Snapshot of vessel poloidal current versus poloidal angle for a BPX VDE with $\left\langle d I_{p} / d t\right\rangle=$ $-0.6 \mathrm{MA} / \mathrm{ms}$. Initially, $I_{p}=11.8 \mathrm{MA}, \beta=2.1 \%$, $\beta_{\text {pol }}=0.36, R_{o}=2.59 \mathrm{~m}, a=0.79 \mathrm{~m}$, and $\kappa_{95}=1.93$. The difference between top and bottom currents is due to conductive poloidal current flow between plasma and vessel.

rapidly as $\left\langle T_{H}\right\rangle$ increases. For a given $\left\langle d I_{p} / d t\right\rangle$, the net vertical vessel load evolution is modified significantly by the poloidal current contributions, as shown in Fig. 5.66. Furthermore, plasma halos change the load and pressure distributions markedly, even though the net vertical and radial vacuum vessel forces are relatively insensitive to the presence of the halo. This is illustrated in Fig. 5.67 by plots of maximum pressure on vacuum vessel bottom and inboard walls versus $\left\langle d I_{p} / d t\right\rangle$. A summary of extreme forces and pressures for the VDE simulations discussed here is presented in Table 5.22 .

\section{V.H.6. Summary}

Vertically moving disrupting plasmas both with and without a surrounding halo were simulated for the BPX 2.59-m design for a range of $T_{H}$ and $W_{H}$. The initial conditions $I_{p}=11.8 \mathrm{MA}, \beta=2.1 \%$, and 
Jardin et al. MHD EQUILIBRIUM AND STABILITY

Table 5.22. BPX 2.59-m TSC Disruption Simulations with Initial $I_{p}=11.8 \mathrm{MA}, \beta=2.1 \%$, and $\beta_{\text {pol }}=0.36$.

\begin{tabular}{|l|r|r|r|r|r|r|r|r|l|}
\hline Case & $904 \mathrm{D}$ & $906 \mathrm{C}$ & $912 \mathrm{D}$ & $911 \mathrm{~A}$ & $1005 \mathrm{D}$ & $1031 \mathrm{C}$ & $220 \mathrm{~B}$ & $212 \mathrm{D}$ & \\
\hline$Z_{\text {mag }}$ after drift & 0.6 & 0.6 & 0.6 & 0.6 & 0.6 & 0.0 & 0.84 & 0.6 & $\mathrm{~m}$ \\
Halo Extent & None & $20 \%$ & $20 \%$ & $20 \%$ & $40 \%$ & None & $40 \%$ & None & \\
Quench $T_{H}$ & 0 & 1.5 & 0.2 & 4.5 & 4.5 & - & 4.5 & - & $\mathrm{eV}$ \\
$d I_{p} / d t$ & 3.0 & 1.5 & 3.0 & 0.5 & 0.3 & 3.0 & 0.35 & 0.35 & $\mathrm{MA} / \mathrm{ms}$ \\
Peak I(Halo) & 0 & 1.4 & 1.4 & 1.7 & 1.9 & 0.1 & 2.2 & - & $\mathrm{MA}$ \\
$F_{z}$ (vacuum vessel) & 3.7 & 4.0 & 4.1 & 5.0 & 5.2 & 0 & 6.6 & 5.6 & $\mathrm{MN} / \mathrm{rad}$ \\
$F_{r}$ (vacuum vessel) & 12.9 & & 12.7 & 11.6 & 10.8 & 13.1 & 10.6 & 12.0 & $\mathrm{MN} / \mathrm{rad}$ \\
Maximum Inboard Pressure & 3.5 & 3.2 & 3.5 & 2.8 & 2.5 & 3.1 & 2.8 & 2.9 & $\mathrm{MPa}$ \\
Maximum Bottom Pressure & 0.7 & 1.0 & 0.7 & 1.3 & 1.4 & 0.5 & 1.6 & 1.5 & $\mathrm{MPa}$ \\
\hline
\end{tabular}

BPX $2.59 \mathrm{~m} \quad 0.5 \mathrm{MA} / \mathrm{ms}$ Vertical Disruption

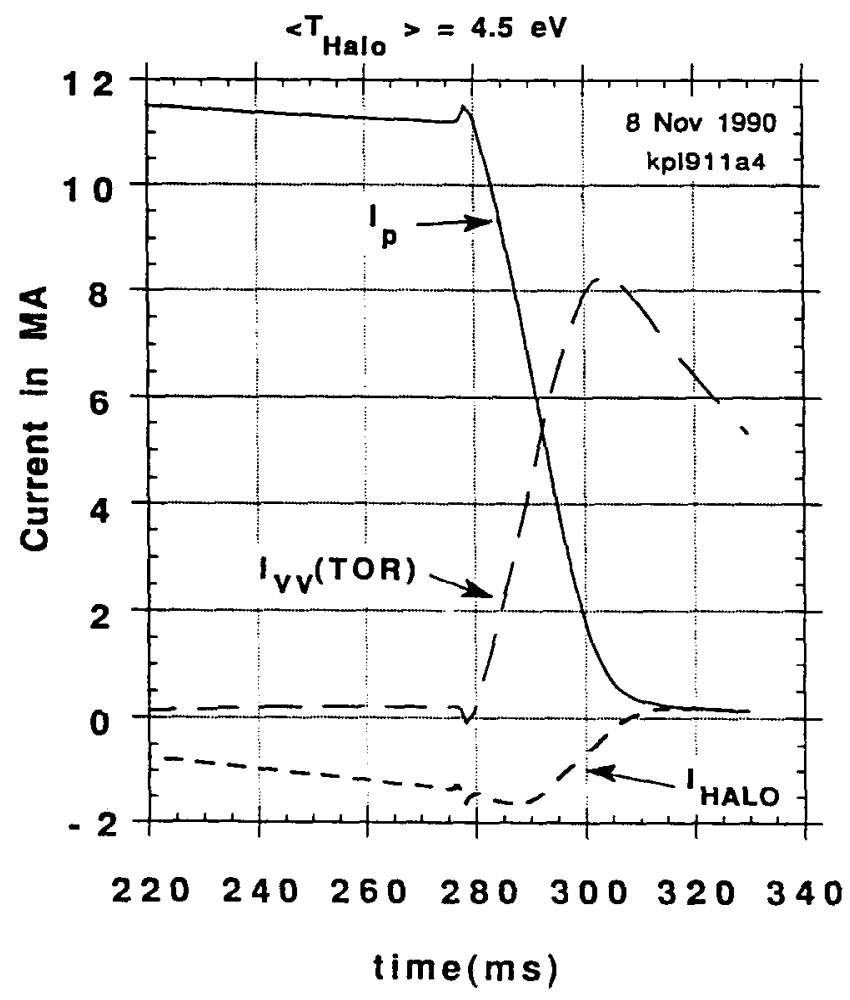

Fig. 5.63. Plasma current $I_{p}$, vacuum vessel toroidal current $I_{V V}(\mathrm{TOR})$, and vacuum vessel conductive halo current $I_{H}$ versus time for a simulation with $\left\langle d I_{p} / d t\right\rangle=-0.6 \mathrm{MA} / \mathrm{ms}$ and $\left\langle T_{H}\right\rangle_{\text {quench }}=4.5 \mathrm{eV}$.
BPX 2.59 m TSC Vertical Halo Disruptions

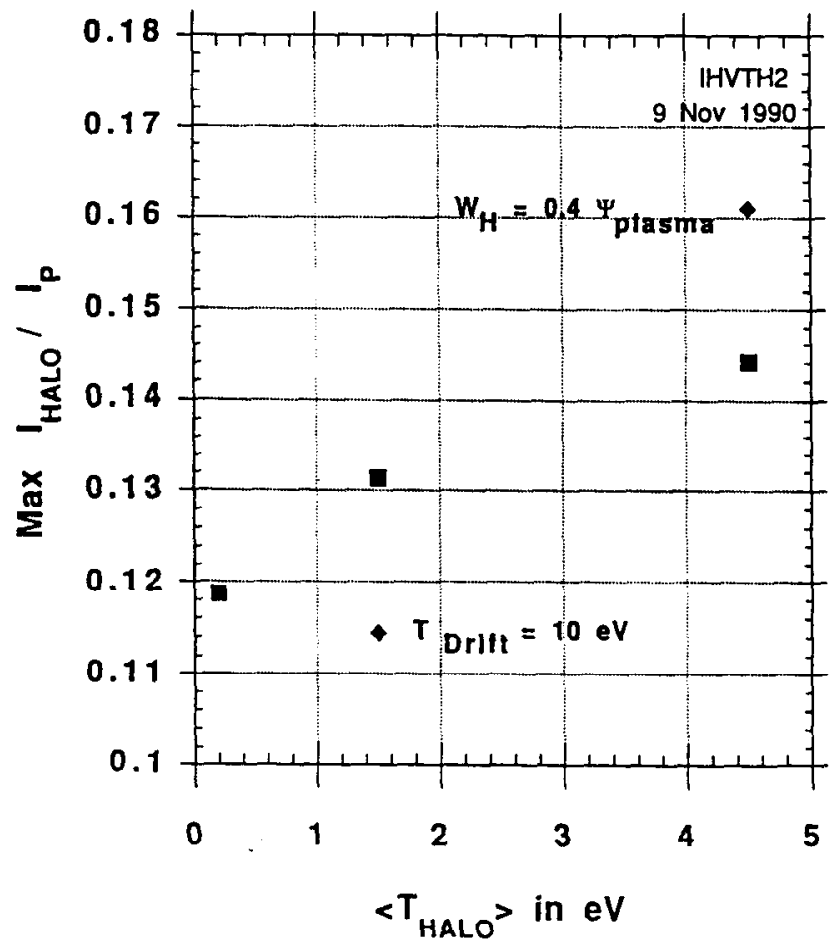

Fig. 5.64. Peak $I_{H} / I_{p}$ versus $\left\langle T_{H}\right\rangle_{q u e n c h}$. Except where noted, $W_{H}=0.2 \Psi_{\text {plasmas }}$ and $\left\langle T_{H}\right\rangle_{\text {drift }}=20$ $\mathrm{eV}$ during the vertical drift phase.

$\beta_{\text {pol }}=0.36$ were used for all simulations. Prior to thermal quench, the plasma was allowed to drift vertically until $q_{95}=2.0\left(Z_{\operatorname{mag}}=-0.62 \mathrm{~m}\right)$, or until $q_{95}=1.5\left(Z_{\text {mag }}=-0.84\right)$. The present sim- 
BPX $2.59 \mathrm{~m}$ TSC Vertical Disruptions

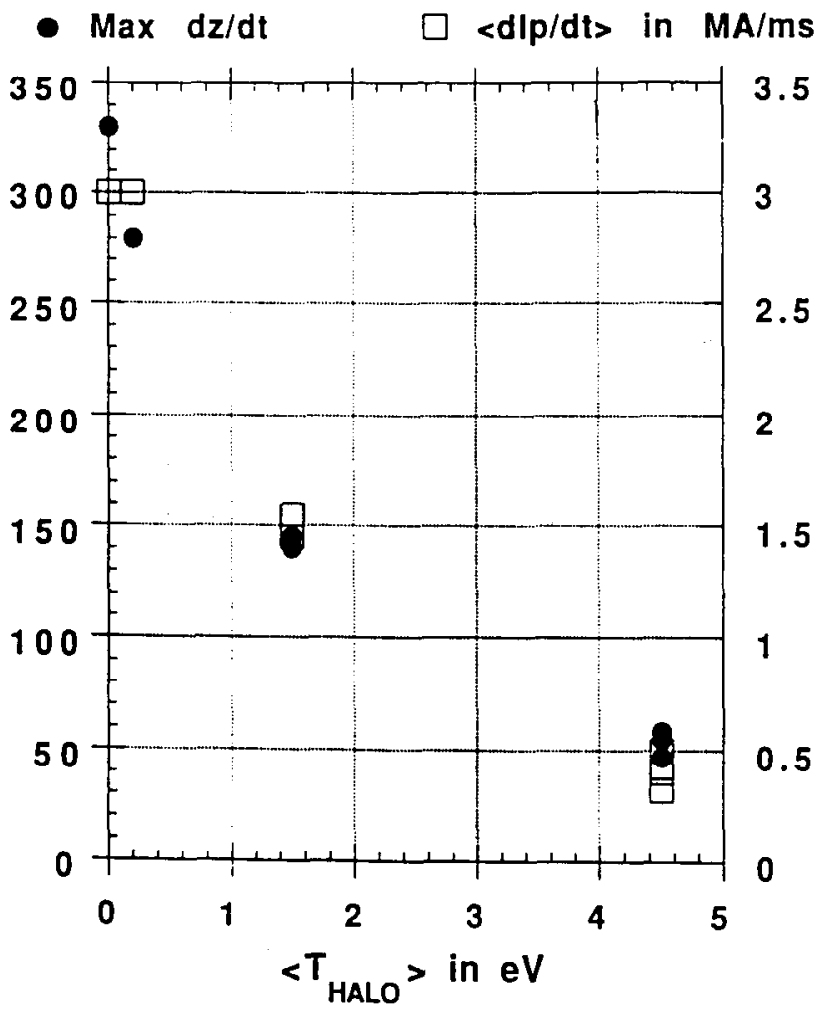

Fig. 5.65. Peak vertical velocity $d z / d t$ and mean plasma current decay rate $\left\langle d I_{p} / d t\right\rangle$ versus $\left\langle T_{H}\right\rangle_{\text {quench }}$ for BPX 2.59-m vertical disruptions.

ulations, which our analysis and the experimental data base to date tell us are worst-case events, lead to several conclusions and observations:

1. A plasma halo retards vertical motion and current decay. Maximum vertical velocity was reduced from the no-halo value of $330 \mathrm{~m} / \mathrm{s}$ to 150 $\mathrm{m} / \mathrm{s}$ for $\left\langle T_{H}\right\rangle_{\text {quench }}=1.5 \mathrm{eV}$ and to $50 \mathrm{~m} / \mathrm{s}$ for $\left\langle T_{H}\right\rangle_{\text {quench }}=4.5 \mathrm{eV}$.

2. The predicted peak vacuum vessel poloidal conductive currents (up to $0.19 I_{p}$ ) are consistent with measured DIII-D values of approximately $0.2 I_{p}$.

3. Pcak vacuum vessel inboard pressure of 3.5 $\mathrm{MPa}$ occurs for the fastest current decay (3.0 $\mathrm{MA} / \mathrm{ms}$ ), whereas peak bottom pressure occurs for the slowest current decay (highest $T_{H}$ ). Large localized forces arise from halo-to-vessel currents. For example, maximum (compressive) vertical forces on the vessel bottom (near the decaying plasma) are up to three times larger when halo currents are included; however, the net vertical and radial vacuum vessel

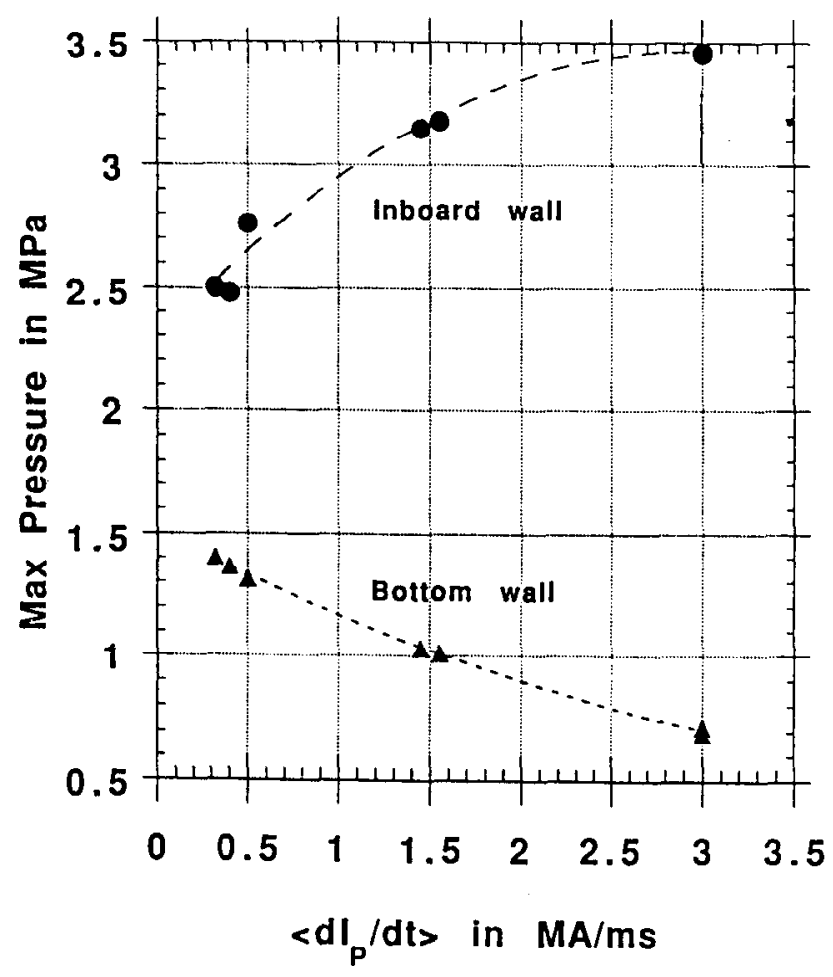

Fig. 5.66. Evolution of net vertical forces for the BPX 2.59-m vacuum vessel for a VDE with $\left\langle d I_{p} / d t\right\rangle=-0.5 \mathrm{MA} / \mathrm{ms}$ and $\left\langle T_{H}\right\rangle_{q u e n c h}=4.5 \mathrm{eV}$, $F_{z}(p o l)=J_{p o l} \times B_{\text {tor }}$ and $F_{z}($ tor $)=J_{\text {tor }} \times B_{\text {pol }}$. Initially, $I_{p}=11.8 \mathrm{MA}, \beta=2.1 \%$, and $\beta_{p o l}=0.36$.

forces are relatively insensitive to the presence of the halo.

4. For the vacuum vessel bottom, the extreme values of $F_{z}$ (halo) and pressure increase with increasing $T_{H}$ during quench for values of $0<$ $T_{H}<4.5 \mathrm{eV}$.

5. Simulations with different initial $\beta_{\text {pol }}$ and $Z_{\text {mag }}$ have been observed to alter the relative importance of poloidal and toroidal current effects, but not to change the overall forces significantly.

6. Extreme vessel radial forces are not highly sensitive to $d I_{p} / d t$, but the magnitude of the cxtreme net vertical force is $25 \%$ larger for the slowest current decay than for the fastest current decay. Slower plasma current decay rates give more severe net vertical loads because the plasma current decay occurs when the plasma has moved farther off-midplane than is the case for faster decay rates. 


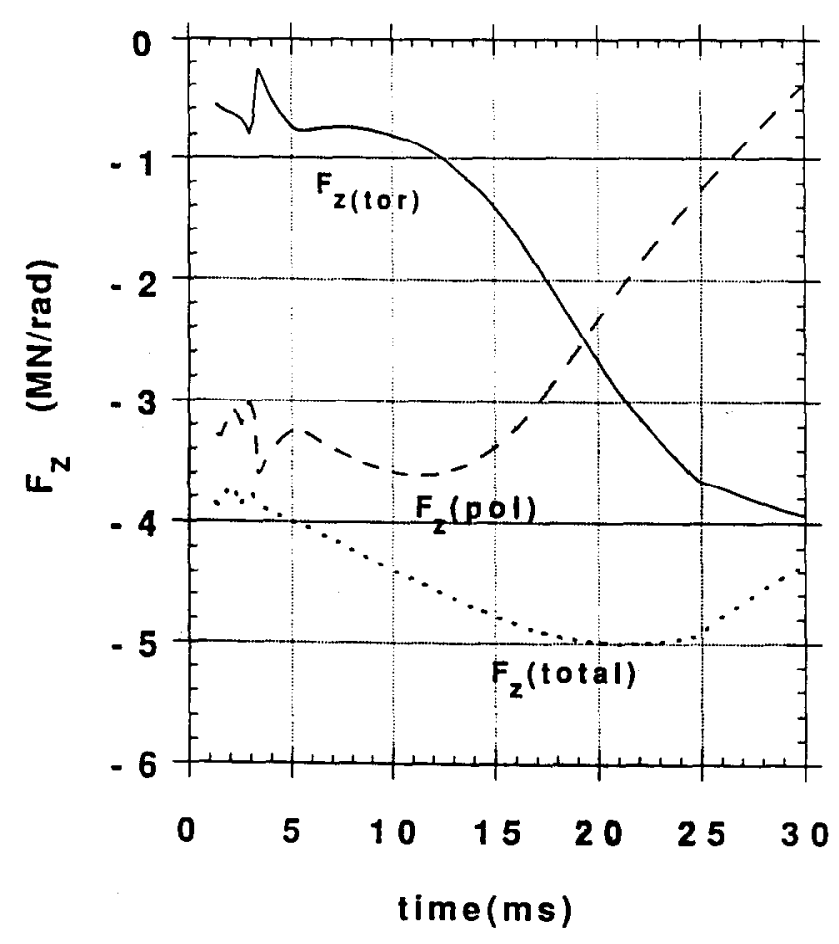

Fig. 5.67. Maximum pressure on vacuum vessel bottom and inboard walls versus $\left\langle d I_{p} / d t\right\rangle$ for BPX 2.59-m VDE simulations.

Because the halo retards both plasma motion and current quench rate, higher $T_{H}$ values give larger extreme vacuum vessel halo currents and larger localized $J_{\text {pol }} \times B_{\text {tor }}$ forces in the vacuum vessel region where halo currents flow. For the cases considered here, the slowest quench rate, 0.3 $\mathrm{MA} / \mathrm{ms}$, corresponding to $\left\langle T_{H}\right\rangle_{\text {quench }}=4.5 \mathrm{eV}$, gave the largest bottom wall pressure of $1.4 \mathrm{MPa}$. Inspection of Fig. 5.67 shows that higher $T_{H}$, i.e., slower current quench rate, would give at most only about 1.6 $\mathrm{MPa}$ for bottom wall pressure.

Detailed structural analysis of the vessel response to the loads produced by these disruptions is still in progress. The stresses in the critical inboard wall region are within allowable limits for those cases that have the maximum loads there. In other regions, it is expected the design can be adjusted if necessary to withstand the disruption loads. The sizing of the divertor and first-wall tiles and their supports has been driven by disruption eddy and halo current loads. Engineering analysis for a disruption case with maximum current decay rate and one with the maximum halo current show that the tiles will structurally be able to withstand disruptions.

\section{V.H.7. Future Work}

Further calculations will be done of disruptive discharges from DIII-D and JET bccause of their relevance to BPX design plasmas. The goals are to reproduce the observed plasma current decay, trajectory evolution, and poloidal vacuum vessel current flow. In addition, halo model refinements may be indicated by detailed comparison with experiment. Sensitivities of simulation predictions to halo extent and temperature and to initial conditions will also be studied.

Analysis of DIII-D and JET observations is consistent with SOL temperatures in the range 10 to $20 \mathrm{eV}$ during VDEs. However, there are large uncertainties in SOL resistivity estimates because $Z_{\text {eff }}$ is known poorly. Direct measurement of SOL temperature, extent, and $Z_{\text {eff }}$ would lead to significant improvement in the characterization of VDEs and the concomitant effects on tokamak structures.

\section{V.I. OTHER MHD STABIUTY RESULTS}

It has long been recognized that MHD stability considerations are an important part of device design. The plasma parameters significant to MHD stability are the plasma geometry, safety factor at the plasma edge, toroidal $\beta, I / a B$ and the shapes of the plasma pressure and current profiles. In the current BPX design, these are given in Table 5.23.

Table 5.23. Plasma Parameters Used in MHD Stability Computation

$$
\begin{array}{ll}
R=2.59 \mathrm{~m} & q_{95} \geq 3.2-3.5 \\
a=0.79 \mathrm{~m} & I / a B=1.66 \mathrm{MA} / \mathrm{mT} \\
R / a=3.2, & 2.0 \leq p_{o} /\langle p\rangle \leq 4.2 \\
\kappa_{95} \leq 2.0 & \ell_{i} \approx 0.8 \\
0.2<\delta_{95}<0.4 & \beta_{\text {Total }} \leq 2.75 \%, \\
I=11.8 \mathrm{MA} & g \leq 1.7 \\
B=9 \mathrm{~T} & \beta_{p} \leq 0.5
\end{array}
$$

The empirical Troyon limit, ${ }^{26}$ which represents the beta value above which disruptions set in or confinement deteriorates dramatically in most tokamak experiments, is well known to have an approximate correlation with the beta threshold for the onset of ideal MHD instabilities. This limit is commonly expressed as

$$
\beta_{\max } \leq g \frac{I(\mathrm{MA})}{a(\mathrm{~m}) B(\mathrm{~T})}
$$


with $2.5 \leq g \leq 3.5$. The range in $g$ is due to the relative importance given to ideal kink and high- $n$ ballooning instabilities. It is equally well established that the Troyon limit represents an optimal situation and is frequently not achieved. The principal cause for the limitations are related to (a) the total plasma current exceeding a limit set by $q_{\text {edge, }}$ (b) the shape of the plasma cross section, and (c) the detailed shapes of the plasma current and pressure profiles. Of these three factors, the total plasma current represents the most difficult obstacle. When the plasma current $I$ is such that $q_{\text {edge }} \leq 2$, the discharge is almost certain to disrupt. These disruptions are primarily due to current-driven external kink modes and are not related to beta effects. Operation at $2 \leq q_{\text {edge }} \leq 3$ requires great care to avoid frequent disruptions; however, when $q_{\text {edge }}>3$, disruptions can generally be avoided. In BPX, the current is restricted so that $q_{\text {edge }} \geq 3.2$, reaching 3.5 at the highest betas achieved at the end of the discharge. The condition, $q_{\text {edge }}>3.2$, however, is not sufficient to guarantee stability. We must still consider stability to pressure-driven modes, i.e., high- $n$ ballooning modes as well as pressure-driven kink modes. It is difficult to consider these independently of the role of the plasma cross section, particularly since $\mathrm{BPX}$ is designed to have a rather large elongation, $\kappa_{95} \approx 2$. While $\kappa$ is relatively high, DIII-D and JET have shown that it is possible to safely operate in this regime. ${ }^{19,20}$ However, theoretical analysis indicates that the triangularity $\delta_{95}$ plays a critical role in maintaining stability to ideal MHD modes. The most sensitive instability is the internal kink mode. ${ }^{21}$ This result is indicated in Fig. 5.68, which shows the dependence of the beta limit, expressed in terms of $\beta_{\text {pol }}$, as a function of $\kappa_{95}$ for several values of the triangularity, $\delta_{95}=0.1,0.3,0.5$, and 0.7 . It should be noted here that this study was for an earlier BPX design point and should only be used qualitatively for the current design. In principle, the greater the triangularity, the better the stability properties. However, this has to be offset by the limitations imposed by the PF system, which has to shape the plasma, as well as the requirement to dissipate the heat load on the divertor. The design value, $0.25<\delta_{95}<0.4$, represents the best compromise when all these factors are included. The effect of these shape factors on the external kink and ballooning modes is less pronounced and is shown in Fig. 5.69. Note that these results are for optimized pressure profiles that generally raises the beta limit so that $g>3$.

The role of the plasma profiles, current and pressure, is the most difficult to address comprehensively. We adopt three approaches and use profiles that are (a) transport-consistent, (b) analytically

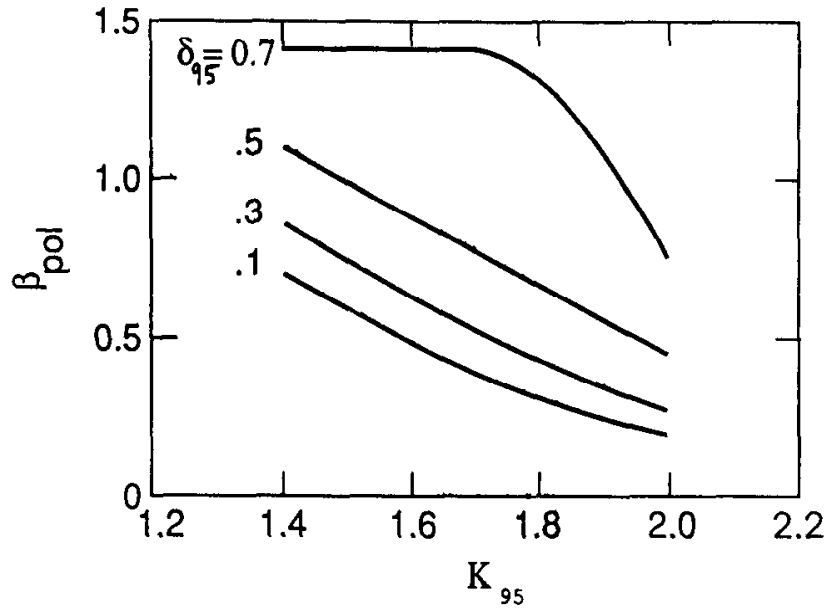

Fig. 5.68. Internal kink stability limits in terms of $\beta_{\text {pol }}$ as a function of elongation for fixed triangularity in an earlier BPX design.

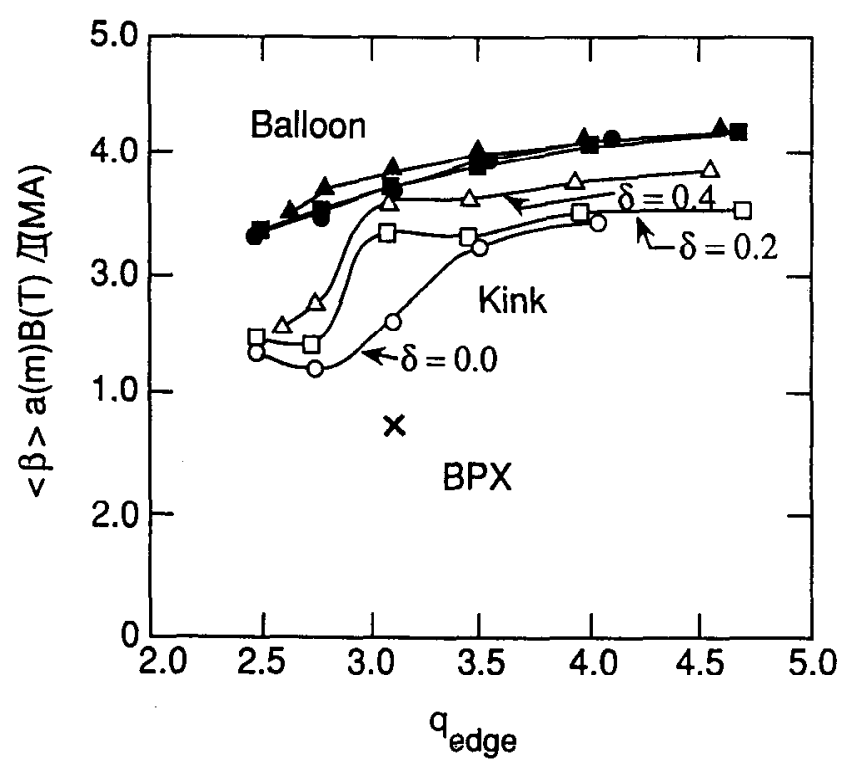

Fig. 5.69. Marginal stability boundaries for $n=\infty$ ballooning and $n=1$ kink modes at fixed values of triangularity, plotted as $\beta a B / I$ versus $q_{\text {edge }}$ for $q_{\text {aris }} \approx 1.04$ and $R=2.11, a=0.65, \kappa=1.91$. The pressure profiles were optimized to yield the highest beta threshold for ballooning modes.

prescribed, and (c) numerically optimized. Many detailed studies of these three classes of profiles have been undertaken and are described in Ref. 28. Here we present the essential features from that report that are relevant to the current design, as well as the main results of subsequent studies.

We quote the results of analysis of a TSC fiducial discharge ${ }^{28}$ at different time slices as beta evolved to its final value of $\approx 2.8 \%$. This fiducial run was 


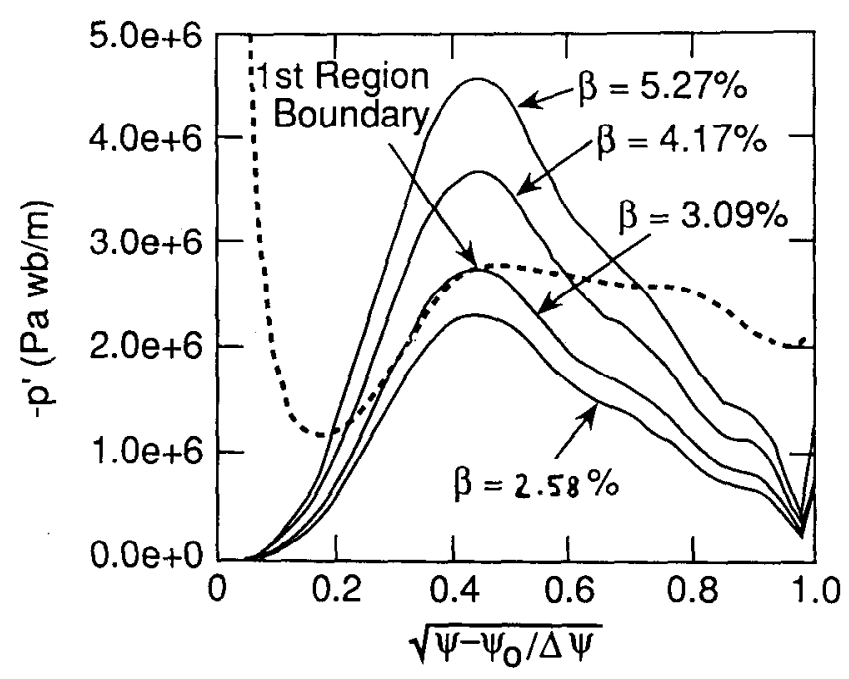

Fig. 5.70. Comparison of the stability boundary for ballooning modes with the pressure profiles for a fiducial discharge at several values of beta.

for an earlier design point with $R=2.1 \mathrm{~m}$. However, the results are still valid since the stability depends on the aspect ratio, which remains unchanged, rather than the major radius. The stability analysis shows that for $\beta \geq 2 \%$, the plasma is marginally unstable to an internal kink mode and is stable to ballooning modes. A slight flattening of the pressure profile near the axis completely stabilizes the internal kink and permits us to determine the threshold for ballooning modes. This is shown in Fig. 5.70, which shows the $p^{\prime}$ profile as a function of the radius for three different values of $\beta$, as well as the stability boundary to ballooning modes. The marginal $\beta \approx 3 \%$ is considerably below the Troyon limit $(\approx 5.5 \%)$ and is only slightly larger than the operational value of $2.75 \%$.

This relatively low threshold for instability is related to the rather peaked nature of the pressure profile found in the simulation. We study this relationship between the peaking factor, $P P F$ $\left(\equiv p_{o} /\langle p\rangle\right)$ and the stability boundary by considering a family of equilibria where the pressure profile is specified as an analytic function. The $P P F$ is varied over a wide range to span the predictions of different transport models and different operating regimes, $2.0 \leq P P F \leq 4.2$. The threshold for instability is plotted in Fig. 5.71. The results indicate that the Troyon limit of $6 \%$ is achieved when $P P F \approx 2.5$ and rapidly drops as the $P P F$ is increased and reaches $\approx 2.8 \%$, when $P P F \approx 4.2$. This implies that if the experimental BPX profile is in the middle of the range so that $P P F \approx 3.5$, then the operational beta is at least $25 \%$ below the stability limit. If $P P F$ is larger and at the limit of the predicted range, then it is at marginal stability

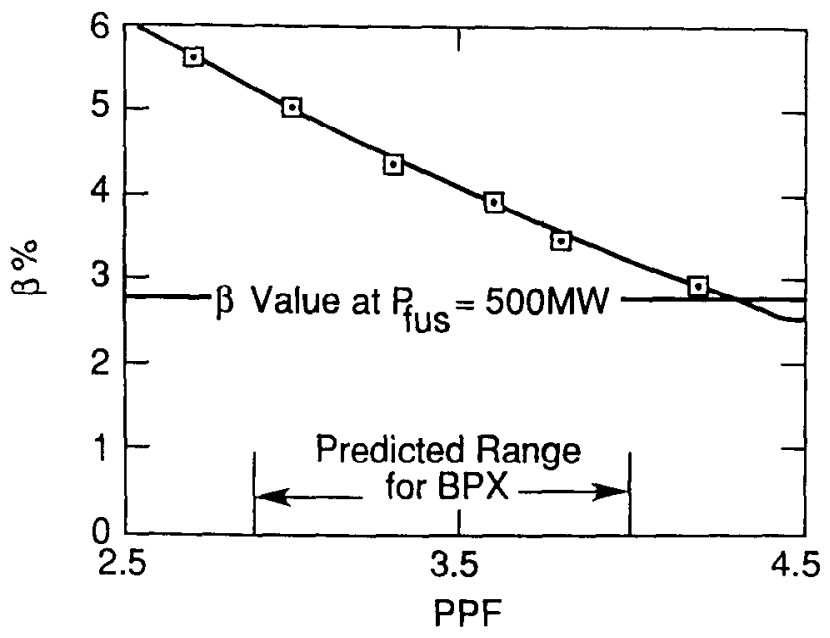

Fig. 5.71. MHD stability boundary for the current BPX parameters as a function of the pressure peaking factor $P P F$, with $R=2.59, a=0.79, \kappa=2$, $\delta=0.3$, and $1.05 \leq q \leq 3.5$.

on a limited number of surfaces. Even if this limit were locally violated, our present understanding is that this would at worst lead to enhanced local transport and not to discharge termination. ${ }^{29}$

Reducing PPF makes the profiles more favorable to ballooning stability. An optimum pressure profile was determined using the $q$ profile of the fiducial discharge, and is shown in Fig. 5.72. It is significantly broader with $P P F=2.37$ and is stable up to beta $\approx 6 \%$.

The analysis presented here has focused on the role of the pressure profile. An equally important role is played by the current profile. However, there is less freedom in varying this when considering experimentally realizable profiles. Since $q_{e d g e}$ is determined by the total plasma current and the geometry, and $q_{\text {axis }} \approx 1$, and we expect the current at the plasma edge to vanish smoothly, we have little freedom in varying the shape of the $q$ profile. Consequently, most of these studies have been done with profiles such that $0.8 \leq \ell_{i} \leq 1.0$, consistent with values inferred from experiments. ${ }^{30}$

Finally, we make a few brief remarks about the operational robustness of the plasma. Results from DIII-D on the relative frequency of survival, beta saturation or collapse, and disruption are shown in Fig. 5.73, which plots the probability of survival as a function of the Troyon coefficient $g$. It is very encouraging to note that for values of $g \leq 2$, there is a nearly $100 \%$ probability of the discharge surviving without any deleterious effects. This probability rapidly declines as $g$ approaches 3.5 ; however, since BPX is expected to operate at a value $g<2$, this result holds the promise of very safe operation.

In summary, we note that the BPX design pa- 
rameters are realistic and conservative. The stability analysis indicates a safe margin for most of the ideal MHD instabilities. The major area of uncertainty is the precise shape of the plasma profiles, which could lower the beta thresholds. However, even at the limits of the transport code predictions, marginal stability is achieved with regard to ballooning and cxternal kink modes. Stability to the internal kink mode, on the other hand, remains uncertain as it is localized near the magnetic axis, where sawtooth relaxations strongly influence the transport phenomenon and are difficult to model. Since this mode is confined to the core region, it is not normally associated with discharge termination or with degradation of confinement. Improved transport analysis for the core region with better sawtooth relaxation models should help resolve the remaining ambiguities. This is only one of the subjects of future work. Other topics include detailed analyses of specific BPX simulations, assessment of the role of resistive modes, and considerations of the interactions of kinetic effects with MHD instabilities.

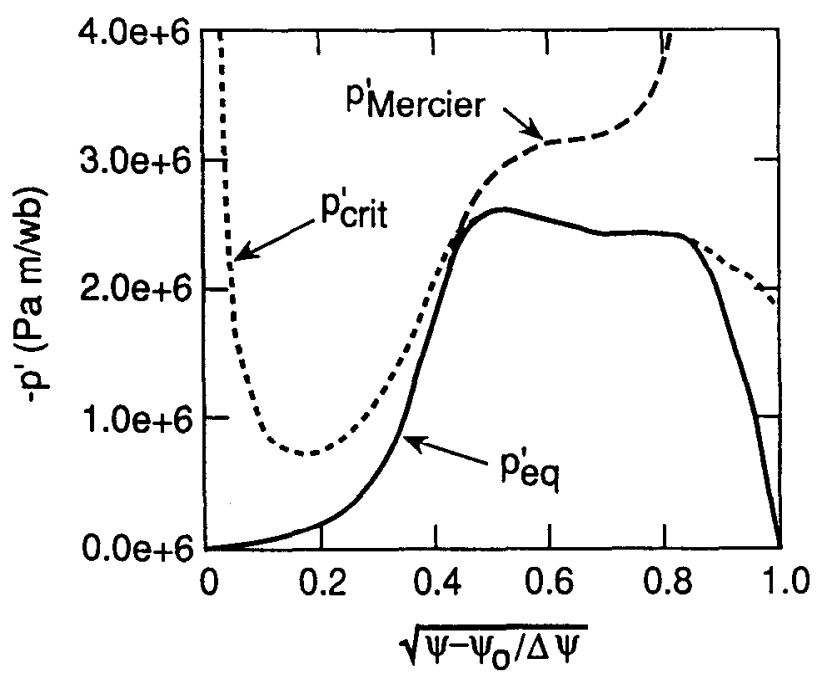

Fig. 5.72. Comparison of the optimum pressure profile for a fiducial discharge with the actual equilibrium profile.

\section{V.J. NONAXISYMMETRIC ERROR FIELD TOLERANCE}

\section{V.J.1. Error Field Effects}

Error fields are a concern for BPX because of their potential for causing nonaxisymmetric heat deposition and because of their impact on locked modes. Nonaxisymmetric heat deposition increases the heat load on the divertor plates. This problem can be exacerbated by the presence of

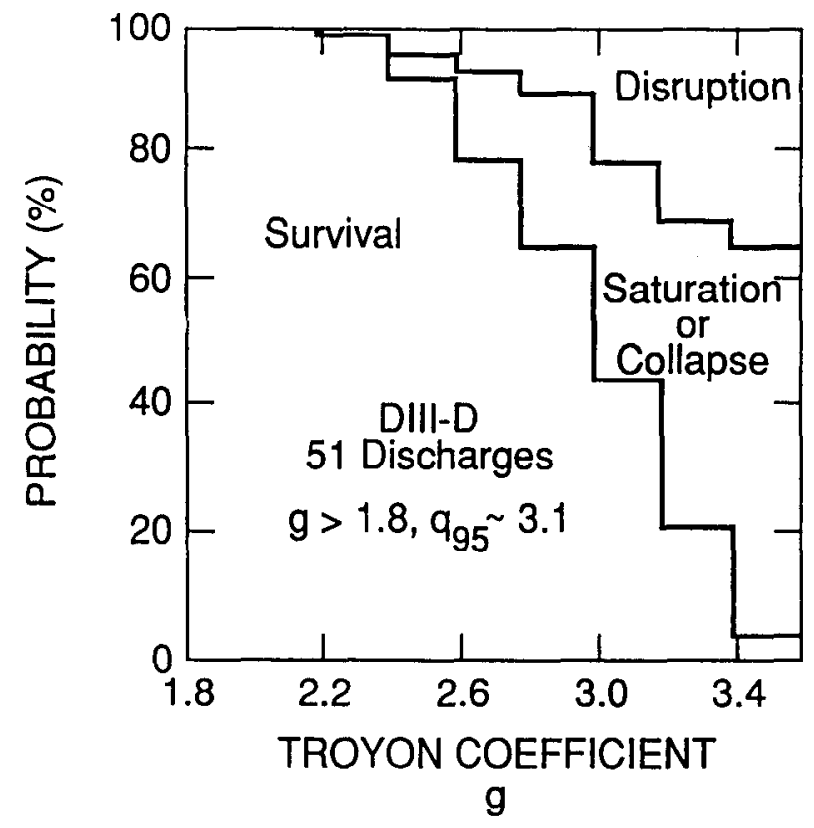

Fig. 5.73. A statistical display of 51 discharges in DIII-D with $g>1.8$ and $q_{95} \approx 3.1$, showing the probable occurrence of disruption, beta collapse, and eventless discharges, as a function of $g$.

locked modes. Locked modes on shaped crosssection tokamaks are also believed to grow to larger amplitude and to disrupt more readily than rotating tearing modes.

The toroidal variation of the energy deposition on the divertor plates has been measured on ASDEX (Ref. 31). For ICRH in the absence of locked modes, the peak heat load is found to be 10 to $20 \%$ larger than the toroidal average. This toroidal variation is believed to be caused by the presence of a $2-\mathrm{G}$ error field $\left(\delta B_{21} / B_{t} \approx 10^{-4}\right)$. For neutralbeam-heated or ohmic discharges on ASDEX, little toroidal variation is seen in the absence of locked modes. In the presence of a locked mode, neutralbeam-heated discharges show toroidal variation of up to a factor of 2 in the heat deposition. On DIII$\mathrm{D}$, the heat flux to the outer divertor strike-point bifurcates when a locked mode turns on. The data do not contain information on the toroidal dependence of the heat deposition, but it is believed that the bifurcation is associated with a toroidal variation in heat deposition. Even in the absence of a locked mode, it is found that the field in the DIII-D error compensation coil affects the heat deposition profile on the divertor. The heat deposition pattern on the bumper limiter tiles on TFTR is also found to change in the presence of a locked mode, showing a helical variation.

Locked modes are typically formed when a ro- 
tating tearing mode locks onto the external nonaxisymmetric error field and ceases to rotate. Alternatively, locked modes can also appear directly without being preceded by a rotating mode. This is the case for the low-density locked modes on DIII-D, for example. In addition to their deleterious effects on heat deposition, locked modes on shaped tokamaks are believed to be more dangerous than rotating tearing modes of the same amplitude. On JET, the growth rates of modes are often observed to increase when the mode locks. On ASDEX, active feedback of the neutral beam power has been employed to increase the momentum deposition and unlock locked modes. The motivation for this has been the belief that it reduces disruptivity.

There is also theoretical support for the idea that locked modes grow to larger amplitude than rotating modes. When a magnetic island is rotating rapidly past an error field, the growth of the island is not strongly affected by the presence of the error field. Induced wall currents can slow the mode rotation and allow the mode to lock onto the external error field. ${ }^{32}$ The error field can then strongly affect the saturation amplitude, and therefore the subsequent evolution, of the stationary island. Numerical calculations indicate that the saturation amplitude of a locked mode is sensitive to the small error field and is increased by its presence. ${ }^{33}$ The absence of wall stabilization also enhances the growth of locked modes ${ }^{34}$ over that of rotating modes.

The sensitivity of the saturation amplitude of a tearing mode to the presence of a small nonaxisymmetric perturbation is illustrated in Fig. 5.74. The figure shows the normalized radial field for a helical equilibrium solution with several different values of the error field. In the absence of the selfconsistent plasma response, the error field would decay linearly away from the boundary. For the largest error field shown, the field at the rational surface is about five times the vacuum error field and about eight times what it is in the absence of an error field.

Figures 5.75 and 5.76 show Poincaré plots for toroidal solutions with $R / a=3$ and circular cross section, without and with an error field. (We caution that the origin of our coordinate system does not lie on the magnetic axis, so the region about the axis looks like an island but is not.) The tearing mode is unstable. In the absence of an error field, there are saturated islands at the $q=2$ and $q=3$ rational surfaces, with the total island width across the midplane about $6 \%$. The solution in Fig. 5.76 has an error field imposed with resonant $n=1$ and $n=2$ Fourier components. The magnitudes of the Fourier components were calculated from a small shift and elliptical deformation of a nearly

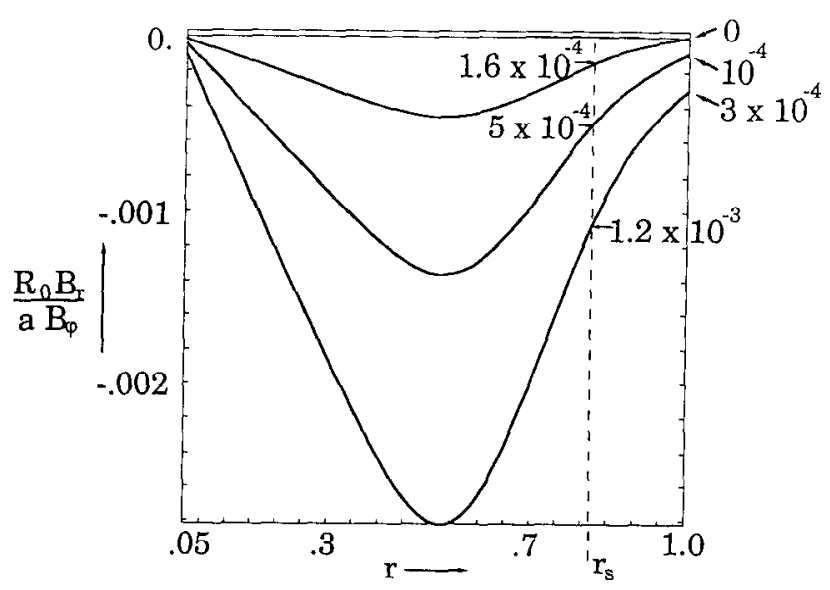

Fig. 5.74. Normalized radial field for a helical equilibrium solution with several different values of the error field.

circular PF coil, with the coil current and shift chosen to approximately reproduce the reported $n=1$ error field spectrum on DIII-D (Ref. 35). The 2/1 Fourier component has an amplitude of $5.5 \times 10^{-5} B_{t}$ at the boundary. Other components decrease in amplitude with increasing $m$. With the imposed error field, the islands are larger and more numerous. The total island width across the midplane is now about $23 \%$.

Theoretical calculations also show large effects for stable current profiles just below the instability threshold, due to the strong amplification of error fields. ${ }^{36}$ In the absence of an error field, the island width is zero at the threshold. In the presence of an error field, a large island may be produced in the plasma. The presence of an error field broadens the range of plasma parameters over which deleterious tearing effects are seen.

The observed behavior of low-density locked modes on DIII-D (Ref. 35) is consistent with these theoretical results. The density threshold has been found to depend systematically on the field in the error compensation coil. The results are independent of $q$. In particular, the lowest densities are attained with a current of about $1.5 \mathrm{kA}$ in the error compensation coil. With this current, the field from the coil is believed to be partially canceling the error fields present in DIII-D. Although systematic studies have been done only for the lowdensity locked modes, locked modes occur on DIII$\mathrm{D}$ in all parameter regimes, and the error compensation coil is believed to improve the behavior of the device in all regimes.

Small nonaxisymmetric fields have also been found to affect the threshold power for the L$\mathrm{H}$ transition in experiments on JFT2-M with a poloidal divertor plus ergodic magnetic limiter 


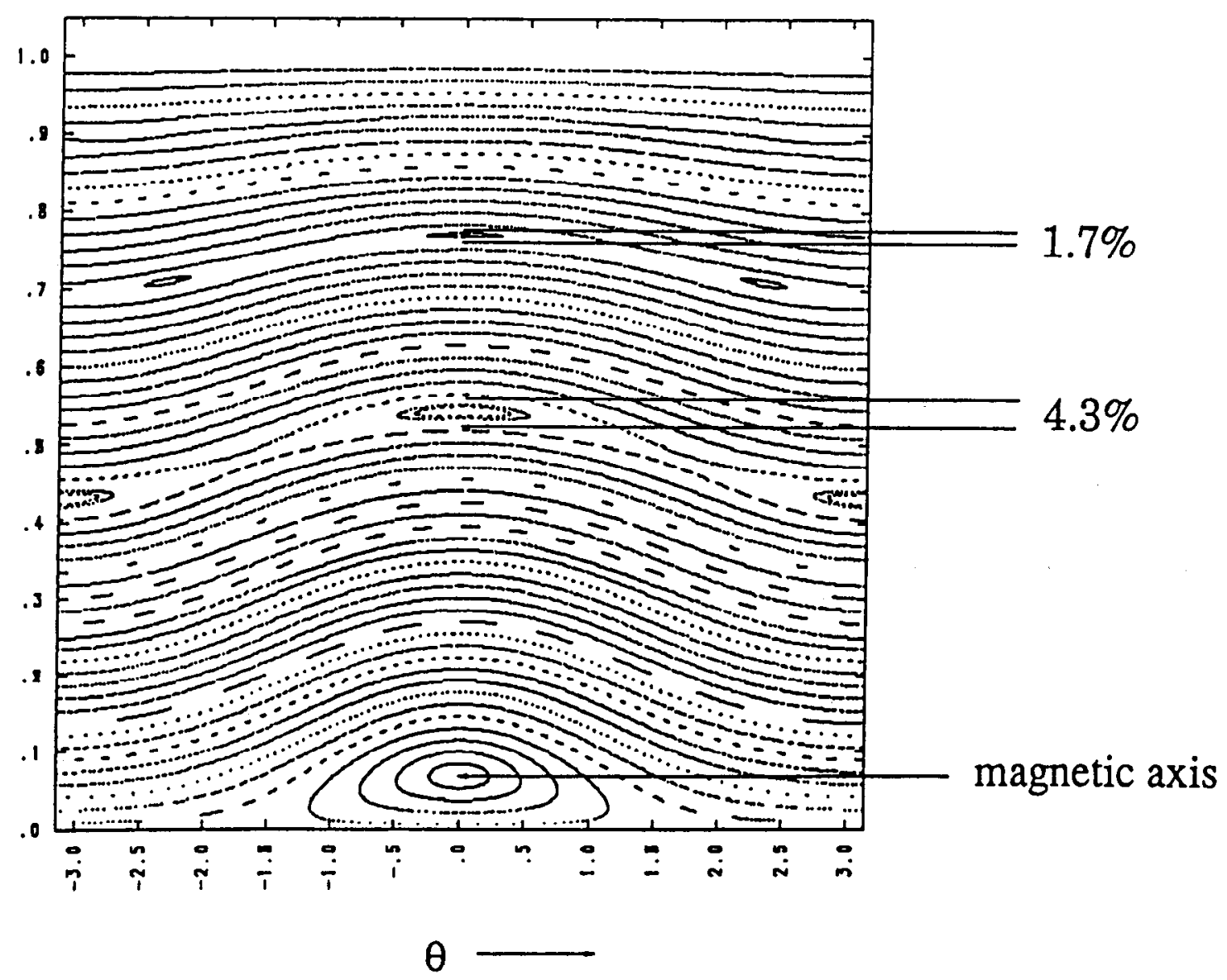

Fig. 5.75. Poincaré plot for a toroidal solution with $R / a=3, \gamma=1$, and no error field. 
Jardin et al. MHD EQUILIBRIUM AND STABILITY

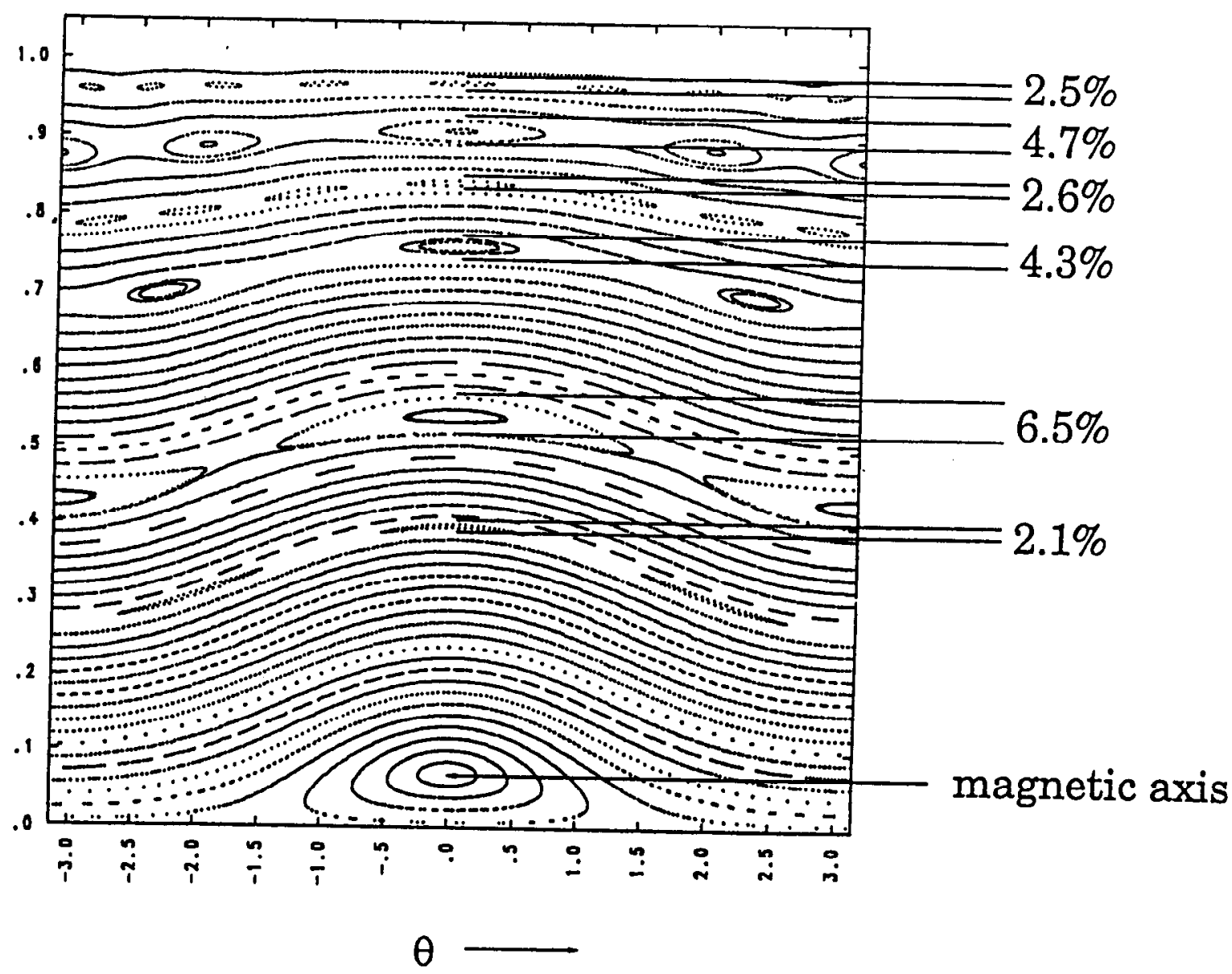

Fig. 5.76. Poincaré plot for a toroidal solution with $R / a=3, \gamma=1$, and error field as described in the text. 
coils. ${ }^{37}$ Running with a range of currents in the ergodic magnetic limiter, it was found that the $\mathrm{H}$ mode power threshold increased from $350 \mathrm{~kW}$ at zero current to about $600 \mathrm{~kW}$ for $\delta B_{n m} / B \approx 2$ to $5 \times 10^{-4}$.

Locked modes decrease energy confinement by decreasing the effective plasma aperture. ${ }^{38}$ In the absence of locked modes, error fields are not observed to cause large effects on confinement. In the EML experiments on JFT-2M, an $\mathrm{H}$ mode is obtained for the largest limiter currents, even though it is believed that there is a large ergodic region at the outside of the plasma. No effect on global confinement was observed for the H-mode discharges. The edge temperature and density were reduced by about 5 to $10 \%$. In a series of L-mode discharges in DIII-D that were identical except that the current in the error compensation coil was varied from $-3000 \mathrm{~A}$ to $4000 \mathrm{~A}$, the change in the stored energy was less than $2 \%$, and the change in the temperature was less than $3 \%$.

\section{V.K. BPX ERROR FIELD TOLERANCE}

We set a field error tolerance for BPX that allows us to avoid or minimize the deleterious effects described above. To obtain some guidance, we first look at puncture plots produced by field line tracing with vacuum error fields. Figures 5.77 and 5.78 show the result of field line tracing for the $R=2.14 \mathrm{~m} a=0.65 \mathrm{~m} \mathrm{BPX}$ without and with error fields. The error fields for Fig. 5.78 are the vacuum fields produced by coil PF1 only at 18.8 MA, shifted $3 \mathrm{~mm}$ and given an ellipticity of 1.0103. The calculation does not take into account the plasma response to the external perturbation. The $2 / 1$ island is about $3 \mathrm{~cm}$ wide at the toroidal angle shown but only about $0.9 \mathrm{~cm}$ wide where the O-point is on the outboard midplane. There is also an island of comparable width at the $q=3$ surface.

To determine how the island widths will scale as the strength of the error field is varied, we use an analytical expression valid in a cylinder with narrow islands. In that case, the island width is given by ${ }^{39}$

$$
w \approx 4\left(\frac{R B_{r m n} q^{2}}{m B_{z} q^{\prime}}\right)^{1 / 2},
$$

where $B_{r n m}$ is the resonant Fourier component of the radial field at the rational surface. This expression roughly reproduces the $m=2, n=1$ island width of Fig. 5.73 if we substitute a reasonable estimate for $B_{r n m}$ at the rational surface (the quoted $B_{r n m}$ is on a circular reference surface at

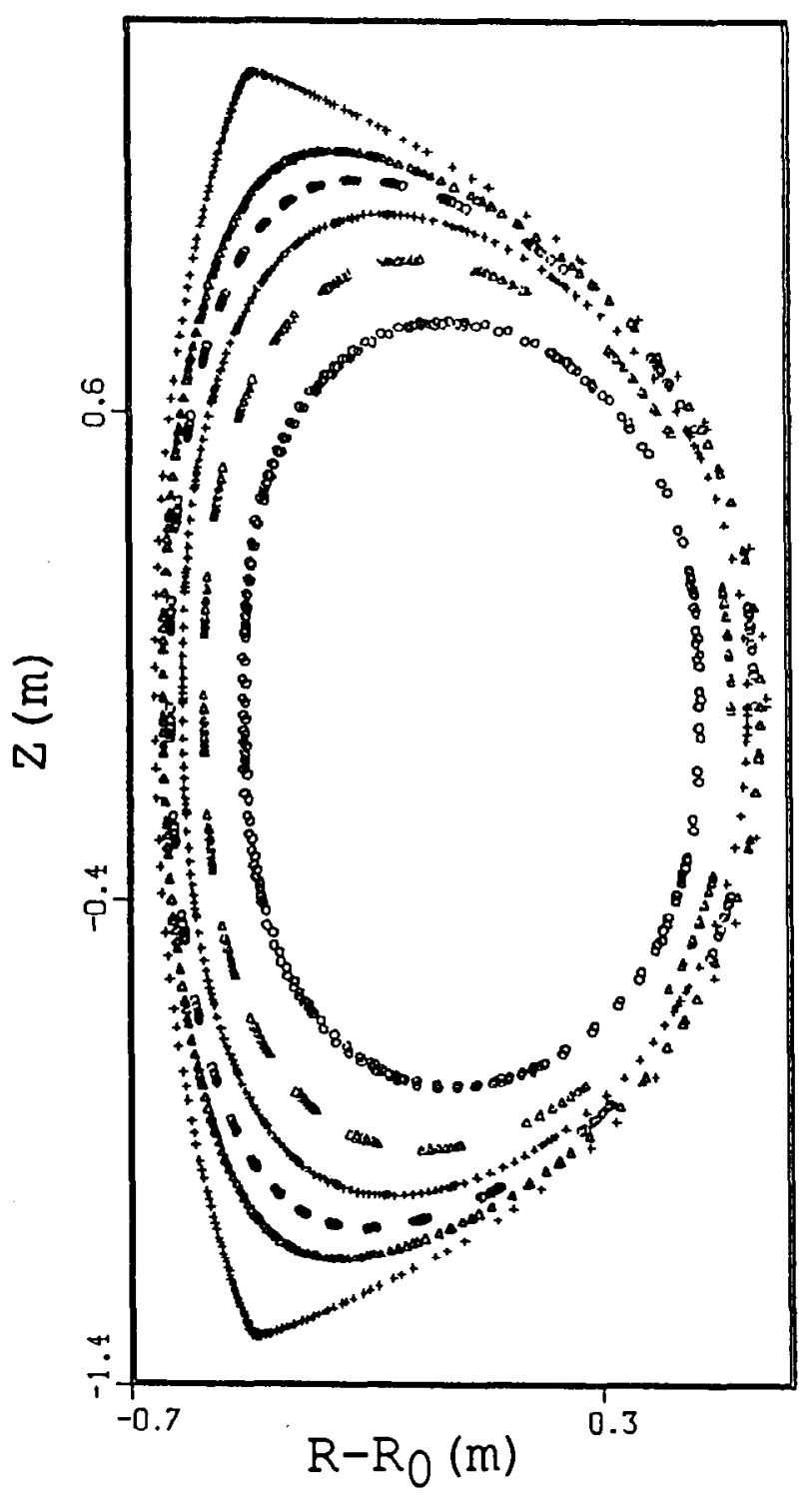

Fig. 5.77. Field line tracing puncture plot for the unperturbed BPX $R=2.14 \mathrm{~m} a=0.65 \mathrm{~m}$ configuration. Field lines are traced for $q=3 / 2,2 / 1,5 / 2$, and $3 / 1$ and at the separatrix.

$r=0.65 \mathrm{~m})$ and if we put in a reasonable estimate for $q^{\prime}$. When the error fields for the field line tracing are decreased by an order of magnitude, the island widths obey the predicted scaling with $\sqrt{B_{r n m}}$.

Equation (5.15) suggests that the total fraction of the plasma cross section occupied by islands is proportional to

$$
\sum \frac{1}{n}\left(\frac{m B_{r m n}}{B}\right)^{1 / 2}
$$

We have taken $q=m / n$ and have treated $q^{\prime} a$ as a constant independent of $q$ to obtain this expression. Only the Fourier components that resonate 


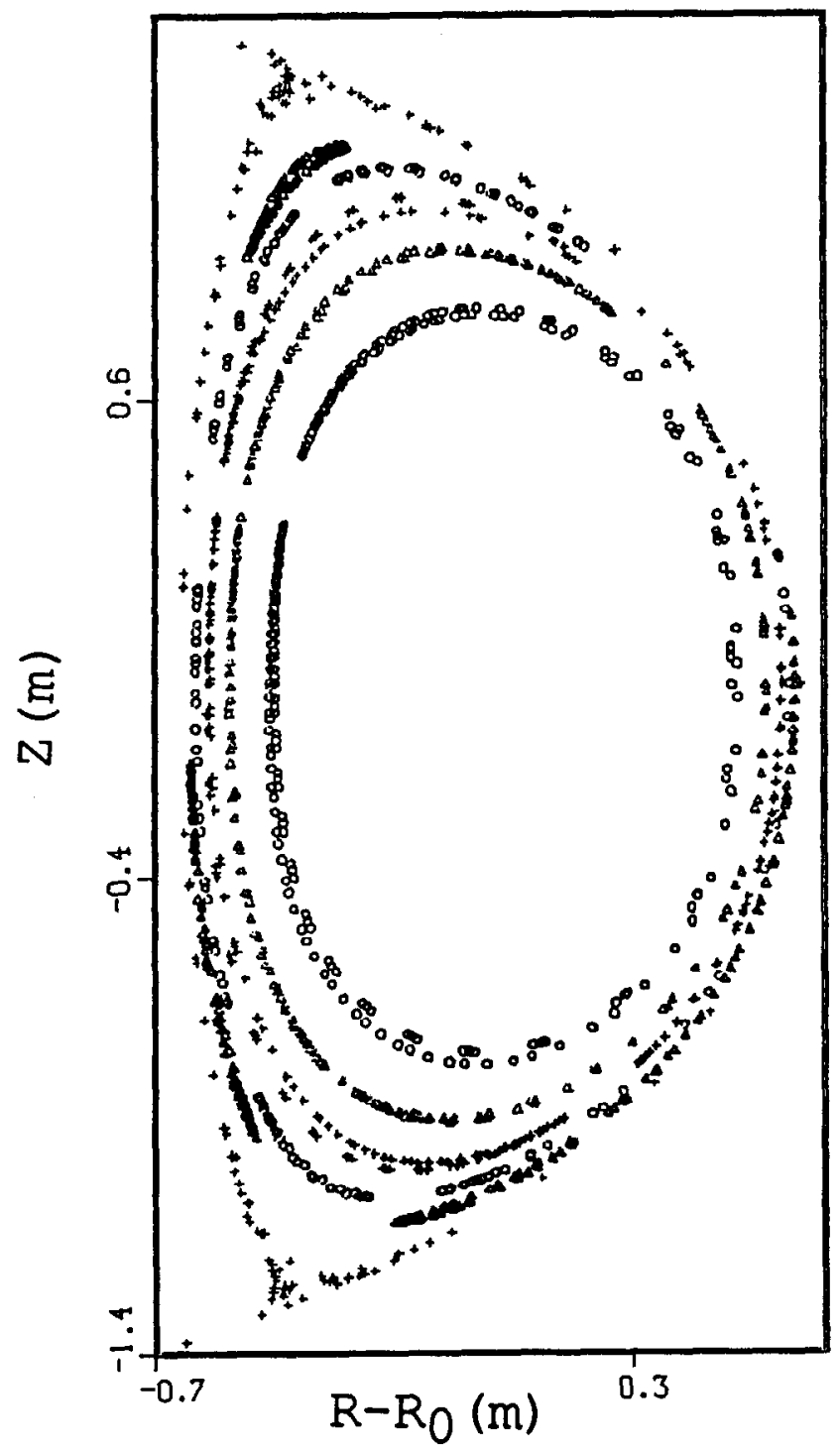

Fig. 5.78. Puncture plot with field errors from PF 1 only at $-18.8 \mathrm{MA}$-turns shifted by $3 \mathrm{~mm}$ and with ellipticity 1.0103 . The vacuum error field is used here. (No self-consistent plasma response.)

with rational surfaces in the plasma should be retained in the sum. We therefore restrict the sum to $1 \leq m / n \leq 5$.

Equation (5.16) is expressed in terms of the field at the rational surface. For convenience in evaluation, we prefer to express our criterion in terms of the Fourier components on a circular reference surface. The vacuum Fourier components go like $r^{m-1}$. For a conservative field error criterion, it is reasonable to use the Fourier components on the reference surface directly in the above expression.

We make use of the results from field line following to normalize our expression for the field error tolerance. We adopt a field error tolerance of the form

$$
\sum_{1 \leq m / n \leq 5} \frac{1}{n}\left(\frac{m B_{r m n}}{B}\right)^{1 / 2}<E .
$$

Evaluating Eq. (5.17) for the case where PF1 at $19 \mathrm{MA}$ has a 3.6-mm shift and ellipticity, we find that the sum is 0.09 . Figure 5.78 shows that the vacuum islands occupy an acceptable $6 \%$ of the plasma cross section in this case. To determine an acceptable value for $E$, we must take into account the self-consistent plasma response, which has not been included in Fig. 5.78.

The numerical calculations give us an indication of how much amplification to expect from the selfconsistent plasma response. The error field spectrum corresponding to Fig. 5.79 has $E=0.074$. This calculation was done for a circular cross section, and we can expect the islands to be somewhat larger in a shaped cross section. For the circular cross section shown, we find that a further increase in the amplitude of the error field gives a rapid increase in the total island width due to nonlinear coupling of the islands to higher harmonics. This suggests that the error field amplitude cannot be increased much further without causing a plasma disruption. These results suggest that we take $E \approx 0.05$. This value is also consistent with cylindrical calculations, such as those shown in Fig. 5.69, which indicate an amplification of the error field by up to an order of magnitude by the plasma currents.

Fourier analysis of field error measurements on DIII-D also yields similar conclusions. Figure 5.79 shows the results of such an analysis. ${ }^{40}$ The corresponding value of $E$ is about 0.1. At this level of the error field, significant deleterious error field effects are seen. Improvements in performance are seen down to the lowest levels of the error field achievable with the error compensation coil. At these levels, the 2/1 Fourier harmonic is reduced by a factor of $\sim 4$, and other harmonics are reduced somewhat less. Based on these results, a value of $E=0.05$ in the error field tolerance is again reasonable.

The error field tolerance we have arrived at should constrain the heat load nonaxisymmetry to an acceptable level. As discussed above, the 10 to $15 \%$ toroidal heat load asymmetry in ASDEX is believed to arise from a $\delta B_{21} / B_{t} \approx 10^{-4}$. This suggests that the error field magnitude in ASDEX is comparable to that in DIII-D and that the constraint implied by $E=0.05$ will maintain the heat load asymmetry at an acceptable level. Similarly, the constraint will maintain the error field magnitude below the level at which effects on the $\mathrm{L}$ - 


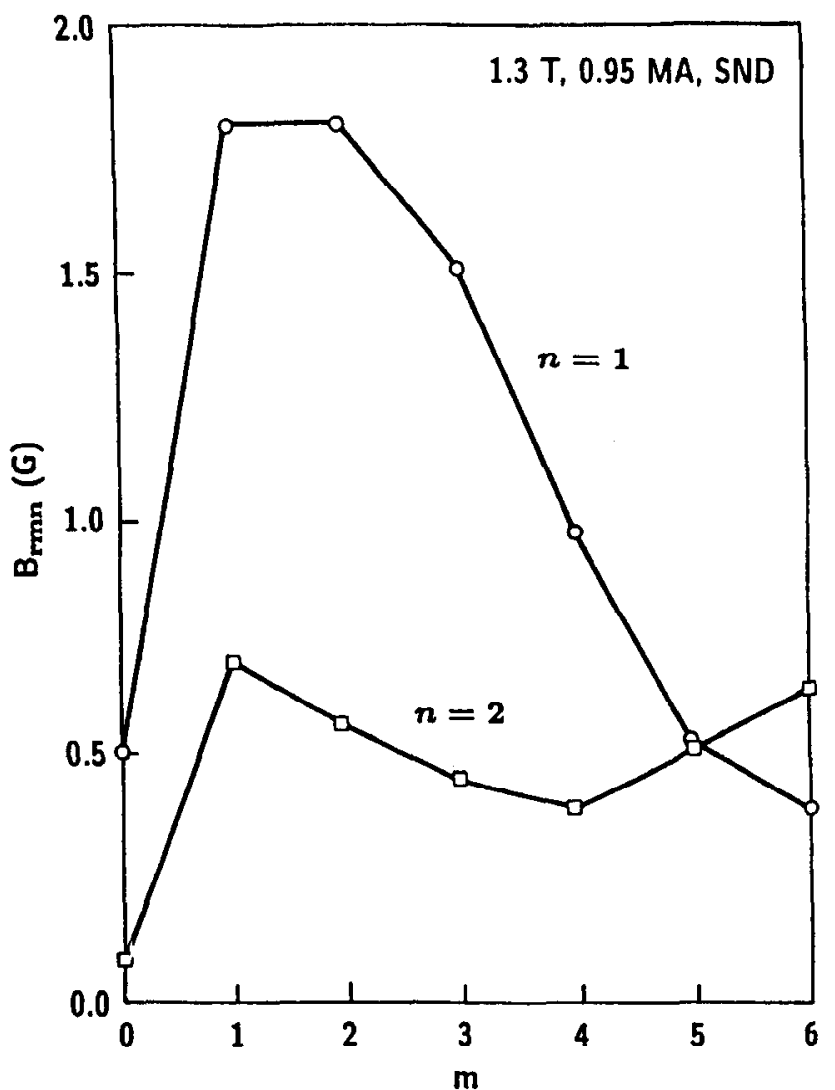

Fig. 5.79. Fourier analysis of the helical, radial error fields of the sum of all field coils for discharge \#65489 on DIII-D. The analysis is on a torus of minor radius $0.68 \mathrm{~m}$, major radius $1.67 \mathrm{~m}$.

$\mathrm{H}$ transition were observed in the JFT2-M experiments.

To give a feeling for the implied construction tolerances, Fig. 5.80 shows the error field spectrum produced by a 3-mm shift of coil PF7. Substituting these Fourier amplitudes into the left-hand side of Eq. (5.17) gives a value of 0.022 .

\section{V.L. TOROIDAL FIELD RIPPLE}

The toroidal field strength in BPX varies periodically in strength as one proceeds toroidally around the device due to the discrete nature of the TF coils. Because there are $N=18$ coils, the dominant variation in toroidal field strength is in the $N$ 'th harmonic. The toroidal field strength is thus well represented by the parameterization

$$
B(R, \phi, Z)=B_{o}(R, Z)[1+\delta(R, Z) \sin (N \phi)],
$$

where $(R, \phi, Z)$ are standard cylindrical coordinates. We refer to $\delta(R, Z)$ as the TF field ripple. It is sometimes quoted in percent.

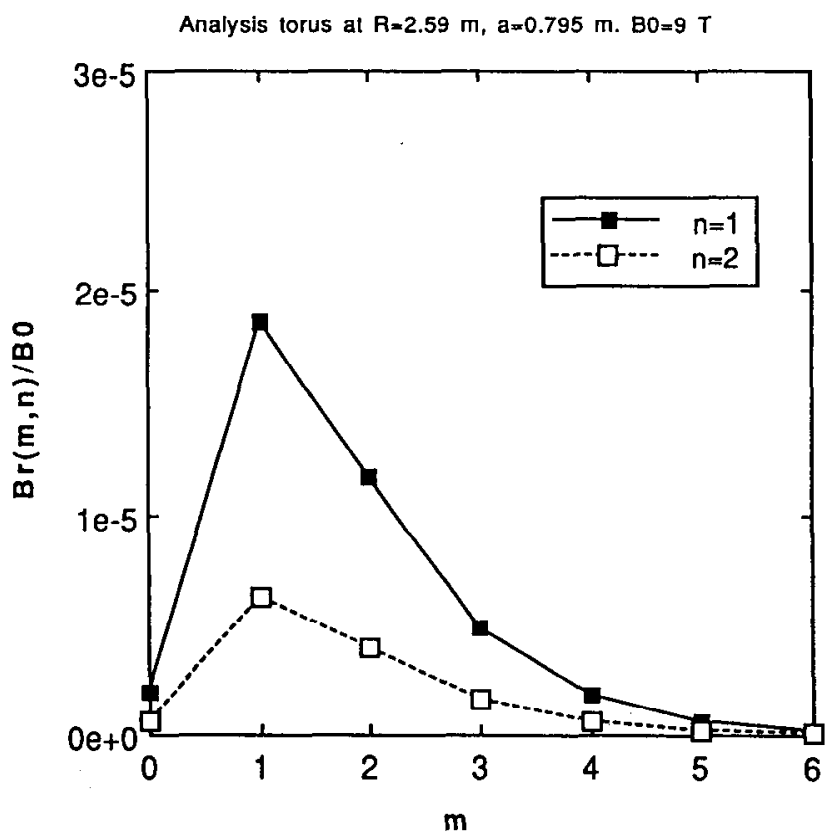

Fig. 5.80. Normalized BPX error field harmonics for PF7 at $I=4.8 \mathrm{MA}$ with 3-mm shift.

Figure 5.81a shows contours of the TF ripple $\delta(R, Z)$ in BPX, superimposed upon an EOB equilibrium plasma for which three flux surfaces are shown. The contours in the figure correspond to $0.01,0.02,0.05,0.1,0.2$, and $0.5 \%$. They were calculated using the Biot-Savart law and the actual TF coil geometry. To a good approximation, the field ripple on the horizontal midplane can be approximated by

$$
\delta(R, Z=0)=100\left(R / R_{0}\right)^{N_{\%}},
$$

where $R_{0}=4.47$.

The primary concern regarding $\mathrm{TF}$ ripple in BPX is that it leads to enhanced alpha-particle losses. Numerical simulation and analytical theory have shown that the dominant mechanism affecting this ripple-induced loss is that of stochastic diffusion of the tips of the orbits of the toroidally trapped alpha particles. ${ }^{41-44}$

To a good approximation, this loss mechanism can be predicted by the extent to which the TF ripple at the trapped alpha particle turning point exceeds the critical ripple $\delta_{\text {crit }}$ for loss on that magnetic flux surface. Thus, if $\delta_{A}$ is the maximum actual field ripple amplitude on a given flux surface, then some fraction of the particles born on that surface will be lost for

$$
\delta_{A}>\delta_{\text {crit }}
$$



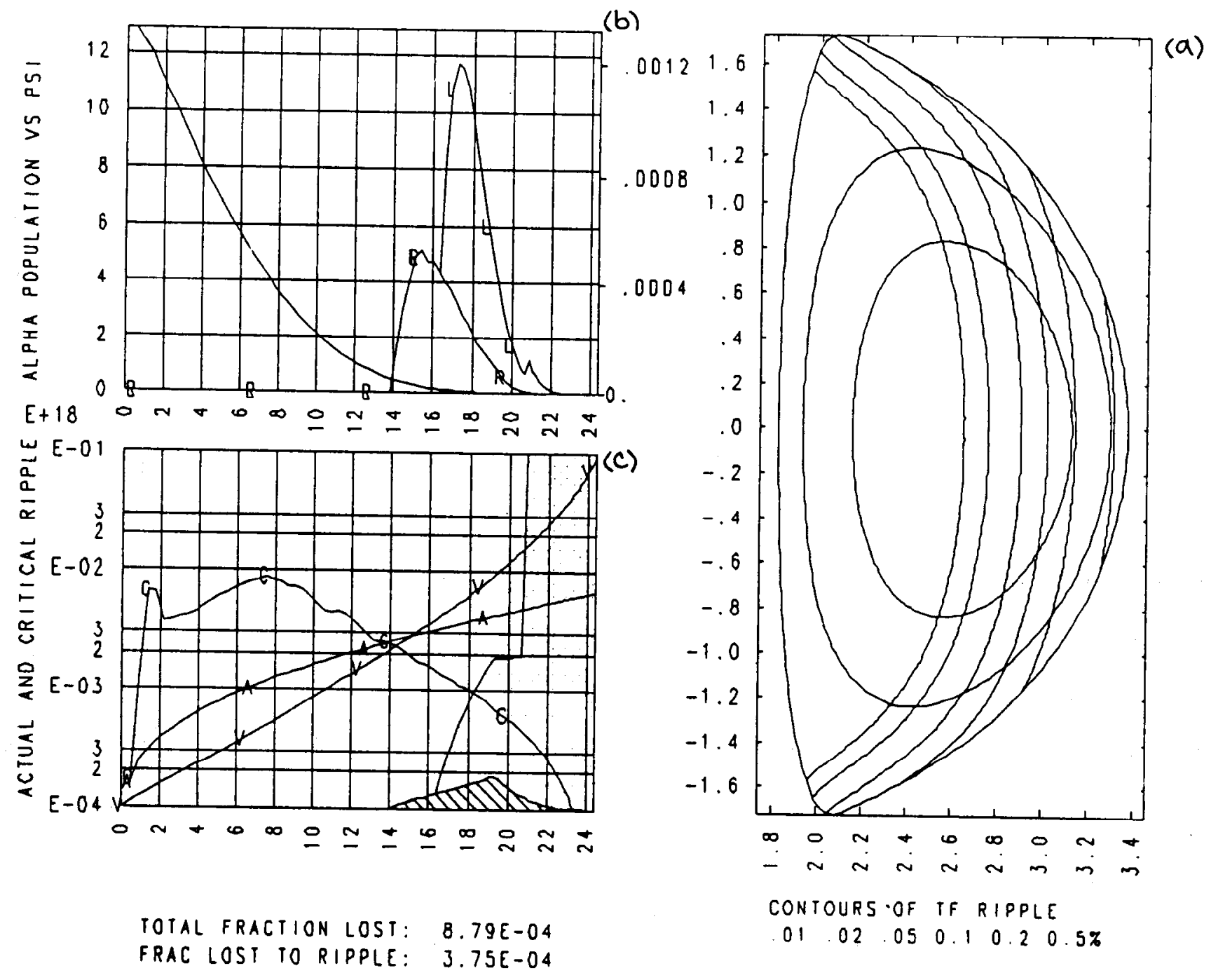

Fig. 5.81. (a) Contours of $0.01,0.02,0.05,0.1,0.2$, and $0.5 \%$ ripple (b) alpha-particle distributions (left) and relative losses (right), and (c) critical and actual ripple (left) and percentages due to ripple (hatched) 
where

$$
\delta_{c r i t}=\frac{\left(\frac{1}{B} \frac{d B}{d \phi}\right)_{b}^{1 / 2}}{N \frac{d \phi_{b}}{d \psi_{p}} \sqrt{\pi N} g \rho}
$$

Here $\psi_{p}$ is the poloidal flux per radian, $g=R B_{T}, \rho$ is the gyroradius, and $(d B / d \phi)_{b}$ indicates the total derivative of the axisymmetric $B$ along a field line, evaluated at the bounce point. Equations (5.20) and (5.21) are those evaluated by the RIPLOS program, which has been incorporated as a subroutine in the TSC code. We also calculate the orbit loss, which does not involve field ripple, but just occurs when the alpha-particle orbit hits the limiter and is lost.

Figure $5.81 \mathrm{~b}$ has three curves on it. The bottom scale in this and in Fig. 5.81c is the plasma poloidal flux, with the magnetic axis being on the left and the plasma edge being on the right. The unmarked curve in Fig. 5.81c is the alpha-particle distribution at EOB as calculated in the TSC fiducial discharge. It is seen to be strongly peaked in the center, reading a central value of about $n_{0 \alpha}=1.3 \times 10^{19} \mathrm{~m}^{-3}$, as indicated by the scale on the left. The curves marked " $L$ " and " $R$ " refer to the scale on the right, which is in units such that $n_{0 \alpha}=1$. This indicates the number density of alpha particles lost due to orbit losses and ripple losses (the $L$ curve) and due to ripple losses only (the $R$ curve). This is seen to be a small effect, impacting only those small numbers of alpha particles born on the outer surfaces.

Figure 5.81c has three curves and two shaded regions. The curves marked " $C$ " and " $A$ " refer to the scale on the left. They are the maximum values of the critical ripple (C) and the actual ripple (A) for each flux surface as defined in Eq. (5.20). The curve marked "V" is on a linear scale, merely indicating the relative volume contained within each flux surface.

The dotted region and cross-hatched regions are also on linear scales (not shown) going from 0 to $100 \%$. The dotted region refers to what fraction of the alpha particles born on that surface are lost due to both orbit loss and ripple loss, and the crosshatched region indicates the percentage lost due to ripple loss only. By comparing these percentages with the curves in Fig. $5.81 \mathrm{~b}$, we see that these percentages are non-negligible only in the outer regions of the plasma where very few alpha particles are produced.

Finally, in summing both orbit loss and ripple loss effects over the entire plasma, we find that only $0.087 \%$ of the alpha particles are lost, and only $0.037 \%$ are lost due to field ripple. Decreasing the field ripple in the machine further would not have much effect, since the alpha-particle loss associated with the field ripple is already smaller than the orbit loss, which would not decrease with decreasing field ripple.

\section{REFERENCES}

1. R. PILLSBURY, "PF Coil Positions and Current Waveforms for an 11.8 MA, $9 \mathrm{~T}, 100 \mathrm{MW}$ Diverted Discharge in a $2.59 \mathrm{~m}$ Wedge Design for BPX," GEM-47, Massachusetts Institute of Technology (1991).

2. S. C. JARDIN, N. POMPHREY, and J. DELUCIA, "Dynamic Modeling of Transport and Positional Control of Tokamaks," J. Comput. Phys., 66, 481 (1986).

3. "Compact Ignition Tokamak General Requirements Document," W-901012-PPL-XX, Princeton Plasma Physics Laboratory (Oct. 1990).

4. D. J. STRICKLER, Y-K. M. PENG, N. POMPHREY, and S. C. JARDIN, "Equilibrium Calculations for Plasma Control in BPX," ORNL/FEDC-89/.4, Oak Ridge National Laboratory (Jan. 1990).

5. D. J. STRICKLER, Y-K. M. PENG, S. C. JARDIN, and N. POMPHREY, "MHD Equilibrium Using Free-Boundary Control Matrix," Bull. Am. Phys. Soc., 34, 1972 (1989).

6. D. J. STRICKLER, Y-K. M. PENG, S. C. JARDIN, and N. POMPHREY, "Dependence of BPX PF Coil Currents on Profile and Shape Parameters Using the Control Matrix," Fusion Technol., 19, 1452 (1991).

7. R. D. PILLSBURY, "PF Coil Positions and Current Waveforms for an 11.8 MA, 9T, Diverted Discharge in a $2.59 \mathrm{~m}$ Wedged Design for BPX," GEM-46, Plasma Fusion Center, Massachusetts Institute of Technology (Aug. 1990).

8. F. HOFMANN, N. POMPHREY, and S. C. JARDIN, "Application of a New Algorithm to Plasma Shape Control in BPX," submitted to $\mathrm{Nu}$ clear Fusion (1991).

9. F. HOFMANN and S. C. JARDIN, "Plasma Shape and Position Control in Highly Elongated Plasmas," Nucl. Fusion, 30, 2013 (1990).

10. F. HOFMANN and G. TONETTI, "Fast Identification of Plasma Boundary and X-points in Elongated Tokamaks," Nucl. Fusion, 28, 519 (1988).

11. D. J. WARD, PhD Thesis, Princeton University (1990).

12. S. HANEY, "Methods for the Design and Optimization of Shaped Tokamaks," PhD Thesis, MIT (1988). 
13. W. M. TANG, "Microinstability-Based Model for Anomalous Thermal Confinement in Tokamaks," Nucl. Fusion, 26, 1605 (1986); see also S. C. JARDIN, J. DELUCIA, M. OKABAYASHI, N. POMPHREY, M. REUSCH, S. KAYE, and H. TAKAHASHI, "Modelling of Post-Disruptive Plasma Loss in the Princeton Beta Experiment," Nucl. Fusion, 27, 569 (1987).

14. S. C. JARDIN, M. G. BELL, and N. POMPHREY, "TSC Simulation of Ohmic Discharges in TFTR," Bull. Am. Phys. Soc., 35, 1921 (1990).

15. M. ZARNSTORFF et al., "Parallel Electric Resistivity in the TFTR Tokamak," Phys. Fluids. $B, 2,1852$ (1990); see also Princeton Plasma Physics Laboratory report PPPL-2675 (1990).

16. S. KAYE, BPX Interdepartmental Memo (Jan. 28, 1991).

17. A. VANNUCCI and R. D. GILL, "JET High Current Disruptive Pulses $\left(\mathrm{I}_{p}^{\max } \geq 5 \mathrm{MA}\right)$," JETIR (89) 15.

18. C. Z. CHENG, H. P. FURTH, and A. H. BOOZER, "MHD Stable Regime of the Tokamak," Plasma Phys. Controlled Fusion, 29, 351 (1987).

19. D. W. WEISSENBURGER and U. R. CHRISTENSEN, "A Network Mesh Method to Calculate Eddy Currents on Conducting Surfaces," IEEE Trans. Magnetics, MAG-18, 422 (1982); see also D. W. WEISSENBURGER, "SPARK Version 1.1 User Manual," PPPL-2494 Princeton Plasma Physics Laboratory (1988).

20. R. O. SAYER, Y-K. M. PENG, and S. C. JARDIN, "Disruption-Induced Forces on a Vacuum Vessel from TSC Poloidal Eddy Currents," Bull. Am. Phys. Soc., 33, 1972 (1988).

21. H. W. KUGEL et al., "Induced Voltage and Eddy Current in the PBX-M Stabilizing Shell," Proc. 16th European Conf. Controlled Fusion and Plasma Physics, Venice, Italy, March 13-17, 1989.

22. A. G. KELLMAN et al., "Vertical Stability, High-Elongation, and the Consequences of Loss of Vertical Control on DIII-D," Proc. 16th Symp. Fusion Technology, London, UK, September 3-7, 1990, GA-A20238 (1990).

23. R. O. SAYER, Y-K. M. PENG, and S. C. JARDIN, "TSC Halo Disruption-Induced Forces on the BPX Vacuum Vessel," Bull. Am. Phys. Soc., 35, 1921 (1990).

24. R. O. SAYER, "TSC Disruption Scenarios and BPX Vacuum Vessel Force Evolution," ORNL/FEDC-89/3, Oak Ridge National Laboratory $(1990)$.
25. D. J. STRICKLER, J. B. MILLER, K. E. ROTHE, and Y-K. M. PENG, "Equilibrium Modeling of the TFCX Poloidal Field Coil System," ORNL/FEDC-83/10, Oak Ridge National Laboratory (Apr. 1984).

26. F. TROYON et al., "MHD-Limits to Plasma Confinement," Plasma Phys. Controlled Fusion, 26, 209 (1984)

27. J. JOHNSON et al., "MHD Stability of the 11 MA TSC Fiducial Discharge and the Effect of Varying Plasma Parameters and Shapes About It" AE-890804-PPL-08 Princeton Plasma Physics Laboratory (1989).

28. M. PHILLIPS et al., "MHD Stability Properties of a High Current, High Beta Tokamak," Nucl. Fusion, 28, 1499 (1988).

29. T. OZEKI et al., "Ideal MHD Stability of Pellet Fuelled Plasmas in JT-60," Nucl. Fusion, 31, 51 (1991)

30. A. KELLMAN, GA, Private Communication (1990).

31. T. E. EVANS, J. NEUHAUSER, F. LEUTERER, E. R. MUELLER, and the ASDEX Team, "Characteristics of Toroidal Energy Deposition Asymmetries in ASDEX," Technical Report GA-A20076, General Atomics (1990).

32. M. F. F. NAVE and J. A. WESSON, "Mode Locking in Tokamaks," Proc. 14th European Conf. Controlled Fusion and Plasma Physics Madrid, Spain, June 22-26, 1987, Vol. 11D, p. 1103, European Physical Society (1987).

33. A. REIMAN and D. MONTICELLO, "Sensitivity of Tokamak Equilibria to the Presence of Nonaxisymmetric Error Fields - Numerical Computations," to be published.

34. M. PERSSON and A. BONDESON, "Wall Locking and Density Limit Disruptions," Nucl. Fusion, 29, 989 (1989).

35. T. SCOVILLE et al., "Prevention of the $n=1$ Locked Mode in DIII-D Plasmas," Bull. Am. Phys. Soc., 34, 2118 (1989).

36. A. REIMAN, "Tokamak Magnetic Islands in the Presence of Nonaxisymmetric Perturbations," to be published.

37. A. W. LEONARD et al., "Ergodic Magnetic Limiter Experiments on JFT-2M," Bull. Am. Phys. Soc., 35, 1949 (1990).

38. J. A. SNIPES, D. J. CAMPBELL, T. C. HENDER, M. V. HELLERMANN, and H. WEISEN, "Plasma Stored Energy and Momentum Losses During Large MHD Activity in JET," Nucl. Fusion, 30, 205 (1990).

FUSION TECHNOLOGY VOL. $21 \quad$ MAY 1992 
39. J. WESSON, "Tokamaks," Oxford Engineering Science Series, Vol. 20, p. 169.

40. R. J. LaHAYE and J. T. SCOVILLE, "A Method to Measure Poloidal Field Irregularities in Toroidal Plasma Devices," Rev. Sci. Inst., 62, 2146 (1991).

41. R. WHITE, Theory of Tokamak Plasmas, North-Holland (1989).
42. A. H. BOOZER, "Enhanced Transport in Tokamaks due to Toroidal Ripple," Phys. Fluids, 23, 2283 (1980).

43. R. GOLDSTON and H. TOWNER, "Effects of Toroidal Field Ripple on Suprathermal Ions in Tokamak Plasmas," J. Plasma Phys., 26, 283 (1981).

44. P. N. YUSHMANOV, "Generalized RippleBanana Transport in a Tokamak," Nucl. Fusion, 23, 1599 (1983). 